\title{
DXPDVIMENTAL
}

\section{BIOLOGY MONOGRAPHS}

\subsection{3 \\ D 48}




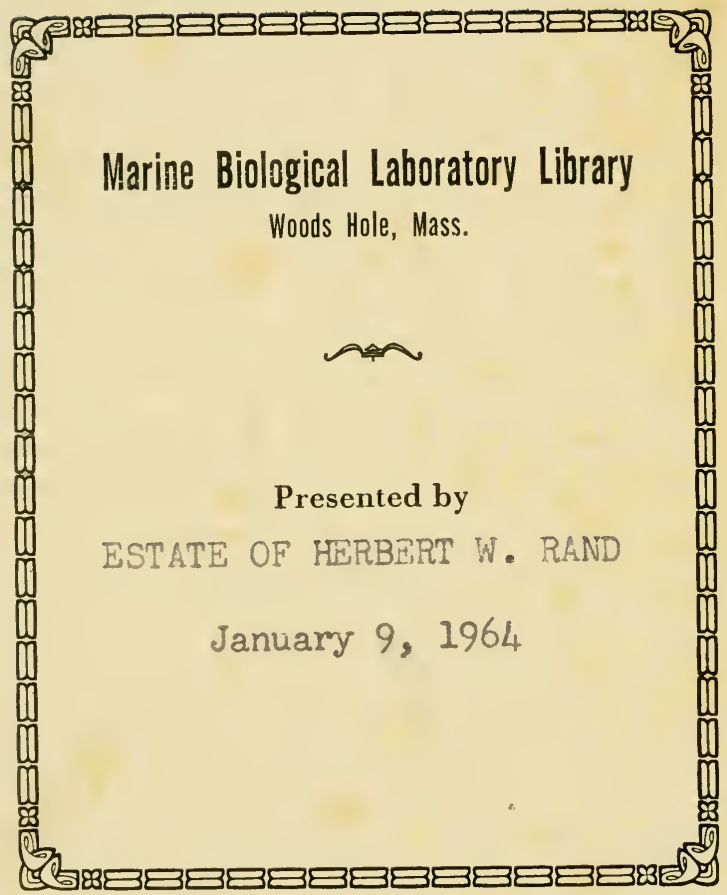




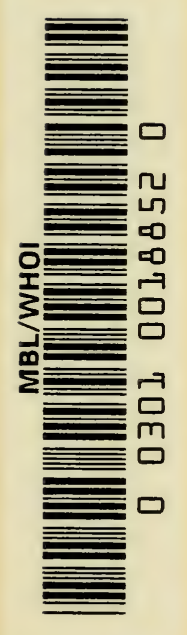


VERTEBRATE PHOTORECEPTORS 
EXPERIMENTAL BIOLOGY MONOGRAPHS

Editors: PhiliP BARD, Johns Hopkins University; L. R. Blinks, Stanford University; W. B. CANNON, Harvard University; W. J. Crozier, Harvard University; J. B. Collip, McGill University; Hallowell Davis, Harvard University; S. R. Detwiler, Columbia University; Selig Hecht, Columbia University; Hudson Hoagland, Clark University; J. H. NoнтнRоP, Rockefeller Institute for Medical Rescarch; G. II. PARKer, Harvard University; Gregory Pincus, Clark University; L. J. StADLER, The University of Missouri; Sewall Wright, University of Chicago.

PACEMAKERS IN RELATION TO ASPECTS OF BEHAVIOR. By Hudson Hoagland

neuroembryology. By Samuel R. DetWILER

THE EGGS OF MAMMALS. By GREgory Pincus

AUTONOMIC NEURO-EFFECTOR SYSTEMS. By Walter B. Cannon and Arturo RosenBLUETH

PHYTOHORMONES. By F. W. Went and Kenneth V. Thimann

DYNAMICS OF INFLAMMATION. BY VALX Menkin

VERTEBRATE PHOTORECEPTORS. By SAMUeL R. Detwiler 


\section{VERTEBRATE}

\section{PHOTORECEPTORS}

BY

SAMUEL R. DETWILER

Professor of Anatomy .

College of Physicians and Surgeons

Columbia University

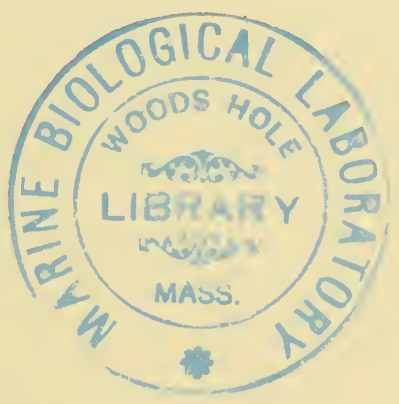

NEW YORK

THE MACMILLAN COMPANY 
Copyright, 1943,

BY THE MACMILLAN COMPANY.

All rights reserved - no part of this book may be reproduced in any form without permission in writing from the publisher, except by a reviewer who wishes to quote brief passages in connection with a review written for inclusion in magazine or newspaper.

Printed in the United States of America

Published_March, 1943 
To my wife

GLADYS 



\section{PREFACE}

The structure of the vertebrate retina in relation to its function is a subject about which much has been written. It might seem redundant, therefore, to add to the voluminous literature already extant. This is especially true in view of the recent appearance of two excellent books on the subject. The first of these, by S. L. Polyak, appeared in October 1941, while my writing was in progress; the second by G. L. Walls, which was published in August 1942, did not appear until after my manuscript had gone to the publishers. Despite the admirable and comprehensive accounts by these two authors, as well as previous shorter accounts by other writers (Cajal, 1894; Greeff, 1900; Garten, 1907; Franz, 1913; Arey, 1928; Kolmer, 1936 and others), I have been urged by my colleagues to finish my story, with the assurance that this monograph would adequately fulfill its originally intended purpose as a handbook for those interested in the visual apparatus of vertebrates.

It has been my plan to bring together in this volume certain essential structural and functional features of the retina, particularly of the photoreceptors. It has not been my purpose to enter into the minutiae of histological detail, for this would befog my original point of view which has been to present an account of the retinal photoreceptors in such a form as to be of value to those who have a general interest in the biology of the retina, rather than to the specialist. It is hoped, however, that it may be of definite service also to those more specifically concerned with problems of retinal physiology.

Since it has been my design to make this monograph relatively brief and readable, many omissions have been necessary, particularly with respect to the vast literature on the subject under discussion.

New York City

S. R. D. 


\section{ACKNOWLEDGMENTS}

The author desires to acknowledge his gratitude to his colleague, Dr. Leona Zacharias; to his secretary, Miss Dorothy Phillips; to his assistant, Mrs. William Seitz, for their aid in the preparation of the manuscript and in proofreading; and to Mr. Sidney Shapiro who prepared many of the illustrations. He gratefully acknowledges his thanks to Dr. Selig Hecht, Dr. W. J. Crozier, and Dr. Gregory Pincus of the editorial board of the Experimental Biology Monograph Series for reading the manuscript and for their helpful suggestions and constructive criticisms.

Acknowledgment is also given for the kind permission to reproduce other authors' figures from the following sources:-

Chicago University Press, Chicago, Illinois

J. \& A. Churchill, Ltd., London, England

Williams \& Wilkins Company, Baltimore, Maryland

Cambridge University Press, The Macmillan Company, New York

Rockefeller Institute for Medical Research, New York The C. V. Mosby Company, St. Louis, Missouri American Medical Association, Chicago, Illinois Wistar Institute of Anatomy \& Biology, Philadelphia, Pennsylvania

Paul B. Hoeber, Inc., New York 


\section{TABLE OF CONTENTS}

CHAPTER

I. THE VERTEBRATE EYE . . . . . . . 1

The Bulb

Sclera

Cornea

Uvea

Choroid and tapetum

Ciliary body

Iris

Lens

II. THE RETINA • . . . . . . . . 16

Pigment Epithelium

Pars Optica Retinae

III. THE STRUCTURE OF VISUAL CELLS . . . 37

The duplicity theory

Structure of rods

Structure of cones

The basis for rod and cone classification

IV. DIFFERENTIATION OF RETINAL ELEMENTS • 48

Development of human retina

Development of amphibian visual cells

Development of rat visual cells

V. RETINAL STRUCTURE AND ANIMAL HABITS . 60

The double retina

The retina of diurnal animals

The retina of nocturnal animals

VI. PHOTOMECHANICAL RESPONSES IN THE RETINA 74

Pigment migration in vertebrate eyes

Movements of rods and cones

Factors influencing photomechanical movements

Diurnal rhythms 
VII. ON THE SIGNIFICANCE OF PHOTOMECHANICAL MOVEMENTS . . . . . . . . . . 86

Theoretical considerations

VIII. THE RELATION OF RETINAL STRUCTURE TO

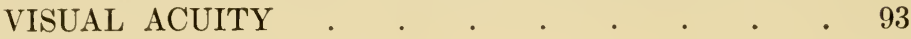

Rod and cone thresholds

Area centralis retinae

The fovea centralis

IX. ON THE SUPPOSED EVOLUTIONARY RELATIONSHIPS AND FUNCTIONAL SIGNIFICANCE OF THE FOVEA . . . . . . 108

X. RETINAL PHOTOPIGMENTS . . . . . 120

Photochemical theory of Hecht

The rhodopsin cycle

The porphyropsin cycle

Cone photopigment

Kolmer's "droplets" and the rod visual cycle

XI. VITAMIN A-DEFICIENCY AND THE RETINA . 143 Alteration of visual thresholds

Retinal damage and repair

XII. RECAPITULATION . . . . . . . . 160 BIBLIOGRAPHY . . . . . . . . 165

AUTHOR INDEX . . . . . . . . . 179

SUBJECT INDEX . . . . . . . . . 181 



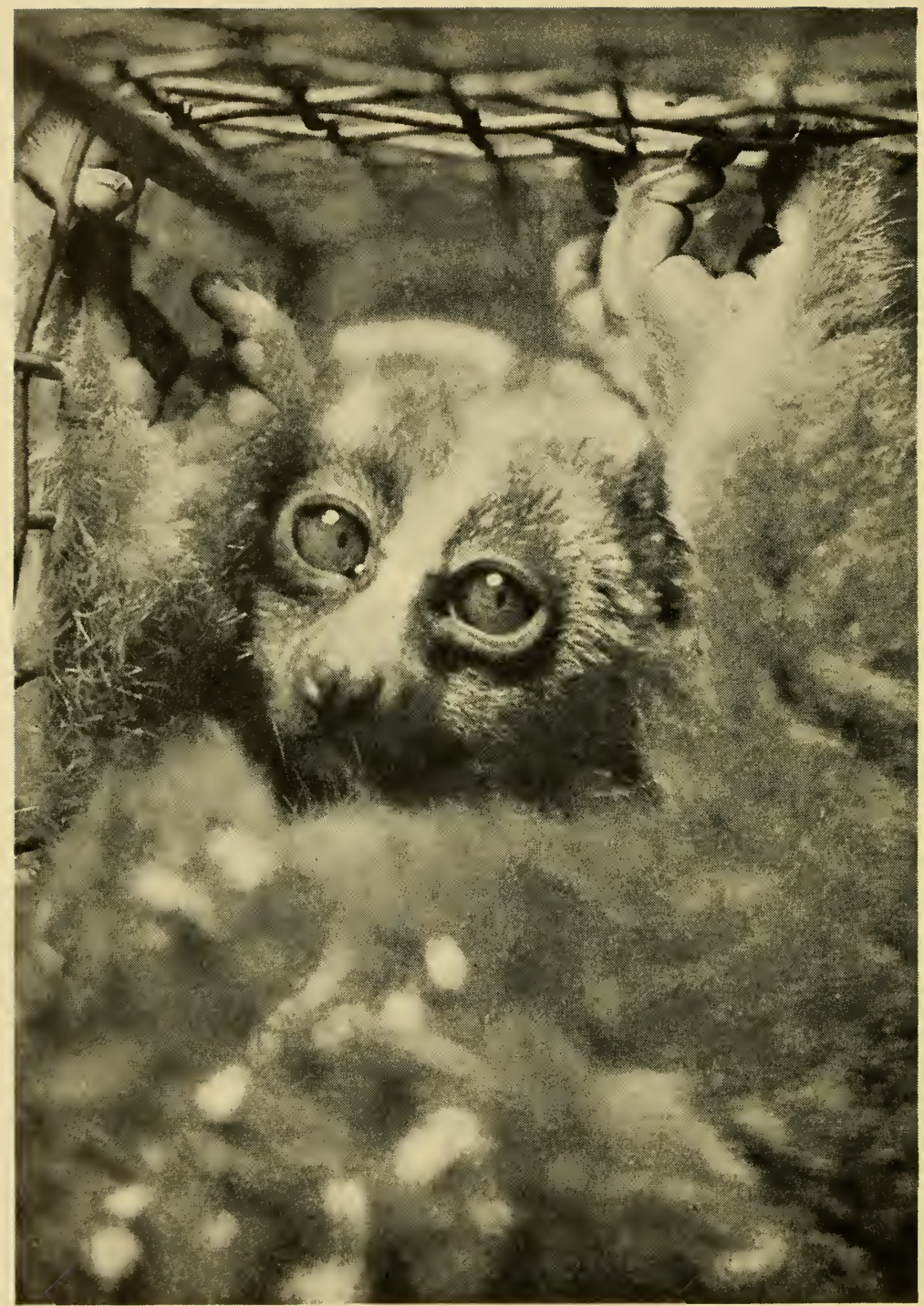

FIG. 1. Photograph of the slow moving Loris (Nycticebus tardigradus). 


\title{
VERTEBRATE PHOTORECEPTORS
}

\author{
CHAPTER I \\ THE VERTEBRATE EYE
}

It is well recognized among biologists that the eyes of vertebrates are constructed upon a common generalized plan. Despite the architectural similarity which one meets when studying the structure of eyes, one cannot help but be impressed with the fact that there are many deviations from the common scheme. This pertains not only to the sensory receptor and nervous transmitting mechanisms, but to the light-collecting and focusing devices as well.

Perhaps the most common characteristics of the vertebrate eye are the mode of origin of its essential parts, the presence of three concentric layers or tunics (tunica fibrosa, tunica vasculosa and tunica interna, and their respective component modifications), and the existence of an inverted retina, so that the photosensitive elements are turned away from rather than towards the source of illumination. This last condition has stimulated speculation among many students of biology, and various theories have been advanced which aim to account for the origin and inversion of the retina during the course of phylogeny. These theories have been reviewed recently in an article by Walls (1939b) who also presents his own theory.

Although our chief interest in the following pages will be concerned with the retina, and in particular with the visual cells themselves, it seems expedient by way of introduction to give a brief account of the bulb as a whole, which will be based primarily upon the human eye. No attempt will be 
made to describe the histological structure of the various parts of the bulb because of excellent descriptions found in various textbooks of histology and treatises on ophthalmology.

\section{The Bulb}

The human eyeball (bulbus oculi) is made up of the segments of two spheres, a scleral segment and a corneal segment - the former having a radius of $12 \mathrm{~mm}$. and the

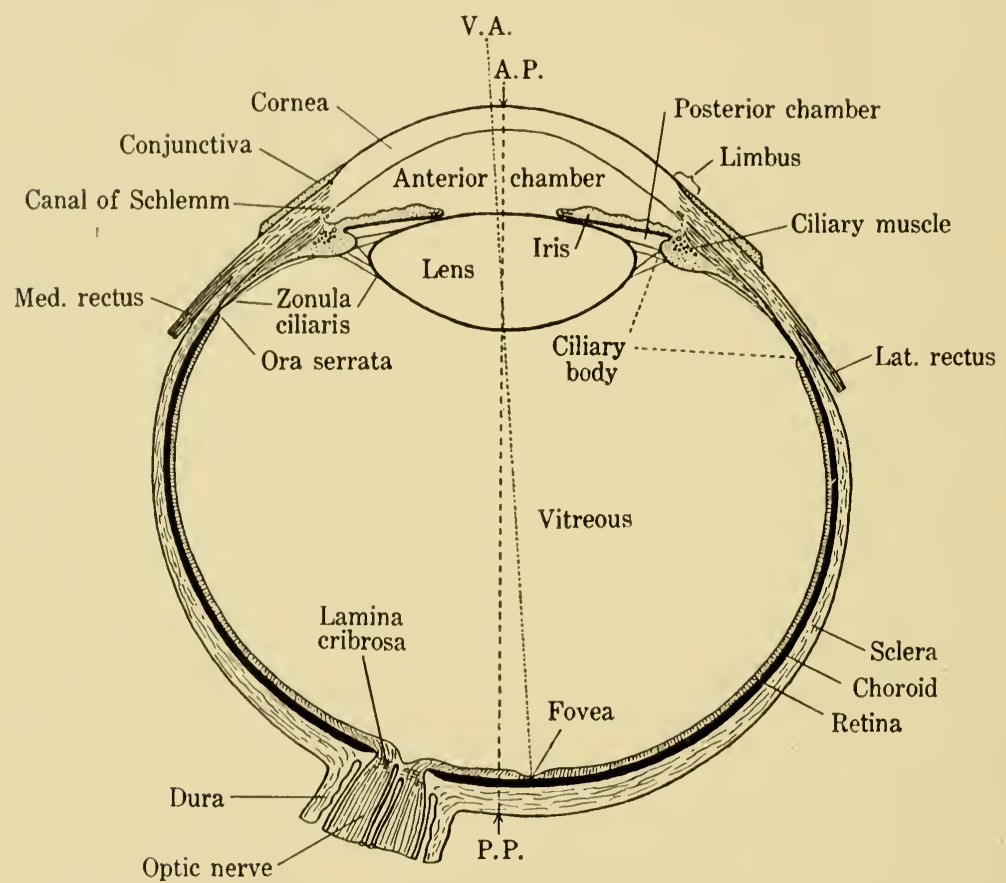

Fig. 2. Schematic horizontal meridional section of the human right eye. $\times 3$, A.P., anterior pole; P.P., posterior pole; V.A., visual axis. (From Bailey. Textbook of Histology, 10th Edition, The Williams \& Wilkins Company, Baltimore.)

latter a radius of $8 \mathrm{~mm}$. (Figure 2). At the junction of the two segments which are continuous structurally, there is a shallow circular furrow known as the external scleral sulcus. The anterior (corneal) segment occupies about onesixth of the surface of the bulb, which is relatively very 
much smaller than in many animals, particularly nocturnal forms (e.g. birds, rodents, lemurs) as will be pointed out later.

Whereas the dimensions of the bulb are relatively constant for individuals of the same species, the vertebrate eye varies enormously in its size. Fishes and birds have large eyes. In amphibians and reptiles they are small. Nocturnal lemurs have characteristically large eyes as have also deep sea fishes, and the eyes of the owl are said to occupy one-third the size of the head (Figure 3).

Whereas mammals vary greatly in size, there is no absolute correlation between body and eye size. DukeElder (1939) points out that size seems to be correlated to a greater degree with the nimbleness of the animal's movements and its necessity for acute vision rather than its bulk - giving as an ex-

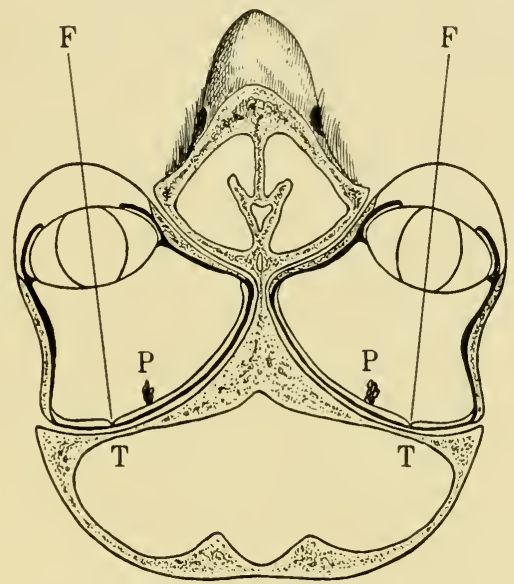

FIG. 3. Section through the head of the great horned owl (Bubo virginianus). FT, visual axis of the single, temporal fovea; $\mathrm{P}$, pecten. (Redrawn from figure 4, Casey Albert Wood, The Fundus Oculi of Birds, The Lakeside Press, Chicago.)

ample the antelope whose eye is larger than that of the elephant. It is also interesting to note that a meridional section of the hawk eye (unidentified species) in my own collection is almost as large as a corresponding section of an adult human eye - illustrating again the lack of correlation between the size of the eye and that of the body.

Vertebrate eyes vary but little in general shape, although there are some deviations. Deep sea fishes possess tubular eyes which are extremely long in the antero-posterior dimensions. The owl's eye is also of this general shape but not so extreme. Many nocturnal animals have somewhat elongated eyes by reason of their large and greatly curved corneas (e.g. nocturnal lemurs, Figures 48, 49). 


\section{Sclera}

The tunica fibrosa or outer layer of the eye comprises the cornea and sclera. The sclera forms the posterior five-sixths of the protective outer tunic. It is a tough membrane made up in man and in higher animals of dense fibrous connective tissue - mostly of collagenous fibers, but containing also some elastic fibers (Figure 4). Where the optic nerve fas-

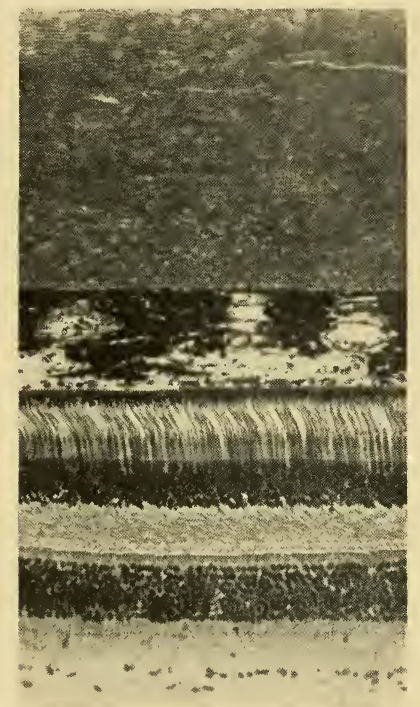

FIG. 4. Photomicrograph showing fibrous sclera of the monkey eye (Macaca mulatta). $\times 115$.

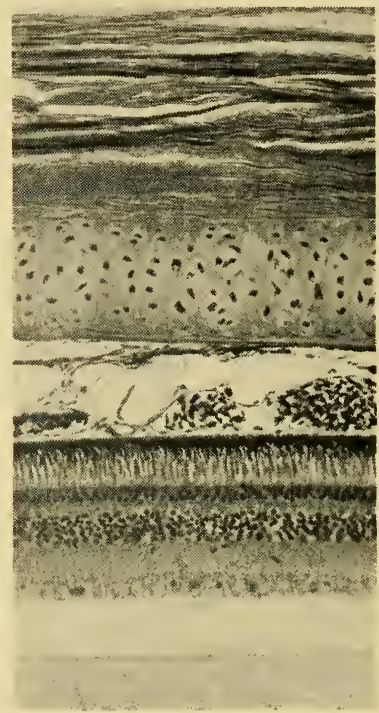

FIG. 5. Photomicrograph showing cartilaginous sclera of the hawk eye. $\times 115$.

ciculi leave the eye, this tunic is perforated and this region is known as the lamina cribrosa. The sclera is pierced also by nerves and blood vessels entering and leaving the bulb. In fishes, amphibians, reptiles, and birds the sclera is made up partly or entirely of hyaline cartilage. In fishes the cartilage is present usually in the form of laterally situated plaques. In amphibians, reptiles, and birds, the cartilage is continuous around the eye (Figure 5). In some forms the sclera is made up largely of bone (e.g. whale, and swordfish), and in other forms, varying degrees of calcification of 
the cartilage may occur (e.g. elasmobranchs, teleosts). In the anterior portion of the sclera of the hawk, bone is present. A similar condition exists in the owl's eye (Figure 6).

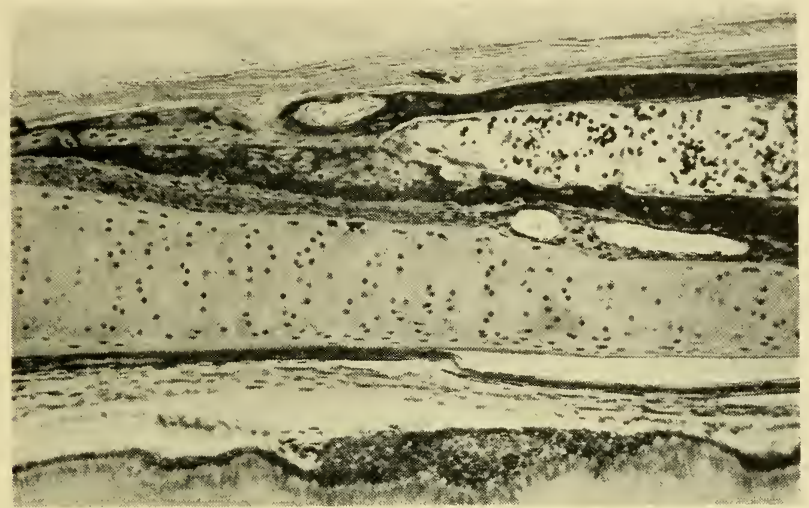

FIg. 6. Photomicrograph showing presence of bone in the anterior portion of the cartilaginous sclera of the owl eye. $\times 115$.

\section{Cornea}

The transparent cornea forms the anterior portion of the tunica fibrosa. In man it constitutes the anterior one-sixth of the eye and is more curved than the scleral portion. Viewed from in front it is elliptical - viewed from behind it is circular. The anterior surface is not uniformly curved; in the central portion it is spherical whereas towards the periphery it becomes flattened, especially towards the nasal side. Many measurements have been made of corneal curvatures in man (Duke-Elder, 1939). It is also a noteworthy fact that the refractive power of the human cornea is about two and one-half times that of the lens so that most of the refraction of light takes place at the corneal surface rather than in the lens itself.

The cornea varies in its relative size among vertebrates. In general it may be said that it is large in fishes and mammals, and small in reptiles and birds (excluding nocturnal forms). Apparently all nocturnal forms have large corneas which of course is an advantage for collecting the small 
amount of light. Perhaps the most striking cases of large corneas occur among the nocturnal lemurs where they occupy approximately one-third of the perimeter of their bulbs (Detwiler, 1940) (Figure 49).

\section{Uvea}

The tunica vasculosa (uvea) is made up of the choroid, ciliary body, and iris. It is characterized by the presence of abundant blood vessels and pigment cells. By reason of the heavy pigmentation of this layer, the bulb is light-tight except through the pupil. When the sclera is removed from the eye, this middle heavily pigmented tunic resembles the inside of a purplish grape skin, the pupil representing the point where the stem is pulled out - hence the name uvea or uveal tract.

Choroid. The choroid, which forms the posterior part of the uvea, is highly vascular, resembling somewhat in this respect the chorion of the foetus - hence the name choroid. The extreme vascularity of this region including the large spaces occupied by the veins makes this structure resemble erectile tissue. This layer is believed to share in the regulation of intraocular pressure. The choroid is intimately bound or attached to the epithelial pigment layer of the retina (tapetum nigra). In retinal detachment, the split occurs between the neural layers of the retina, and the epithelial pigment layer so that the latter remains adherent to the choroid.

The choroid varies considerably in thickness in various vertebrates. It shows also some noteworthy specializations. In some teleost fishes a silvery membrane is found between the suprachoroid and the vascular layer. This is formed of specialized cells containing crystals of guanine. The membrane extends into the iris and presents a characteristically silver luster. In some cartilaginous fishes and in some mammals there is present a reflecting layer known as the tapetum lucidum. It is situated between the choriocapillaris and the vessel layer in the posterior region of the eye, usually above 
and lateral to the optic disc. In this region of the mammalian eye, the pigment of the epithelial pigment layer is either sparse or lacking, hence incident light falls directly upon the tapetum and is reflected. Due to the presence of an overlying layer of doubly refracting crystals and to interference phenomena caused by the stratified formation and surface irregularities, an iridescent effect is produced. The eye of the animal thus shines with a peculiar luster in subdued light. The tapetum of carnivors is made up primarily of brick-like cells and is called the tapetum cellulosum. In herbivors it is made up of fibers - hence the name tapetum fibrosum. A very prominent fibrous

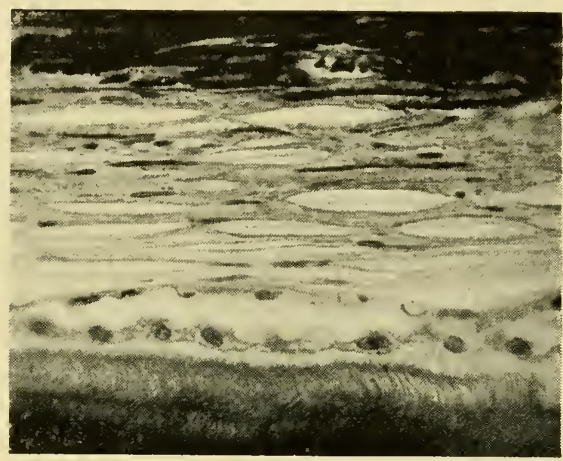

FIG. 7. Photomicrograph showing fibrous choroidal tapetum of the lemur, Nycticebus tardigradus. $\times 300$.

tapetum is present in the eyes of the nocturnal lemurs (e.g. Galago and Nycticebus, Figure 7), but in the nocturnal monkey (Nyctipithecus) it is absent. Nocturnality does not necessarily imply the presence of a tapetum, for in the gecko, which is nocturnal in its habits, it is absent. It is absent also in the eyes of apes and man.

Ciliary Body. In front of the ora serrata (Figure 2), the uveal tract becomes thickened and modified to form the ciliary body which runs around the eye as an asymmetrical girdle. In section it is essentially triangular in shape, the apex of which is continuous with the choroid. It forms the attachment for the suspensory ligament of the lens and gives origin, also, to the iris. The inner (vitreal) surface of the ciliary body presents two zones. The posterior two-thirds is deeply pigmented and relatively smooth. This is the orbiculus ciliaris or pars plana. The anterior third bears, in man, from 70 to 80 radially arranged ridges - the ciliary processes. 
This region is called the corona ciliaris (pars plicata). The vascular ciliary processes are covered over by two layers of epithelium. The inner heavily pigmented layer is a continuation of the pigment epithelium of the retina. The outer epithelial layer is a continuation of the sensory portion of the retina. These two layers constitute the so-called pars ciliaris retinae. They continue over the posterior surface of the iris as the pars iridica retinae.

Within the ciliary body is the ciliary muscle which, in man, is divisible into three regions: a) meridional fibers (Brücke's muscle), b) radial fibers, and $c$ ) circular fibers (Müller's muscle).

This muscle, which is concerned with accommodation, varies considerably in shape and size. When the circular fibers are strongly developed or in the contracted state, the muscle appears shorter (hypermetropic type). When weakly developed or relaxed, the muscle appears larger and flatter (myopic type).

The ciliary body varies greatly among vertebrates. In fishes and amphibians the ciliary processes are rudimentary. They are well developed in reptiles and in birds. In the latter the processes are large and numerous (pigeon, 104-108; horned owl, 130; kestrel, 158, v. Hess, 1909). The fine accommodating power of birds is well known. In mammals apparently the processes vary considerably in size. For example, they are said to be small in the rabbit; large in the ox.

The ciliary muscle, also, shows marked variations in size. It is extremely small in fishes, and contains only a few muscular fibers. Amphibians possess only a rudimentary bundle of meridional fibers. Birds have a very large muscle which is striated. In some instances the muscle inserts into a bony ring around the sclero-corneal margin. According to DukeElder, this muscle in mammals exhibits an ascending scale of development. It is least developed in rodents, larger in herbivors, still more developed in carnivors and reaches its highest development in primates. Although striated in reptiles and in birds, this muscle in mammals (as in fishes) is of 
the smooth type. It is interesting in this connection to point out that the ciliary muscles of nocturnal lemurs (Galago and Nycticebus) are strikingly large. Both these forms, however, have large lenses. When one compares the relative size of the ciliary muscle with the size of the whole eye, one finds that in Galago it is relatively four times as large as in the rhesus monkey (Macaca mulatta) and in man, and nearly twice as large as in the marmoset (Detwiler, 1939). In Nycticebus this muscle is larger than in man, yet the eye as a whole is only slightly more than one-third as large (Figures $8,9,10,11)$. If the size of the muscle can be taken as significant, then it would seem that the accommodation mechanism of the lemur is superior to that of man.

Although nocturnal animals in general possess large lenses and correspondingly large ciliary muscles, a noted exception is seen in the gecko where the lens is enormous, but where the ciliary muscle is extremely small. It is apparent then that a large lens does not necessarily connote the presence of a correspondingly large ciliary muscle.

Iris. The iris, which is attached to the anterior surface of the ciliary body, forms a delicate diaphragm across the anterior part of the eye. It possesses an opening, the pupil, which is usually somewhat eccentrically placed towards the nasal side. The pupillary border rests upon the lens. The iris does not have the shape of a flat diaphragm but inclines forward from the ciliary body in the form of a low truncated cone.

The iris houses two muscles: the m. sphincter pupillae and the $\mathrm{m}$. dilatator pupillae. Both have the same embryonic origin, being derived from the pigment epithelial layer of the retina. In the former, the epithelial cells are completely converted into smooth muscle fibers; in the latter, the transformation is incomplete so that these cells can justly be called "myoepithelial cells."

Since the size of the pupil is constantly varying with respect to the amount of light and changing accommodation, the width of the iris is likewise very inconstant. 

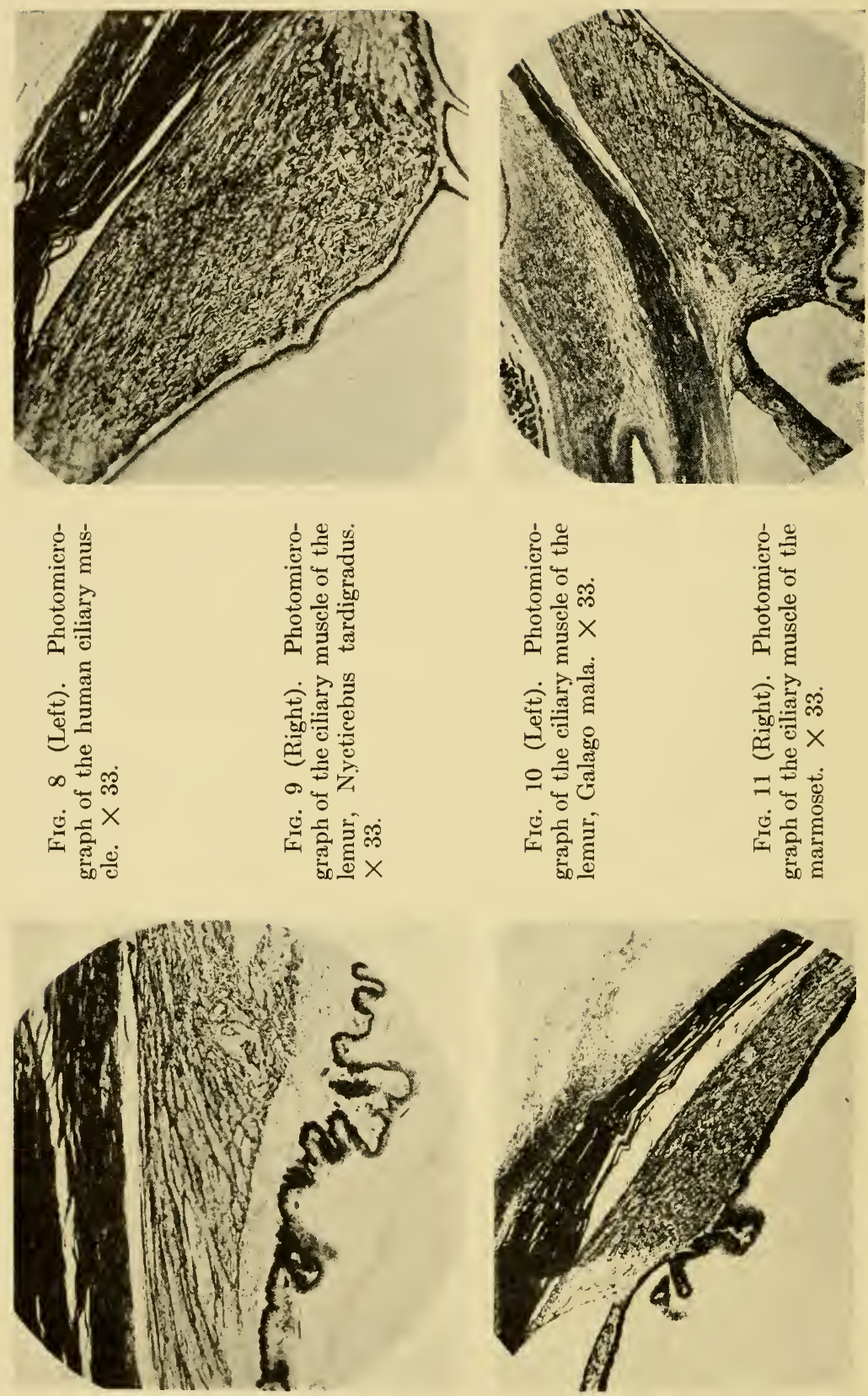
The iris varies with respect to its color in different individuals. The posterior border (retinal portion) is heavily pigmented. In white races, the stroma is relatively free from pigment at birth. Light piercing the transparent anterior layers is reflected back and owing to the phenomenon of interference, the color appears blue. If no pigment develops in the anterior border layers, the iris remains blue except in the regions occupied by blood vessels which are not transparent. With moderate amount of pigment developing in the anterior border layer, the color becomes gray; with the development of much pigment in this layer the color becomes brown.

In connection with variations in the iris it is of interest to quote from Duke-Elder (1939). "So great are the individual variations of the architecture of the iris that these were long ago suggested as a basis for the identification of criminals (Bertillon, Rev. Sc., XXXVI, 65, 1885), a practice which has lately been revived. Nor has imagination and superstition failed to clothe it with significance; Tenon saw the letter $\mathrm{T}$ in many, while the name of the Emperor Napoleon or other characters more mystical used to be deciphered. Even today an extraordinary cult with a considerable following and a voluminous literature flourishes, particularly in Central Europe, the devotees of which attribute to each minute area of this tissue an affinity for a different organ in the body, in the list of which the eye itself is included, and from an examination of the appropriate area the initiated can diagnose any disease to which flesh may fall heir. They call themselves oculodiagnosticians ('irido-diagnosis,' 'Augendiagnose,' etc.). (See Gilbert, 1924; Mondain, Tilmont and Benoit, 1924)."

In this connection it might be of interest to cite the extraordinary case of John Woods which is reproduced on the following page from Ballantyne's (1904) Antenatal Pathology and Hygiene (Figure 22). 


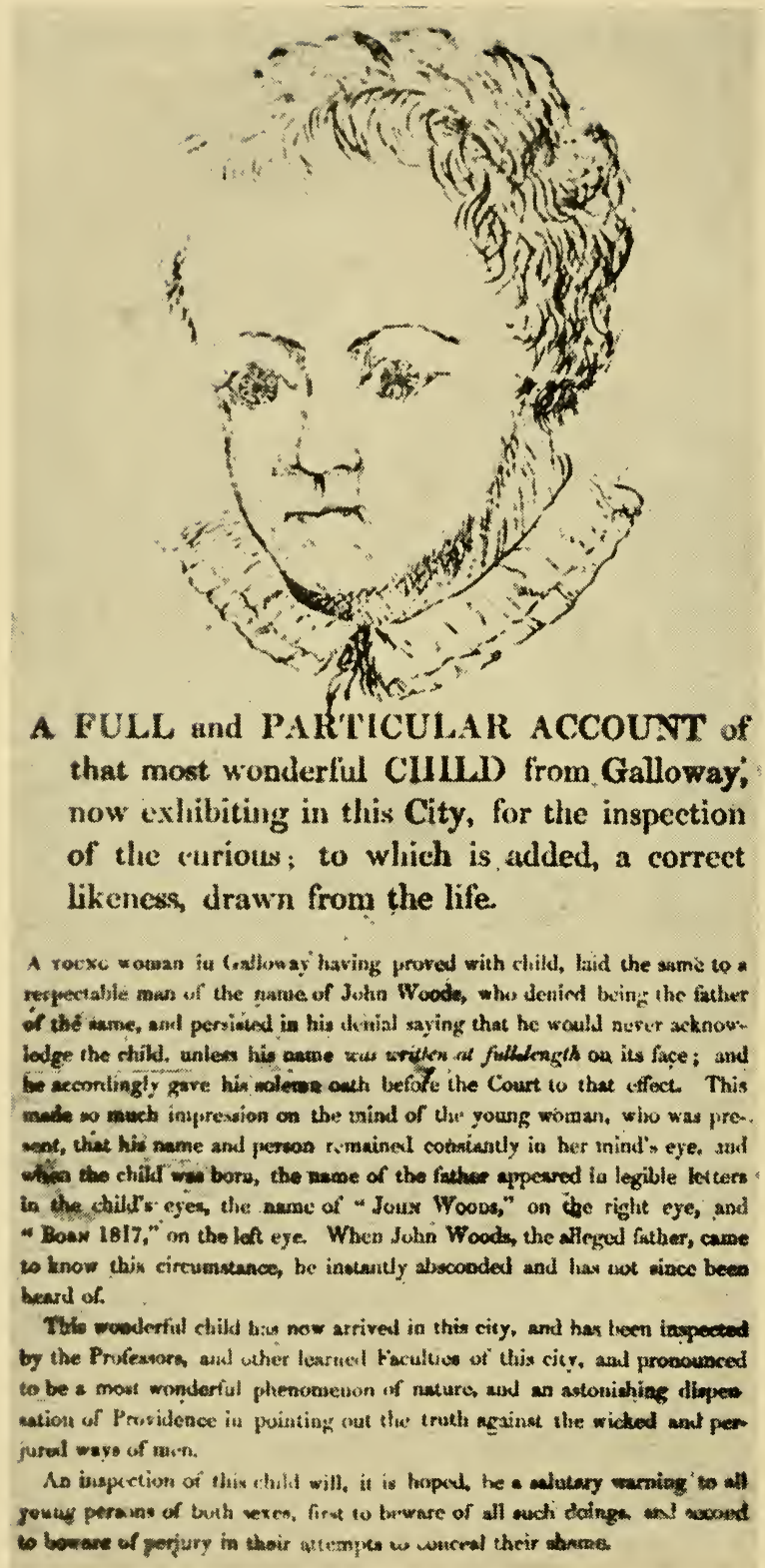




\section{LENS}

The human lens is a biconvex structure with a rounded border (equator). The anterior surface is curved as a flattened ellipsoid; the posterior surface is more highly curved as a paraboloid. It is placed behind the pupil and its anterior surface is adjacent to the posterior surface of the iris. It is supported by the zonular fibers and its equator is separated from the ciliary body by a free interval $0.5 \mathrm{~mm}$. wide. Numerous measurements of the equatorial and the sagittal diameters of the lens have been made in individuals of all ages. These are listed in tabular form by Duke-Elder (1939) to whom the reader is referred.

The lens in all classes of vertebrates has a common origin, viz., from an invaginated lens placode in the surface epithelium. In this sense then the lens in all vertebrates conforms to the same general plan. As one ascends the evolutionary scale from fishes to man, one can say in general that the lens becomes progressively less spherical and more biconvex. This does not hold for aquatic forms in which the spherical shape is maintained. Such forms require a greater refracting power than terrestrial and avian forms. This is due to the fact that the refractive index of the cornea and that of water are about equal, a condition which practically eliminates the converging power of the cornea.

FIG. 12. Diagrammatic section through the eye of the marine fish, Serranus scriba. Fo, fovea. (Photographed from Kahmann, 1936. v. Graefe's Arch. f. Ophth., Bd. 135.)

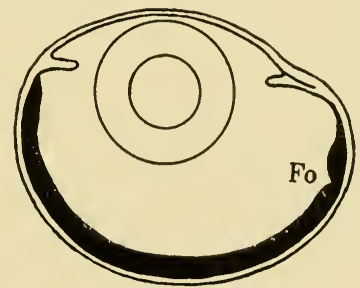

In fishes the lens tends to be almost spherical and may protrude into the anterior chamber so as almost to touch the cornea (Figure 12). In amphibians the anterior and posterior surfaces become somewhat flattened so that an ovoid shape results. In reptiles and birds, the convexity becomes 
less and less. In mammals and primates, with some exceptions, the anterior and posterior surfaces are even flatter.

Despite the general tendency for the lens to become less spherical as one ascends the vertebrate scale, there are many exceptions. The retention of a spherical lens by aquatic forms has already been pointed out. The lenses of nocturnal

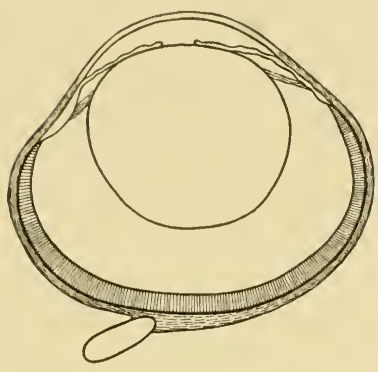

Fig. 13. Diagrammatic section of the eye of the gecko (Gecko swinhonis Güenther) showing large, almost spherical lens. $\times 11$. forms, regardless of class, also tend towards spheroidal forms. One of the classic examples is the gecko (Figure 13) in which the lens is seen to be almost a perfect sphere. It is to be noted from the figure that the lens is enormous in proportion to other eye structures. Its volume is equal to that of the vitreous, whereas in the human eye, the lens is about one-ninth the size of the vitreous. It is noteworthy in this connection that in the nocturnal lemurs the lens is not only much more biconvex than in primates, but it is relatively larger (Figures 49, 52 and Table 2). One exception to this is Nyctipithecus (owl monkey) which is nocturnal in its habits. Here too the lens is much more biconvex than in the diurnal monkeys, and resembles in shape and relative size that of the nocturnal lemuroids. We see, therefore, that nocturnal forms also possess greater refracting power than do diurnal forms. This of course is an advantage in helping to concentrate the small amount of light available.

Another interesting feature of the nocturnal eye, in addition to the large highly curved cornea and large spheroid lens, is the shape of the pupil. Whereas most animals have circular pupils, many nocturnal forms possess oval-shaped, frequently vertical (e.g. geckos, alligators, cats); sometimes horizontal (e.g. Tarsius) pupils. Hartridge (1919) in discussing the function of a vertical slit pupil such as in the cat, points out that an oval pupil in which the long axis is vertical will cause the lens system to form images in which 
the aberrations of horizontal contours are greater than those belonging to vertical contours. The contours of trees and their branches are principally vertical; therefore, if the image formed on the retina could be increased by sacrificing the definition of horizontal contours it would be an advantage. Interesting as this is, it seems doubtful whether the theory can be successfully applied. Certainly at night or in dim light the pupil of the cat is wide open and round. Furthermore, in daytime when the pupil is a vertical slit or oval, cats hunt and catch on the ground - in other words along horizontal contours. The alligator also hunts along horizontal contours, yet it has a vertical slit pupil. Whereas the nocturnal tree-dwelling lemurs have a vertical oval pupil, the nocturnal Tarsius which is also a tree dweller possesses a horizontal oval pupil.

Rochon-Duvigneaud (1917) in listing the characteristics of the eye of the geckos which adapt them to nocturnal vision, includes the form of the pupil - a vertical slit which shows a rapidity of movement surpassing that of the human eye and approaching that of birds. In dim light the pupil is a large oval, or even round, as in the cat. In bright light it is closed completely. According to him a round pupil can dilate as well as an oval, but it cannot be entirely closed. He believes that the chief function of an oval pupil, in an animal adapted to twilight vision, is to protect the eye against an excess of light. Presumably this is a perfectly adequate explanation for the presence of an oval pupil be it vertical or horizontal. My own experience with the gecko pupil, however, was that it never completely closed in bright illumination, but became reduced to several very narrow slit-like apertures. 


\section{CHAPTER II}

\section{THE RETINA}

The retina arises as an evagination of the forebrain. It subsequently becomes invaginated to form a doublelayered cup (optic cup). The outer layer forms the pigment epithelium (epithelial pigment layer) and is non-nervous. The inner layer forms the nervous portion of the retina (pars optica retinae).

Pigment Epithelium. The pigment epithelium comprises a single layer of regularly prismatic (usually hexagonal) cells. The outer portion of each cell (choroidal) rests upon the innermost layer of the choroid (lamina vitrea) and contains a spherical nucleus and a moderate quantity of pigment granules. The inner portion is filled with dark needleshaped pigment granules which extend into processes reaching down between the outer segments of the visual cells. The pigment epithelial cell in humans varies in diameter from 12 to $18 \mu$. In the region of the macula the cells become tall, more slender and very deeply pigmented. Towards the ora they are broader and lower.

The pigment is a melanin termed fuscin and varies greatly in amount in different animals. It is absent in albinos, in the eyes of elasmobranchs, in the fundus of nocturnal lemurs, and in the fundus of those mammals which possess a choroidal tapetum (Figure 7 ). It can easily be seen that the presence of epithelial pigment would be of great disadvantage to those animals which possess a choroidal tapetum for the reflection of light. Whereas many nocturnal animals are devoid of this pigment in the fundus of the eye, other nocturnal forms are heavily pigmented in this region (e.g. geckos). The epithelial pigment is abundant in bony fishes, anuran amphibians, and birds. It is especially heavy in birds, in fact to such an extent that visual cells cannot be seen in fixed preparations of light-adapted eyes without 
bleaching out the pigment. The same may be said of the teleosts. In reptiles, the amount of pigment varies considerably. Turtles possess a moderate amount, diurnal and nocturnal lizards are more heavily pigmented, and especially is this true of the fovea of diurnal forms. In the alligators and crocodiles, the melanin pigment varies in amount and distribution. Below and immediately above the optic nerve disc it is heavy. In the dorsal and posterior part of the eye, melanin pigment is almost absent, but instead, the cells here are filled with fine guanine granules which give this portion of the retina a glistening silvery appearance in contrast with the dark brown of the lower portion of the eye. This guaninecontaining portion constitutes a retinal tapetum in contrast with the pigment-free choroidal tapetum of mammals (Figure 14). A retinal guanine tapetum occurs also in numerous fishes (Wunder, 1926).

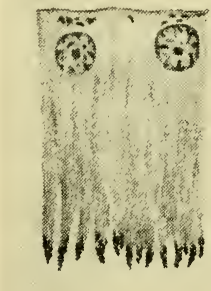

A

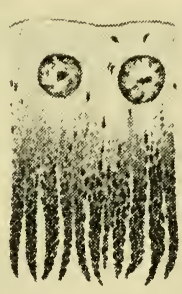

$\mathrm{B}$

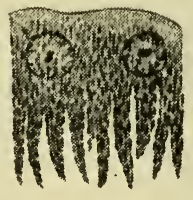

C

FIG. 14. Drawings of various regions of the epithelial pigment layer of Alligator mississippiensis. A, a portion of the tapetum showing broad zone of guanine, and a narrow vitreal border of melanin; $\mathrm{B}$, a portion of the pigment layer showing choroidal guanine-containing portion and vitreal melanin-containing portion; C, epithelial pigment layer beyond the region of the tapetum, showing dense melanin pigment and absence of guanine pigment. $\times 665$. (Redrawn from Laurens and Detwiler, 1921, J. Exp. Zool., v. 32.)

The significance of the epithelial pigment, its amount and migratory capacity in relation to vision or to adaptation of the eye, is a subject about which much has been written. This subject will be considered in detail in a later chapter.

Pars Optica Retinae. The retina in the more limited sense consists of a very delicate membrane covering the inner 
surface of the posterior part of the eye. It contains the photoreceptor elements, the nervous transmitting apparatus for vision, and a supporting framework. Excluding the pigment epithelium which is non-nervous, the retina is made up of nine layers arranged from without inward as follows (Figure 15):

1. Layer of visual cells (rods and cones)

2. External limiting membrane

3. External nuclear (granular) layer

4. External molecular (plexiform, reticular) layer

5. Internal nuclear (granular) layer

6. Internal molecular (plexiform, reticular) layer

7. Ganglion cell layer

8. Nerve fiber layer

9. Internal limiting membrane
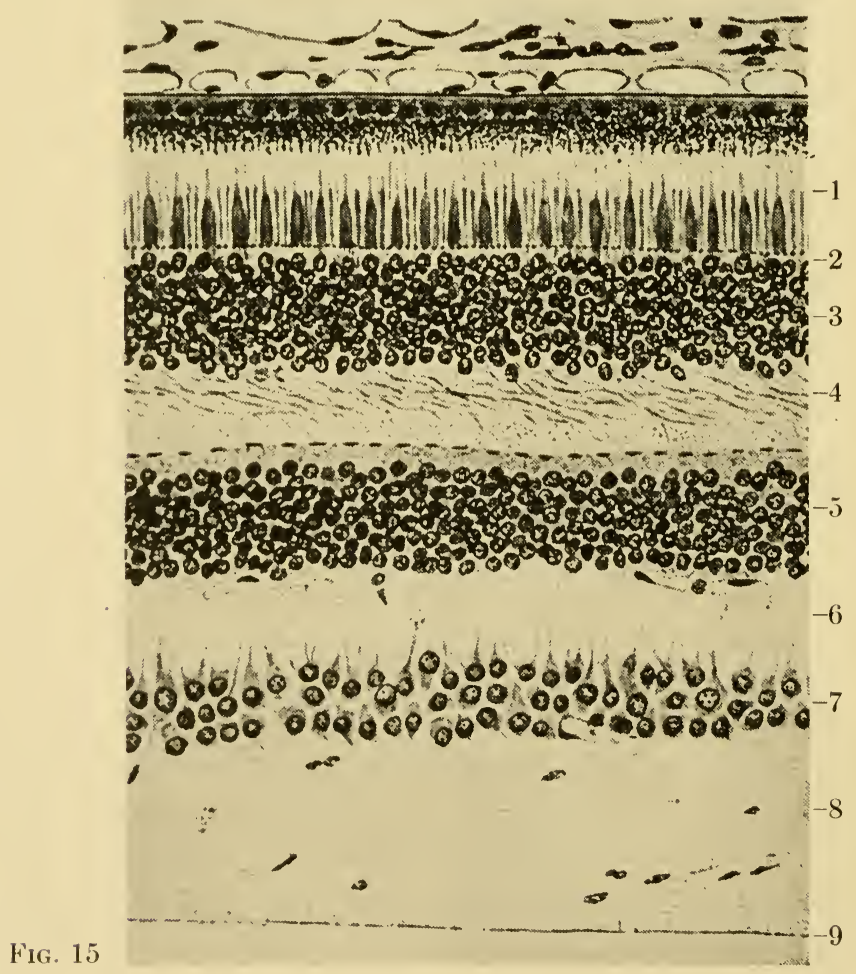
The first four layers listed above constitute the neuroepithelial layer and are the neurones of the first order. The remaining layers are considered the cerebral portion wherein there exists a complex arrangement of nervous and sustentacular elements resembling, in structure and function, those of the central nervous system of which the retina represents an outlying portion. The fifth layer (internal nuclear layer) which contains the bipolar, horizontal, and amacrine cells, and the sixth layer, comprise the neurones of the second order. The seventh and eighth layers make up the neurones of the third order, which pass centripetally to the primary optic center (lateral geniculate body) of the metathalamus.

The thickness of the retina varies considerably in different regions of the same eye, being typically thickest in the fundus (except at the region of the fovea), and thinnest at the ora serrata where the nervous portion of the retina terminates (Figure 2). Many measurements have been made of the thickness of the various retinal layers in man and in animals, with not too successful agreement among investigators. This is undoubtedly due to the fact that the retina, which is a most delicate membrane, is easily distorted by various histological techniques, so that more or less shrinkage invariably follows fixation. Hence the results may differ considerably. Menner (1929) made measurements of the retina in a number of representative vertebrates, the results of which differ widely from those of my own upon corresponding animals of the same classes of vertebrates. My measurements were made of the retinas of twentyseven animals from all classes of vertebrates. The eyes were preserved with the same fixative (modified Held's) so that the matter of differential shrinkage due to the use of different fixatives can at least be discounted. The measurements were made in the region of the fundus, approximately midway between optic nerve and ora serrata, and near (.5 to $1 \mathrm{~mm}$.) the ora serrata. These measurements include the following: 
Thickness of entire retina in $\mu$

Distance from lamina vitrea to external limiting membrane in $\mu$

Distance from external limiting membrane to distal tips of visual cells (rods and cones) in $\mu$

Thickness of the external nuclear layer in $\mu$

Thickness of the internal nuclear layer in $\mu$

It is only by making such measurements that one can get a true picture of the great variation in the thickness of the various retinal layers in different forms. The thinnest retinas in the region of the fundus of the forms studied were found to exist in the guinea pig $(130 \mu)$, the bat $(133 \mu)$, the hamster $(182 \mu)$, and the catfish $(182 \mu)$. The thickest were in the macular region of the chameleon $(442 \mu)$, the hawk $(400 \mu)$, the owl $(389 \mu)$, and the human $(390 \mu)$. The diurnal lizards and birds in general were found to have thicker retinas than mammals and the anthropoids.

The longest cones were found in the chameleon where the foveal cones measured $100 \mu$ (from distal tip to external limiting membrane), and in the marmoset $(91 \mu)$. The shortest cones were measured in the catfish $(31 \mu$, lightadapted). The longest rods were encountered in the teleost (Fundulus) where the light-adapted rods measured $99 \mu$ and in the owl $(83 \mu)$. The shortest rods were found in the bat $(76 \mu)$ and the alligator $(23 \mu)$.

Perhaps the most extreme differences were found to exist in the thickness of the external and internal nuclear layers. The external nuclear layer ranged from $10 \mu$ in Ameiurus (catfish) to $104 \mu$ in the nocturnal monkey (Nyctipithecus), although it was found to be almost as thick in the nocturnal lemurs (Galago $78 \mu$; Nycticebus $86 \mu$ ). The internal nuclear layer was found to range from $21 \mu$ in the dogfish to $122 \mu$ in the sparrow, and $166 \mu$ in the macular region of the hawk. These extreme differences (and others) are seen in Figure 16 and in Table 1.

The external nuclear layer of all nocturnal animals (except geckos) is relatively much thicker than the internal, 

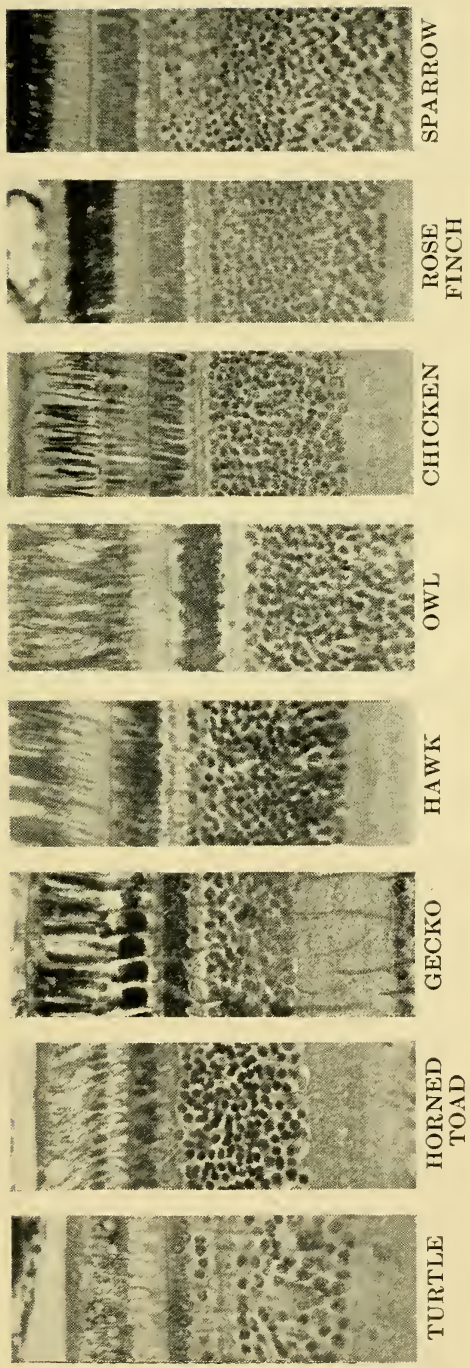
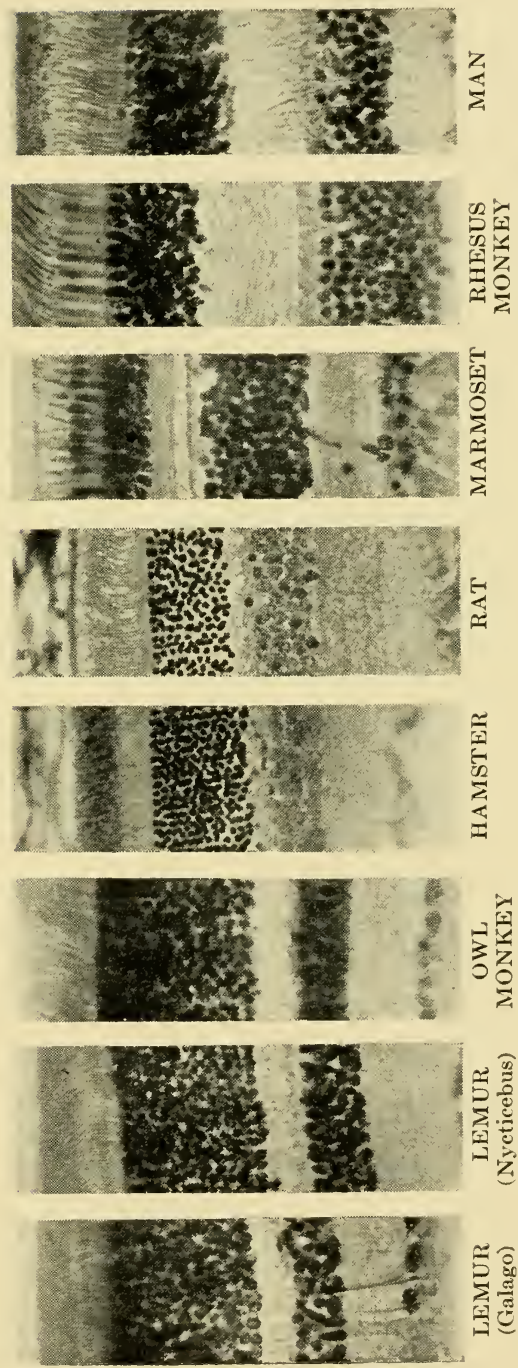


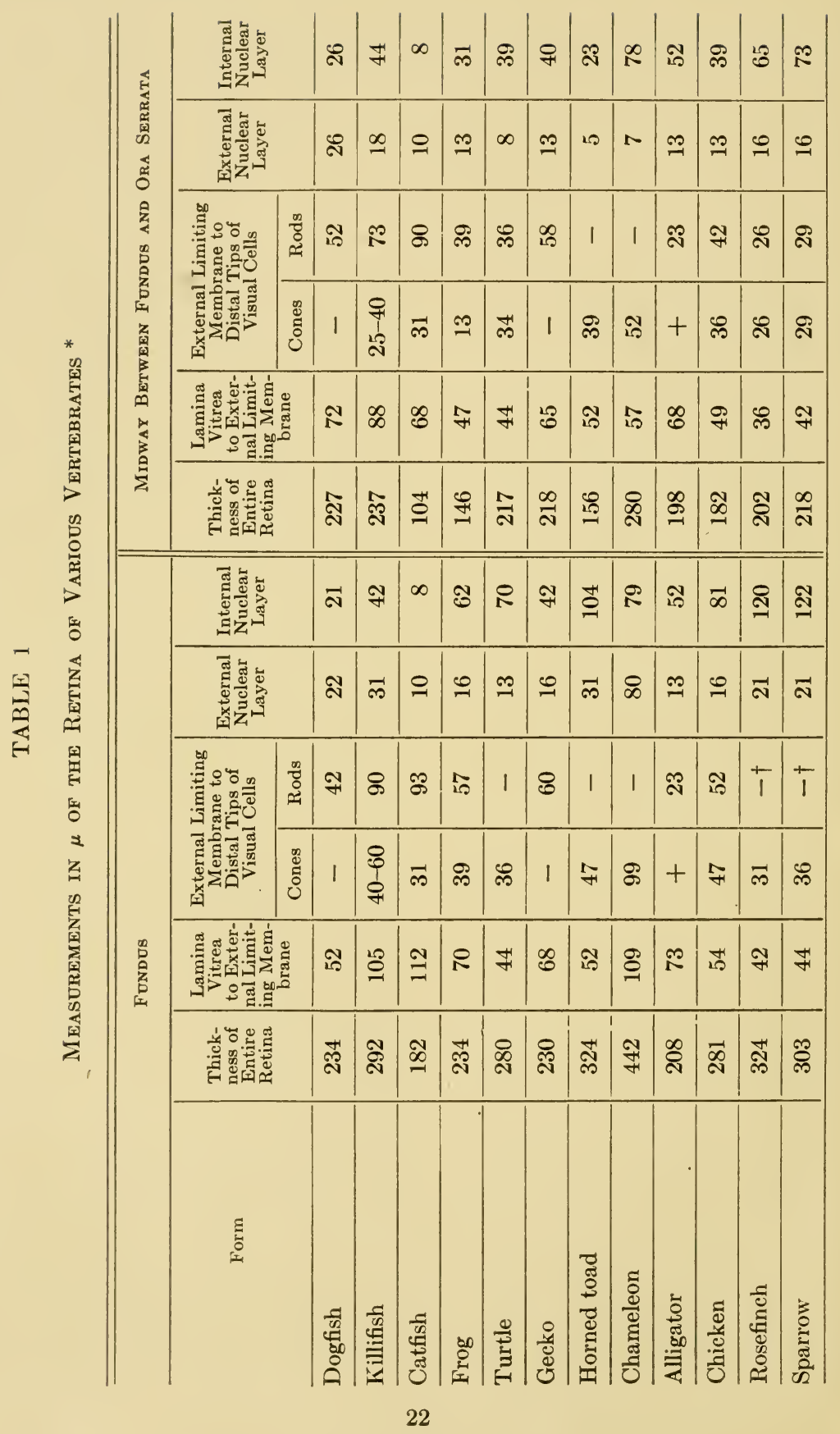




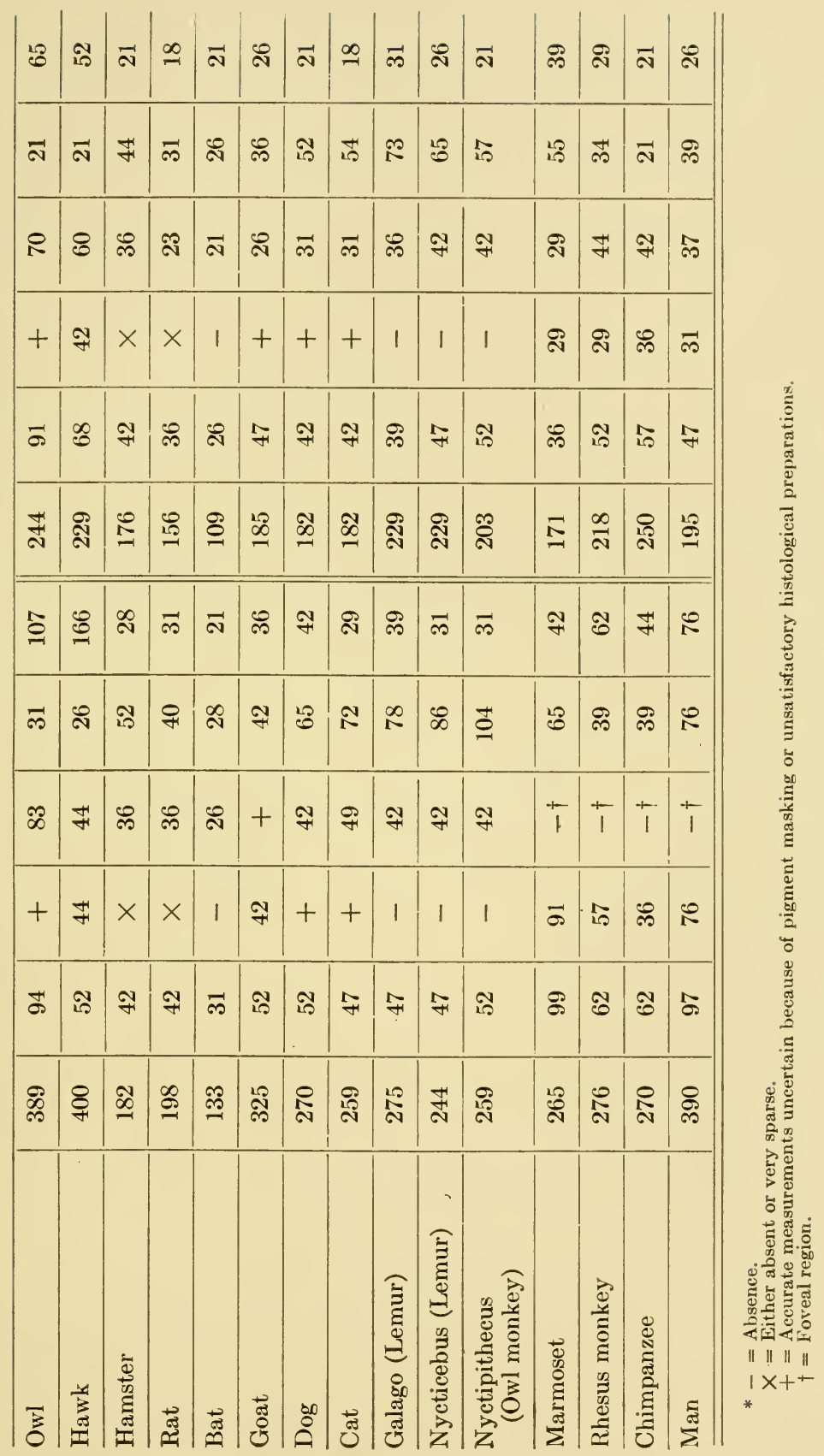


especially in the lemurs; whereas in the diurnal lizards, and particularly in birds, the internal nuclear layer is much thicker. This is striking in the sparrow and goldfinch (Figure 16). The great thickness of this layer in birds is in all probability an anatomical expression of their great visual acuity. In the nocturnal forms, the reverse is true, and many rods are collected into fewer bipolar and association cells, so that the external nuclear layer is much broader (thicker) than the inner nuclear layer (Figure 16).

It is apparent that the very thick internal nuclear layer of birds must contain a great many association cells. In the sparrow and rosefinch it is between five and six times as thick as the external nuclear layer. There are probably less bipolar cells than visual cells (since these forms have rods in addition to cones). Hence the greater part of this layer must be made up of other neurones (amacrine cells and horizontal association cells). In this connection it is of interest to quote from Casey Wood (1917) who says, "According to Franz (Das Vogelauge, 1911) the layers of the avian retina are more sharply defined than in any other vertebrate; even more so than in Mammals. There are no separate bipolar or optical ganglion cells in Birds, while we find in Mammals isolated amacrine cells, and, in reptiles, bipolar cells." This statement is difficult to understand for Franz (1913) certainly shows bipolar cells in the avian retina, and Wood himself (page 17) in speaking of the internal nuclear layer says, "Between, and further in than the cells, which Schiefferdecker calls nucleated cells, are found two kinds of bipolars; first, outer bipolars with highly developed dendrites, and internal, small or thin bipolars with weaker dendrites." The presence of bipolar cells in all classes of vertebrates is an established fact.

Although the earlier anatomists did much to unravel the detailed cellular architecture of the retina, it was not until the introduction of the chrome-silver method of Golgi and the methylene blue technique of Ehrlich that the finer structures and their connections were adequately worked 
out. Whereas the Golgi method was employed by Tartuferi (1887), and Dogiel (1888), we are indebted largely to the famous Spanish neurologist, Ramón y Cajal $(1893,1894)$ for unraveling the complexities of the finer nervous connections of the retina.

Franz (1913) published composite figures of the retinal neurones in the different classes of vertebrates. These were based upon the findings of earlier investigators (Retzius, Neumayer, Schiefferdecker, Schafer, H. Müller, M. Schutze, Aichel, Greeff, Krause, and especially Cajal). His plates have been redrawn with some modifications and are réproduced in Figures 17-22. These show the various types of bipolar, amacrine, horizontal, ganglion, and supporting cells. One important fact among others will be noted, viz., that in selachians, amphibians, reptiles, and diurnal birds, the rods, like the cones, make dendritic connections with the bipolar cells, whereas in teleosts, nocturnal birds, and mammals they end in knobs. This does not agree with Pütter's (1912) contention that rods and cones can be differentiated by their mode of connections with the bipolar cells. He claims that rods have centripetal molecular endings and the cones dendritic endings. If this were true, then what Franz has labeled "rods" in selachians, amphibians, and birds would have to be considered as cones - a fact which is not supported from other anatomical and physiological evidence.

Just lateral to the posterior pole and in the line of the visual axis, the retina in man, anthropoids, and some other forms undergoes a localized modification of its layers to form a funnel-shaped depression. In this area, which in man is termed the macula lutea because of its yellow pigment, the inner layers of the retina are spread apart, leaving in the center a small pit, called the fovea centralis (Figure 23). In this region there are no rods and the retina is characterized by the presence of long slender closely packed cones. It is the region of highest visual acuity and serves the function of central vision. For the human eye, Duke-Elder describes three regions as follows: 


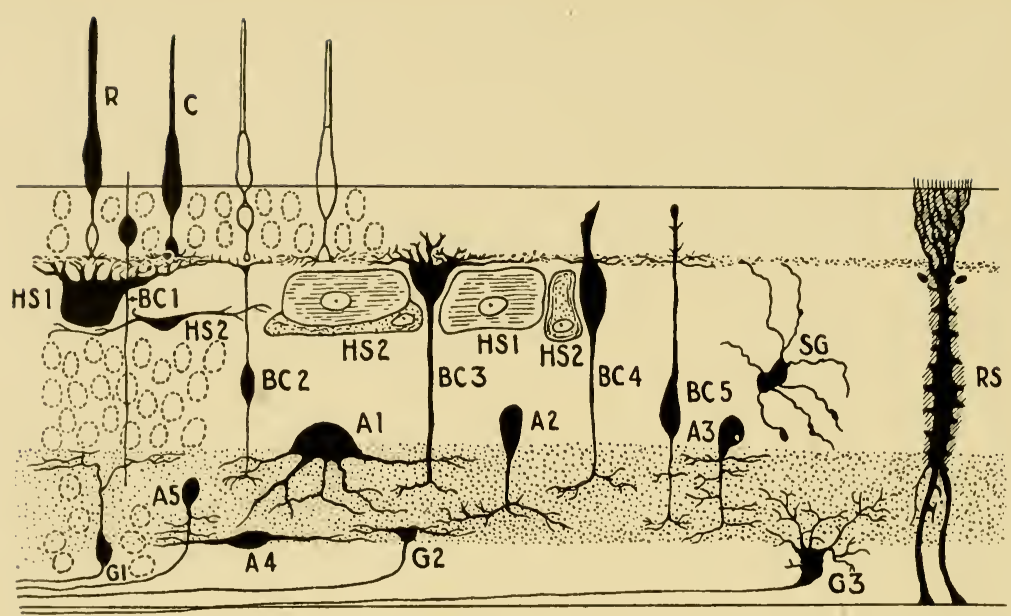

FIG. 17. Composite Golgi preparation showing the different elements of the selachian retina. Redrawn and relabeled from Franz, 1913 (Oppel's Lehrbuch). R, rod; C, cone; BC1, displaced bipolar cell, BC2-BC5, bipolar cells; HS1, HS2, horizontal supporting cells; SG, stellar ganglion cells; A1-A4, amacrine cells; A5, "nervous" amacrine cell; G1-G3, ganglion cells; RS, radial supporting cell (Müller fiber).

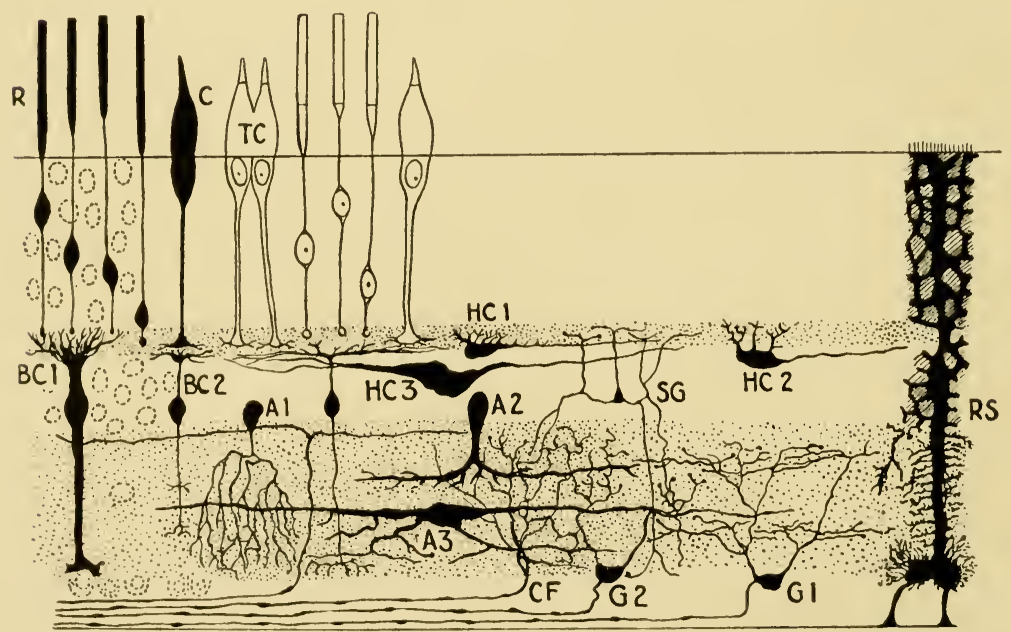

FIG. 18. Composite Golgi preparation of the teleost retina. Redrawn and relabeled from Franz, 1913. R, rod; C, cone; TC, twin cone; HC1-HC3, horizontal cells; BC1, rod bipolar cell; BC2, cone bipolar cell; SG, stellar ganglion cell; A1, diffuse amacrine cell; A2, "layered" amacrine cell; A3, dispersed amacrine cell; G1, G2, ganglion cells; RS, radial supporting cell (Müller fiber); CF, centrifugal nerve fiber. 


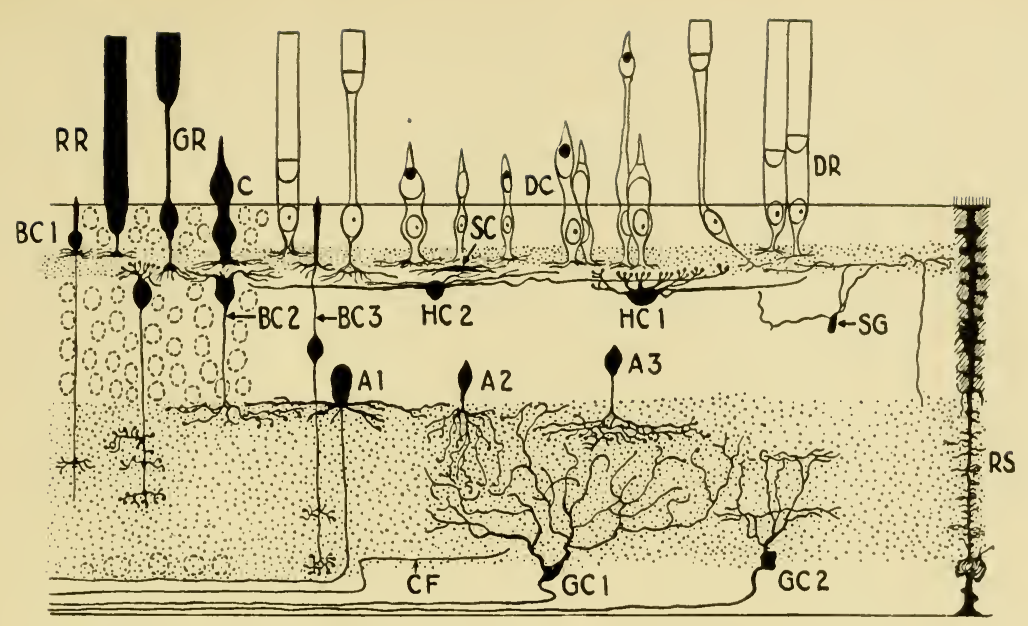

FIG. 19. Composite Golgi preparation of the amphibian retina. Redrawn and relabeled from Franz, 1913. RR, red rod; GR, green rod; C, cone; DC, double cone; DR, double rod; $\mathrm{BC} 1$, dispersed bipolar cell; SC, supporting cell; $\mathrm{HC} 1, \mathrm{HC} 2$, horizontal ganglion cells; $\mathrm{BC} 2, \mathrm{BC}$, bipolar cells; $\mathrm{SG}$, stellar ganglion cell; A1, "nervous" amacrine cell; A2, diffuse amacrine cell; A3, "layered" amacrine cell; GC1, GC2, ganglion cells; RS, radial supporting cell (Müller fiber); CF, centrifugal nerve fiber.

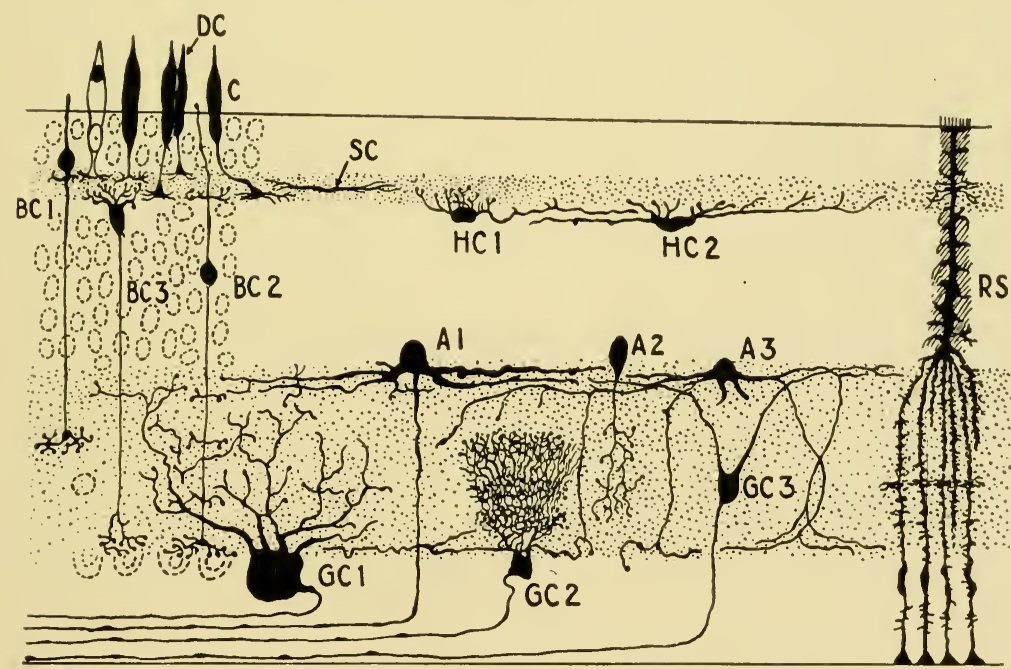

FIG. 20. Composite Golgi preparation of the reptilian retina. Redrawn and relabeled from Franz, 1913. C, cone; DC, double cone; SC, supporting cell; $\mathrm{HC} 1, \mathrm{HC} 2$, horizontal ganglion cells; $\mathrm{BC} 1$, dispersed bipolar cell; $\mathrm{BC} 2, \mathrm{BC} 3$, bipolar cells; A1, "nervous" amacrine cell; A2, diffuse amacrine cell; A3, "layered" amacrine cell; GC1, diffuse ganglion cell; GC2, "layered" ganglion cell; GC3, dispersed ganglion cell; RS, radial supporting cell. 


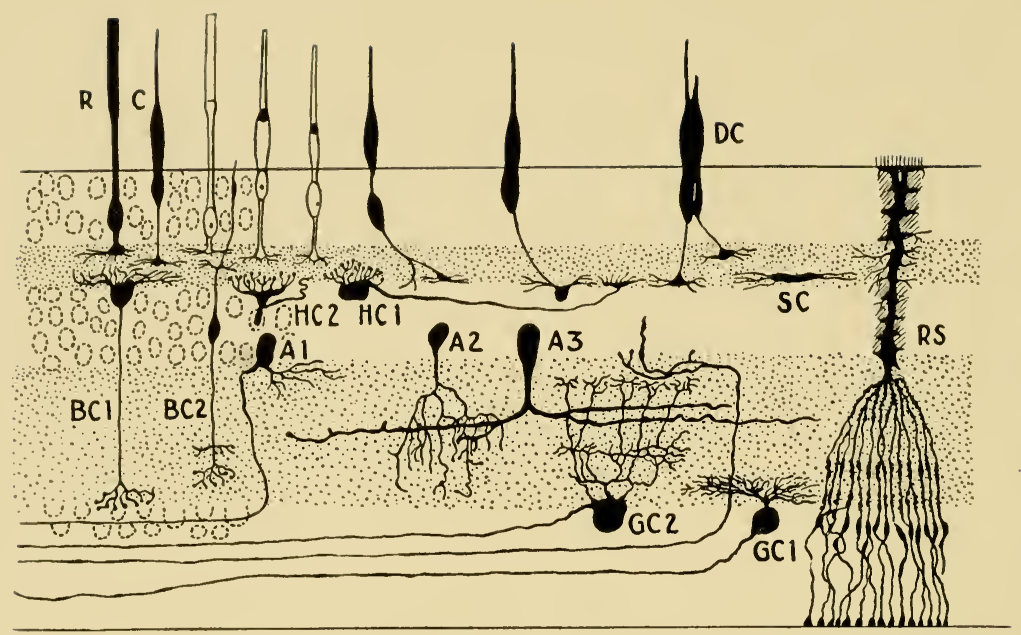

FIG. 21. Composite Golgi preparation of the avian retina. Redrawn and relabeled from Franz, 1913. R, rod; C, cone; DC, double cone; SC, supporting cell; $\mathrm{HC} 1, \mathrm{HC} 2$, horizontal ganglion cells; $\mathrm{BC} 1, \mathrm{BC} 2$, bipolar cells; $\mathrm{A} 1$, "nervous" amacrine cell; A2, diffuse amacrine cell; A3, "layered" amacrine cell; GC1, GC2, ganglion cells; RS, radial supporting cell (Müller fiber).

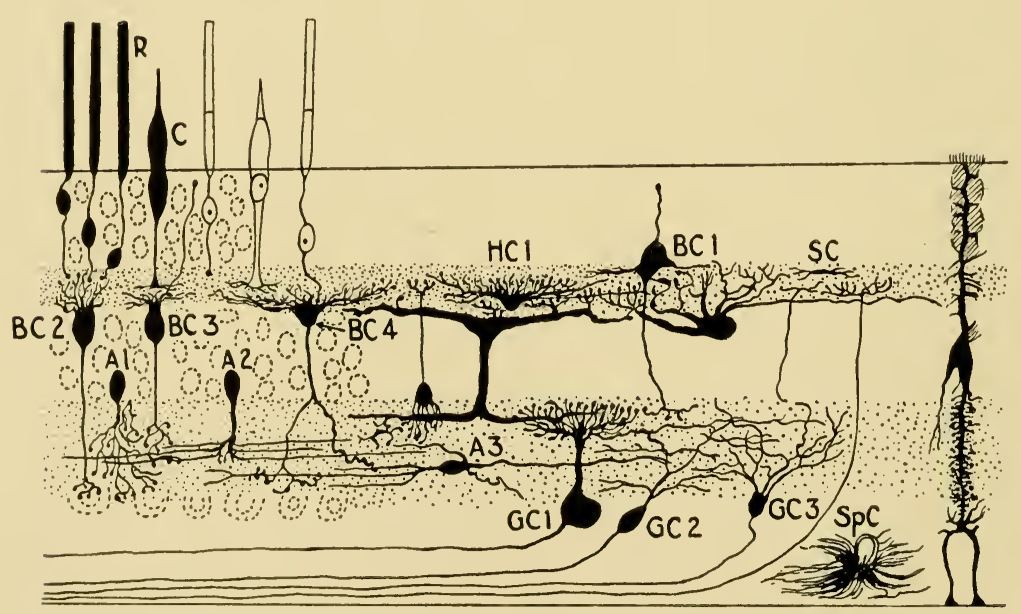

Fig. 22. Composite Golgi preparation of the mammalian retina. Redrawn and relabeled from Franz, 1913. R, rod; C, cone; SC, supporting cell; HC1$\mathrm{HC} 3$, horizontal ganglion cells; $\mathrm{BC} 1$, dispersed bipolar cell; $\mathrm{BC} 2$, rod bipolar cell; $\mathrm{BC} 3$, cone bipolar cell; BC4, "hoop" bipolar cell; A1, A2, amacrine cells; A3, dispersed amacrine cell; GC1, GC2, GC3, ganglion cells; RS, radial supporting cell; SPC, spider cell. 
1. The macula lutea measuring from 1 to $3 \mathrm{~mm}$. in diameter and subtending an angle of $4^{\circ}$ to $12^{\circ}$.

2. The rod-free area within the macula containing cones only.

3. The forea centralis (central pit) in the center.

The human fovea lies approximately $3.5 \mathrm{~mm}$. temporal to the optic disc and about $0.8 \mathrm{~mm}$. below the horizontal meridian. The rod-free area, said to be circular, measures from $0.4 \mathrm{~mm}$. to $0.8 \mathrm{~mm}$. in diameter. It subtends an angle of

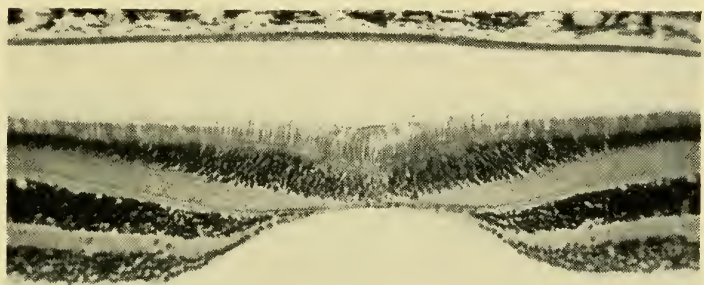

Fig. 23. Photomicrograph of the fovea centralis of the marmoset. $\times 100$.

approximately $3^{\circ}$. The human fovea is elliptical; the long horizontal axis measures $0.3 \mathrm{~mm}$. and the vertical axis $0.2 \mathrm{~mm}$., making an area of 0.5 to $0.6 \mathrm{sq}$. mm. In the parafoveal region the retina reaches its greatest thickness. DukeElder states that on the temporal side it measures $275 \mu$ to $410 \mu$ and on the nasal side $220 \mu$ to $350 \mu$. My own measurements (meridian unknown) showed a thickness of $390 \mu$ (Table 1). This is not as thick as the parafoveal region of the chameleon and the hawk which have the thickest retinas of all the forms measured in this study.

Apparently some authorities use the term "fovea" synonymously with the term " macula," giving to the fovea a diameter of 1 to $2 \mathrm{~mm}$. and calling the center the foveola.

There are so many points of interest centering about the structure, distribution, and function of the fovea in vertebrate eyes that a more detailed discussion will be resumed in a later chapter. 
The older concept of the architecture of the retinal transmitting apparatus is illustrated in its simplest form in Figure 24. Based upon the composite figures from Golgi preparations, the idea has been generally prevalent that the cone conducting system is discrete and separate from the rod conducting system. Each cone, in this scheme, has a separate, individual connection with a single bipolar and a

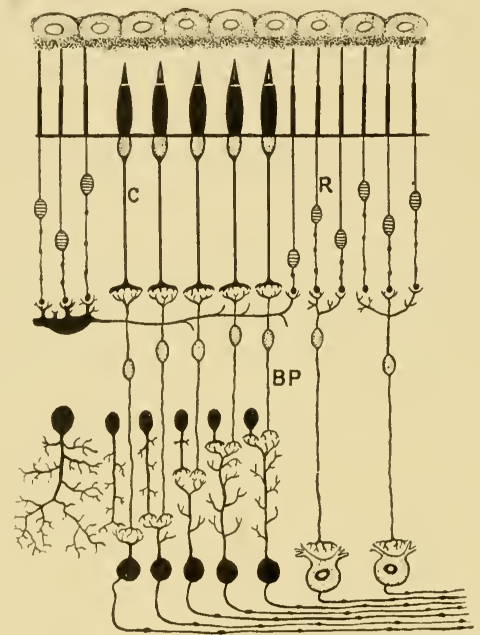

Fig. 24. Older concept of the connections of the retinal neurones, showing individual connection of the cones with a single bipolar cell, and multiple connections of the rods with a single bipolar cell. $\mathrm{C}$, cone; $\mathrm{R}$, rod; BP, bipolar cell. (Based upon Golgi preparations from Greeff and Cajal.)

ganglion cell, whereas many rods collect into a single bipolar and a single ganglion cell. This arrangement has been regarded as favoring summated conduction of the rods and isolated, individual conduction of the cones, thus not only enhancing rod vision at low illuminations, but favoring greater visual acuity of the cones at high intensities.

Whereas the above is true, in a way, especially as regards foveal cone conduction, it has been shown recently by Polyak (1941) that, in general, the situation is not nearly so simple, and that, outside the fovea, rod and cone conduction may involve the same transmitting and integrating neurones, thus producing mixed rod and cone systems. In this sense the 


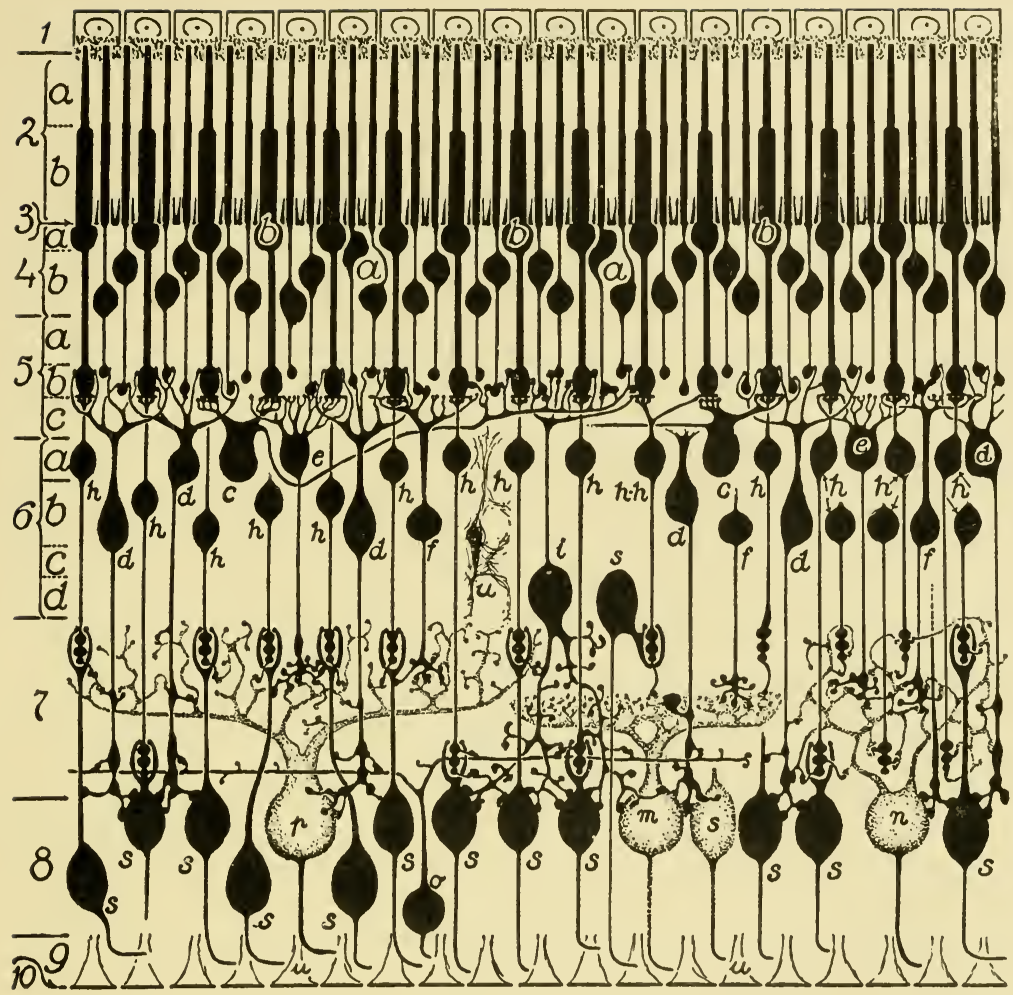

Fig. 25. Scheme of the structure of the primate retina as shown by Polyak, 1941, Chicago Iniversity Press. Iegends and designations are given in fontnote 1 .

two systems become intimately united into a single compact tissue pattern (Figures 25, 26, 27, 28). ${ }^{1}$

According to Polyak, the rod system is composed of three links as follows (Figure 27, A-C): the rods (a), all of the

${ }^{1}$ In order to facilitate the interpretation of Polyak's illustrations which are reproduced in Figures 25, 26, 27, 28 a list of his legends and their designations are given as follows:

a-rod

$\mathrm{b}$ - cone

c-horizontal cell

d-mop bipolar cell

e-brush bipolar cell

f-flat bipolar cell

$\mathrm{h}$ - midget bipolar cell

i-centrifugal bipolar (amacrine) cell $\mathrm{k}$ - amacrine cell

l-internal association cell (amacrine cell)

$\mathrm{m}$ - diffuse ganglion cell

$\mathrm{n}$ - shrub ganglion cell

$o$ - diffuse ganglion cell

$\mathrm{p}$-diffuse ganglion cell

$\mathrm{r}$ - diffuse ganglion cell

$\mathrm{s}$ - midget ganglion cell 
mop bipolars (d) and some of the brush and flat bipolars $(\mathrm{e}, \mathrm{f})$, and probably all varieties of ganglion cells $(\mathrm{m}, \mathrm{n}, \mathrm{o}$, $\mathrm{p}, \mathrm{r}, \mathrm{s})$. The rods are thus related to the ganglion cells by diffuse bipolars only. This system does not involve the midget bipolars (h) or association cells (c) or the amacrine

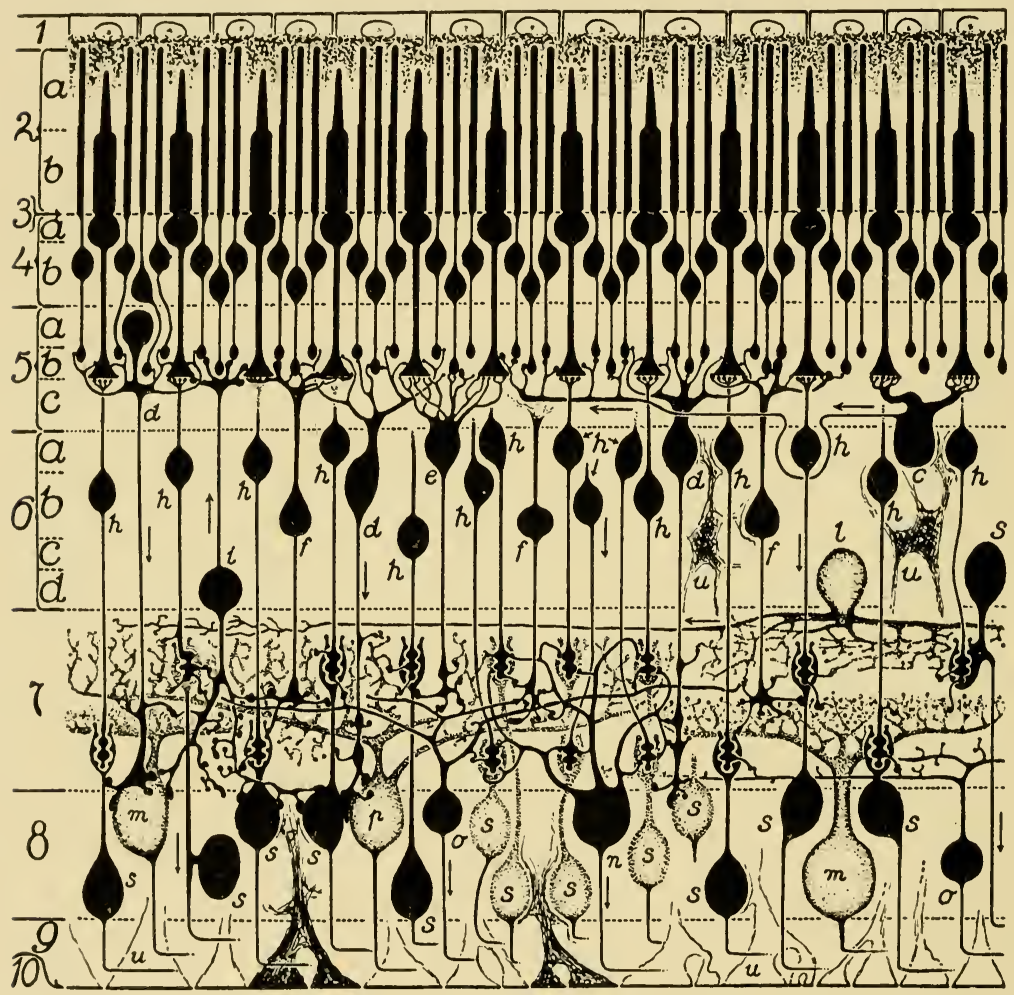

Frg. 26. Scheme of the primate retina, showing types of neurones and their synaptic relations as shown by Polyak, 1941, Chicago University Press.

cells (i, $k, 1)$. The rod system is thus thought of as made up of physiological units, each composed of a group of rods, of a fascicle of diffuse bipolar's, and a group of ganglion cells. These units are not completely separated, but overlap and merge throughout the retina.

In the cone system (Figure 27, D), the cones may synapse with all varieties of centripetal bipolars $(d, e, f, h)$, which in 
turn may connect with all varieties of ganglion cells. Thus the cone impulses may be transmitted to the ganglion cells by any or all of the above bipolars. As in the rod system, the cone functional units are not entirely separated from each
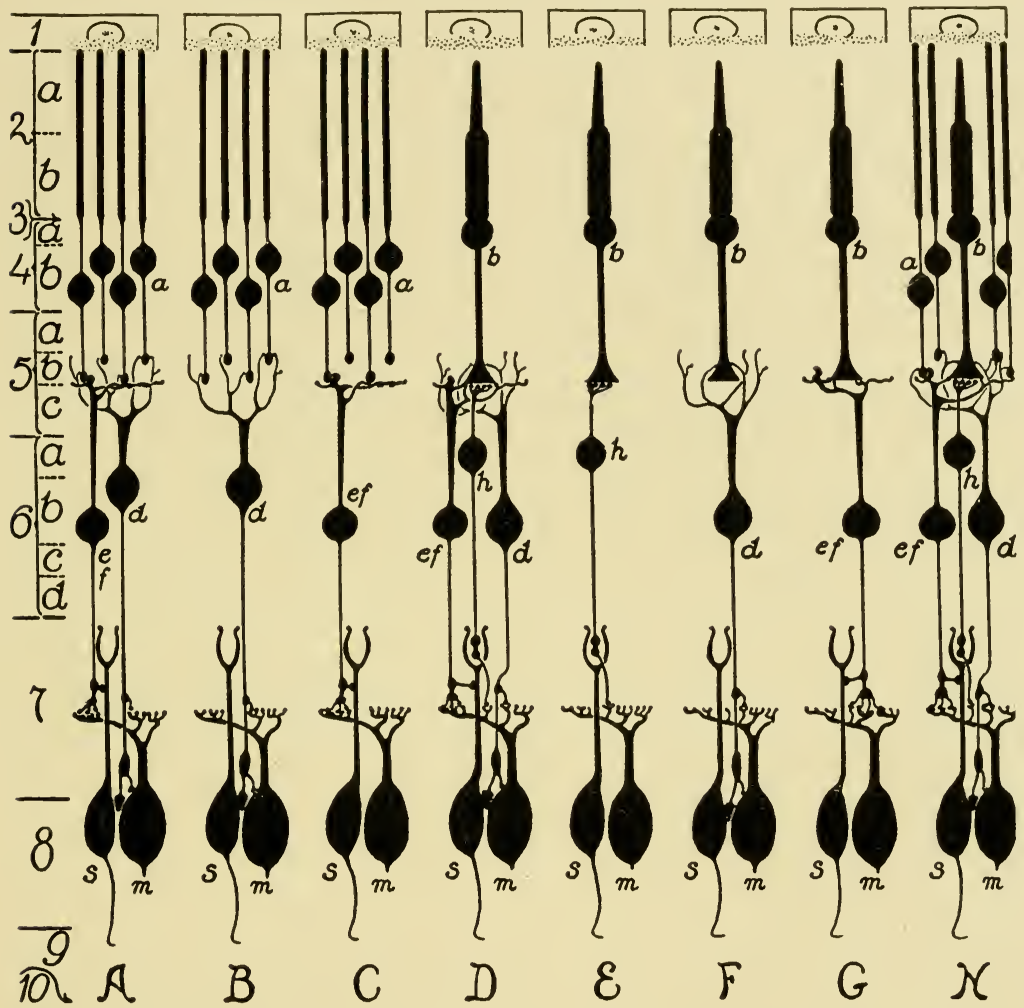

FIG. 27. The grouping of the primate retinal neurones into functional systems according to Polyak, 1941, Chicago University Press. A, rod system; $\mathrm{B}$, rod system with only one intermediate constituent (mop bipolar, $d$ ); $\mathrm{C}$, rod system with only the brush (e) or the flat bipolar (f) as the constituent; D, cone system; E, pure cone system with midget bipolar (h) as intermediate constituent; F, cone system with mop bipolar (d) as the intermediate constituent; $\mathrm{G}$, cone system with either the brush (e) or the flat bipolar (f) as the constituent; $\mathrm{H}$, mixed or common rod and cone system.

other because of the overlapping of diffuse bipolars and ganglion cells. Whereas this is the situation met with in the extra-foveal regions, Polyak's findings show that at the fovea one meets with what he designates "the pure, or 
private, cone system" (Figure 27, E). In this region there are no diffuse ganglion cells (indicated by $m$ in Figure 27) and the mop bipolars (d) are also lacking. Thus a cone impulse passes to a midget bipolar (h) and thence to a midget ganglion cell (s) without any lateral or transverse

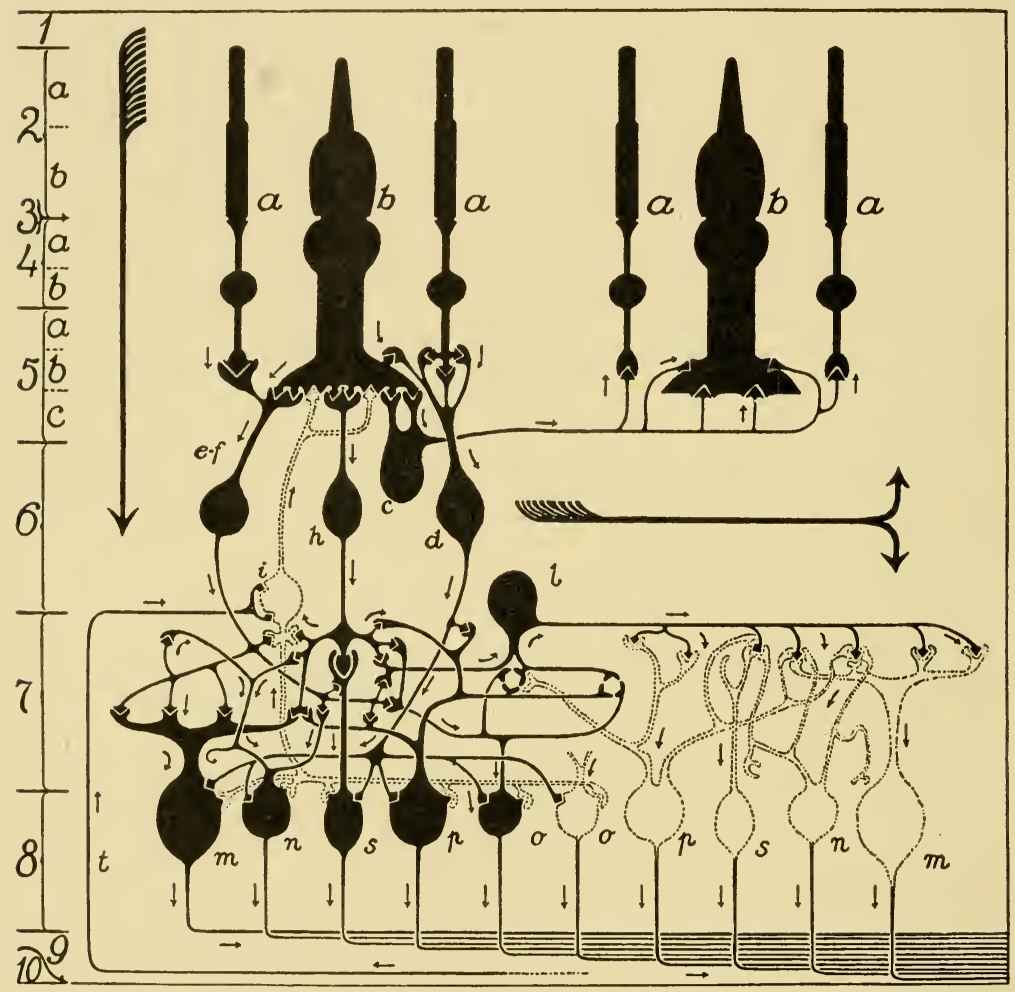

FIG. 28. The structure of the primate retina reduced to essentials to show the propagation of the retinal impulses from the photoreceptors to other parts of the retina, to the brain, and from the latter back to the retina. Direction of impulses indicated by arrows. (From Polyak, 1941, Chicago University Press.)

overlapping between the functional units. Thus at the fovea, the pure cone system is made up solely of monosynaptic structural-functional units which are arranged parallel to each other, so that impulses set up in individual cones are transmitted along discrete and private pathways without fusion or interference from adjacent parallel systems. This 
arrangement at the fovea (the pure, or private, cone system) is thus the instrument for the most delicate discrimination of visual stimuli.

In the mixed rod and cone system (Figure 27, H) there are three links as in the separate rod and separate cone system. These include: rods (a) and cones (b), all types of bipolars $(\mathrm{d}, \mathrm{e}, \mathrm{f}, \mathrm{h})$ and all ganglion cell types. In this functional system each diffuse bipolar is related to a closely packed group of both rods and cones. Such groups merge one with another, because of the overlapping of the bipolar and ganglion dendrites. The midget bipolars (concerned with pure cone conduction at the fovea) also form an integral part of this system. In connection with this mixed conduction system Polyak (p. 391) says: "The mixed, or common, rod and cone system apparently is active whenever the conditions of the stimulation and response are such as to affect simultaneously both the rods and cones. This is possibly realized during daylight, or diurnal or photopic, vision, or, at any rate, during the transition from the photopic to the scotopic vision, and vice versa. . . . In other words, under the conditions when both photoreceptors are activated, the diffuse bipolars transmit the impulses from both the rods and the cones, to which in this capacity they serve as a common channel - although even here there may be finer gradations or 'selecting' of certain kinds or shades of impulses from the common rod and cone excitations by the particular bipolar varieties."

The intraretinal association or integrating systems are represented by the horizontal cells (Figure 25, c), the centrifugal bipolars (Figure 25, i) and possibly by the amacrine cells (Figure 26, l). The centrifugal bipolar cell (i) with its dendrites in the internal molecular (plexiform) layer, apparently sends its axone terminals to the inner portions of the photoreceptors, and thus relays impulses in a centrifugal direction, i.e. opposite to those carried by the centripetal bipolars. It is suggested by Polyak that these centrifugal bipolar cells may serve for the transmission of influences 
from the brain to the photoreceptors. Since, however, they contact the centripetal bipolars $(\mathrm{d}, \mathrm{e}, \mathrm{f}, \mathrm{h})$ and the ganglion cells $(\mathrm{m}, \mathrm{n}, \mathrm{o}, \mathrm{p}, \mathrm{s})$ they may serve also as links along which the visual cells are subjected to the influence of cells lining the inner molecular layer, and thus to modified influences originating in the photoreceptors themselves. Thus the centrifugal bipolars (i) serve as units in a system composed of photoreceptors $(\mathrm{a}, \mathrm{b})$, centripetal bipolars (d, e, f, h), ganglion cells $(\mathrm{m}, \mathrm{n}, \mathrm{o}, \mathrm{p}, \mathrm{s})$, centrifugal bipolars (i) and photoreceptors. This assemblage resembles a reflex arc, and Polyak suggests that it may serve as a self-regulating intraretinal reflex mechanism whose probable function is to decrease or increase the photoreceptor thresholds, or to exert a modifying influence upon dynamic changes set up in the bipolars and ganglion cells by the photoreceptors.

A synopsis of the propagation of impulses through the retina (according to Polyak) is given in Figure 28. Impulses from the rods and cones ( $a$ and b) reach the horizontal cells (c) and are transmitted to surrounding rods and cones. The impulses discharge also into all varieties of bipolar cells (d, e, f, h) which serve as analyzers, from whence the impulses reach all varieties of ganglion cells for discharge into the optic fibers. Impulses from the bipolar cells and ganglion cells also discharge into the centrifugal bipolar cell (i) whence they are relayed back to the photoreceptors. Since this cell (i) also has connections with the efferent fibers (t), it serves as the main link in a conducting system whereby messages may pass from the brain to the photoreceptors. In this scheme the amacrine cell (l) supposedly intercepts impulses from the bipolar cells and spreads them to surrounding ganglion cells $(m, n, o, p, s)$.

Polyak's demonstration of the existence of both individual and mixed conduction systems for the rods and cones is a most valuable contribution, and one which the physiologist will be obliged to consider in his interpretations of any physiological data dealing with the major visual functions. 


\section{CHAPTER III}

\section{THE STRUCTURE OF VISUAL CELLS}

In 1866 Max Schultze made the fundamental discovery that the vertebrate retina, and particularly that of man, possesses two types of visual elements, namely, rods and cones; and that these elements subserve different visual functions. Further elaboration of his findings were made by Parinaud and by von Kries, and led respectively to the socalled Theory of the Double Retina of Parinaud and the

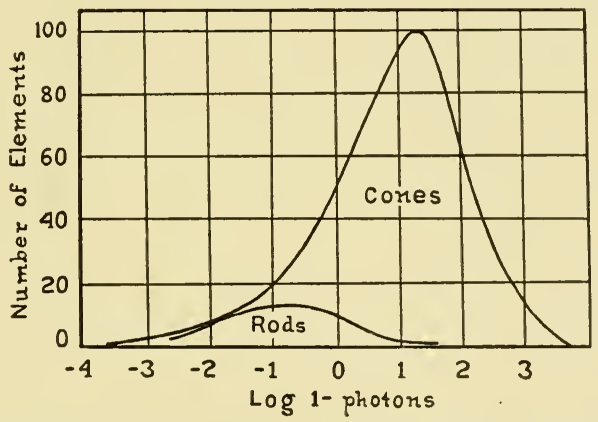

FIg. 29. Distribution of rod and cone thresholds, according to Hecht, 1928, Arch. Ophthal., v. 57.

Duplicity Theory of von Kries. ${ }^{1}$ The duplicity theory of vision, as we understand it, implies that the rods are concerned with colorless vision at low intensities of illumination (scotopic vision) and that the cones constitute the apparatus concerned with vision at high intensities of illumination and with color (photopic vision). The cones are not necessarily assumed to be utterly useless at night, but are relatively so because the majority of them possess thresholds which are much higher than the highest thresholds of the rods (Figure 29). Conversely the rods, which have low thresholds,

${ }^{1}$ Also referred to as the "Duplexity Theory," v. Crozier and Wolf. Proc. Nat. Acad. Sci., v. 24, p. 538, 1938. 
are apparently not entirely useless in daytime because some nocturnal animals with rods only can see in daytime. Typically, however, animals with pure rod retinas become more active with approaching dusk.

The duplicity theory, since its original conception by M. Schultze and its later elaboration, has been generally regarded as well substantiated. There are, however, a few workers who have taken exception to the validity of this theory. In this connection can be cited the work of Mademoiselle Verrier (1935) who regards the so-called rods and cones as representing extreme structural variations of a single photoreceptor cell - thus doing away with the concept of morphological and physiological duality in the retina. Regardless of this, the duplicity theory has received, in recent years, indubitable support from physiologists (see Hecht, 1937, 1938a; also Crozier et al. 1937-1941) in their quantitative physiological measurements of the major functions of vision, viz., dark adaptation, intensity discrimination, visual acuity, and the phenomenon of flicker. ${ }^{1}$ All one has to do is to consult the data and the curves dealing with

1 Crozier and Wolf ( $J$. Gen. Physiol., 1937 et seq.) have provided the most comprehensive body of data on the responses of lower vertebrates and invertebrates to flickered lights. A number of fishes, the newt Triturus, and the frog, all of which possess both rods and cones, yielded dual functions in these experiments such as are obtained characteristically from duplex retinas. As might be expected certain arthropods (Asellus, dragon fly larvae, bees, crayfish) yielded single functions. The same appeared to be true of the gecko, which possesses an all-rod retina; the turtle (Pseudemys scripta), the horned toad (Phrynosoma cornutum), and the zebra finch (Taeniopygia castanotis) which the authors report as possessing cones only. To the degree that the results from retinas containing one type of receptor were found to be simple, those from duplex retinas dual, these data appear to support the essential features of the duplicity (duplexity) theory. It was found, however, that the gecko and the turtle (Pseudemys) responded over an almost identical range of intensities intermediate between those characteristic of human rods and cones. These authors (op. cit.) point out, on this basis, that the general thesis that cones are less sensitive than rods falls through in this instance. It is probably true that this distinction, like any other single distinction between rods and cones, fails to divide them clearly in specific instances. Each of these receptors is characterized by a complex of properties, no one of which takes precedence over the other. It would seem, therefore, that this observation could hardly be interpreted as a basic difficulty for the duplexity theory. It might be of interest to note that Pseudemys (according to Walls, $1942 b$ ) is a turtle of relatively nocturnal habit, and that its retina contains considerable numbers of rods.

It may be pertinent to point out, in addition, that the intensity range over which any eye functions may be affected greatly by factors extrinsic to the photoreceptors themselves, e.g., size of pupil, or the presence of intraocular filters. 
the above listed functions to see and be convinced that rod function and cone function are two separate and discrete processes. It is evident, therefore, that we need not refer to the Duplicity Theory as theory but as fact, and that the retina must be regarded as a dual sense organ. In the light of these facts it becomes apparent that the presence and relative distribution of rods and cones in the same eye, as well as in different vertebrate eyes, becomes a matter of first importance to anyone who desires to study vision from a comparative point of view.

Although rods and cones each have specific characteristics, they are constructed upon a common general plan. Both possess an inner and an outer segment, a nucleus, and a centripetal fiber which brings them into relation with the bipolar transmitting cells. They extend through the pores of the fenestrated external limiting membrane so that their nuclei and centripetal fibers lie internal to it, whereas the main bulk of the cell lies external (Figure 24). In most animals with dual retinas the cone nuclei lie closer to the external limiting membrane than do the rod nuclei (e.g. mammals and most fishes). In amphibia, however, the condition is reversed, as it is also for the dual retinas of reptiles, and presumably for birds, although in this latter group the nuclei of the two types of visual cells are less distinguishable one from the other.

Cones. Typically the cone is a conical-shaped element possessing an inner and an outer segment. It is the conical shape of the outer segment which is the morphological feature used to distinguish this cell as a cone, whereas the shape of the outer segment of the rod is typically cylindrical. There are marked deviations from the typical condition and cones, as well as rods, vary considerably in form, size, and structure. In the region of the fovea, the cones are long, slender attenuated structures which look more like rods, yet their function fits in with the facts of cone physiology. In the human fovea they are said to measure about $85 \mu$ in length according to Greeff (1899), from 58 to $67 \mu$ according 
to Salzmann (1912), and $70 \mu$ according to Polyak (1941). The foveal cones of the chameleon have been found to be longer than human foveal cones (Table 1). They are also longer in the marmoset. The diameter of the human foveal cone is said to range from $1.5 \mu$ to $3 \mu$. The usually accepted diameter is approximately $2.5 \mu$. According to Polyak, in the center of the outer fovea, the cone inner segment is $1.5 \mu$ thick; the outer segment $1 \mu$. In the slope of the outer fovea the inner segments are $2.3 \mu$; the outer segments $1.3 \mu$. In the edge of the outer fovea the inner segments are $2.5 \mu$ in diameter; the outer segments approximately $1.3 \mu$. He says also that in the center of the fovea the dead interval between two adjacent cones is $0.3 \mu$, whereas from $100 \mu$ to $150 \mu$ outside the center the dead interval is $0.5 \mu$ (v. Polyak, op. cit., Figure 38). Some have estimated that the distance between two adjacent cones is $2.5 \mu$. As regards the number of cones in the rod-free region of the macula, various estimates have been given. Duke-Elder states (p. 96) that there are from 13,000 to 14,000 per $0.1 \mathrm{sq} . \mathrm{mm}$. in the region of the macula, which would mean 130,000 to 140,000 per sq. mm. However, on page 91, he says there are 13,000 cones in the region of the macula and 4000 at the region of the fovea. These estimates seem not to agree. According to Osterberg (cited from Wolff, 1940) there are 147,300 cones per sq. mm. at the fovea. At a point where the rods begin $(130 \mu$ from the center of the fovea) there are 74,800 cones per sq. mm.; $3 \mathrm{~mm}$. farther there are 6000 per sq. mm., and $10 \mathrm{~mm}$. from the fovea they number about 4000. Polyak has estimated that, in the center of the fovea, where all the inner nuclear layers and blood vessels are practically absent (the territory coextensive with the foveola), there are 25,000 cones. He says also that the human central territory, where rods are absent, measures approximately $500 \mu$ across $\left(1^{\circ} 40^{\prime}\right.$, or $50^{\prime}$ of arc on both sides of the ideal fixation-point). 'The number of cones computed in this region is given as approximately 34,000. This would mean approximately 136,000 cones per sq. mm., and would be of similar order of magni- 
tude as given by Duke-Elder (130,000-140,000) and Osterberg $(147,000)$. From this it may be safe to say that, in general, there are something of the order of 140,000 cones per sq. mm. at the macula, and that the cones in the human

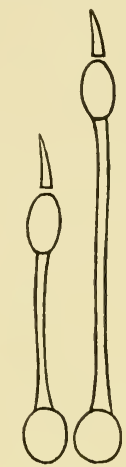

A

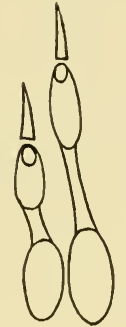

$\mathrm{B}$

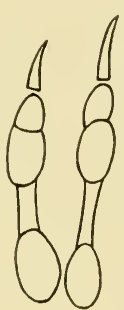

$\mathrm{C}$

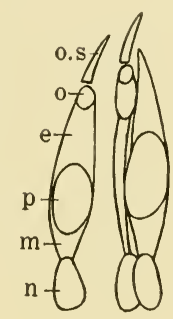

D

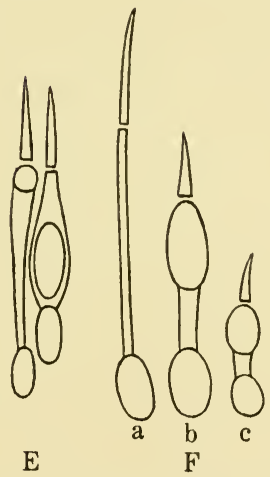

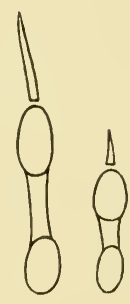

G

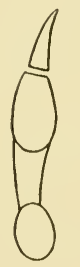

$\mathrm{H}$

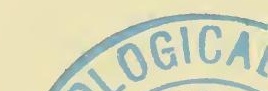

FIG. 30. Cones from various vertebrates. A, catfish; B, frog; C, alligator; D, lizard; E, chicken; F, monkey (marmoset); G, rhesus monkey; H, man. (Figures are not drawn to same scale.) a, foveal cone; b, parafoveal cone; c, peripheral cone; $\mathrm{n}$, nucleus; $\mathrm{m}$, myoid; $\mathrm{p}$, paraboloid; e, ellipsoid; o, oil globule; o.s., outer segment. (Figures of visual cells of representative vertebrates drawn to a uniform magnification are given by Arey, 1928, Special Cytology, v. 2, Paul B. Hoeber. New York.)

eye total about 7,000,000, whereas the rods have been estimated as from $75,000,000$ to $170,000,000$. As one approaches the ora serrata, the cones become gradually shorter and thicker and also less numerous per unit area. This in general is true of all animals. 
Typically the inner segment of the cone, which is somewhat barrel- or keg-shaped, possesses an ellipsoid and a myoid element. Frequently in addition, there is present a highly refractive globular structure called the paraboloid, and in some classes an oil globule (Figure 30). The myoid element in some forms (fishes, amphibians, birds) is contractile; hence its shape and length varies. It contracts in the light and lengthens in the dark. According to Arey $(1915,1916 a)$ the maximum ratio of an extended to a contracted myoid in fishes may be $10: 1$. The ellipsoid or lentiform body lies next to the external segment (except in cones with oil globules). It too varies somewhat in shape and size. In mammals it occupies two-thirds of the volume of the inner segment. It is said to be absent in some reptiles. A paraboloid is found in some amphibians, reptiles, and in the accessory component of double cones

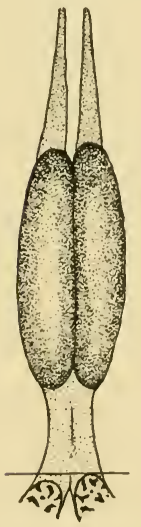

FIG. 31. Twin cone of the perch. $\times 1000$. (Redrawn from Arey, 1928, Special Cytology, v. 2, Paul B. Hoeber, New York.) (Figure 30, D and E). It is absent in the cones of teleost fishes, mammals, anthropoids, and man.

Double cones (Figure 30, D) occur in all retinas except in mammals. They consist of two components or members (a principal and an accessory part). The principal (chief or far) cone has a long narrow myoid, a long ellipsoid, and an oil globule, there being no paraboloid. The accessory (or near) cone, which is much broader and shorter than the principal cone, has a typically short myoid, a paraboloid, a granular ellipsoid, but no oil drop. In addition to double cones, there occurs also the so-called twin cones in which each member is of equal size (Figure 31). In fishes they are said to occur in forms with well developed eyes and never in nocturnal fishes with small eyes. According to Wunder (1926) this makes for better vision. The double and twin cones presumably arise from the fusion of pre-existing single cones. Levi (1901), however, 
is of the opinion that they are incomplete division forms of a single embryonic cell.

The oil droplets are present in amphibians, reptiles, and birds, but are lacking in fishes and mammals. When present they lie at the distal end of the inner segment (Figure 30). In reptiles and birds they are colored. Whereas all colors are claimed to be represented, those towards the blue-violet end of the spectrum are rare. They are most striking in birds where Waelchli (1883) distinguished some eight varieties. According to Wald (1939b), in the chicken they are red, golden, and greenish yellow in color, forming a color filter arrangement similar to that employed in many systems of color photography. In birds also certain regional distribution of colors may be made out, designated as the red field or the yellow field.

Rods. As with the cones, visual rods vary greatly in size and general proportions in different animals. In the amphibians, for example, the urodele rods are short, cylindrical structures; the anuran rods are much longer. They are long in fishes (light-adapted), birds, and man. The owl has notably long rods $(85 \mu)$. In the human they are said to have a length of $60 \mu$ in the fundus, and $40 \mu$ at the ora serrata. In many nocturnal forms they are actually filamentous (e.g. rodents, lemurs). In Nyctipithecus (owl monkey) which lacks cones, the rods are also very slender and closely packed (Figure 54). However, in the bat the rods are quite short $(26 \mu)$, and much less filamentous (Detwiler, 1924, Figure 1). Rods, like cones, possess an inner and an outer segment, between which there exists in many forms a refractive disc or intermediate plate. The inner segment consists of a myoid and an ellipsoid. The myoid of teleost fishes, anuran amphibians, and birds is contractile, shortening in darkness and elongating in the light. Measurements of the rods of Ameiurus have shown a length of $92-93 \mu$ in light and $33.8 \mu$ in darkness. The difference between the two conditions is shown in Figures 57 and 58. The outer segment, which is strongly refractive, tends to stain specifically with acid 
dyes; the inner segment shows preference for basic dyes. The staining reaction of the outer segment varies with the conditions of illumination.

Rods possess an ellipsoid or lentiform body at the distal end of the inner segment. In fishes and anuran amphibians

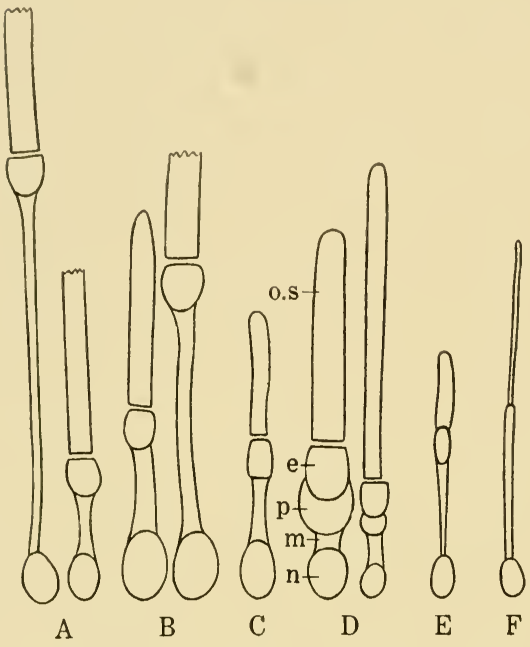

FIg. 32. Rods from various vertebrates. A, catfish; B, frog; C, alligator; D, nocturnal lizard (Gecko); E, chicken; $\mathrm{F}$, monkey; $\mathrm{n}$, nucleus; $\mathrm{m}$, myoid; p, paraboloid; e, ellipsoid; o.s., outer segment. (Figures are not drawn to same scale.) it is a single plano-convex element, truncated on its distal surface (v. Arey, 1928, plate 1). Most rods lack a paraboloid. This structure, however, is present in the rods of salamanders, turtles, Sphenodon (Walls, 1934a), and in the geckos (Detwiler, $1923 c$ ) where it is a very prominent barrel-shaped structure and much larger than the ellipsoid (Figure 32, D). Arey (op. cit.), in discussing the rods says (p. 893), "Lentiform bodies may well play a dioptric rôle. . . Many of their properties suggest this, and the ellipsoid-paraboloid combination is most interesting from such a viewpoint. Its components have geometrical forms like those of the component lenses in an achromatic objective, and both are more refractive than the surrounding medium. . . ."

Apparently the single or compound lentiform apparatus may serve to concentrate light upon the outer segments and perhaps cause it to diverge as it passes the focal plane. The colorless oil droplets in the cones may act in a similar way.

Rods are present in all vertebrates except the diurnal lizards. In diurnal turtles they are generally regarded as being absent, although Walls (1934a) claims that rods are 
present in small numbers. Their numbers vary greatly in different portions of the retina. In dual retinas they are typically less numerous in the fundus and more numerous peripherally. Thus the rod-cone ratio varies in different portions of the retina. Rods are entirely lacking in the region of the fovea.

\section{The Basis for Rod and Cone Classification}

The question as to what constitutes a rod or a cone would seem to be a relatively simple matter, but the great variety in the form of these cells in different animals makes a general classification difficult. Structural differentiation is based usually upon the form of the outer segment (coneshape vs. rod-shape). However, this does not meet all the requirements for, as pointed out above, the cones at the fovea are long cylindrical structures and look more like rods.

Pütter (1912) stressed the importance of the mode of connection with the bipolar cell as a real basis for classification. According to him cones have centripetal dendritic endings whereas rods have knob-like or molecular endings, regardless of their external form. This is the condition usually depicted in textbooks, which, of course, is correct for the mammalian and human eye, as well as for the eyes of teleost fishes. However, as will be seen in Figures 17 to 22, the rods of selachians, amphibians, reptiles, and diurnal birds (according to Franz) also possess dendritic endings. When we find visual cells, which from their general form (outer and inner segments) should be called rods, possessing typical cone connections (e.g. frogs, diurnal birds) there seems to be no reason for calling them cones because they terminate in dendrites. There is considerable reason to designate them as rods on functional as well as on structural grounds.

Diurnal lizards have cones only, but in the crocodilia, for example, both rods and cones occur, structurally as well as functionally. According to Pütter, they would all have to be regarded as cones because their centripetal terminations are similar to those found in the cones of man. 
Designating a visual cell as a rod or a cone on morphological grounds alone does not seem entirely useless. Anyone who has made an extensive histological study of the vertebrate retina soon becomes convinced of the existence of two different cellular types. However, there are other criteria which one can use if one is not entirely convinced by morphological evidence alone. Those who have had long experience with different staining methods are able to distinguish these two types of visual cells by their different chemical affinities.

Most of the work on differential staining of the visual cells has been done by Kolmer (1936). With Unna's orceinpolychrome methylene blue-tannin stain, he colored the outer segments of the cones deep blue, whereas the outer limbs of the rods remained unstained - indicating a physicochemical difference. With Mallory's stain, a distinct difference was seen in the cone and rod nuclei; the cone nuclei stained red with fuchsin, the rod nuclei stained orange. After fixation in chrome-containing fluids and treatment with nascent chlorine, Unna's stain colored the inner and outer portions of the cones a deep blue (with Wasserblau); the corresponding portions of the rods were colored red (with safranin). These and still other methods demonstrated, in many vertebrates, a distinct difference between rods and cones. Wolff (1940), from whom the above information was taken, also states that he himself succeeded, in the human, in coloring the inner portions of the cones red with Mallory's triple stain after Zenker fixation, whereas the corresponding portion of the rod stained blue. This is of especial interest, for he found that the inner limbs of the rod-like cones stained as cones (red) and not as rods.

It is apparent, as more work is being done on the photochemistry of the retina, that the photopigments in the two types of cells are different. This also constitutes a strong argument in favor of the separateness of the visual elements.

Lastly, one must not fail to offer as additional evidence for the discreteness of visual cells the inverse photomechanical reactions which the cone and rod myoids exhibit in many 
vertebrates. When light plays upon the retina of fishes, amphibians, some reptiles and birds, the rod myoid elongates and the cone myoid contracts. In darkness the opposite response occurs. Since these phototropic reactions are directly opposite in character to the same stimulus, it is obvious that the rods and cones represent two different and distinct elements. The existence of intermediate forms seems very doubtful (Osawa, 1898). 


\section{CHAPTER IV}

\section{DIFFERENTIATION OF RETINAL ELEMENTS}

The general facts regarding the origin of the optic vesicle from the forebrain and its subsequent invagination to form the optic cup are well known. The story is adequately treated in most textbooks of embryology. The fundamental principles involved are common to all classes of vertebrates. In all forms, the outer layer of the optic cup remains largely undifferentiated and becomes the epithelial pigment layer. The inner (invaginated) layer becomes highly differentiated and specialized to form the elements of the retina proper. The anterior portions of both layers develop into the ectodermal part of the ciliary body and iris (pars ciliaris retinae and pars iridica retinae).

In the human eye the differentiation of the retina, according to Mann (1928), can be divided into three arbitrary stages as shown in the accompanying chart, modified slightly from Duke-Elder (1939).

During the first stage (fourth and fifth week of development), the wall of the vesicle is like that of the rest of the central nervous system in that it is divisible into two zones: 1) the primitive layer of neuro-epithelium containing 8 or 9 rows of oval nuclei, and 2) the marginal zone which is devoid of nuclei (Figure 33, A). The surface which borders the vesicle is covered by many fine cilia like those which persist in the central canal of the spinal cord. These cilia are of interest because of the contention by some embryologists that they eventually form the outer segments of the visual cells (Mann, 1928, p. 94; Arey, 1928, p. 890).

In embryos of $10 \mathrm{~mm}$., differentiation into layers becomes evident. The cells near the inner surface proliferate and migrate outward, a process which occurs most rapidly in the 
region of the posterior pole. The migration of cells from the primitive nuclear layer into the marginal layer produces an inner cell layer (Figure 33, B). Thus the original layer of cells becomes divided into an inner and an outer neuroblastic

1st STAGE

2nd STAGE

3rd STAGE

$\underline{\text { ADULT }}$

4-5 week

6th week -3rd month 3 rd -7 th month

Surface of marginal

layer

Internal limiting membrane

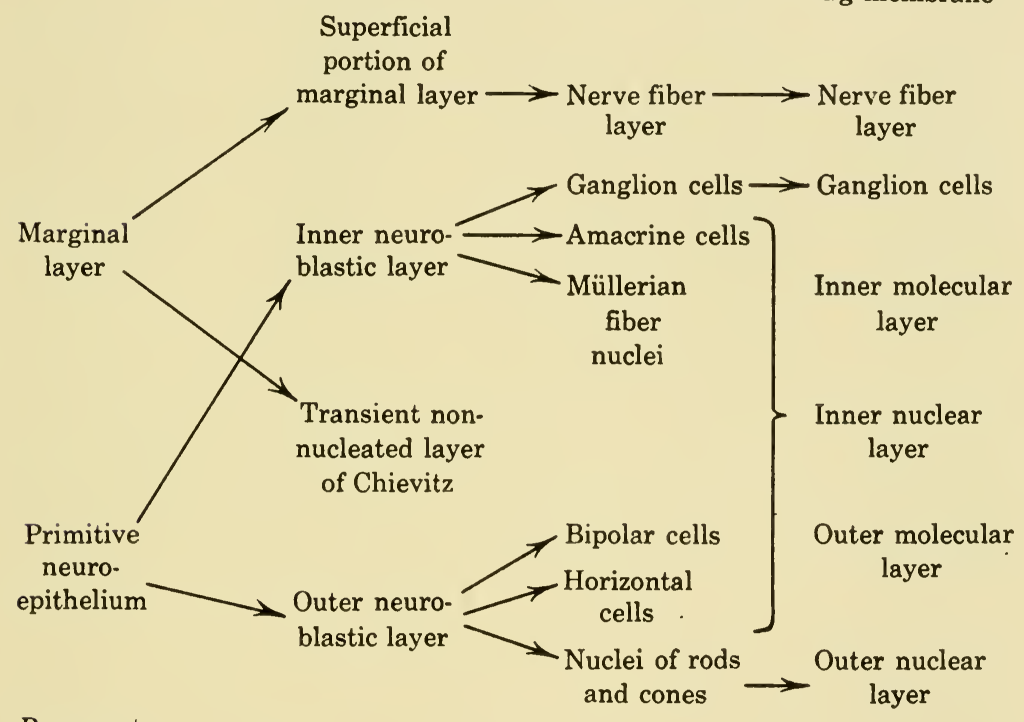

Superficial

portion of

Basement

membrane

External limit. ing membrane

Cilia Cilia Primitive rods and cones $\longrightarrow$ Rods and cones

Outer wall of optic cup Pigment epithelium

layer with an intervening narrow zone devoid of nuclei (transient non-nuclear layer of Chievitz). From these two neuroblastic layers the retina is constructed by further proliferation and differentiation, which proceeds from within outward. The ganglion cells are the first of the retinal elements to appear, the rods and cones the last. 
In the third stage the innermost cells of the inner neuroblastic layer begin to show characteristics of ganglion cells, sending out their axones centripetally into the optic stalk (Figure 33, C). The remainder of these cells become the

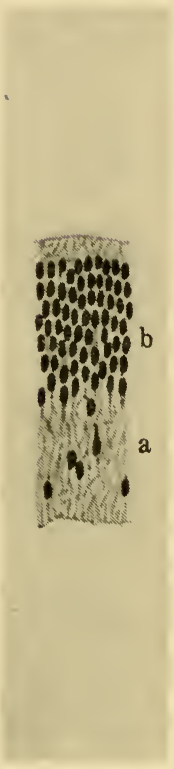

A

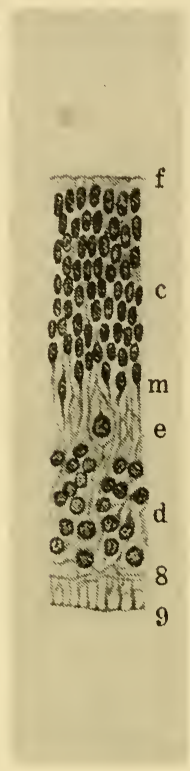

B

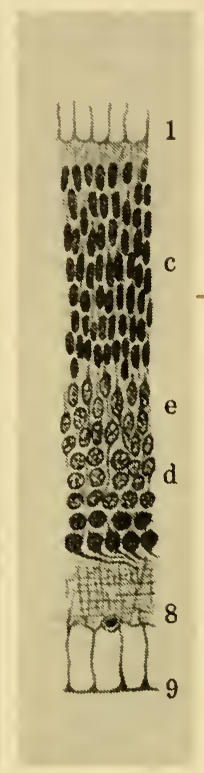

C

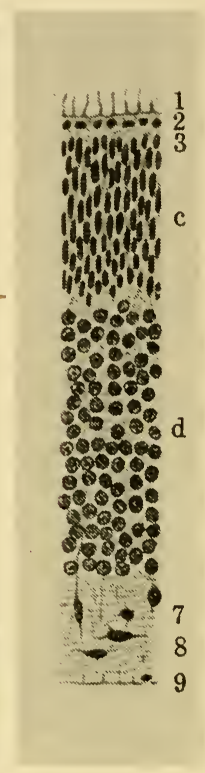

$\mathrm{D}$

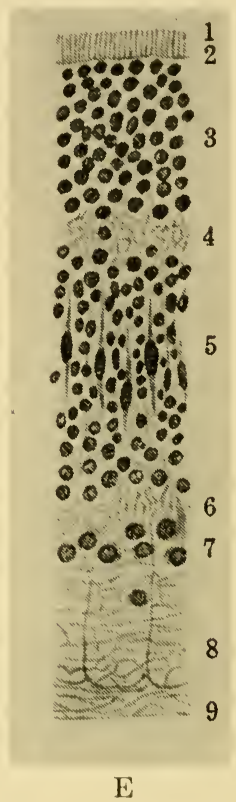

$\mathrm{E}$

Fig. 33. The development of the human retina. (Modified from DukeElder, 1939, C. V. Mosby Co., St. Louis.) Sections through the inner layer of the developing optic cup of A, a $12 \mathrm{~mm}$. embryo; B, a $17 \mathrm{~mm}$. embryo; C, a $21 \mathrm{~mm}$. embryo; D, a $48 \mathrm{~mm}$. embryo; and E, a $170 \mathrm{~mm}$. foetus. a, marginal layer; b, primitive neuroepithelium; c, outer neuroblastic layer; $d$, inner neuroblastic layer; e, transitory layer of Chievitz; f, basement membrane; $\mathrm{m}$, fibers of Müller. 1, rods and cones; 2, external limiting membrane; 3, nuclei of rods and cones; 4, outer molecular layer; 5 , inner granular layer; 6 , inner molecular layer; 7 , ganglion cells; 8 , nerve fiber layer; 9 , internal limiting membrane.

amacrine cells and the Müllerian cells, of which the latter send their processes to the inner surface, spread out, and unite with each other to form the internal limiting membrane.

The cells of the outer neuroblastic layer differentiate later than do those of the inner layer. The innermost ones become the bipolar cells, those more external, the horizontal cells, and the outermost become the nuclei of the rods and cones. 
A separation between the horizontal cells and the nuclei of the visual cells produces the external molecular layer. Finally the transient layer of Chievitz disappears. The amacrine cells and the nuclei of the Müller fibers (both of inner neuroblastic origin) coalesce with the bipolar and horizontal cells (of the outer neuroblastic layer) and collectively form the inner nuclear layer (Figure 33, E).

The rods and cones appear as protoplasmic extensions in the region of the external limiting membrane. The outermost boundaries of these cells form what might be termed the embryonic external limiting membrane. The definitive external limiting membrane is formed later by the fibers of Müller which extend to the outermost boundary of the rod and cone cells, where they form a membrane similar to the internal limiting membrane, but fenestrated. That the external limiting membrane extends out over the inner segments of the rods and cones is unlikely for, as Mann (1928) has pointed out, they appear before the growth of the outer processes of the Müllerian fibers. Their covering, therefore, must come from the original cell boundaries.

As regards the origin of the outer segments, Mann (op. cit.), although noncommittal, apparently favors the view that they originate from or can be identified with the cilia which originally lined the cavity of the central nervous system. In this connection she says (p. 94): "At $21 \mathrm{~mm}$., although cilia can be seen in the cavity of the fore-brain, the processes from the retina are definitely longer and more widely separated than before and can be looked on as beginnings of the outer limbs of the rods and cones. They thus would seem to arise by increase in size and reduction in number of preexisting cilia. Whether or not this is the case is difficult to determine since the structures involved are so delicate and so liable to give rise to artefacts during preparation. It is, however, worthy of note that while an appearance of cilia can be seen continuously on the inner wall of the optic cup, the outer wall cells very definitely lose them soon after the development of the pigment granules. One is therefore 
safe in drawing at least a close parallel if not a definite derivative relation between cilia and the primitive outer limbs of the visual elements."

A similar view apparently is held by Duke-Elder. With respect to this matter, Arey (1928) says, "An interpretation

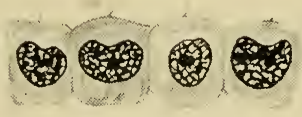

A

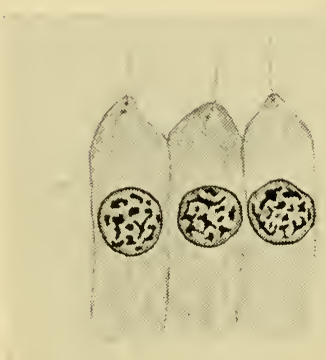

C

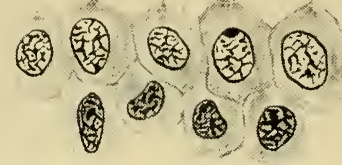

$\mathrm{B}$

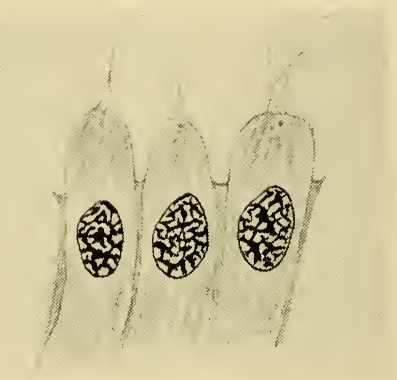

D

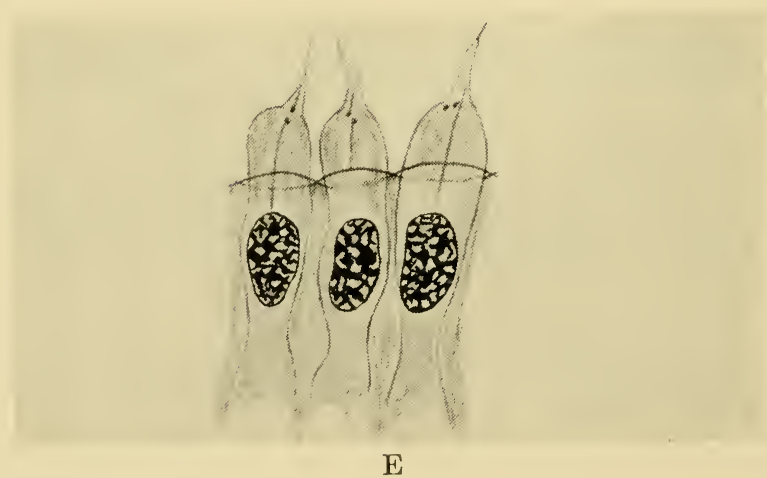

FIG. 34. Stages in the development of the human retinal cones. (Redrawn from Ida Mann, 1928, Cambridge University Press.) A, cone cells from fundus of a $48 \mathrm{~mm}$. embryo; B, cone cells from an $80 \mathrm{~mm}$. embryo; C and D, further stages of differentiation; $\mathrm{E}$, cones from the retina of an 8 month old foetus. 
of the rods and cones as robust cilia, homologous with those of the central canal of the embryonic neural tube, likewise does not appeal."

According to Mann the visual cones develop as shown in Figure 34. $A$ in this figure shows the condition at the posterior pole in a $48 \mathrm{~mm}$. embryo. Each cell has a very slight protoplasmic extension which indicates the origin of the inner segment. Each contains a very fine filament attached to the blunt apex of the cell and which lies within the cavity of the primary vesicle. This filament, according to her, represents the earliest recognizable outer limb of the cone, and shows the same relation and structure as did the cilium of the basement membrane in the earlier stages. $B$ shows the conditions in an $80 \mathrm{~mm}$. foetus. The nuclei have become spherical, the protoplasmic protrusions are longer and each contains a pair of darkly staining particles, the diplosome. The outer segment, which is more marked, appears to be attached in the region of the diplosome. According to Leboucq (1909), Magitot (1910), and Seefelder (1910), both rods and cones in the human retina develop simultaneously, and are dis-

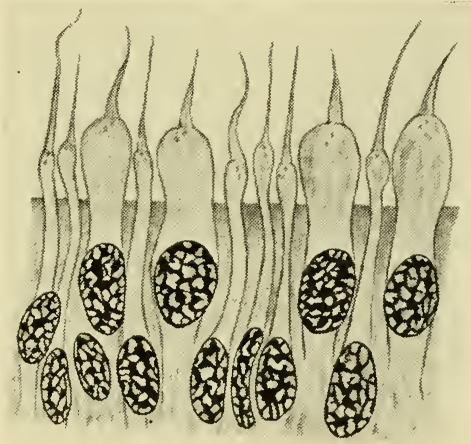

FIG. 35. Section showing rods and cones at the end of the seventh month. (Redrawn and modified from Mann, 1928, Cambridge University Press.) tinguishable mainly by the fact that in the cone the axis of the diplosome (centrosomes) is perpendicular to the long axis of the cell, whereas in the rod it is parallel to the long axis of the cell. This is not entirely borne out in the illustration which is reproduced in Figure 35. $C$ and $D$ (Figure 34) show further development of the cones and $E$ shows the condition of young cones during the eighth month. At this time the outer processes of the Müllerian fibers have reached the basement membrane and have united with one another 
around the bases of the cones to form the definitive external limiting membrane, through the pores of which the visual cells protrude. The outer segment appears to be connected with the diplosome and with the nucleus by a very fine filament.

The rods apparently have a similar mode of origin, but from the beginning are smaller and thinner and their nuclei occupy a deeper position (Figure 35 ).

Apparently the human retina does not reach its full differentiation until after birth. Particularly is this true of the macular region. Whereas the eye is said to be light sensitive at the seventh month, form perception and color discrimination are not acquired until after birth, and according to Mann (op. cit.) the macular region (with the fovea), which begins to differentiate late in foetal life (seven to eight months), continues to differentiate until about sixteen weeks post partem.

Many observers have claimed that the rods and cones are, from the beginning, discrete elements, and develop practically simultaneously (Levi, 1901, 1914; Leboucq, 1909; Magitot, 1910; Seefelder, 1910; Cajal, 1911; Detwiler and Laurens, 1921). Others have regarded the cones as specialized rods - viewing the rod as the more primitive element (Parsons, 1915; Kerr, 1919). Still others have concluded that cones represent early stages in the formation of rods (Bernard, 1903; Cameron, 1905, 1911). These last investigators came to this conclusion because they saw, in the early stages of the developing amphibian retina, conicalshaped elements only, whereas in later stages typical rods were prevalent. It is true that in the early stages of development of the amphibian retina, all the visual elements are cone-like in shape. This appearance, however, is not sufficient evidence to conclude that they are cones. In our own observations (Detwiler and Laurens, op. cit.) we showed that many of the so-called cones are really developing rods as evidenced by their larger size, the different position of their nuclei, and the lamellar arrangement of 
the materials in their outer segments (Figure 36, D). It was shown also (Detwiler, 1923b) that in Amblystoma larvae just prior to the feeding stages (Harrison's stage 44), the two categories of conical-shaped elements could be distinguished on physiological grounds as well. When such larvae

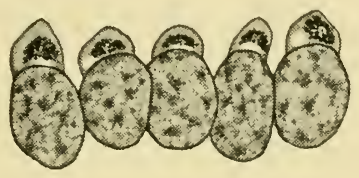

A

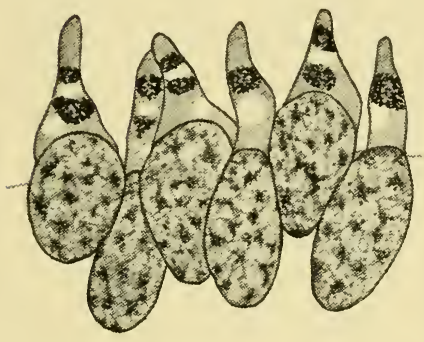

C

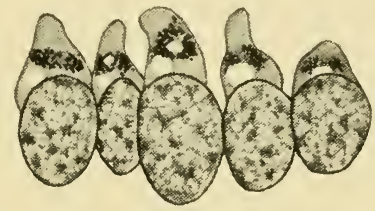

B

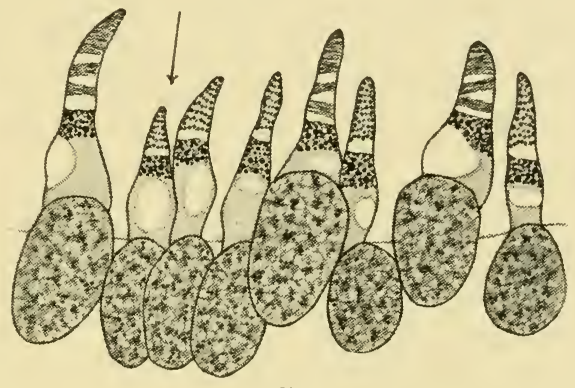

$\mathrm{D}$

Fig. 36. Stages in the early development of the visual cells in Amblystoma punctatum. A, retina of an embryo 11 days after tail-bud stage; B, 13 days after tail-bud stage; C, 15 days after tail-bud stage; D, 17 days after tail-bud stage. Arrow indicates double cone. $\times 920$. (From Detwiler and Laurens, 1921, J. Comp. Neur., v. 33.)

were light-adapted, the myoids of the true cones contracted, and when dark-adapted they elongated. The 'potential' conical-shaped rods, however, showed no phototropic response - a condition characteristic of the adult urodele rod (Figure 38). We concluded, therefore, on structural as well as on functional grounds that rods do not represent later stages in the formation of cones, neither is there any evidence to suggest that cones represent specialized rods, but that both are formed by divergent differentiation of a cell of low morphological specialization. These conclusions are in agreement with those of Levi $(1901,1914)$ who studied the development of the retina in Salamandra. 
The first stage in the development of the amphibian visual cell is the production of a protoplasmic bud and a clear achromatic globule from the cells of the external nuclear layer. The globule, which becomes the paraboloid

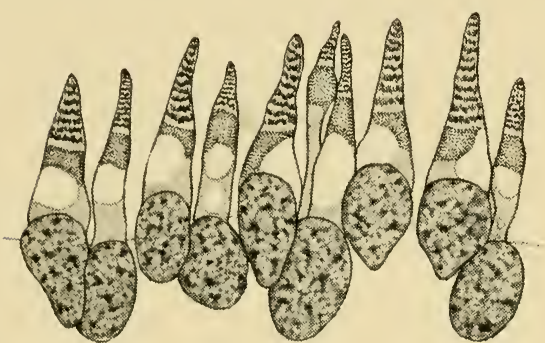

A

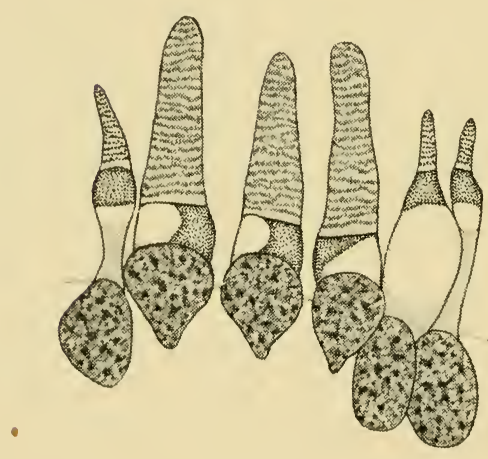

C

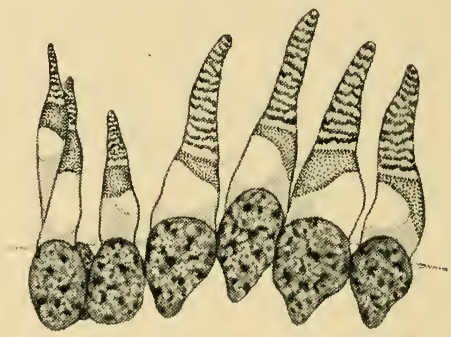

B

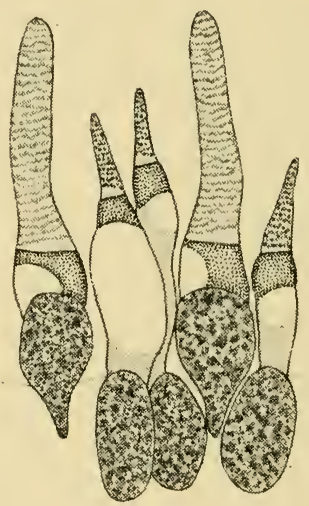

$\mathrm{D}$

FIG. 37. Later stages in the development and differentiation of the visual cells in Amblystoma punctatum. A, visual cells from the retina of a larva 21 days after tail-bud stage; B, 26 days after tail-bud stage; C, 31 days after tail-bud stage; D, 40 days after tail-bud stage. $\times 920$. (From Detwiler and Laurens, 1921, J. Comp. Neur., v. 33.)

of the inner segment, appears to be of cytoplasmic origin and is not extruded from the nucleus as asserted by Cameron (op. cit.). Distal to this globule there appears a mass of deeply staining granules in iron-hematoxylin preparations (Figure 36, A). These granules contribute mainly to the formation of the ellipsoid of the inner segment and to the granular material of the outer segment (Figure 36, C, D). 
They have been regarded by Cameron as pigment from the pigment epithelial layer ingested under the influence of the original globule. Our observations are not in support of this

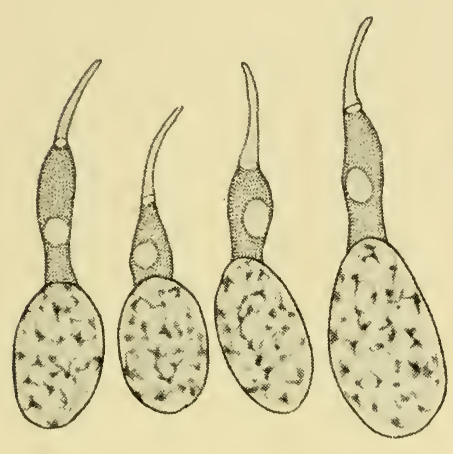

A

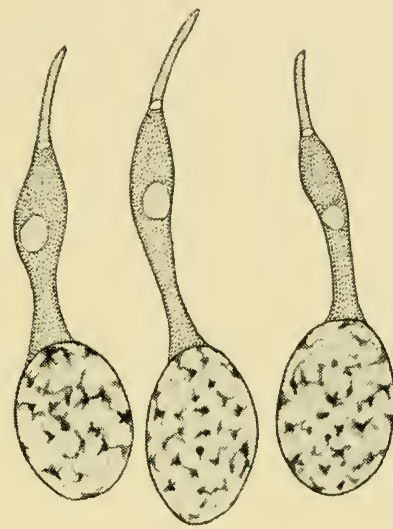

$\mathrm{B}$

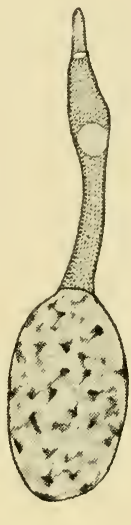

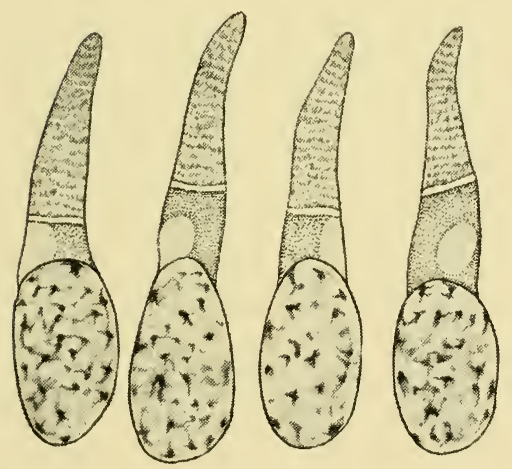

$\mathrm{C}$
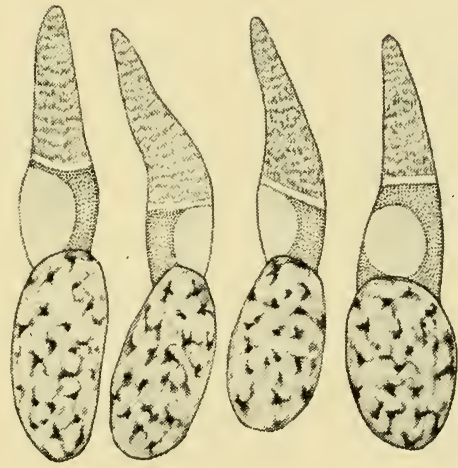

D

Fig. 38. Cones and rods from an Amblystoma larva at stage 44 (just prior to the feeding stage) showing contraction of the cone myoids in the light (A) and elongation of the cone myoids in the dark (B). The rod myoids fail to show any differences between the light-adapted condition (C) and the darkadapted condition (D). $\times 1030$. (From Detwiler, 1923b, J. Comp. Neur, v. 36.)

contention, for there are protoplasmic buds without the globule which possess the granules.

Leplat (1913), who studied the development of rods and cones in the chick, also described deeply staining granules 
in the initial protoplasmic bud and regarded them as mitochondria. This author thought that the transverse striations of the external segment and its cleavage into discs were the expression of its mode of construction from mitochondria. The granules which he has called mitochondria bear a striking resemblance to those observed in the Amblystoma retina, yet in the latter, the eyes were fixed with the aid of 5 per cent acetic acid, which is a mitochondrial solvent. Our observations suggested that the heavily stained granules are elaborated in the cytoplasm of the visual cells and that the globule comes about through the partial vacuolization of the cytoplasm.

The development of the rat retina (Detwiler, 1932) offers some interesting features. The eyelids of the rat do not open until about 13 days after birth. At birth the retina has undergone very little differentiation. There is a broad zone of nuclei, corresponding somewhat to the outer neuroblastic layer of a $15-17 \mathrm{~mm}$. human embryo. This zone is characterized, during the first two days, by the presence of many mitotic figures along the external border adjacent to the epithelial pigment layer. During the third and fourth days, mitotic figures become less in the fundus, but they are still numerous as the ora is approached. The beginning of visual cell formation is evident on the fourth day by the presence of very short protoplasmic extensions into the cavity of the primary optic vesicle. These measure 1.5 to $2.2 \mu$ in length, and are seen only at the posterior pole of the eye. Also during the fourth day one can see the first indication of some separation of the broad nuclear zone into two layers - presumably the future external and internal nuclear layers. During the fifth day, mitotic figures can still be seen in the region of the fundus, but by the sixth day they have all disappeared, and they become less numerous also in the region of the ora serrata. On the sixth day the external nuclear and internal nuclear layers are completely separated by the formation of a definite external molecular zone. Rod growth proceeds very slowly. At this 
period they average about $3 \mu$ in length at the fundus and $1.5 \mu$ at the region of the ora serrata.

The visual cells develop very slowly up to ten or eleven days after birth. At nine days, the internal and external segments are discernible, and the entire rod has attained a length of about 5-6 $\mu$. From ten to sixteen days after birth,

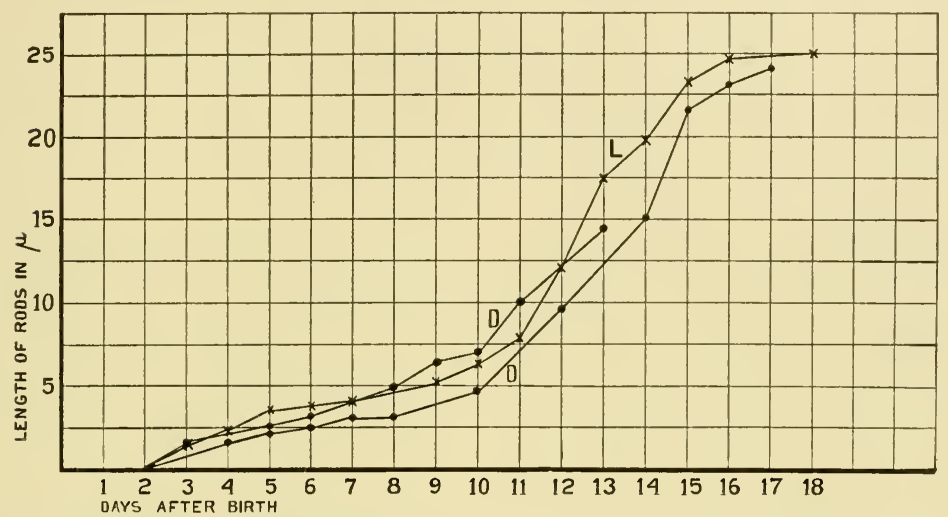

FIG. 39. Growth curves of developing rat rods in micra. D, animals reared in complete darkness; $\mathrm{L}$, animals reared in daylight.

the growth of the rods becomes greatly accelerated (Figure 39). After this period, growth is again slow. The figure shows that from 16 to 18 days after birth the rods measure from 23 to $25 \mu$. It shows also that there is no difference in the growth rate of the rods whether the young are reared from birth in light or in darkness. The rods of an adult rat were found to measure approximately $36 \mu$. Just as in the human eye, the rat eye differentiates its ganglion cells and the bipolar conducting element's prior to the visual cells.

Although in the developing rat eyes, the presence of visual purple could not be recognized in fresh, isolated retinae younger than eleven to twelve days, rats eight days old showed a definite avoiding reaction to a beam of heat-filtered light. The same observation was made by Crozier and Pincus (1927). 


\section{CHAPTER V}

\section{RETINAL STRUCTURE AND ANIMAL HABITS}

So closely correlated is the mode of life of the animal with the structure of the retina that, from a histological section, one can predict something of the habits of the animal, as well as its visual ability. The majority of vertebrates possess
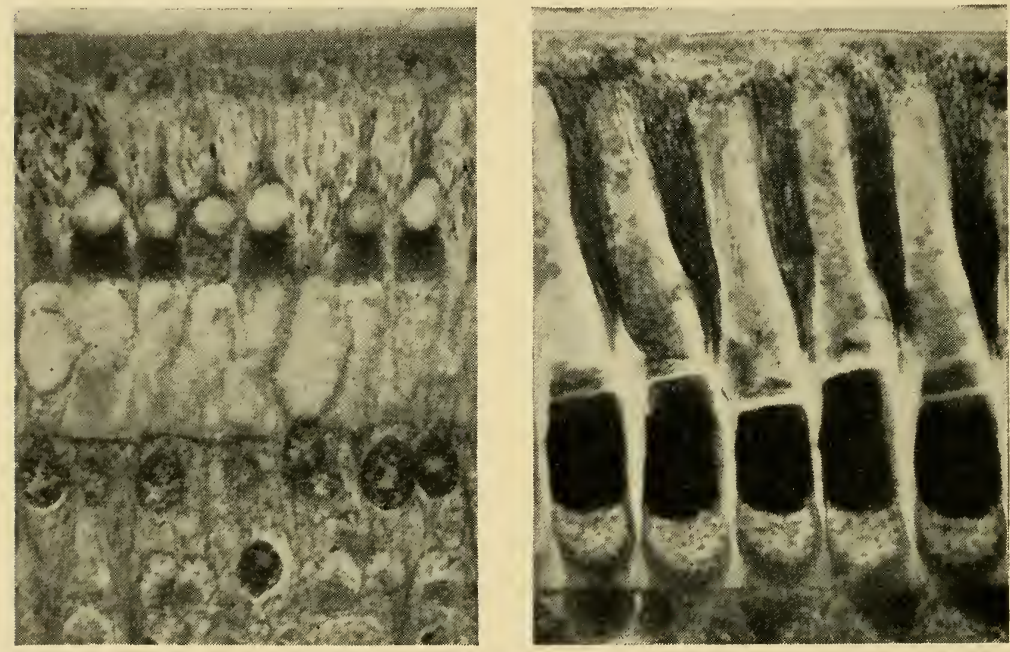

FIG. 40 (Left). Photomicrograph showing the essentially cone-bearing retina of the turtle (Chrysemys picta). Note large paraboloids and oil globules. $\times 850$.

FIG. 41 (Right). Photomicrograph showing pure rod retina of the nocturnal lizard (Gecko). × 850 .

rods and cones and are equipped for both day and night vision. Many animals, however, possess only one type of visual cell and accordingly are spoken of as diurnal (cone bearing) or nocturnal (rod bearing).

This condition is well illustrated by the reptiles, among which are many forms which have cones only, and are diurnal in their habits. In this group may be placed the diurnal 
lizards, round-pupilled snakes, and possibly some turtles. The geckos, however, which belong to the lizard family, are primarily nocturnal in their habits and possess a pure rod retina. Examples of the essentially cone-bearing retina of the turtle (Chrysemys picta), and of the pure rod retina of the gecko, are shown respectively in Figures 40 and 41.

With respect to the turtles, it has been generally held that they possess cones only. Walls (1934a) says that in the photophobic forms such as the limicolous Chelydra, many rods are present, but that very few rods are found in the sunloving genera such as Chrysemys. According to him (1942b) rods are more numerous in the actively nocturnal Pseudemys than in Chelydra. It is of interest in this connection that Crozier, et al. (1938-1939), who studied the flicker response function for Pseudemys, report this retina as possessing cones only.

In my earlier work on the turtle retina (Detwiler, 1916) I described the presence of single and double cones and the absence of rods in Chrysemys picta. A critical re-examination of the sections reveals the presence of an occasional element which lacks an oil drop, but which possesses a paraboloid and a broader outer segment than the typical cone. This element may well be a rod, but one must not confuse it with the 'accessory' member of a double cone which also lacks an oil globule, but which possesses a paraboloid and

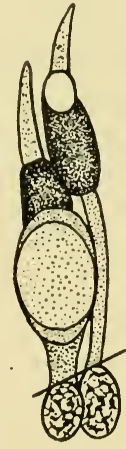

A

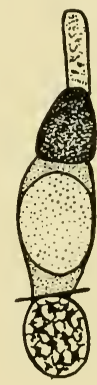

B

FIg. 42. A, double cone from the retina of the diurnal turtle (Chrysemys picta); B, rod from same retina. $\times 480$. an external segment somewhat larger than that of the principal cone (Figure 42, A). This very sparse rod-like element is shown in Figure 42, B.

Crocodiles and alligators possess a double retina which is very rich in rods. Their eyes possess also a light reflecting tapetum (Laurens and Detwiler, 1921) and are particularly well adapted for nocturnal vision. 
The situation in snakes has been studied by Walls (1932, 1942b) who examined the eyes of many forms for the presence of visual purple, which is diagnostic for the presence of rods. He found visual purple in most forms with vertical pupils (vertical slit pupils being characteristic of nocturnal forms). In four vertical-pupilled forms he found no visual purple. No visual purple was found in round-pupilled forms, most of which are considered by herpetologists as strictly diurnal.

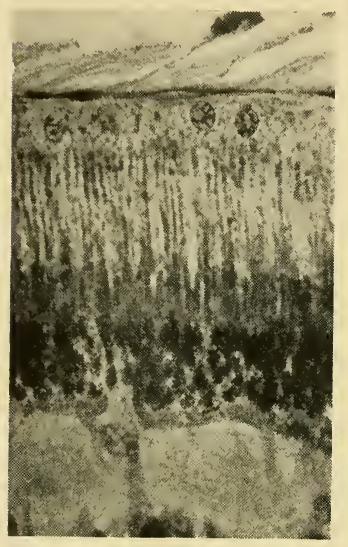

Fig. 43. Photomicrograph of the elasmobranch retina, showing absence of cones and lack of pigment in the epithelial pigment layer. $\times 455$.

Walls calls attention to the fact that some round-pupilled forms are apparently nocturnal, but these also gave negative results. He suggested that nocturnal activities in the absence of visual purple may be carried on primarily through olfaction.

Thus despite the general opinion that snakes are essentially 'cone' animals, it is seen from Walls' observations that rods occur also among many of these forms.

Most teleost fishes possess a double retina, as do amphibians, birds, and most mammals. Many fishes have a predominance of rods (particularly the pike and the eel). Among the elasmobranchs the presence of a few cones has been described (Greeff, 1899), but in my own preparations I have not been able to identify any. This characteristic rod retina shows also the complete lack of pigment in the epithelial pigment layer (Figure 43).

Among amphibians, the frog has rather small cones and large rods of two types: 1 ) violet or red rods with long cylindrical outer segments, and 2) smaller green rods with shorter external segments and long internal segments (Figure 44).

Upon the basis of structural differentiation of the retina, birds become divisible into two functional groups, viz., day birds and night birds. It is a well-known fact that ducks 
migrate during the daytime and settle down at night, whereas many birds migrate only at night. Almost yearly we hear of scores of migrating night birds which encounter death on the wing, by flying into strongly illuminated tall buildings, apparently dazzled by bright lights.

Rochon-Duvigneaud (1919), who has done considerable work on the avian retina, says that diurnal birds have a predominance of cones, and nocturnal birds have a preponderance of rods. Krause (1894), Hess (1913), and others, also found that nocturnal birds possess some cones and that the diurnal forms possess some rods.

Certainly it is within the experience of anyone who goes from the city into the country in late spring and early summer to realize that, as dawn begins, birds

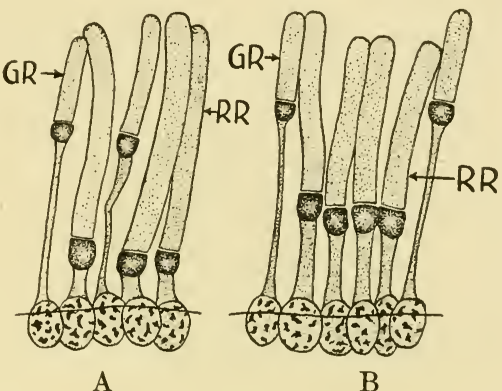

A

B

FIG. 44. Drawing showing red rods (RR) and green rods (GR) of the frog from A, dark-adapted eye, and B, light-adapted eye. $\times 850$. (From Arey, 1916, J. Comp. Neur., v. 26.) become so active as to be either a delight or a source of annoyance to the individual, depending largely upon his temperament. Who also has not experienced the pleasure of hearing the solemn call of the whippoorwill as nightfall approaches?

Because of the fineness of the visual elements and the great abundance of epithelial pigment, the finer details of the bird retina are made out with difficulty. By bleaching out the pigment, and with successful staining, one can, in most instances, obtain a satisfactory view of the visual cells. A photomicrograph of the bleached chicken retina, stained with iron-hematoxylin, is shown in Figure 45. This figure shows the presence of a goodly number of rods, which is somewhat disturbing because we all know that the fowl apparently has a poor apparatus for night vision, for he goes to roost long before man ceases to enjoy good vision. 
With regard to the owl, Katz and Révész (1914) state that the rods of nocturnal birds (owls), in bright light, are similar in function to cones. This seems to be without anatomical foundation for the reason that the retinae of such birds contain numerous cones (Garten, 1907; Hess, 1913). Whereas the owl retinae in my own collection possess numerous characteristically long rods $(80-90 \mu)$ and look like pure rod retinas, very critical examination shows the presence of small cones.
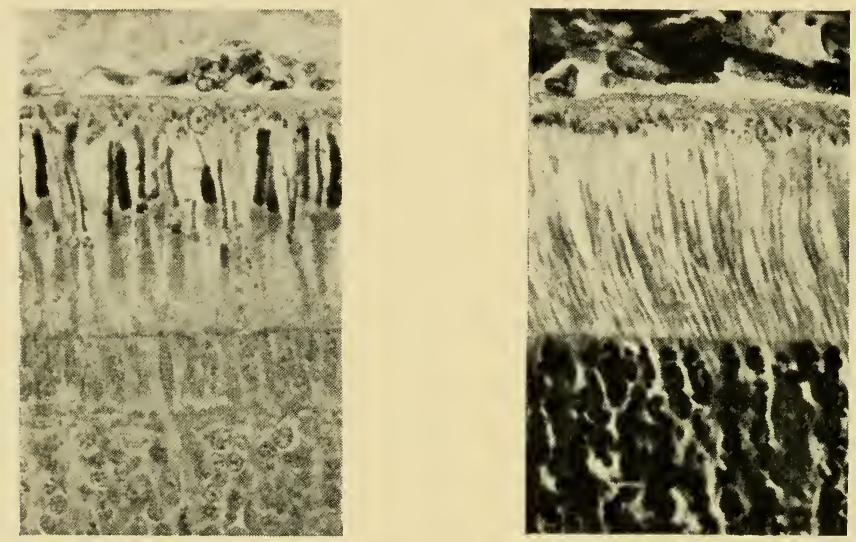

FIG. 45 (Left). Photomicrograph of the chicken retina showing rods and cones. Note large oil globules in the cones. $\times 455$.

Frg. 46 (Right). Photomicrograph of the adult rat retina. $\times 455$.

Among mammals, where the majority of forms possess both rods and cones, great variations exist with respect to the relative proportions of these two elements. Here too we find a general preponderance of rods in nocturnal forms, and an entire exclusion of cones in some cases. We are all aware from personal observations that our domestic neighbors, the rats and mice, are much more active at night than in daytime. Although it is claimed by some (Menner, 1930) that these rodents have cones, I have never been able to recognize their presence with certainty in my own numerous histological preparations of these forms. A photomicrograph of the adult rat retina is shown in Figure 46. 
The bat, a strictly nocturnal form which begins its activities with approaching dusk, possesses, according to my own findings (Detwiler, 1924), a pure rod retina. There are many other forms with which we are acquainted that are predominately nocturnal, but which can see reasonably well in daytime. The cat, for example prowls and hunts at night but not exclusively so, for we have all observed his success at hunting in daytime. The cat, however, with a vertical slit pupil, a predominance of rods, and a choroidal tapetum, is particularly well adapted for nocturnal vision. According to DeVoss and Ganson (1915), the cat is said to have very defective daylight vision and to be colorblind. Dogs appear to have a much more efficient apparatus for night than for day vision.

The retina of the horse is known to have both elements present. Horses are essentially diurnal animals, but I have found from personal experience that their capacity for nocturnal vision is probably greatly superior to that of man. The presence of a tapetum lucidum in many mammals possessing cones as well as rods shows that they are particularly well adapted for nocturnal vision.

Perhaps one of the most interesting groups of animals demonstrating the correlation between retinal structure and a nocturnal mode of life consists of the nocturnal lemurs. These include the so-called Pottos, Lorises, and Galagos. Galago mala and Nycticebus tardigradus (slow-moving loris), both of which have been under my own observation, were found to be most inactive during the daytime, and did all their feeding at night. Apparently in their natural habitat they leap from bough to bough at night and exhibit marked activity. Nycticebus tardigradus is shown in the frontispiece (Figure 1). This animal was so light sensitive that it was difficult to obtain front-view photographs. The moment one turned on a 60-watt lamp, the creature either turned its head away or buried it between its legs.

An histological examination of the retina of these forms showed the presence of narrow attenuated rods and the 
complete absence of cones (Figure 47). The rods resembled in many respects those of the rat, but were found to be slightly longer ( $v$. Table 1 ). There is no evidence of any fundus specialization such as a macula or an 'area centralis retinae' as Woollard (1925) described for the eye of the nocturnal Tarsius spectrum. The presence of an area centralis retinae in the eye of Tarsius and its supposed evolutionary relationship to the fovea, as advanced by ElliottSmith (1928) will be discussed later. Both Galago and Nycticebus showed the complete absence of epithelial pigment
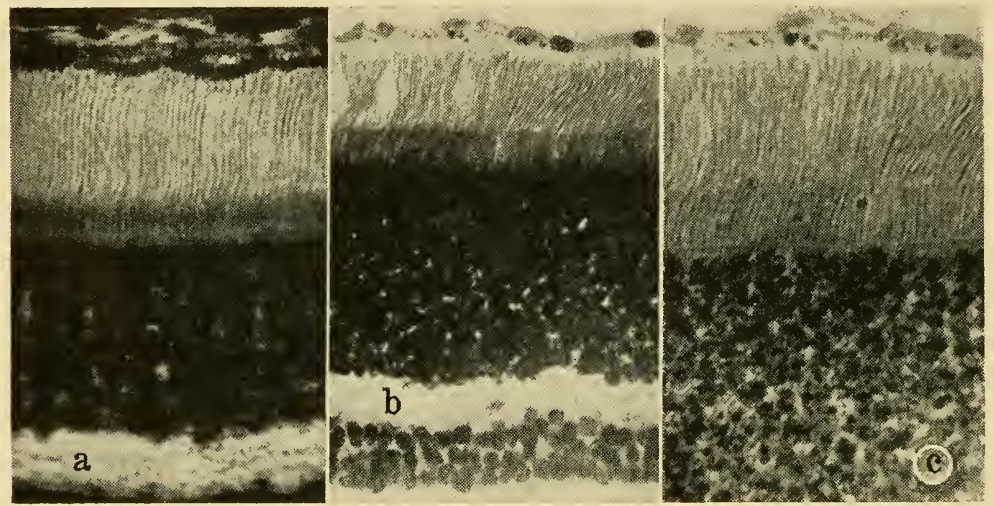

FIG. 47. a, retina of Galago mala near the ora serrata. $\times 420$. b, retina of Nycticebus tardigradus from fundus showing rods and absence of epithelial pigment. $\times 300$. c, same as b. $\times 420$. (From Detwiler, 1940, Anat. Rec., v. 76.)

in the fundus, and only as the ora serrata is approached does one see pigment (Figure 47, a). Both forms possess a fibrous tapetum lucidum (choroidal tapetum) (Figure 7).

From Woollard's description of the eye of Tarsius one is led to believe that the epithelial pigment layer contains abundant pigment for he says, "The characters of this more anterior part of the retina agree very well with the structure of the retina found in animals of nocturnal habits. This is shown in the large amount of pigment and in the outer layer of the retina." One is left in doubt as to the extent of epithelial pigment in the fundus of the retina. Extensive 
pigmentation is not specifically characteristic of nocturnal eyes, as is sometimes assumed. The great variation in the amount of epithelial pigment in the eyes of strictly diurnal and nocturnal forms, as well as in those with a dual retina, has been an obstacle to an adequate general explanation for the function of the pigment in all forms.

The amount of pigment varies greatly in animals with a pure rod retina. For example, in the elasmobranch eye it is entirely lacking; in the rat and the bat it is sparse. It is entirely absent in the greater part of the retinas of Galago and Nycticebus (lemurs); it is scant in the tapetal region of the alligator and in the region of the choroidal tapetum of mammals. Yet, in the nocturnal gecko, epithelial pigment is not only abundant but extends down between the rods so as to surround or enclose the external segments.

In addition to possessing the above nocturnal characteristics, the eyes of lemurs exhibit other noteworthy features which are in great contrast to those typifying the diurnal anthropoids and man. Chief amongst these are the very large corneas, anterior chambers, and lenses. These features are illustrated in Figures 48 and 49. The corneas are greatly curved and in meridional section occupy more than onethird of the perimeter of the bulb (particularly in Nycticebus). The lens is also strikingly large, notably in Galago where it is more than half as large as the vitreous (v. Table 2 and Figure 48). As was pointed out in the first chapter, the ciliary muscle in relation to the size of the lens is particularly large in Nycticebus (Figure 9).

The ratios given in Table 2 are not expressions of volumes, but are merely surface area ratios (as determined by the planimeter) of the various eye structures through the meridional plane. Hence they can at best be approximate only. However, the sections of the eyes shown in Figures 48, 49, 50,52 bear out in general the great size contrast between these various structures in lemurs and in the anthropoids listed. 


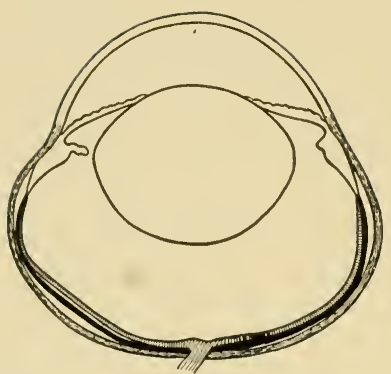

Fig. 48. Diagrammatic meridional section of the eye of the lemur (Galago mala). $\times 3$.

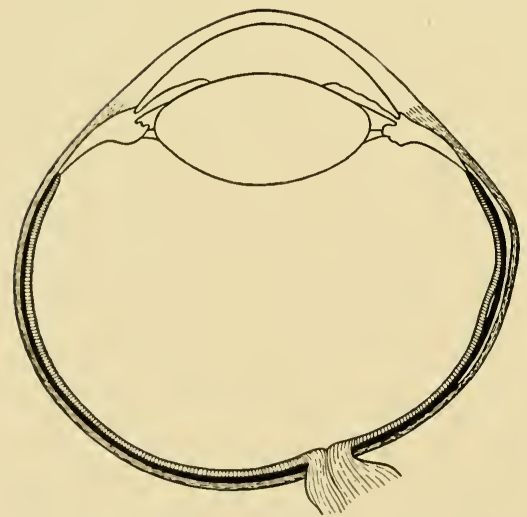

Fig. 50. Diagrammatic meridional section of the eye of the rhesus monkey (Macaca mulatta). $\times 3$.

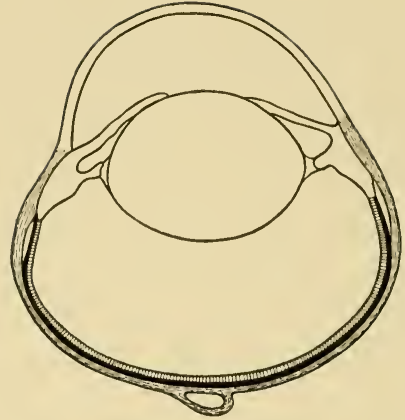

FIG. 49. Diagrammatic meridional section of the eye of the lemur (Nycticebus tardigradus). $\times 3$.

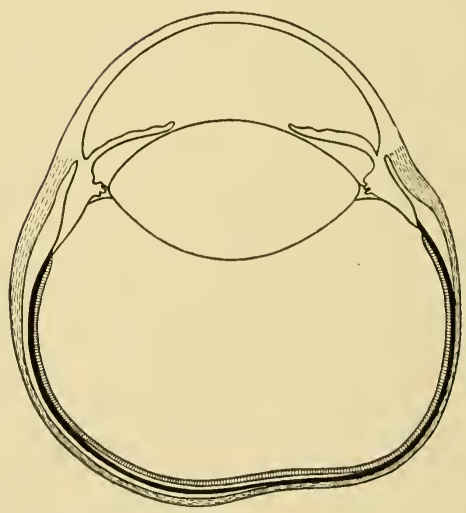

FIG. 51. Diagrammatic meridional section of the eye of the nocturnal platyrrhine monkey (Nyctipithecus). $\times 3$. 


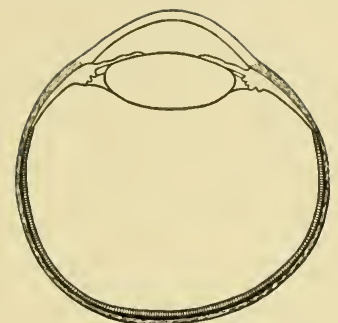

FIG. 52. Diagrammatic meridional section of the eye of the marmoset (Hapale jacchus). $\times 3$.

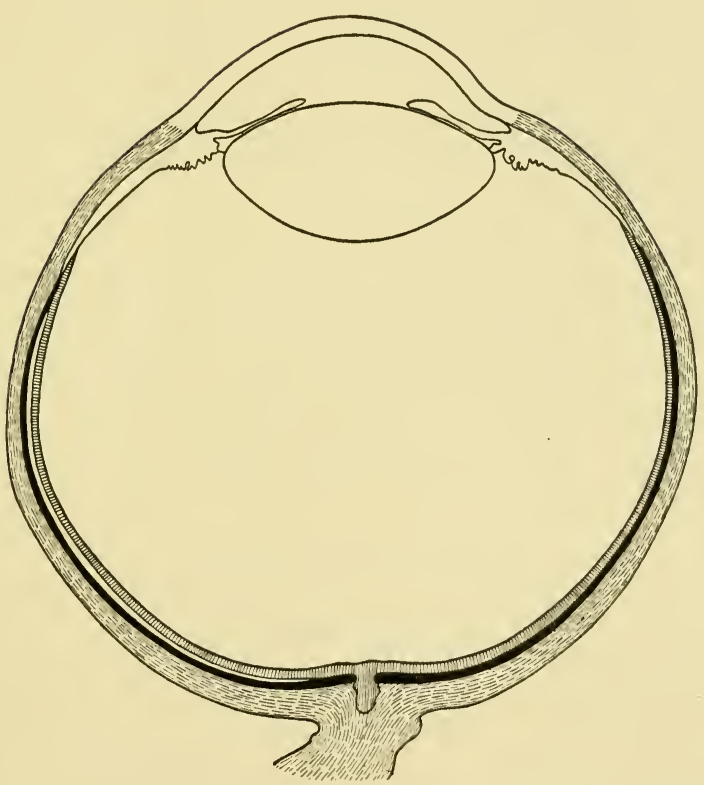

FIG. 53. Diagrammatic meridional section of the human eye. $\times 3$.

69 
TABLE 2

Showing Ratios Based upon Comparative Measurements of the Eyes of Nocturnal Lemuroids, Monkeys and Man *

\begin{tabular}{|c|c|c|c|c|c|c|}
\hline \multirow[b]{2}{*}{ Form } & \multicolumn{2}{|c|}{$\mathrm{A} \dagger$} & \multicolumn{2}{|c|}{$\mathrm{B} * *$} & \multicolumn{2}{|c|}{$\mathrm{Ctt}$} \\
\hline & $\begin{array}{c}\text { Cornea } \\
\text { to } \\
\text { Sclera }\end{array}$ & $\begin{array}{c}\text { Cornea } \\
\text { to } \\
\text { Whole } \\
\text { Eye }\end{array}$ & $\begin{array}{c}\text { Ant. } \\
\text { Chamber } \\
\text { to } \\
\text { Vitreous }\end{array}$ & $\begin{array}{c}\text { Lens } \\
\text { to } \\
\text { Vitreous }\end{array}$ & $\begin{array}{l}\text { Ciliary } \\
\text { Muscle } \\
\text { to Lens }\end{array}$ & $\begin{array}{c}\text { Ciliary } \\
\text { Muscle } \\
\text { to } \\
\text { Whole } \\
\text { Eye }\end{array}$ \\
\hline Galago & $1: 1.96$ & $1: 2.96$ & $1: 2.8$ & $1: 1.63$ & $1: 23.7$ & $1: 75.4$ \\
\hline Nycticebus & $1: 1.85$ & $1: 2.85$ & $1: 2.8$ & $1: 2.3$ & $1: 15.2$ & $1: 79.0$ \\
\hline Nyctipithecus & $1: 2.00$ & $1: 3.00$ & $1: 3.8$ & $1: 3.7$ & $1: 15.0$ & $1: 103.5$ \\
\hline Marmoset & $1: 4.10$ & $1: 5.00$ & $1: 15.2$ & $1: 9.3$ & $1: 11.0$ & $1: 128.6$ \\
\hline Rhesus Monkey & $1: 3.10$ & $1: 4.13$ & $1: 13.5$ & $1: 7.1$ & $1: 17.4$ & $1: 283.1$ \\
\hline Man & $1: 4.50$ & $1: 5.50$ & $1: 15.8$ & $1: 9.0$ & $1: 21.1$ & $1: 278.7$ \\
\hline
\end{tabular}

Galago and Nycticebus are nocturnal lemurs; Nyctipithecus is a nocturnal monkey.

* Measurements were made from sections selected from the horizontal or vertical meridian.

$\dagger$ Based on linear measurements in inches of eye sections at a magnification of $\times 11$.

** Based on planimeter surface area measurements of the various eye structures drawn at a magnification of $\times 11$.

$+\dagger$ Same as B only at a magnification of $\times 26$.

Whereas monkeys in general are essentially diurnal in their mode of life, there is one, the platyrrhine monkey, Nyctipithecus, commonly known as the owl monkey, which is strictly nocturnal in its habits. The eye of this animal was described previously by Woollard (1927) and in more detail by the author (1941).

Although Nyctipithecus is a real monkey, the general features of its eye correspond more closely to those of the nocturnal lemuroids (Galago and Nycticebus) than to those which typify the anthropoid eye. This is not because it is a primitive form, but because the eye is specialized for nocturnal vision. As is seen in Figure 51 and in Table 2, the cornea presents a marked curvature like that of Nycticebus and occupies about one-third the perimeter of the bulb. This is in contrast with the diurnal marmoset where the cornea constitutes only one-fifth of the perimeter of the globe - a condition similar to that in the human eye. The lens is also relatively large as is the anterior chamber - a 
relation which much more approaches the conditions in Nycticebus than in the diurnal monkeys (marmoset and rhesus).

The retina of Nyctipithecus is composed of fine filamentous rods which are very similar in shape, size and number to those of the nocturnal lemuroids (cf. Figures 47 a, b, and 54). There is no evidence of cones, or of any macular specialization such as a fovea or an area centralis retinae. Despite this similarity with the retina of the lemurs, there is one notable difference, viz., in the pigment epithelium. This layer in Nyctipithecus is pigmented throughout, whereas in Galago and Nycticebus it is entirely devoid of pigment in the fundus. Furthermore, there is no evidence in this form of a choroidal tapetum such as occurs in the lemur eyes.

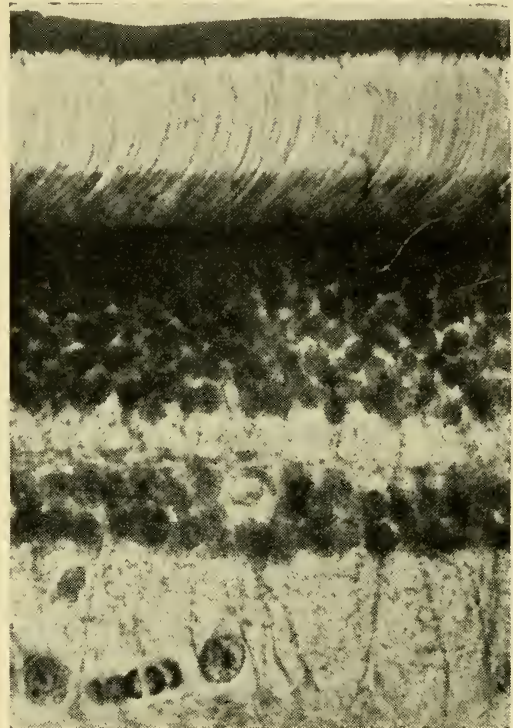

FIG. 54. Photomicrograph of the retina of the owl monkey (Nyctipithecus). $\times 485$.

Woollard (op. cit.), who described the retina of this form as a pure rod retina, without a fovea, uses his observations to support the contention that Nyctipithecus is the most primitive of the Platyrrhines. It may be pointed out that the marmoset is regarded by some authorities as a more primitive form than Nyctipithecus, yet its retina is rich in cones and it possesses a typical anthropoid fovea. It is apparent that neither the presence nor absence of a fovea and cones can be used to determine the evolutionary position of an animal. Especially is this true of the fovea, as will be discussed later.

The presence or absence of rods or cones, as well as their 
relative distribution in different forms, become matters of primary importance in a comparative study of retinal adaptation to light and darkness, and also give us a structural foundation which is prerequisite to an adequate study of the light-perceiving value of the retina as well as to the study of color vision.

From his studies upon the retina of reptiles, Walls (1934a) has concluded that many groups have become nocturnal secondarily - the condition having been brought about by a "...transmutation of some or all of the cones of an ancestral, diurnal, pure-cone retina into functional rods . . ." This 'transmutation' theory of Walls, derived from an assemblage of facts, has led him to the following conclusions:

"1) Cones have been secondarily, phylogenetically, transformed into rods during the evolution of a nocturnal habit by forms whose ancestors were strictly diurnal and pure-cone.

“2) Such transmutations have occurred many times, independently of each other, so that unrelated forms have convergently attained similar final conditions.

"3) Intermediate stages can be seen in extant genera, some of which may be in a dynamic condition with regard to a change in their habits with respect to light.

"4) The reverse transmutation - of rods into cones has probably taken place at least once, and in this case is a tertiary change.

"5) Within limits, habits rather than taxonomic position determine the visual-cell pattern which a given form will display.

“6) It was practically inevitable that visual-cell transmutation should occur in reptiles, and it is improbable that many cases will be found outside the reptilian group."

Walls stresses the point that if the rods of some animals have been derived from the cones of others, the differentiation of the two types of visual cells is more physiological than morphological, and suggests that perhaps they should be given names that have solely physiological connotation such as photocyte for the cone and scotocyte for the rod. 
Despite this point of view, this author still continues to differentiate between rods and cones upon a morphological basis.

In relation to the duplicity theory Walls says, "The occurrence of transmutation does not weaken the Duplicity Theory as a statement of physiological facts, excepting the Theory's allocation of hue perception exclusively to cones." He says that the duplicity theory will have to be put to test with respect to the implication that a cone which becomes a rod loses hue-discriminatory capacity which it may have had previously. Walls' (op. cit.) final statement is as follows: "No organon in all physiology is on a firmer foundation than is the Duplicity Theory, in its true character as a description of the functional antithesis of two physiological types of cells; but as a guide to the interpretation of habits in terms of 'structure,' the Duplicity Theory, faced with transmutation, has come to the end of its usefulness." 


\section{CHAPTER VI}

\section{PHOTOMECHANICAL RESPONSES IN THE RETINA}

One of the most fascinating yet most perplexing problems in retinal biology pertains to certain structural changes which the retina exhibits in response to illumination of the eye. The chief changes which occur are: 1) movements of the pigment in the epithelial pigment cells, 2) changes in the form and length of the visual cells, and 3) changes in the form and staining reactions of the retinal nuclear layers.

Until 1915 to 1916 the extensive investigations on this phase of retinal physiology were confined almost exclusively to European workers, and an excellent compilation of the work was published in 1907 by Garten. In 1915 Arey published an historical survey of the results in this field - the first of its kind in the English language, and he himself subsequently contributed extensively to our knowledge of these changes, particularly in fishes and amphibia (1916a, 1916b, 1919). The author investigated the problems in the eyes of reptiles (Detwiler, 1916, 1920, 1923a, 1923c) and in certain nocturnal mammals (1924).

Pigment. Variations in the degree to which retinal pigment extends down between the visual cells was noted as early as 1856 by $H$. Müller. Morano (1872) also noted the inconstancy of epithelial pigment distribution. It seems, however, that the first observations regarding the effect of light upon the retinal pigment were recorded by Czerny (1867), who found that after light stimulation the pigment epithelium and the retina were less easily separated. But to Boll (1877) and Kühne (1877), working independently, is given the credit for the discovery of pigment migration. They found that in the light, the retinal pigment of the frog extended nearly 
to the external limiting membrane, whereas in the dark, it contracted back into the body of the epithelial cells so as to form a compact band close to the choroid. Examples of the two conditions are shown in Figures 55 and 56 . Kühne predicted that a phototropic action on retinal pigment would be found in all vertebrates. His prediction has not been fully
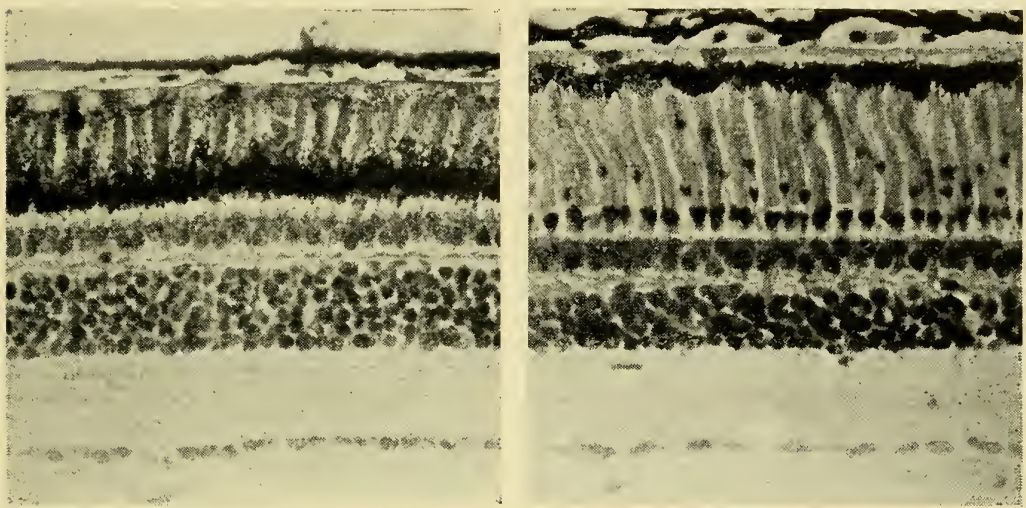

Fig. 55 (Left). Photomicrograph of light-adapted frog retina, showing expanded (migrated) epithelial pigment. $\times 267$.

FIG. 56 (Right). Photomicrograph of dark-adapted frog retina showing contracted epithelial pigment. $\times 267$.

substantiated, for these changes in the eyes of mammals and man have never been convincingly demonstrated. In the reptiles the results have been contradictory.

Among teleost fishes and amphibians a definite response of the pigment to light is general (van Genderen Stort, 1886; Pergens, 1896; Exner and Januschke, 1906; Garten, 1907; Arey, $1916 a, 1916 b)$. This has been demonstrated in amphibians by Boll (1877) and Kühne (1877), Arcoleo (1890), Angelucci (1878), van Genderen Stort (1886), Arey (1919), Detwiler and Lewis (1926), and others.

Angelucci (1878) was not able to say with definiteness from the results of a few experiments on the turtle, Testudo graeca and on the rodless lizards (L. agilis, L. muralis, and L. viridis) whether pigment migration took place or not. Boll (1881), who regarded the migration of pigment as concerned with the 
using up and regeneration of visual purple in the rods, concluded that pigment migration should not take place in a rodless retina, such as in the lizards. He did not say, however, whether it does or not. Angelucci (1894) described migration in the eye of the tortoise, Testudo marina. Chiarini (1906) demonstrated migration in Lacerta agilis. Garten (1907) was unable to obtain preparations of the retina of Emys, Chameleon, or of Lacerta, which showed consistent differences in the position of the pigment depending on whether the animal had been kept in darkness or in bright light. Hess (1910) also was no more successful than Garten in his observations on Emys europaea.

The author (Detwiler, 1916) reinvestigated these matters in the eyes of a number of reptiles (mostly upon the tortoise, Chrysemys picta, and the lizard, Sceloporus undulatus). It was concluded that in both forms, there was slight but definite pigment migration in the light $(3.6 \mu$ in the tortoise and $3 \mu$ in the lizard). It was found also that stimulation of the enucleated bulb with an induced current of moderate strength caused a migration. A still more marked migration was brought about by passing a constant current (15 to 20 M.A.) through the enucleated eye, either centrifugally or centripetally for a period of fifteen minutes.

Pigment migration was demonstrated also in the eye of the Chinese lizard, Eremias argus (Detwiler, 1923a), in the gecko, Gecko Swinhonis Güenther (Detwiler, 1923c), and in Alligator mississippiensis (Laurens and Detwiler, 1921).

In birds possessing both rods and cones, migration is usually much more extensive than in reptiles (Chiarini, 1906). Van Genderen Stort (1887) showed this to be true in both areas of the pigeon retina, where rods are present, and to a less extent in the region where they are absent. Krause (1894) also described migration in the diurnal hen, as did Angelucci (1878) for the owl.

As regards the mammals, evidence for pigment migration is very insecure. Angelucci (op. cit.) claimed to have demonstrated migration in the rabbit. Chiarini (1906) described 
slight forward migration in the retinas of dogs. Garten (1907), however, who carried out careful experiments on the ape, ox, rabbit, and rat, failed to find any evidence of migration in these forms. In the bat and rat, the author failed to find any expression of migration (Detwiler, 1924).

It can be said that the most extensive migration takes place in fishes and anuran amphibians. Also in birds, both diurnal and nocturnal, definite movements occur. In reptiles, slight migration has been demonstrated in many forms. In mammals and man it is very questionable whether pigment migration has been demonstrated definitely in any instance. Despite this lack of evidence for mammals and man, statements can be seen (principally in textbooks) which ascribe to the human eye the photomechanical responses which so typically take place in the eyes of lower vertebrates.

Following the discovery of pigment migration by Boll and Kühne, a host of workers investigated the phenomenon in the eyes of all vertebrate classes and the literature became extensive.

Cones. Not only was it found that light causes a migration of pigment, but that it also brings about positional changes in the rods and cones. The discovery that cones alter their lengths with respect to light and darkness was made by van Genderen Stort, although the earliest announcement was made by Englemann (1885) in whose laboratory the work was done. It was found that light causes a shortening, and darkness a lengthening of the cone. To the contractile portion of the inner member of the cone, Englemann applied the apt term 'myoid.' Subsequent investigations have shown that if the response occurs at all, it is the same in all vertebrates, viz., a shortening when illuminated.

The most marked positional changes of cones are found in fishes, among which the eel, however, is an exception (Garten, 1907). According to Arey (1928) the myoid may lengthen tenfold. Changes in length of the cone myoids in Ameiurus are shown in Figures 57 and 58. The extensive movement of the rods is shown also. 
Among amphibians, cone movements were clearly demonstrated in the frog by van Genderen Stort, and in the toad by Arcoleo (1890). Responses of the salamander cones were described by Angelucci (1894). His observations, however, were not supported by Garten (1907). In the eye of
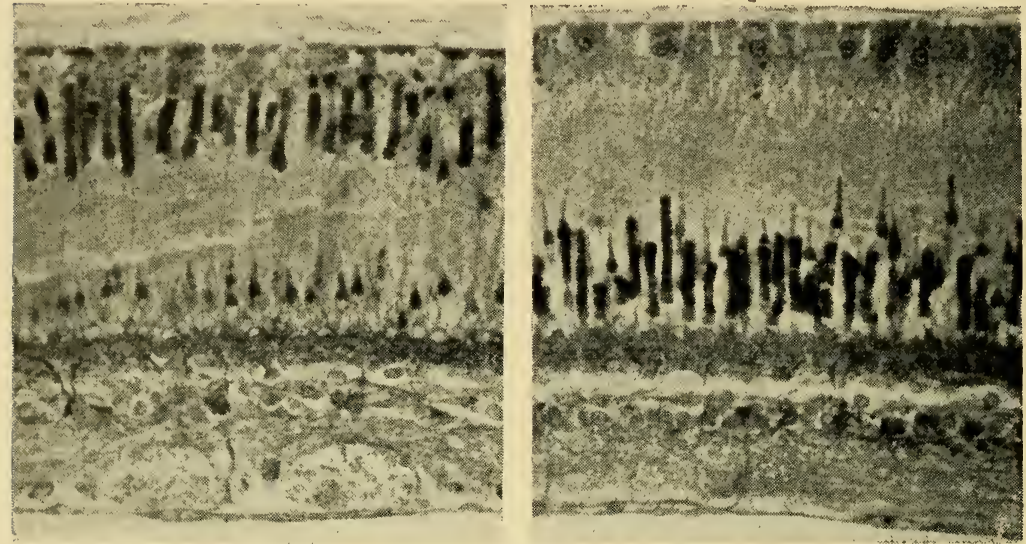

Fig. 57 (Left). Photomicrograph of depigmented light-adapted retina of Ameiurus, showing contracted cones and elongated rods. $\times 305$.

Fig. 58 (Right). Photomicrograph of depigmented dark-adapted retina of Ameiurus, showing elongated cones and contracted rods. $\times 305$.

larval Amblystoma, definite cone responses have been noted (Detwiler, 1923b).

In reptiles possessing cones only, the results have been inconsistent. Slight changes have been reported by Chiarini (1906), and by Garten (1907) for the rodless retinas of lizards and the chameleon. The author also observed detectable cone contraction in turtles and lizards (Detwiler, 1916, $1923 a, 1923 c)$.

In birds, it has been possible to compare the movements of the cones in regions with many rods (yellow field), few rods (red field), and none at all (fovea). As measured from van Genderen Stort's (1887) plates, Arey (1928) expresses the relative changes in length as follows: - fovea: red field: yellow field $=23: 32: 40$. Garten (op. cit.) substantiated this relationship and also extended his observations to the 
owl and to the hen. The owl apparently possesses very delicate cones, and the hen, although essentially a diurnal form, possesses a goodly number of rods (Figure 45).

In mammals, cone movements seem very doubtful. Van Genderen Stort claimed positional changes in the cones of the pig. Chiarini (1906) obtained negative results on the dog, and Garten working with apes suggested possibly slight changes in the region of the fovea.

Cone contraction under the influence of light is greatest in fishes; it occurs to a less extent in amphibia and in some birds. Changes in the reptilian cones are slight, but demonstrable. In mammals the evidence is most doubtful.

Rods. Angelucci (1884) was the first to observe positional changes in the vertebrate rod. He claimed that the frog myoid shortened after exposure to light. He applied the term myoid to the contractile portion of the rod as Englemann had done for the cone. Arcoleo (1890) also reported a shortening of the rod myoid in light, but Lederer (1908) challenged the results of previous investigators and claimed that the rod myoid elongates in the light, thus supporting the contention of van Genderen Stort. Arey (1916c) reinvestigated the matter in the frog, where he found that both the red rods and the green rods shorten in darkness, and concluded, "Therefore, contrary to the conclusion of the older workers, the photomechanical response of the frog's rod myoid is found to be similar to that occurring in the retinas of all other investigated vertebrates."

The most extensive changes in the length of the rod myoid occur in fishes where, as Arey has shown, the mobility may result in a 90 per cent change in length (Figures 57 and 58). His observations on the movement of the so-called green rods and red rods of the frog are shown in Figure 44.

In urodeles, the rod myoid, unlike that of the cone, fails to exhibit any positional changes to light. No movements were observed in the gecko rods (Detwiler, 1923c), but responses were found in the rods of the alligator where both rods and cones occur (Laurens and Detwiler, op. cit.). 
Earlier workers apparently paid little attention to the mammalian rod, and the data are sparse. In my own investigations I have been unable to find any responses of the rod to light in the eyes of mice, rats, and the bat (Detwiler, 1924).

It can be concluded, then, that when photomechanical responses do occur in response to light stimulation, the pigment migrates forward, the cones contract and the rods elongate or fail to respond (e.g. urodeles and mammals). In dim light or darkness the typical response is the reverse, viz., the pigment contracts, the cones elongate, and the rods contract (Figures 59 and 60).

These changes have been shown to be affected by conditions other than light and darkness. For example, temperature has been found to play a significant rôle. Arey (1916a) showed in fishes that both in light and darkness, the retinal pigment exhibits greater expansion at low than at high temperatures. He showed also that in darkness, the retinal pigment of the frog undergoes striking expansion between $0^{\circ}$ and $14^{\circ} \mathrm{C}$ and between $19^{\circ}$ and $33^{\circ} \mathrm{C}$. At the intermediate temperatures of $14^{\circ}$ to $19^{\circ} \mathrm{C}$ it is highly contracted. Temperature was found to have no effect upon light-adapted eyes. Essentially similar results were obtained on the frog by Detwiler and Lewis (1926). In these experiments, however, we found that in previously light-adapted eyes, the retinal pigment will contract after one hour's exposure to darkness between the temperatures of $18^{\circ}$ and $23^{\circ} \mathrm{C}$, thus showing a wider temperature range for contraction than found by Herzog (1905) and Arey (op. cit.).

Arey found that the cone myoids of fishes in the dark shorten at low temperatures and elongate at high temperatures. Temperature was found to be ineffectual in the light. He showed also that the rods of fishes elongate at high temperatures and shorten at low temperatures, but the extent of change is much less than in the cones.

Carbon dioxide and ether were found to block completely the movements of all retinal elements in the intact and 
excised eyes of fishes, whether in light or in darkness. Chloretone and urethane on the other hand had no effect.

Arey (1916b) also investigated the rôle of the integrity of the central nervous system upon the photomechanical responses with interesting results. Observations are recorded in the literature which ascribe a central control over retinal pigment and visual cell movements - particularly in the frog. It has been claimed also that stimulating agents applied to one eye will induce changes in the other; and some workers even claimed that this interrelation was independent of the brain, and persisted after optic nerve section distal to the chiasm. This effect upon the non-stimulated eye was supposed to be brought about by what Englemann (1885) called retino-motor fibers. He made the statement that illuminating the skin of dark-adapted frogs would bring about changes in the pigment and the cones. This observation, however, was not borne out by the experiments of Fujita (1911) and Arey (1916a). Hamburger (1889) and Fick (1891) showed that after section of the optic chiasm of the frog, light and dark adaption proceeded as in animals with an intact optic nerve. This suggested that photomechanical changes were responses to direct light stimulation and in no way dependent upon the integrity of the optic nerve. Nahmacher (1893) showed, however, that stimulation of the optic chiasm of the frog by salt crystals caused changes in the cones only if the optic nerve was intact.

In discussing the various observations, Arey says, "Hence it seems probable that, in the frog, the retinal elements are capable of more or less independent movement, but over this is superimposed a nervous (efferent) control, the nature of which is not altogether evident."

Working upon three teleost fishes (Abramis, Fundulus, and Ameiurus), Arey (1916b) showed that in Ameiurus pigment migration and visual cell movements failed after section of the optic nerve, whereas in Abramis and Fundulus the retinal photomechanical changes were not effected by similar surgical interruption. Since in Ameiurus the typical photo- 
tropic changes occur in the excised eye, it became apparent that the failure, after optic nerve section, is due to some mechanism either in the eye muscles or in the blood vessels which normally exert an inhibitory action on the retinal movements. This was found to be the case, for when all the eye muscles and blood vessels of a dark-adapted catfish were cut, allowing only the optic nerve to be intact, typical responses to light ensued. It was shown further that after section of the superior oblique and the external rectus muscles (those innervated by the trochlea and abducens nerves) no pigment migration occurred. However, when the remaining eye muscles were severed also (those innervated by the oculo-motor nerve) pigment migration ensued as in a normal animal. It became apparent then that the inhibitory mechanism exists in association with these muscles (or their blood vessels) which are supplied by the III nerve. It was shown further that under these conditions, electrical stimulation of the peripheral stump of the optic nerve can overcome this inhibition. Hence experimental evidence was produced offering physiological proof for the existence of functional efferent fibers in the optic nerve. By the balanced interaction of these fibers with a second extrinsic set of nerve fibers (possibly ciliary nerves), which exert an inhibitory influence upon the retinal elements, the normal phototropic movements of the retinal elements are brought about. In summing up Arey says, "It is probable that efferent impulses in the optic nerve fibers do not directly stimulate the retinal elements to motion, but rather such impulses have an indirect action, possibly by counteracting, that is, blocking, the tonic inhibition exerted by the second system of nerve fibers. If these efferent optic nerve fibers fit at all into the scheme of 'nerve components', they may be designated as visceral efferent elements."

This inhibitory mechanism was not met with in Abramis and Fundulus. Apparently, it is lacking also in the frog eye. It is possible, therefore, that it may be peculiar to Ameiurus alone. 
Some relatively recent observations of importance upon the phototropic responses of the catfish eye were made by Welch and Osborn (1937). They found that the extreme dark-adapted retina is obtained only if the eyes are prepared during the night. If the animals are allowed to remain in the dark, the visual cells and epithelial pigment may approach the typical light conditions at the time of sunrise and tend to return to the dark position at sunset. They found, further, that with constant illumination, from 24 to 48 hours, the rods and cones maintained throughout the typical light condition. When kept in darkness, however, for a similar period, the positions of the rods and cones are characteristic of extreme dark adaptation during the night, but during the day they approach the conditions characteristic of light adaptation. If the existence of a similar persisting diurnal rhythm should be demonstrated in the eyes of other forms, it becomes apparent that many measurements which have been made in the past on positional changes in the pigment and visual cells may become subject to considerable modification.

Arey and Mundt (1941) have shown that when Ameiurus is kept constantly in the dark, the cones show greater elongation (extreme dark adaptation) at midnight than at noon. This rhythmic alternation in total darkness imitates the stronger diurnal rhythm in nature, but is "independent of actual alternating daytime light and nighttime dark." They found that this rhythm will continue at least for four days. They did not produce convincing evidence of a comparable diurnal rhythm for rods and retinal pigment under conditions of constant darkness.

The existence of diurnal rhythms in the activities of animals and plants is a field of biological investigation which is relatively new and should yield many interesting and fundamental facts in the physiology of the organism.

In addition to the effects upon the epithelial pigment and the positional changes in the visual cells, light has been shown also to bring about changes in form, position, and 
staining reactions of the ganglion cells and of the nuclei of the inner and outer nuclear layers.

The effects upon the nuclei of the cones as observed by various workers are summarized by Garten (1907). Chiarini (1906) found that in the lizard the cone nuclei became longer and narrower. Birch-Hirschfeld (1906) found that light caused a difference in the volume of the cone nuclei of the pigeon; he also claimed that light caused the nuclei to approach nearer to the external limiting membrane.

Light was found to decrease the power of the nuclei in the outer nuclear layer to stain. This was shown by Pergens $(1896,1897,1899)$ in Leuciscus rutilis, Mann (1895) in the dog, Birch-Hirschfeld (1900) in the dog and cat, and (1906) in the pigeon, Sgrosso (1905) in the frog, and by Garten (1907) in Ceropithecus, Macacus rhesus, fishes (Abramis and Leuciscus), Salamandra, frog, and the owl. Chiarini (1904 and 1906) claimed doubtful differences in Leuciscus and absolutely no difference in the frog, lizard, crow, and dog.

As regards the nuclei of the inner nuclear layer, Mann (1895) found that in the dog, illumination of the eye for 12 hours caused a decrease in the staining reaction of the inner nuclear layer, and a decrease or dispersion of the Nissl substance in the cytoplasm of the ganglion cells. Bach (1895) could find no differences in the staining reactions of the inner nuclear layer or of the ganglion cells in light- or dark-adapted eyes of the rabbit. Birch-Hirschfeld (1900), however, did find differences between the light and dark eyes of rabbits and of dogs. The nuclei of the inner layer in the dark eyes are rounder; in the light, more oval. In the ganglion cells of light-adapted eyes the Nissl bodies have indistinct boundaries, with the protoplasmic background very diffuse. In the dark eye, the Nissl bodies possess sharp distinct outlines. In Leuciscus, Chiarini (1904) found no decrease of chromatin in the inner nuclear layer after illumination, and hardly noticeable changes in the ganglion cells. He also failed to observe any effects upon the inner nuclear layer of reptiles, birds, and mammals. 
Schüpbach (1906) found no differences in the inner nuclear layer or in the ganglion cells between light and dark eyes of pigeons. Birch-Hirschfeld (1906), however, was able to demonstrate clearly that the ganglion cells showed a distinct decrease in the number of Nissl bodies and an indistinctness of their boundaries in the light eye. Carlson (1904) also showed the same for Phalacrocorax penicillatus, and finally Sgrosso (1905) found in the frog distinct differences in the staining reactions of the inner nuclear layer between light and dark eyes.

The author investigated these matters in the eyes of the turtle (Chrysemys), and whereas no effect of light could be found on the form and volume of the ganglion cells, it did reduce or disperse the amount of chromatin and Nissl substance, so that the cells stained less darkly and more diffusely (Detwiler, 1916; Figures 8 and 9). 


\section{CHAPTER VII}

\section{ON THE SIGNIFICANCE OF PHOTOMECHANICAL MOVEMENTS}

Much has been written concerning the functional significance or adaptiveness of the photomechanical changes in the vertebrate retina. With respect to the various theories Arey (1915) made an apt statement when he said ". . . that many such explanations reveal the resourcefulness of the human mind rather than the ingenuity of nature." But as he pointed out further, suggestive and stimulating hypotheses serve their purpose by thus bringing the possible dynamic interrelations to light.

The various explanations held to account for these photomechanical changes have never fully satisfied all the conditions, mainly due to the lack of uniformity of the light response throughout the vertebrate groups.

Kühne (1878), who is named as one of the discoverers of pigment migration, thought that the chemical products resulting from the decomposition of retinal pigment through the action of light, stimulated the visual cells sufficiently to initiate the sensation of vision. In cases of albinism where pigment is absent, other light stimulating substances were postulated as present, in addition to visual purple and the brown pigment, fuscin. Essentially similar views were postulated by Boll (1881) and by Gad (1894).

Ayres and Kühne (1878) argued for a relationship between retinal pigment and the regeneration of visual purple. They compared the pigment cell to a gland and supposed that the epithelial pigment cells secreted visual purple. The main support for their theory was based upon their observation that the use of pilocarpin was found to reduce considerably the time required for regeneration of visual purple in the 
dog and the rabbit. A similar view was held by Kolmer (1909), who described the presence of 'droplets' on the outer segments of the rods - supposing them to be concerned with the visual purple functions and having their origin in the pigment epithelial cells. His observations will be discussed more fully later. Comparable results were obtained on the frog by Dreser (1886).

Kühne (1879) stressed the parallelism between the time needed for the regeneration of visual purple in the frog and the length of time for dark adaptation, as indicating a significant relationship between the two. Chiarini (1906) expressed the view that the function of the migrated pigment is nutritive to compensate the rods and cones for losses which they sustain during functional activity. Herzog (1905) considered the migration of pigment as useful in storing up radiant energy so that the "dark heat waves" thus made available would accelerate the chemical processes taking place in the visual cells.

Herzog (1905) and Exner and Januschke (1906) maintained that these retinal changes represent a mechanism for adapting the eye to daylight and to twilight vision. In dim light or in darkness when the rods are capable of being stimulated, the pigment moves back (contracts towards the perikaryon of the epithelial cell) and leaves free the spaces between the rods, resulting thereby in a less complete insulation of these cells. Under these conditions, with the entrance of a small amount of light, the part played by the individual rods in the perception of light, owing to refraction and diffusion, is greater than if they were covered by a thick mantle of pigment, in which case only the light which passes through the retina in the direction of the long axis of the rods could enter them. The presence of a light reflecting tapetum further enhances favorable conditions. The cones, under these conditions, are not functional, by reason of their high thresholds. Consequently they elongate and move out of the way. The rods contract so that optimum conditions are presented for their stimulation. 
In bright light the pigment moves distally, surrounding and protecting the rods (which have a low threshold, and which have been rendered sensitive by the accumulation of visual purple in the dark) from too strong light stimulation. This is accomplished by the pigment absorbing the direct and scattered light. The rods elongate, while the less sensitive

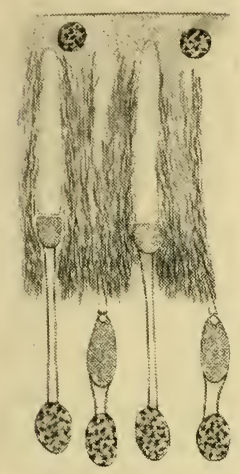

FIG. 59 (Left). Theoretical light-adapted retina, showing migrated epithelial pigment, elongated rods, and contracted cones.

FIG. 60 (Right). Theoretical dark-adapted retina, showing contracted pigment, elongated cones and contracted rods.

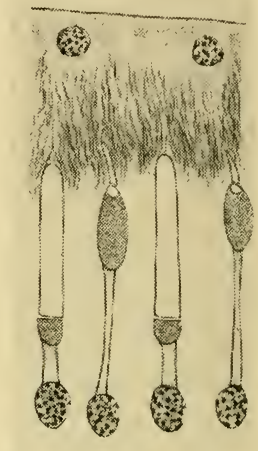

cones are drawn out of the pigment by their myoids and are then made freely accessible to the stronger light stimulus - thus presenting optimum conditions for their stimulation. The theoretical situation is illustrated in Figures 59 and 60 and an actual condition as observed by Arey in the eye of Ameiurus is illustrated in Figure 61, A and B.

It must be assumed in such a theory that the cone cells remain elongated in very dim light, but, according to Garten, working on Abramis, this is not true; consequently the theory doesn't hold. Garten who weakened the theory of Exner and Januschke (1906), maintained that the function of the expanded pigment is to absorb all light which might escape from the visual cells by refraction; that were it not for the optical isolation of the visual cells by the pigment, a great deal of light would be scattered in all directions, on account of the large ellipsoids (fishes) and the strongly refractive oil drops (amphibians, reptiles, and birds) and would stimulate the neighboring rods and cones. In the long slender rods such as occur in mammals, however, 
total internal reflection prevents this dispersion of light. This is supported by the fact that in most rod retinas where the rods are long and slender, the pigment is either very sparse or entirely wanting. Certainly in the mammalian eyes which I have examined (mouse, bat, guinea-pig, dog, rabbit, cat, monkey, man) the rods are slender elements with
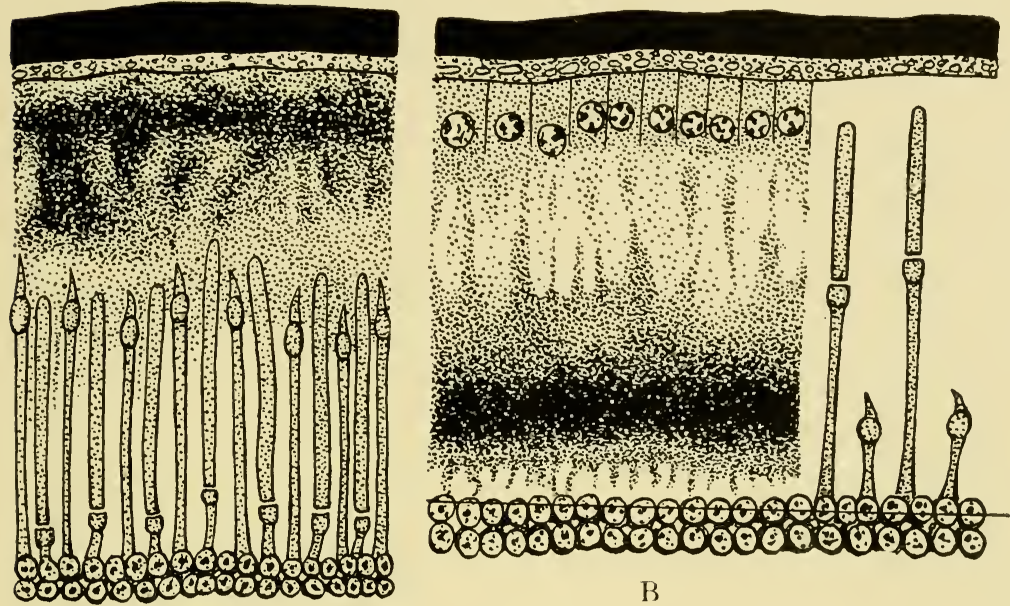

$\mathrm{B}$

A

Fig. 61. A, dark-adapted, and B, light-adapted retina of Ameiurus nebulosus. In $\mathrm{A}$ the pigment is withdrawn toward the choroid, the cone myoid elongates, the rod myoid shortens. In B the pigment moves forward toward the external limiting membrane, the rods elongate and the cones shorten. (Redrawn from Arey, 1919. J. Comp. Neur., v. 30.)

no refractive bodies, and the pigment is not only scarce but is entirely wanting in the regions of those eyes which possess a tapetum. In nocturnal lemurs (Galago and Nycticebus) which have long attenuated rods and possess also a choroidal tapetum, there is complete absence of pigment in the fundus. On the other hand, the owl monkey, Nyctipithecus, which has a pure rod retina similar to the nocturnal lemurs, does have a narrow band of pigment extending throughout the epithelial pigment layer.

When we examine the gecko where the rods are large, with prominent 'barrel-shaped' ellipsoids and a large paraboloid, 
there occurs a heavy zone of pigment which almost completely enshrouds the outer segments (Figure 41). This seems to be in marked contrast with all other nocturnal forms. One can hardly see the reason for the heavily pigmented retina of this form unless it be to absorb refracted light. In the gecko, as in other reptilian forms with only one type of visual cell, slight migration of the pigment occurs. Such meager migration would seem to have no adaptive value as was postulated by Exner and Januschke for eyes with both rods and cones (e.g. alligators). Garten's theory necessitates the assumption that there is no need for pigment migration in an eye containing exclusively cones or rods. Despite this, however, moderate migration has been demonstrated in the retinas of reptilian forms possessing cones only. If the pigment is of value in absorbing refracted light and thus enhances independent stimulation of the individual cells, it would seem to be more important in eyes with cones - particularly those with large ellipsoids, paraboloids, and oil drops, which are supposed to be highly refractive. Lateral losses from the cones of mammals, therefore, should be les's serious because they lack oil drops and paraboloids. Fishes which have abundant migratory pigment also lack paraboloids, but here the ellipsoid by reason of its shape may be more highly refractive. It is interesting to note in this respect that pigment is very much heavier in fishes than in mammals. The evidence for a correlation between the amount of pigment and the refractivity of the visual cells is not conclusive, but seems worthy of investigation. It would seem not so important to isolate the individual rods, for rods apparently need all the available light as evidenced by the presence of a reflecting tapetum and the absence of pigment in many nocturnal forms. Cones, for the sake of more efficient visual acuity, may require more thorough optical isolation by the pigment.

Despite all the theoretical considerations, however, there exist so many disparities, that an all-inclusive theory does not seem to cover all the conditions. 
Walls (1928) suggested that correlated with the backward orthogenesis in the pigment migration is a forward progress in the development of the iris reaction. He calls attention to the fact that in fishes " . . the pupil is practically stationary; in the amphibians it expands and contracts within narrow limits; in reptiles the response is about the same as in amphibians, while in mammals and man the response is very rapid and very extensive." Walls thus assumes that the pupillary reaction is a much more efficient mechanism for protecting the retina against too much light than are the sluggish pigment and cell movements. As valuable as this suggestion would seem, it may be pointed out that in birds, where the iris response is more rapid than it is in the mammals, the pigment is not only abundant, but undergoes extensive migration. Furthermore in the gecko which is entirely nocturnal, and which possesses a vertical slit pupil, rapidity of the iris reaction surpasses that of the human and approaches that of birds (Rochon-Duvigneaud, 1917), yet here too we find very heavy pigment processes surrounding the outer segments of the rods (Figure 41). Walls (op. cit.) recognizes this condition in the birds, and says: "It must be admitted that the situation in nocturnal animals where both kinds of phenomena are lacking, and in birds, where both kinds of phenomena are present to a marked degree, is disturbing to this suggestion, but the nocturnal animals have little or no need for either mechanism, and the eyes of birds are so aberrant in many respects that perhaps we are safe in dismissing them in this instance." It hardly seems justifiable to the author to regard the birds' eyes as being so aberrant. They possess a double retina, many have a fovea, some have a double fovea, and they are known for their high visual acuity. With respect to their structural characteristics they are more typical than are the eyes of many other animals where, for example, only one type of visual cell is present. Furthermore, facts do not bear out the statement that nocturnal animals have little or no need for a pupillary mechanism. 
Most nocturnal animals have either a vertical or horizontal slit pupil which probably allows for more extensive closing than a round pupil - hence a greater protection against bright light. The gecko which is a typical nocturnal animal has a vertical slit and a rapidity of closure surpassing that of man. It is also said that the spotted night snake (Hypsiglena), a nocturnal form, exhibits an extremely rapid pupillary response.

Despite all of the theories, none of which seem to satisfy all the conditions, it would seem most logical to regard the pigment processes as useful to some extent in optically isolating the visual elements. In 1916a, Arey summed up his observations by saying, "Although the movements of the visual cells and retinal pigment, when present, may have a certain unknown significance in connection with the mechanism of light perception, such movements can be interpreted at present only in terms of protoplasmic responses to definite stimulating agents." In this sense the pigment cells respond to light as do skin melanophores, viz., an expansion and streaming of the pigment. The adaptive significance of these phototropic responses in the retina still remains an intriguing problem, with no all-inclusive solution. 


\section{('HAPTER VIII}

\section{THE RELATION OF RETINAL STRUCTURE TO VISUAL ACUITY}

Although there are a number of factors involved in the matter of visual acuity, one of these, and an important one, pertains to the size and numbers of the visual elements. We are all aware from an elementary acquaintance with photography that the fineness of detail which a photosensitive surface can register depends upon the fineness of the emulsion. If the receiving elements are fine and close together, one obtains much greater detail than when the particles are large and far apart. This condition is exactly what one finds in the retina. A low visual acuity signifies that the average distance between the active retinal elements is large, whereas a high visual acuity means that the distance is small. Since this is true, it is obvious that vertebrate retinas must vary greatly in this respect. Since also the number of rods and cones is fixed structurally in any given region of the eye, it is apparent that the number of elements per unit area must vary functionally in order to mediate the great variation in visual acuity accompanying changes in illumination. This has been shown by a number of investigators.

Working with brightness discrimination Koenig (1894) found that the whole range of intensities visible to the eye is made in 572 discrete steps in intensity recognition. About 30 of these are mediated by the rods and the remainder by the cones. Hecht (1928) has critically analyzed Koenig's findings, adding physiological support to these data.

With very low illumination, vision is a function of the rods. As more and more rods reach their thresholds and become functional, visual acuity increases. With further 
increase in illumination the first cones begin to function, but visual acuity is still mediated by the rods because there are more active rods than cones. Presently, with still further illumination, visual acuity becomes determined entirely by the cones and the gradual augmentation of the number of functional cones will continue until all are active and no further change in visual acuity is possible. The distribution of rod and cone physiological thresholds, assumed by Hecht (1928) to describe quantitatively the data of Koenig, is given in Figure 29. Here it is seen that a few of the cones have thresholds lower than the rods. We see, therefore, that the requirements for the large variations of visual acuity cannot be met entirely by the structural make-up of the retina alone and that they are realized fully by the visual cells possessing different thresholds. Since, however, the resolving power of the retina depends also upon the size and average distance apart of the photosensitive elements, it is apparent that vision must vary greatly in different forms.

Most teleost fishes have a predominance of rods, which are large, although their lengths vary with illumination. Measurements of the rods of Fundulus and Ameiurus have shown them to be $90-100 \mu$ long in light-adapted eyes and as short as 30-35 $\mu$ in dark-adapted eyes. In deep-sea fishes where one would expect rods to prevail, they apparently are fewer (Karsten, 1923) or are lacking altogether (Brauer, 1908). Many teleost cones are also large, but there are exceptions as there are with the rods. In order to compare the relative sizes of the rods and cones in the various vertebrate groups the reader is referred to plates I and II from Arey (1928) where the visual cells pictured have been drawn to a uniform magnification. Whereas, in general, the visual cells in fishes are large, those of the dim-light fishes are very small. One can say, however, that fishes in general possess visual cells, particularly the rods, which are distinctly larger and farther apart than in mammals. The same can be said of amphibians as far as rods are concerned. The frog has 
small cones and large rods of two types, viz., the red rods and the green rods (Figure 44). Anyone who has made repeated examinations of the retina is well aware that the visual cells of fishes and amphibians are much easier to study than are those of birds and most mammals, by reason of their larger size and greater distance apart.

In the reptiles one meets with a variety of conditions. In the predominantly cone retinae of diurnal turtles and in the pure cone retinae of the diurnal lizards, the cones near the ora serrata are short and stout with considerable

FIG. 62. Photomicrograph showing area centralis retinae of the turtle, Chrysemys picta. Note local thickening of the external nuclear layer caused by increased number of cones. $\times 360$.

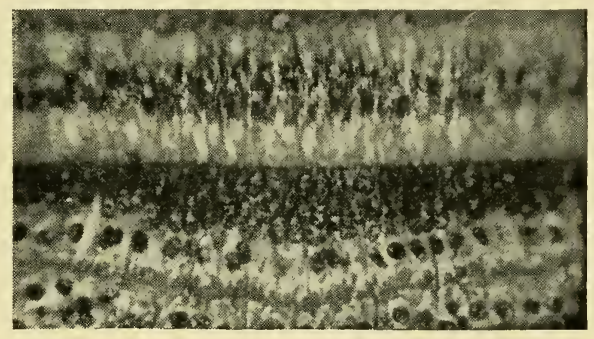

intervals between them. As one passes from the ora towards the fundus, they become more numerous per unit area, and gradually longer. In the turtles one sees a small region in the visual axis of the eye where the cones are distinctly smaller and more numerous than in the remainder of the retina. The increased numbers of nuclei, therefore, cause a bulging and thickening of the external nuclear layer. This region is termed the area centralis retinae (Figure 62) and is regarded as the forerunner of the appearance of the fovea. The concentration of numerous thinner and more closely packed elements in this region, no doubt presents a structural condition enhancing visual acuity. In the diurnal lizards the fundus specialization has gone further and here one meets a highly developed fovea centralis where the cones become long, closely packed, and filamentous. This is particularly striking in such forms as the horned toad and the chameleon (Figure 68). In the latter form the foveal cones reach a length of about $100 \mu$ which surpasses that of 


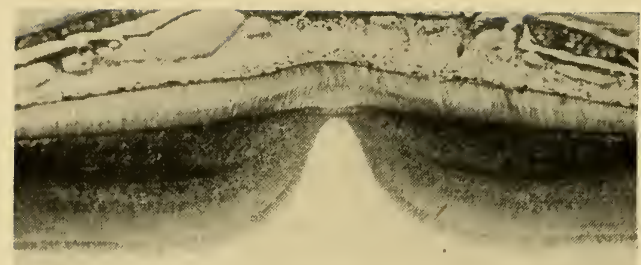

Fig. 63. Photomicrograph of the fovea centralis of the chameleon. $\times 40$.

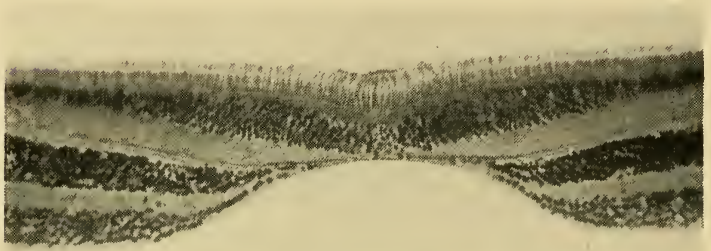

FIG. 64. Photomicrograph of the fovea centralis of the marmoset. $\times 80$.

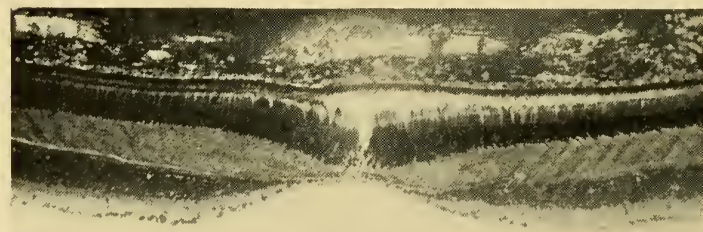

FIG. 65. Photomicrograph of the fovea centralis of the rhesus monkey (Macaca mulatta). $\times 56$.

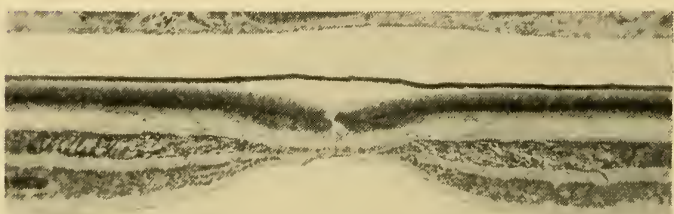

Fig. 66. Photomicrograph of the human fovea. $\times 40$. 


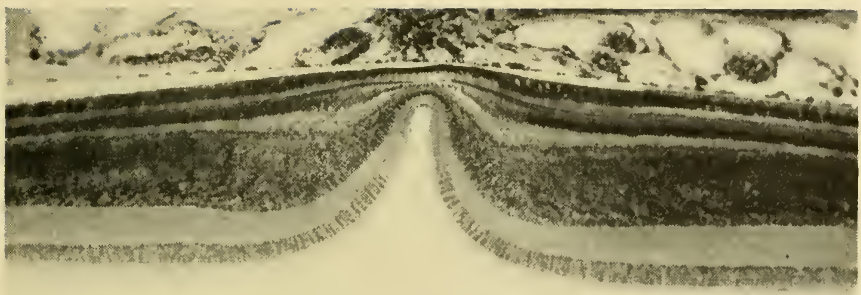

Fig. 67. Photomicrograph of the central (nasal) fovea of the hawk. $\times 50$.

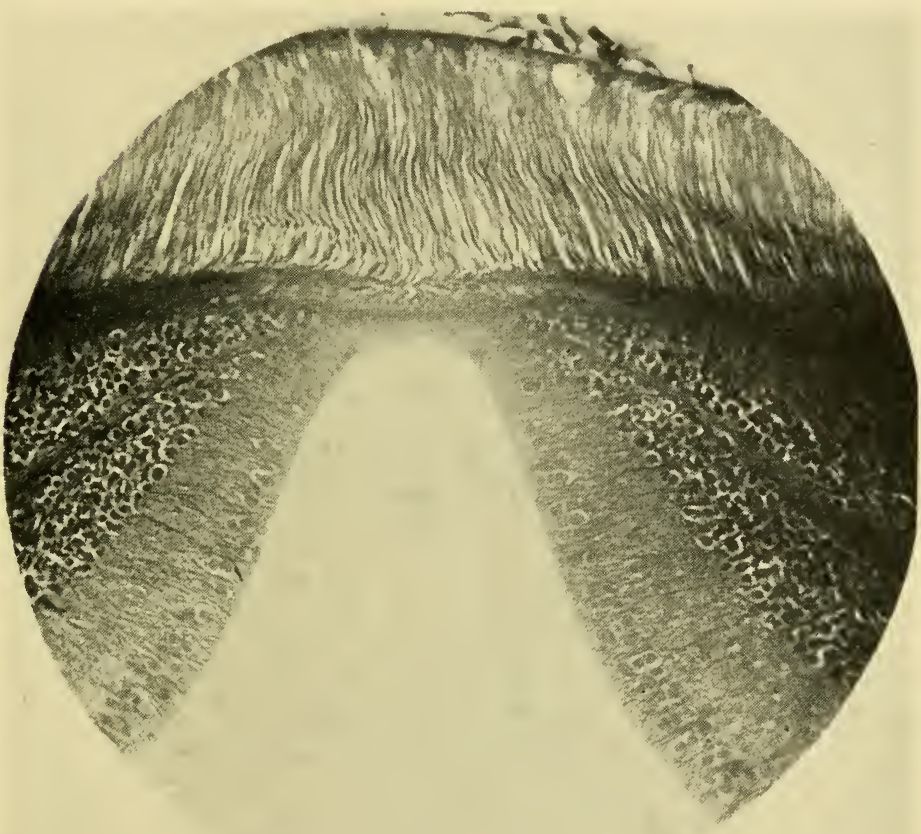

FIG. 68. Photomicrograph of the depigmented fovea centralis of the chameleon. $\times 200$.

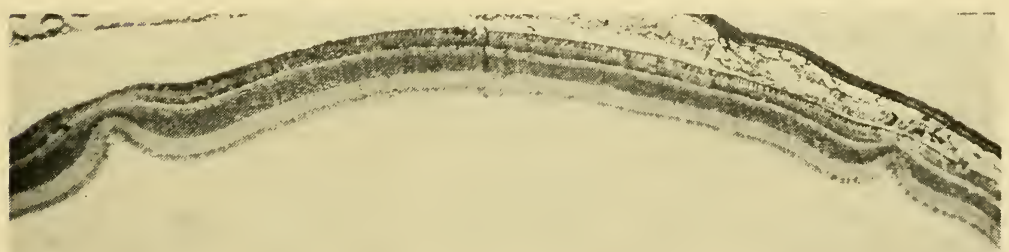

FIG. 69. Photomicrograph showing double fovea of the swallow. $\times 25$. 
the human foveal cones. So deep is the foveal depression that all of the nuclear layers are displaced laterally (Figure 68). In the anthropoid and human fovea, the depression is not nearly so deep, the external nuclear layer is continuous over the foveal pit, and the cones are not as slender nor as closely packed as in the chameleon fovea. The comparisons are seen in Figures 63-66. When one compares the highly developed fovea of the chameleon and other diurnal lacertilians with that of the human, one can only be led to believe that in the former, the conditions for high visual acuity are more nearly met than in man.

In the alligator, Chievitz (1889) first described a tapetum extending through the entire upper half of the retina in the form of a bright band. In this band he found a fovea in the form of a very superficial narrow furrow with thickened edges, and running horizontally across the entire tapetum about $1 \mathrm{~mm}$. from its lower edge. In my own studies on the alligator retina, I described the tapetum and relative distribution of rods and cones, but did not observe the striped fovea. My sections, however, were cut vertically and probably outside the foveal area.

In some birds a similar situation is encountered. Casey Wood (1917) describes a band-like area in the retina with which is associated a well-defined fovea or macula. To this form of avian retinal differentiation he gave the term infulamacular fundus - (infula, meaning band or ribbon). This type is exemplified by the Greater Yellow-legs (Totanus melanoleucus). In this class the band encloses in some part of its course a single fovea which is found on the nasal side of the fundus (Figure 70). In some forms this band-like area contains two macular regions which may be joined. This type he has called the infulabimacular fundus. An ocular specialization of this kind is illustrated by the common Old World flamingo (Figure 71). It is well known that the majority of birds possess a central (nasal) fovea. The diurnal rapacious birds and the swallow possess both a central and a temporal fovea. The latter in some instances is said to 
be more highly developed than the former. The highly developed nasal fovea centralis of the hawk, and the double fovea of the swallow are shown respectively in Figures 67, 69 .
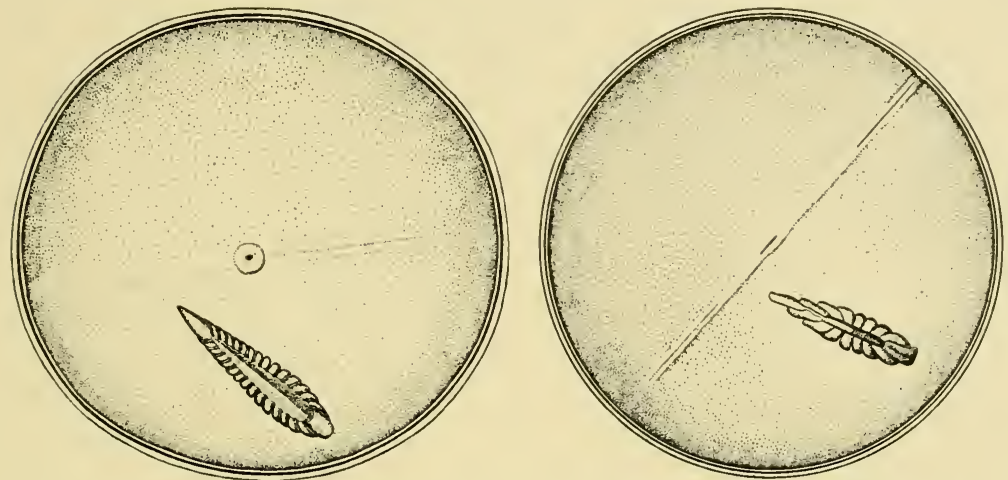

Fig. 70 (Left). Infulamacular fundus of the Greater Yellow-legs (Totanus melanoleucus) showing band or ribbon-like area with single central fovea. (From figure 114, The Fundus Oculi of Birds, Casey Wood, 1917. Lakeside Press, Chicago.)

FIG. 71 (Right). Infulabimacular fundus of the Old World flamingo (Phoenicopterus roseus) showing two macular regions. (From figure 115, Casey Wood, 1917.)
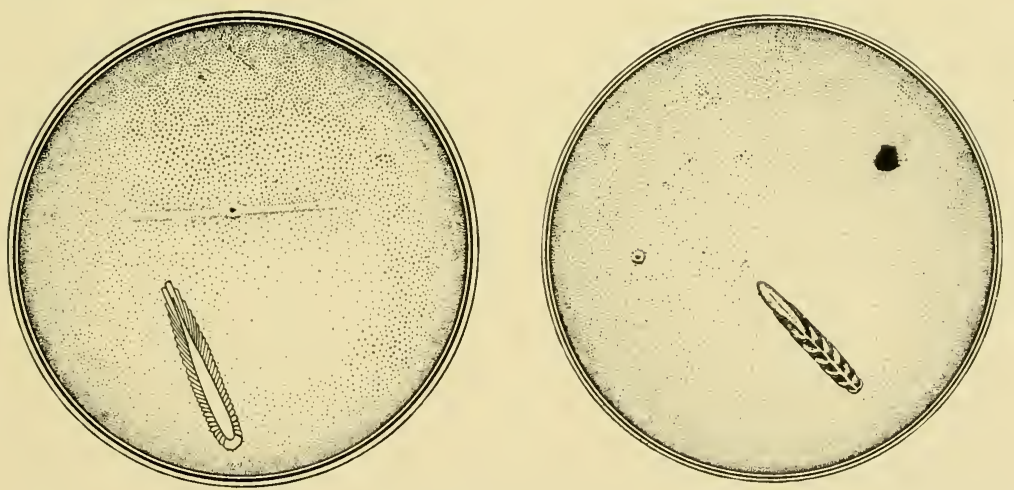

Fig. 72 (Left). Fundus oculi of the American crow (Corvus americanus) showing single central fovea. (From figure 142, Casey Wood, 1917.)

FIg. 73 (Right). Fundus oculi of the European barn owl (Strix flammea) showing single temporal fovea. (From figure 112, Casey Wood, 1917.)

Wood (op. cit.) and Slonaker (1897) pointed out that in birds with a single fovea, this structure is generally placed about the center of the retina, slightly above and towards 
the nasal aspect of the optic disc. Such a type is found in the common crow (Corvus americanus) and is illustrated in Figure 72. An exception to the rule of the nasal position
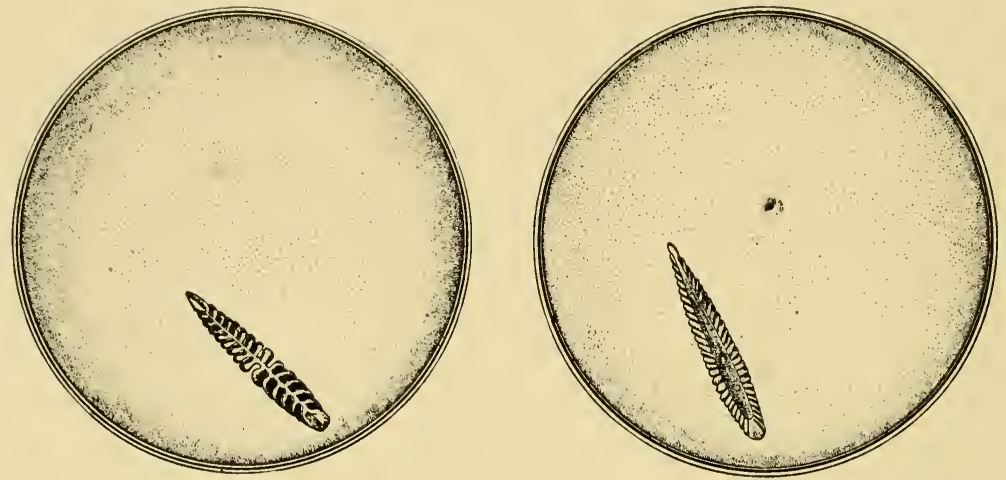

Fig. 74 (Left). Fundus oculi of the California valley quail (Lophortyx californicus vallicola) showing absence of fovea. (From figure 110, Casey Wood, 1917.)

FIg. 75 (Right). Fundus oculi of the stellar jay (Cyanocitta stelleri) showing single nasal fovea. (From figure 111, Casey Wood, 1917.)
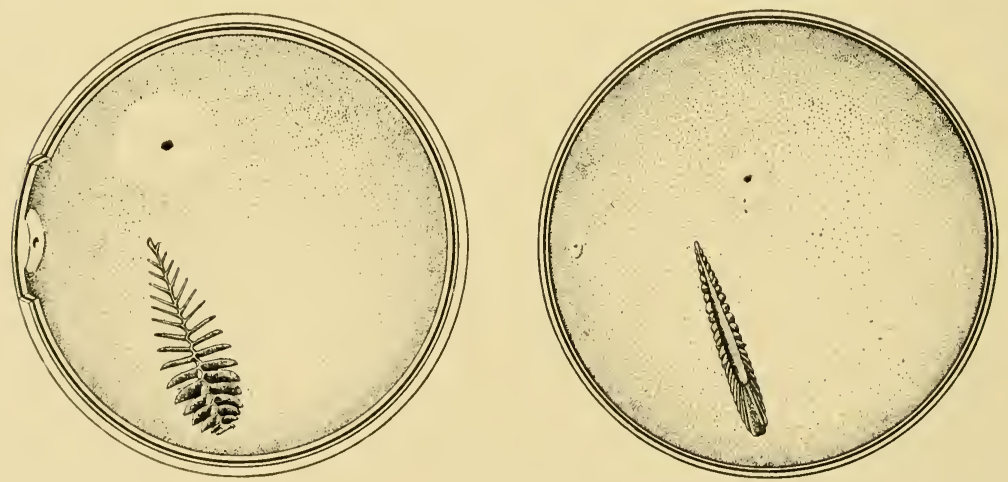

FIG. 76 (Left). Fundus oculi of the British kingfisher (Alcedo ispida) showing nasal and temporal foveae. (From figure 113, Casey Wood, 1917.)

FIg. 77 (Right). Fundus oculi of the California shrike (Lanius ludovicianus gambeli), showing nasal and temporal foveae. (From figure 141, Casey Wood, 1917.)

of the single fovea apparently exists in the owls, where a single, deep fovea enriched by a round sharply defined area is located above and on the temporal side of the optic disc 
(Figure 73). According to Wood, this position is very similar to that found in man, and in this respect the general conformation in Strigiformes resembles the human eye more closely than does any other order.

Birds of prey and swift fliers show the best marked and greatest variety of macular regions. The sparrow hawk serves as a good illustration. In this bird each macular region shows a well-marked nasal fovea and a temporal fovea which are connected by a short band-like area. According to Wood, in such forms the nasal fovea is invariably the deeper and sharper of the two and is probably used for distinct and binocular vision. Although Wood says that in the bimacular fundus, the nasal fovea is probably used for binocular vision, it is apparent from Figure 78 that the temporal fovea is used for this purpose. The nasal fovea is surrounded by a large macular area and is situated at about the center of the retina. The temporal

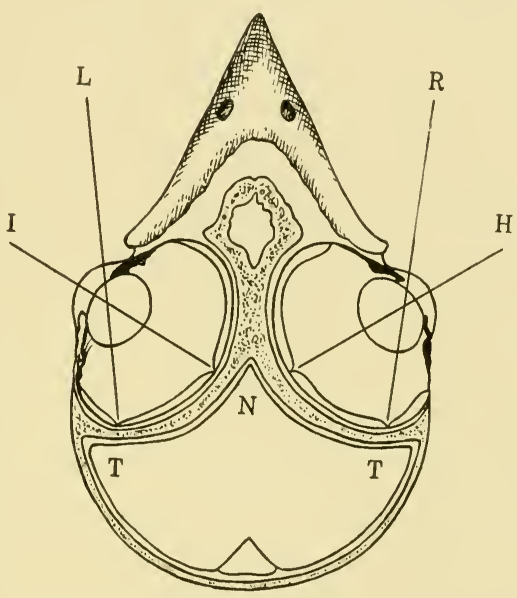

FIG. 78. Section through the head of the white-bellied swallow (Tachycineta bicolor), showing nasal and temporal foveae. NI, NH, visual axes of foveae nasales. TR, TL, visual axes of fovea temporales. (Redrawn from figure 5, Casey Wood, 1917.) fovea is shallower, surrounded by a small area, and is placed near the periphery of the retina, lower than the nasal fovea but about the same distance from the optic disc. The terns and swallows, which are swift fliers, present much the same foveal configuration as the hawks. This disposition of the 'areas' for acute vision assumes this particular form in birds that take their prey on the wing, or by darting upon it from a distance.

Wood has classified the various 'areas' for distinct vision in birds as follows: 
1. The Amacular Fundus. Those which show no evidence of a central macular region either with the ophthalmoscope or in preserved material. This is illustrated by the California quail (Figure 74). Upon histological examination such fundi exhibit a retinal area (area centralis retinae) more specialized for distinct vision than the surrounding retina. Apparently such fundi are rare and found mostly in domestic birds. Wood quotes Slonaker as having described a shallow illdefined area centralis in the turkey and guinea hen, and a very slight thickening in the cock (Gallus).

2. The Nasal Monomacular Fundus. This is situated in the center of the retina and is found in the majority of birds (Figure 75).

3. The Temporal Monomacular Fundus. This form is found exclusively in the owls and is illustrated by the European barn owl in Figure 73.

4. The Bimacular Fundus. Here two foveas occur - the nasal, which is the principal one and usually deeply marked, and a less developed temporal fovea. This is illustrated by the kingfisher in Figure 76 and also by the swallow (Figure 69).

The bimacular fundus exhibits two varieties, viz., those with a more highly developed nasal macular region where the fovea is sharp and well defined (e.g. swallow), and those where the temporal macular region is more marked, and with a deeper fovea than in the nasal region (e.g. hawks).

5. Band-like Area Centralis. In this band or stripe there exists a well-defined fovea or macula. This is the type which Wood has designated as the infulamacular fundus. It is illustrated in Figure 70. The fovea included in this band is found on the nasal side of the fundus.

6. Infulabimacular Fundus. In this type of specialization, two macular regions are either joined or are associated with the band-like area (Figure 71). This form of specialization presents two varieties. The one is characterized by a welldefined nasal macula with a deep fovea situated near the center of the retina, and a shallow temporal macula. These two are joined by a short ribbon-like area which does not 
extend beyond either macular region (e.g. sparrow hawk). In the other, the band-like area encloses a nasal macula, whereas the temporal macula lies above and apart from these (e.g. tern).

It is seen from the above, that in birds macular specialization reaches the highest degree and in no other animals do we encounter such highly developed visual acuity. In these macular regions in birds the cones are attenuated structures closely packed. According to Rochon-Duvigneaud (1919) there are 1,000,000 cones per sq. mm. at the fovea in Buteo. This would necessitate practically a solid aggregation of cones $1 \mu$ in diameter. In humans, the distance between the centers of two adjacent cones is said to be about $2.5 \mu$, which if correct, would mean about 160,000 cones per sq. mm. at the fovea (v. Chapter III, p. 40). Based upon the fineness and average distance apart of the photosensitive elements (as pointed out in the beginning of the chapter), we would expect the visual acuity of hawks to be far superior to that in man, and I think experience bears this out. Just how much greater the resolving power is in a unit area $1 \mu$ in diameter as compared with $2.5 \mu$, I am not in a position to say. Apparently the diffraction pattern limits the resolution and this depends upon such factors as size of aperture and distance from the lens to the receiving surface. This is a matter for the physicist to evaluate. However, it is apparent from the discussion and the illustrations that the fovea of reptiles and birds is a more highly developed structure than in anthropoids and man.

The eyes of diurnal reptiles and birds possess a peculiar structure known as the pecten, which projects from the optic disc into the vitreous. In the reptiles it exists typically in the form of a cone (Detwiler, 1920). In most birds it is present as a highly vascular comb-like structure containing abundant pigment (Figures 70-77). This structure has been assumed by many to play a nutritive rôle for the vitreous. It is present in most elaborate form in birds which are noted for their high visual acuity. What rôle, if any, this structure 
plays in the visual function of these forms is a matter which apparently has not been determined with finality. ${ }^{1}$

The majority of mammals apparently show some degree of fundus specialization, although in some there is no evidence of a central area (e.g. rat, mouse, sheep). When present this 'area centralis' may be band-shaped as in the horse, but usually it is round. In the horse the band-shaped 'area' is said to be temporal and eccentric. In the fox and hare it occupies an inferior position. Apparently among mammals no true macula with a fovea is encountered until we reach the monkeys. Most monkeys, the great apes, and man possess a fovea of the concaviclivate type (Figures 64 to 66 ) in contrast to the convexiclivate type of the sauropsida (Figure 63).

We see from the above that in most vertebrate animals some degree of retinal specialization occurs in the fundus for increased visual acuity. This may be very slight in some forms and involve merely a greater crowding of the elements per unit area in this region. In others, the crowding is sufficiently extensive to cause a bulge or local thickening of the retina - particularly in the external nuclear layer. When this situation is obtained we speak of it as the area centralis retinae. This may be essentially circular (e.g. turtle, Figure 62) or in the form of a stripe or ribbon-shaped area (some birds). Further specialization for increased visual acuity constitutes the foveas which are found in some saltwater fishes, diurnal lizards, birds, monkeys, the great apes, and man.

Visual acuity or detailed vision must be affected also from a structural standpoint by the relative numbers and connections of the bipolar cells, association cells and ganglion cells in the retina. The numerical relation of these neurones to the photoreceptors varies greatly in different forms. It has been generally considered that individual cones make connections with single bipolar cells, whereas many rods

${ }^{1}$ For a discussion of the various theories regarding the significance of the pecten, v. Walls, G. L., $1942 a$, "The Vertebrate Eye," pp. 648-659. 
collect into a single bipolar cell (Figure 24). The former condition is supposed to present a structural situation providing for isolated conduction of the cones - thus favoring greater visual acuity at high intensities. The latter is said to enhance summated conduction of the rods. This concept, however, has become subject to modifications as a result of recent researches of Polyak (v. Chapter II, p. 30). In connection with the structure of the retina it is of interest to compare the width of the external nuclear and internal nuclear layers. These have been measured in twenty-seven different forms (see also measurements by Menner, 1929) and are given in Table 1. Photomicrographs showing some of the differences are seen in Figure 16. From an examination of the table and figure, it becomes obvious that in birds, where visual acuity is known to be great, the internal nuclear layer is extremely wide as compared with the width of the external nuclear layer. This is especially striking in the hawk, the sparrow and the goldfinch, where the internal nuclear layer is from five to six times as wide as the external. This great difference must mean the presence of many association cells, be they amacrine or horizontal cells. In the human retina (paramacular region) the internal nuclear layer is narrower than the external. Diurnal reptiles, which are likewise known for their creditable visual acuity, show the increased thickness of the internal nuclear layer, but the difference, in general, is not as great as in birds.

It is interesting to note also that in the dimlight fish (Ameiurus) where behavior is dominated largely by taste buds, the internal nuclear layer is very poorly developed. It measures $7.8 \mu$ in width as compared with $122 \mu$ in the sparrow.

Among the mammals, conditions vary considerably. In those which are primarily nocturnal, the internal nuclear layer is considerably narrower than the external. Whereas the contrast in thickness of the two layers is considerable in both the hamster and the cat, the bat shows almost a $1: 1$ relation. This form, however, which is nocturnal, has 
rods which are not nearly as numerous per unit area as in the cat. The contrast is marked in the lemurs and is greatest in the owl monkey (a strictly nocturnal form) where the external nuclear layer is between three and four times as thick as the internal (Figure 16). If the greater thickness of the internal layer over the external means anything in terms of enhanced visual acuity, and one can only be inclined to believe that it does (as judged from the extremes of conditions in diurnal birds as compared with nocturnal lemurs), then the marmoset and the rhesus monkey may have a slightly more efficient visual apparatus from the standpoint of conduction than the human retina. In connection with the suggested possibilities for increased visual acuity on such a basis, it would be interesting to compare the visual acuity of the owl and gecko with other forms. In the owl, which is regarded primarily as a nocturnal form, the width of the internal nuclear layer is relatively almost as thick as in the diurnal birds. The owl also has long closely packed rods which reach more than $80 \mu$ in length. The gecko, which is nocturnal in its habits, has a broad internal nuclear layer as compared with the external, and the conditions approach that found in the diurnal turtle and the horned toad. The gecko has large rods which are relatively widely spaced as compared with those in nocturnal mammals which would indicate a lower visual acuity from the standpoint of the fineness and distance apart of the receiving elements. Might this be compensated for, in any way, by a more efficient conducting and association mechanism? If the individual rods in the gecko should be connected with more bipolar and association cells than they are in nocturnal mammals, then the visual apparatus of this form might be expected to be more efficient - just as it seems more efficient in birds on this basis.

There is no absolute proof at hand to argue for a greater or less efficient visual mechanism on the basis of the width of the internal nuclear layer. It would seem, however, on a priori grounds that this must be considered as a factor. 
It cannot be sheer accident that diurnal birds with their high visual acuity should have such a strikingly broad internal nuclear layer, and that in the nocturnal mammals it should be so narrow as compared with the external nuclear layer. Conduction must be influenced also by the number of ganglion cells in relation to the other two nuclear layers. In the nocturnal mammals with very broad external nuclear layers, much narrower internal nuclear layers, and still thinner ganglion cell layers, impulses from a great many photoreceptors must be concentrated into a few final paths. It reminds one of a series of reducing valves. In the diurnal lacertilians and birds, relatively fewer receptors must make connections with many bipolar and association cells in this internal nuclear layer, which in turn also must discharge into a reduced number of ganglion cells. In all retinas, whether diurnal or nocturnal, whether the photoreceptors are very numerous per unit area or less so, the number of ganglion cells is never as great as the visual cell nuclei or the nuclei of the internal nuclear layer. Visual acuity, therefore, from a structural standpoint, appears to be influenced not only by the fineness and distance apart of the receiving elements, but it is apparent that the great differences existing in the numbers of the conducting and association elements may also play an important rôle. 


\section{CHAPTER IX}

\section{ON THE SUPPOSED EVOLUTIONARY RELATION- SHIPS AND THE FUNCTIONAL SIGNIFICANCE OF THE FOVEA}

In an address by the late G. Elliott-Smith (1928) on the subject "The New Vision," he attributes the changes which occur in the structure of the parts of the anthropoid brain concerned with vision, as due to the origin of the macula which is also, according to him, responsible for the profound evolution of the nature of vision in apes and man. In this connection he says, "The crucial importance of the spectral tarsier for the study of these problems is now widely recognized. This interesting creature has almost, though not quite, developed into a monkey. The essential and fundamental factor in the evolution of a real monkey is the development of a macula lutea and all that this involves in a series of profound changes in the nervous system, and an even more striking influence on the animal's behavior and capabilities." He cites Woollard (1925), who studied the retina of Tarsius spectrum, and who found there what must be considered as an area centralis retinae - a local thickened area with an increase in the number of visual elements. Elliott-Smith speaks of this as a primordium maculae and contrasts it with the true fovea such as occurs in a primitive monkey, e.g. the marmoset. He regards the development of the true macula as intimately associated with the altered position of the eyes and the extensive overlapping of the visual fields. The position of the eyes has already changed in Tarsius, but according to him the rearrangement of the optic tracts in this creature does not seem to have attained a stage that is essential before the macula can develop. He says further, "Before this important 
change can take place in the retina all the fibers coming from the temporal side of the retina should remain uncrossed for the macular area develops in the line where the temporal and the nasal fields of the retina meet, and one-half of it transmits fibers which remain uncrossed, and the other half transmits fibers which cross to the other side of the brain. Hence until the rearrangement of the fibers in the optic chiasm has been completed the true macula cannot develop. Intimately linked with this process of evolution is the development of a wide range and a greater exactitude in the conjugate movements of the eyes."

One is left somewhat in doubt as to what is implied by the "true macula." Speaking of mammalian retinas Duke-Elder $(1939$, p. 101) says, " . . . only in some monkeys and man is there a true macula with a fovea subserving binocular and stereoscopic vision." If the true macula implies binocular vision, then it cannot be confined to anthropoids and man, for in many birds the temporal macula is supposed to subserve the function of binocular vision. In the owl the visual axes are almost parallel (Figure 3). Wolff (1940) also makes the statement: "Only man and some monkeys have a true macula and fovea centralis subserving binocular and stereoscopic vision." In discussing the bird retina he speaks of an area with a fovea, although Casey Wood (1917) describes this avian region as a macula and Duke-Elder also refers to it as a macula.

The macular region in anthropoids and man is the region of highest visual acuity where the layers of the retina are spread apart, so as to produce a depression known as the fovea. The displacement of the retinal layers at the fovea produces a thickening in the parafoveal region. The structural conformation is exactly the same in principle in anthropoids as it is in diurnal saurians regardless of the difference in shape between the foveas of these two groups of animals.

Duke-Elder defines the macular region in man as follows, "The region of the retina at the posterior pole of the eye is specially differentiated to subserve the highest visual acuity. 
To bring this about the cerebral layers of the retina are spread apart and the blood vessels which accompany them are lacking so that the light can travel without hindrance to the receptive elements; in this way the optical disadvantages of an inverted retina are neutralized. Moreover, the neuro-epithelium itself becomes eminently specialized and contains cones only." He divides this region into three concentric rings, viz.,

1) The macula lutea - characterized by yellow pigment.

2) The rod-free area within the above, containing cones only.

3) The fovea centralis (central pit) in the center.

Wolff (op. cit.) defines the macula lutea as follows: " . . a shallow oval depression about the same size as the disc. Its center lies $3.5 \mathrm{~mm}$. lateral to the edge of the disc and just below its middle. The side wall of the depression is called the Clivus, and slopes gradually towards the fovea centralis." He says further, "It will thus be seen that at the fovea centralis the layers of the retina are spread aside, so that light may fall directly on the true percipient elements, namely the cones." Since no maculae are described without the presence of a fovea centralis, it is difficult to understand the importance which is attached to this structure in anthropoids and man as a true macula. The same essential configuration occurs in reptiles and birds. Are we to call this an 'untrue' or pseudo-macula and fovea? There seems to be no apparent reason for speaking of this as a true macula and fovea in monkeys and man just because it happens to be associated with such features as partial optic decussation, binocular vision, and conjugate eye movements.

The area centralis retinae is typically a localized thickening of the retina produced by crowding of the visual elements. It is usually circular and is present in some amphibia and in many reptiles (Figure 62). It may be in the form of a band or stripe as in the alligator (Chievitz, 1891). The typical area does not have a central pit or fovea. 
Both Woollard and G. Elliott-Smith have emphasized the existence of the so-called area centralis retinae in the eye of Tarsius as a forerunner to the development of the anthropoid macula and fovea. With respect to this region Woollard says: "This differentiated area lies on the lateral side of the entrance of the optic nerve, that is in the position of a macula, and represents the macula of Tarsius. It is possible to arrange in a series the transformations taking place in this region of the retina for the enhancement of vision. The first degree shows a gradual increase in all the layers of the retina. The second a well-defined area in which this increase takes place. Finally, an area in which a fovea is formed and only cones are present, the other layers being pushed aside in order not to interfere with the transmission of light. Tarsius overlaps the first and second in having a well-defined area in which the layers have increased. The cones, however, are absent and a fovea is not formed." The difficulty of accepting Woollard's findings and interpretations is based upon two main facts. First, this differentiated area centralis, according to him, consists of a series of localized thickened convolutions in the retina. If these localized convolutions or folds which he describes in Tarsius represent a real area centralis, it is difficult to understand how such a region could function for increased visual acuity. A marked localized increase in the number of retinal elements in the visual axis of the eye does provide for enhanced visual acuity, such as in the turtle, but it would seem that the existence of retinal convolutions or undulations in such a region could do no other than bring about a marked distortion of the image in the same way that a wavy photographic plate would destroy definition. Secondly, since Tarsius is strictly a nocturnal animal possessing rods only, it seems questionable that a real area centralis should be present on these grounds alone. In so far as I am aware, areas and foveas do not exist in strictly nocturnal forms.

In my own studies upon the nocturnal lemurs (Galago and Nycticebus) I have observed retinal convolutions or folds, 
but they are not localized and are interpreted as due to shrinkage resulting from technical procedures. It would seem impossible to think of a localized convolution of the retina as a region for increased visual acuity, representing the forerunner of the macula and fovea.

The development of foveas (and they are always associated with macular areas as generally defined) has no absolute dependence upon any particular arrangement of opticus fibers. It is true that in humans, the fovea develops in the line where the temporal and nasal fibers of the retina meet (the nasal fibers crossing - the temporal fibers remaining uncrossed). However, a very prominent fovea is present in diurnal lacertilians where there is complete crossing of the optic tracts, and in many salt-water fishes where there is also complete decussation, there is present a fovea lateralis. How could Elliott-Smith's interpretation apply to birds where in many forms there are two maculae and two foveae? The opticus fibers, even were they both crossed and uncrossed, could not possibly meet in two widely separated places. Regardless of the position of the eyes in birds, optic decussation in these forms is also complete (Harris, 1904). Amphibia with complete optic decussation lack a fovea. It is apparent, therefore, that the development of this structure is not necessarily bound up with any particular arrangement of crossed and uncrossed opticus fibers.

According to Harris (op. cit.) optic decussation is complete in all fishes, amphibia, reptiles, and birds, whether they possess binocular vision or not. He regards binocular vision as originally associated with carnivorous habits - pointing out its existence in a few sharks and rays, in toads, and in many carnivorous birds (e.g. the larger gulls, some penguins, hawks, owls, and vultures). Among mammals it is especially developed in carnivora and in the primates. Thus while many animals below the higher mammals enjoy a fair degree of binocular vision, their optic nerves show complete decussation and their vision is thus not stereoscopic in the same sense as in higher mammals, anthropoids, and man, where 
visual impressions from both eyes are recorded on the same side of the brain, by reason of partial optic decussation.

In discussing the great variations in eye positions among birds in relation to binocular vision Harris (op. cit.) says: "Although the vision of a pigeon and of an owl is so different in character, the one having laterally placed eyes, an extended range of vision on both sides, and no binocular vision, while the latter has the eyes placed right forward, with accurate binocular vision, but with a less extended range, yet the decussation of the optic nerves is complete in both cases."

Among mammals, there is also considerable variation in the position of the eyes. In the herbivora and rodents they are laterally placed; in the carnivora and in primates they are forward, and these animals possess a high degree of binocular vision. The rabbit, for example, with its eyes placed quite laterally, and with practically no binocular vision, does exhibit some uncrossed fibers (Singer and Münzer, 1889), a condition which stands in marked contrast with the laterally placed eyes and periscopic vision of many lower vertebrates wherein the optic decussation is complete. Apparently the decussation is complete in mice and rats (Singer and Münzer, op. cit.).

That the development of a wide range and a greater exactitude in conjugate movements is intimately linked with macular and foveal development, as implied by ElliottSmith, also does not necessarily seem to follow. The chameleon, although he may enjoy temporary conjugate eye movements and binocular vision, exhibits strikingly independent movements of the eyes and probably for the most part has only monocular vision. This is certainly well illustrated in slow motion pictures of the chameleon's activities which are at the author's disposal.

It would seem from the studies of Kahmann (1936) that marked motility of the eyes is associated with foveal development, but this does not necessarily imply the existence of binocular vision. In 21 marine teleosts (representing 10 families) which possess well-developed eye movements, he 
found a fovea lateralis (Figure 79), and these forms exhibit definite monocular vision. The absence of the fovea lateralis in fresh-water fishes is apparently associated with a lack of eye muscle movements. In forms higher than the fishes, the fovea lateralis (temporalis), wherever it exists, presumably involves binocular vision. Some snakes are described as possessing a lateral fovea, and reference has already been made to their presence in certain rapacious and other birds (e.g. hawks, swallow, and tern).

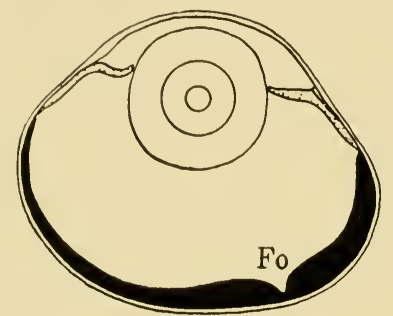

A

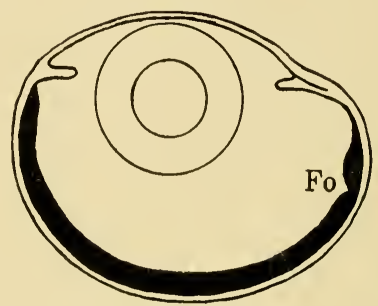

B

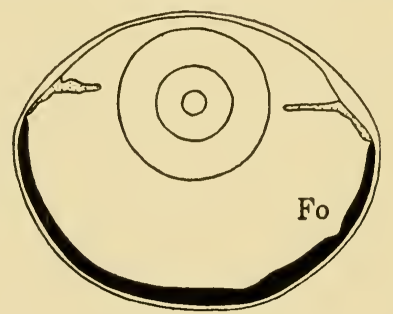

$\mathrm{C}$

FIg. 79. Diagrams showing fovea lateralis in three salt-water fishes. a, Hippocampus guttulatus; b, Serranus scriba; c, Coris julius. Fo, fovea lateralis. (Photographed from Kahmann, 1936. v. Graefe's Arch.f. Ophth., Bd. 135)

Whereas it is seen that foveal development and the ability for marked movements of the eyes seem to go together, conjugate movements, binocular vision, and partial decussation of the optic tracts are not necessarily implied. In this connection Kahmann points out that the presence of a forea in so many animals, among which a binocular use of the eyes is never made, shows us how much monocular vision predominates. 
Elliott-Smith also attributes profound effects in cortical development to macular vision. In this connection he says: "In the case of the tarsier and the marmoset, two creatures not very dissimilar in size, the brain of the monkey is much bigger, perhaps as much as five times the weight of that of Tarsius. The chief reason for this contrast is the development of the macula and in association with it the large number of new fibers which pour into the lateral geniculate body and through it stimulate a series of profound changes in the neopallium."

If the development of the macula is responsible for the greatly increased size of the marmoset brain over that of Tarsius, then might we not expect profound changes in the diurnal lacertilian brain as compared with that of the chelonians, for the lacertilians possess highly developed foveas with more elements per unit area than in the human fovea. Might we not also expect similar evolutionary advances in the brain of those birds possessing a highly developed macular area with a fovea, or especially in those containing two macular areas. To be sure, in the forms below the mammals there are no optic radiations and also no neopallium to stimulate, but even here the greatly increased number of fibers pouring into the primary optic centers from the fovea should produce an equivalent effect upon these centers connected with the primary optic stations - if the assumed principle of stimulation is valid. Both in the fovea-bearing lizards and birds, the numbers of ganglion cells in the region of the macula are increased just as they are in anthropoids and man, therefore, the augmentation of ingrowing fibers to the brain has the same chance of producing profound advances in the brain of these forms as obtains in the marmoset or any other monkey. Experiments designed to give evidence pro or con on the alleged stimulating effect of ingrowing opticus fibers upon proliferation in the lateral geniculate body, and in turn upon the cortex, have been undertaken by my colleague, Doctor Chace. His results (in press) show that unilateral removal 
of the rat eye at birth is followed by a hypoplastic development of the corresponding external geniculate body. The effect was not discernible until about one month after the enucleation.

Woollard (1927), who described the rod retina of the owl monkey, Nyctipithecus, the only true monkey of strictly nocturnal habits, says: "Nyctipithecus is no exception to the rule that a true macula and fovea are only found when cones form the chief constituent of the sensory epithelium of the retina. These observations add support to the conception that Nyctipithecus is the most primitive of the Platyrrhines." The first part of this statement is unquestionably true, but it is also true that the lack of cones and a fovea can in no manner be used as an argument in support of the thesis that Nyctipithecus is the most primitive of the Platyrrhines. Some authorities (personal interviews) regard the marmoset as a more primitive form than Nyctipithecus, yet its retina not only predominates in cones, but it possesses a typical anthropoid fovea (Figure 64). Neither the presence nor absence of a macula and fovea can be used to pigeonhole any animal in the evolutionary scale. Eyes with pure rod retinas are specializations and are correlated with nocturnal life. Those with pure cone retinas are adaptations for diurnal vision at high intensities of light. Foveas constitute mechanisms for increased visual acuity, be they present in fishes, lizards, birds, monkeys, or man. Pure rod retinas are adaptations for nocturnal vision whether they occur in lizards, bats, lemurs, or monkeys.

With regard to the functional significance of the fovea, a stimulating theory has been advanced by Walls (1937). It has been generally regarded that the attenuation or displacement of the retinal layers at the fovea presents a condition whereby light may reach the foveal cones unimpeded, i.e. without passing through the ordinarily present nuclear layers of the retina. This idea has become quite firmly fixed and is stressed in textbooks of ophthalmology. It necessitates the assumption, of course, that the transparency of 
the retina is less than that of the vitreous, an assumption which Walls questions. He justly calls attention to the fact that in an afoveate area centralis, the retina is thicker than the less specialized retina beyond its limits; yet, in spite of this, the resolving power of the area centralis retinae is obviously much greater, indicating therefore that a thin spot per se is apparently not so important. In comparing the profile of the highly developed fovea of diurnal saurians with that of man, he makes a distinction between the two types, that of the saurians being convexiclivate - that of man, concaviclivate. He regards the shallow fovea as seen in such forms as the woodpecker, pigeon, fowl, and owls as a mark of foveal degeneracy brought about by nocturnality or domestication.
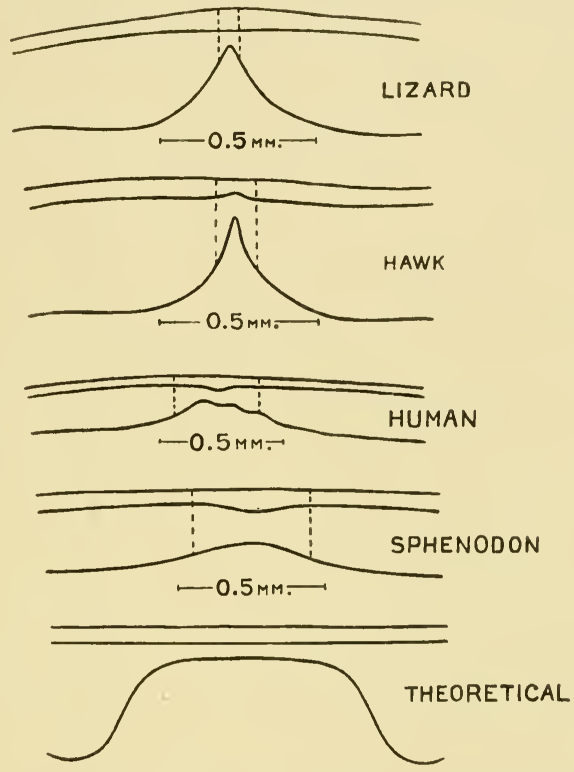

FIG. 80. Outline drawings of the fovea centralis from various retinae. (Redrawn from Walls, 1937. Arch. Ophth., v. 18.) See text for explanation of theoretical fovea.

Similarly, the human fovea is regarded as 'crude' - either never having developed to a high degree or else being degenerate. He argues further, that if removal of tissue were desirable per se, then the concaviclivate form such as in man, Sphenodon, and the poor-sighted birds would be superior to the convexiclivate (sharp-sighted) forms of the diurnal saurians, and these forms, upon such a basis, should have a profile similar to the theoretical one shown in Figure 80. Discussing then the theory that the foveal depression has developed merely to remove tissue, so that light can fall unimpeded upon the visual cells, he produces 
reasons for regarding the limiting membrane of the foveal clivus as constituting a refractive surface. The internal limiting membrane cannot differ markedly from the rest of the retina in refractive power or there would be a condition detrimental to vision. He regards the general retinal tissue as differing from the vitreous in refractive index. If the retina had a lower refractive index, total reflection in the deeper part of a convexiclivate fovea would render the central portion of such a fovea blind. Going originally on the

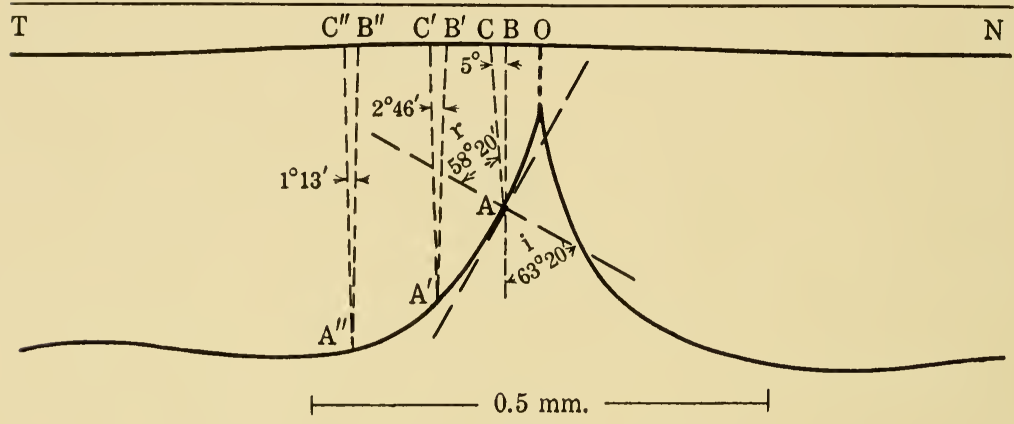

FIG. 81. Convexiclivate fovea of Saurians to illustrate refraction of light along the foveal clivus and spreading of the image on the foveal photoreceptors, according to Walls. (Figure redrawn from Walls, 1937. Arch.Ophth., v. 18.)

assumption that the retina has a higher refractive index than the vitreous, Walls produced logical reasons in support of the idea that the foveal clivus refracts the light, thus broadening the retinal image and bringing into play a larger number of visual cells than would otherwise be the case (Figure 81). By reason of this property there thus occurs at the fovea a mechanism for increasing the resolving power over that produced by the afoveate area centralis. Using a theoretical example, employing certain assumptions, Walls figured the refractive index of the retina to be about 1.403 as compared with 1.336 for the vitreous. He also stressed the point that even in the concaviclivate fovea of man where the refractive index may not be so high as 1.4 , there should still be an expansion of the image, although such a form optically would be inferior to that of reptiles and birds. 
Subsequent to the postulation of this theory, Walls (1940) found and presented a record of measurements of refractive indices of a number of vertebrates made by Valentin (1879). The most important figures are those of a foveate parrot (Chrysotus aestiva). Valentin gave the figure 1.3475 for the refractive index of the retina and 1.3391 for the vitreous. By substituting these actual numbers for his own hypothetical ones, Walls (op. cit.) says that angle $B A C$ (Figure 81) proves to be $1.64^{\circ}$, which affords an expansion of the image in the middle of the hawk's fovea of approximately 30 per cent. Discussing matters further from his findings, he says: "In the concaviclivate human fovea, however, the much less precipitous clival slope and the presumptive lower refractive index would appear to make for a degree of image expansion too low to account for the evolution of the human foveal pit from no depression at all directly to its present gentle profile. Wherever the foveas of animals approach the fovea of man in shallowness, they can invariably be shown to have been deeper in the ancestors. In the light of the resurrected data of Valentin, it seems more likely than ever that the human fovea has degenerated, like that of Sphenodon, the owls and the pigeon, from a once much more deep and abrupt depression. The possibility that the higher apes have less gradual foveas than man seems fairly strong, and it is to be hoped that studies of them may soon be made."

If the theory of Walls proves to be the correct one, and there is strong evidence in its support, then the concept of the fovea as a region devised to admit light unimpeded to the photoreceptors must be discarded, and this localized region of specialization must be viewed as a mechanism for increasing the resolving power of the retina. It has the distinct merit of supporting the generally recognized fact that the foveas of reptiles and birds are more efficient mechanisms for increased visual acuity than those of anthropoids and man. It remains, of course, to see whether this concept will stand the test of examination from physiological and purely optical viewpoints. 


\section{CHAPTER $X$}

\section{RETINAL PHOTOPIGMENTS}

Both rod and cone vision depend upon the decomposition by light of a photosensitive substance which occurs in the outer segments of the visual cells. Since the substance is affected by visible light, it obviously absorbs visible light and is, therefore, a pigment. It is apparent also that the photopigment when broken down must be resynthesized or vision would cease soon after irradiation had begun. The stimulating product or products, as well, must be removed rapidly or vision would continue long after irradiation has ceased.

In connection with rod function the photopigment rhodopsin (visual purple) has long been known. This photosensitive substance was discovered in the rods of frogs by Franz Boll in 1876. It is a rose-colored substance and was termed visual purple or rhodopsin by Kühne (1878) who with Ewald studied it in much detail, as did many subsequent investigators. ${ }^{1}$ Koenig (1894) first showed the similarity of the visibility curve at low illuminations and the curve representing the absorption spectrum of visual purple (Figure 82). The obvious inference to be derived is that visual purple is concerned with vision at low light intensities. Many added physiological facts have completely supported the early-formed impressions. As to the origin of this pigment, many diverse opinions have been elaborated. Ayres and Kühne (1878) maintained that there is a relation between epithelial pigment and the regeneration of visual purple. They compared the epithelial pigment cell to a gland, the secretory product of which is visual purple. This idea was supported by experiments in which the use of pilocarpin

${ }^{1}$ For a recent discussion of the photochemistry of visual purple, v. Krause, A. C., Biol. Symposia, v. 7, p. 23. 1942. 
was found to reduce the time of visual purple regeneration in the dog and rabbit. Kühne (1879) cited the parallelism between the time needed for regeneration of visual purple in the frog and the length of time in which dark adaptation is first accomplished as evidence of a relationship between the two.

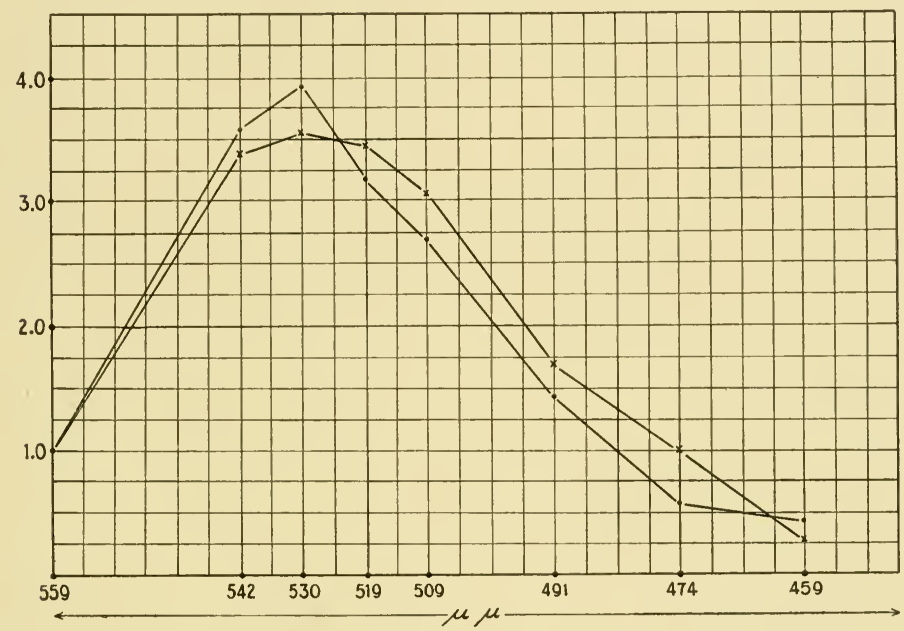

FIG. 82. Showing the similarity between the curve representing the rate of bleaching of visual purple by light of different wave lengths and the luminosity curve of twilight vision. (From Starling, Human Physiology, 7th Edition. Lea \& Febiger, Philadelphia.)

Garten (1907) suggested that it was not the epithelial pigment that was concerned with the generation of visual purple, but the basal part of the pigment cell itself. This was obvious from the fact that visual purple was found to occur in the pigment-free portions of the retina in many animals and in the eyes of albinos.

When a dark-adapted retina is exposed to light, its rose color quickly fades to a yellowish or orange color and finally to white. If it is again placed in the dark, it will regenerate its original pink or rose color. On this matter there have been differences of opinion, for some observers claimed that the pigment will regenerate only if the retina is accompanied 
by the pigment epithelium, thereby favoring the idea that the pigment cell is responsible for its generation. Although Kühne (op. cit.) reported that visual purple in solution will regenerate some of its color in the dark, this observation has apparently never been confirmed until recently by Hecht (1936) and his co-workers. The properties of rhodopsin in solution have been examined recently by Wald (1938) in marine fishes, frogs, and mammals. He showed that the bleaching of neutral rhodopsin in solution includes a photic and at least three thermal ("dark") processes. Thermal reactions account for approximately half of the total fall in extinction at $500 \mathrm{~m} \mu$. He showed also that the spectrum of pure rhodopsin corresponds in form and position with the spectral sensitivity of human rod vision, computed at the retinal surface.

Boll (op. cit.) observed that, in frogs, the pigment epithelium firmly adheres to the retina when exposed to light thus implying some functional relation between this tissue and the visual purple. The carotenoid nature of visual purple has been studied extensively by Wald (1935b, 1936), who has shown also that the pigment epithelium contains large stores of vitamin A and xanthophyll esters. Since the presence of vitamin $\mathrm{A}$ is now well known to be essential to the production of visual purple, it becomes apparent that the storage of this substance in the pigment epithelium firmly establishes an important relation between this cellular layer and the retina proper. Light liberates from the visual purple a carotenoid (retinene) which by a reaction independent of light (thermal) is converted to vitamin A. Vitamin A and retinene are also the precursors of visual purple. The visual processes, therefore, constitute a cycle (p. 125).

It is interesting in this connection to cite the early formulated photochemical theory of Hecht (1920, 1927), who studied extensively the photochemistry of light responses in invertebrates and in the human retina. He was led to the conclusion that the photosensitive mechanism is composed of two processes. The initial one has the character- 
istics of a photochemical reaction, while the subsequent one is an ordinary thermal reaction which is catalyzed by the substance formed in the first one. In the first reaction the photosensitive substance $\mathrm{S}$ is decomposed by light into the products $\mathrm{P}, \mathrm{A}$, and $\mathrm{B}$. This reaction is pseudo-reversible; in the dark or light, $\mathrm{P}$ and $\mathrm{A}$ recombine in the presence of a new material $\mathrm{C}$, present in excess, to form the original photosensitive substance. The process in its simplest form, therefore, was written by Hecht as follows:

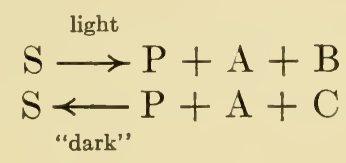

In this scheme the substance B acts to initiate the sensory impulse. The chemistry of the rhodopsin system as since analyzed by Wald agrees in its general properties with this scheme. This in itself is a marked tribute to the logical excellence of these pioneer researches of Hecht.

Visual purple, since its discovery, has been known to be an unstable substance decomposed by light and it was originally hoped that this substance would become the basis of an allinclusive photochemical theory of vision. However, its reported complete absence in many animals lacking rods, as well as its absence at the fovea where rods are absent, soon made it clear that the existence of other substances must be recognized in order to account for the physiology of cone vision. Hecht and Williams (1922), however, studied the visibility curves at high and at low intensities of light and found their absorption spectra to be so close as to suggest the presence of visual purple in dilute solution in the cones. Logical reasons based upon the findings of Weigert (1921) were given to support their contention that both cone and rod vision might be accomplished by a single photosensitive substance. This idea in so far as I am aware has since been abandoned, and cone vision is accomplished apparently by the presence of a different photopigment. 
In fact even rod vision has been shown to be mediated by slightly different photopigments.

Fridericia and Holm (1925) and Tansley (1931) showed that animals deprived of vitamin A become abnormally insensitive to dim light (night blind), due to the failure to synthesize visual purple. In recent years the problem of vitamin A deficiency and raised visual thresholds has become one upon which many investigations have been launched.

The condition of night blindness has been known for ages and its relation to poor nutrition recognized. It was seen in men returning from long sea voyages, in people living in prisons, and among Orthodox Russians during Lenten fasts. The use of liver in its treatment has been widespread. According to Tansley (1931) the earliest reference appears in Eber's Papyrus dated about 1500 B.c. Liver treatment was apparently recommended by Hippocrates.

Some of the most extensive experimental work dealing with the relation of vitamin $\mathrm{A}$ to vision has been done by Wald, who has shown that vitamin $\mathrm{A}$ is the precursor of visual purple as well as the product of its decomposition. He has worked extensively upon frogs and fishes.

Rhodopsin bleaches in light and is resynthesized in the dark. In aqueous solution its absorption spectrum consists of a broad band, maximal at $500 \mathrm{~m} \mu$. It has the properties of a protein, but it is a conjugated protein, the special properties of which involve principally a colored prosthetic group. This is derived from the widely distributed class of yellow to red, highly saturated, lipoidal pigments known as the carotenoids (Wald, 1935b). Dark-adapted retinas, owing to their content of rhodopsin, are rose-colored. On irradiation they bleach to an orange color. Wald (1938) has shown that this reaction in solution consists of a succession of light and 'dark' (thermal) processes, the latter accounting for at least half the total change in spectrum. The orange-colored substance in neutral fat solvents yields a yellow lipoid pigment which he has called retinene and which is a carotenoid. In aqueous solution, retinene with a protein are the final 
products of bleaching. In the isolated retina the orange color fades and the tissue becomes colorless. The extracts of completely faded retinas are also colorless. They contain no retinene, but a large quantity of newly formed vitamin A, the final product of bleaching. The same results are achieved by prolonged illumination of the intact retina. In the living animal placed back in darkness, vitamin A is resynthesized to rhodopsin. The retinal cycle is thus represented by Wald (1938, 1939a, 1939b) as follows:

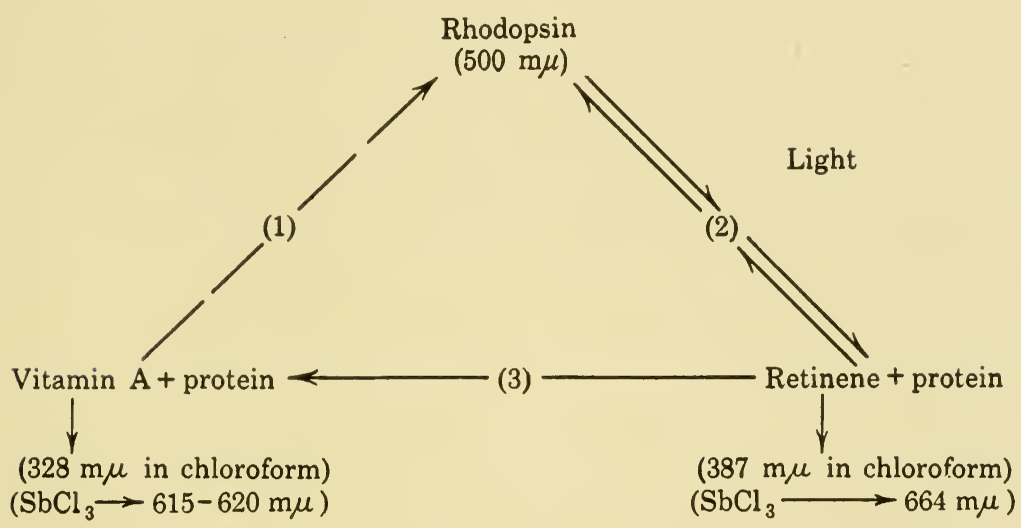

In isolated retinae the cycle is cut at (1) so that vitamin $\mathrm{A}$ is the final product of bleaching. When rhodopsin is extracted into aqueous solution, reactions (2) and (3) are also eliminated, leaving only the succession of light and dark processes which form retinene.

Kühne and Sewall (1880) noted that the dark-adapted fish retinas which they studied were unlike those of all other vertebrates in that they were purple-colored. Köttgen and Abelsdorff (1896) confirmed this difference spectrophotometrically. This purple photopigment was called porphyropsin by Wald. Although it was thought for many years that the fishes as a class possess the purple pigment porphyropsin, an examination of the retinas of a number of marine fishes, by Wald (1936-1937), showed them to possess typical rhodopsin cycles, and showed further that por- 
phyropsin is peculiarly characteristic of fresh-water fishes alone. The absorption spectra of rhodopsin from the rabbit, bullfrog, four marine fishes, and a typical porphyropsin absorption spectrum from the white perch are given in Figure 83 (Wald, 1938).

Porphyropsin in aqueous solution possesses also the properties of a protein. Its spectrum consists of a broad band, maximal at $522 \mathrm{~m} \mu$. Under illumination it bleaches in a succession of light and 'dark' (thermal) reactions to a

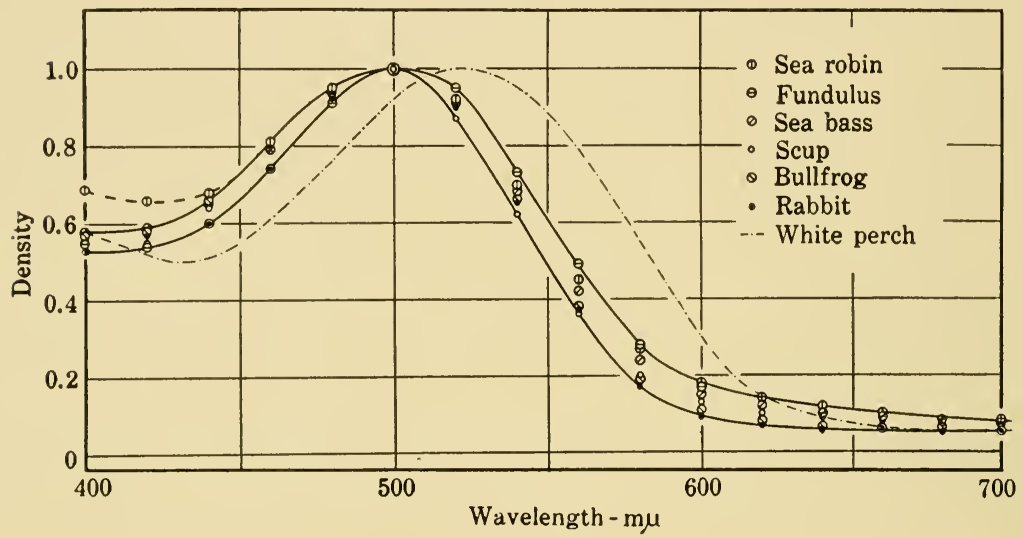

FIG. 83. Spectra of rhodopsin preparations from the rabbit, bullfrog, and four marine fishes; and of a typical porphyropsin preparation from the freshwater white perch. (From Wald, 1938. J. Gen. Physiol., v. 21.)

russet color, due to a carotenoid pigment. This colored product has been termed by Wald, retinene $e_{2}$, which in the retina is transformed further to a new pale yellow carotenoid yielding with antimony chloride a band maximal at $696 \mathrm{~m} \mu$. This has been designated vitamin $\mathrm{A}_{2}$ in contrast with the vitamin $A_{1}$ of the rhodopsin system which gives a band at 615-620 $\mathrm{m} \mu$ in antimony chloride. Porphyropsin was found to participate in a retinal cycle identical in form with that of rhodopsin but in which a $706 \mathrm{~m} \mu$ chromogen $\left(\right.$ retinene $_{2}$ ) replaces retinene and a $696 \mathrm{~m} \mu$ - chromogen (vitamin $\mathrm{A}_{2}$ ) replaces vitamin $\mathrm{A}_{1}$ (Wald, 1937a, 1939a, 1939b). 
The porphyropsin cycle was thus formulated by Wald as follows:

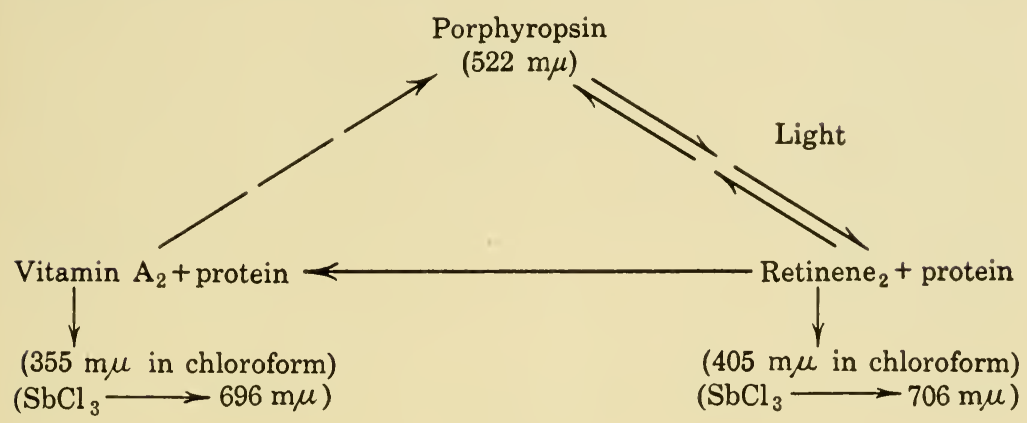

It is thus seen that the performance of the two systems is identical in detail, but a constant difference in spectrum divides all components in one cycle from their analogues in the other.

The difference between the two systems apparently lies in the fact that throughout the porphyropsin system there has been added one double bond in the carotenoid chain. This is known from spectrophotometric studies on a series of synthetic carotenoids to shift the spectrum 20-30 $\mathrm{m} \mu$ towards the red. The separation between the vitamin $A_{1}$ and $\mathrm{A}_{2}$ maxima is $27 \mathrm{~m} \mu$; between rhodopsin and porphyrop$\sin , 22 \mathrm{~m} \mu$; and between retinene ${ }_{1}$ and retinene ${ }_{2}, 18 \mathrm{~m} \mu$. The differences are in satisfactory agreement with the proposed structure. It appears, therefore, that the only chemical difference between the two visual systems is the possession by the porphyropsin system of one added double bond. Wald found that all the fresh water fishes which he examined possess only porphyropsin systems, but he also discovered that those fishes which spawn in fresh water and live normally in the sea (anadromous) possess primarily the porphyropsin systems, whereas those which live mostly in fresh water but spawn in the sea (catadromous) possess primarily the rhodopsin systems. These so-called euryhaline fishes, which are capable of existence in both environments, thus possess either predominately or exclusively that photopig- 
ment and that vitamin A ordinarily associated with the environment in which the fish is spawned. For example, the anadromous white perch possesses porphyropsin only, yet its close marine relative, the black sea bass, possesses only rhodopsin. Likewise, the anadromous alewife contains only porphyropsin, but its close relative, the permanently marine herring contains rhodopsin exclusively. The anadromous brook trout, rainbow trout, and chinook salmon (all salmonids) were found to contain mixtures of rhodopsin and porphyropsin - predominately the latter. In the catadromous eel and killifish, however, which contain both pigments, rhodopsin was found to predominate. It became obvious from these findings that the pattern of retinal vitamin A is not primarily an environmental response, but is fixed genetically, and its significance is to be sought in the phylogeny of fishes.

Originally, having found vitamin $A_{2}$ only in fresh water and anadromous teleosts, Wald (1939b) proposed that this vitamin may represent a comparatively recent evolutionary development, associated with the re-migration of marine fishes into fresh water. More recently, however, Wald $(1942 a, 1942 b)$ has found the porphyropsin system to predominate also in the anadromous lamprey, a cyclostome and one of the most primitive of living vertebrates. He concluded that the association of vitamin $\mathrm{A}_{2}$ with the capacity for fresh water existence is, therefore, a very general and primitive property, at least among vertebrates.

In addition to these important findings regarding the rod visual systems, Wald (1937b) also partially isolated from the chick a cone photopigment which he designated iodopsin. This was accomplished by irradiating chicken retinal extracts with deep red light, a method which has long been known to stimulate the cones but not the rods and rhodopsin. The initial bleaching which was obtained by red light was believed to be due to the photopigment of the cones. The bleaching properties showed it to be a violet pigment (hence the name iodopsin), with an absorption 

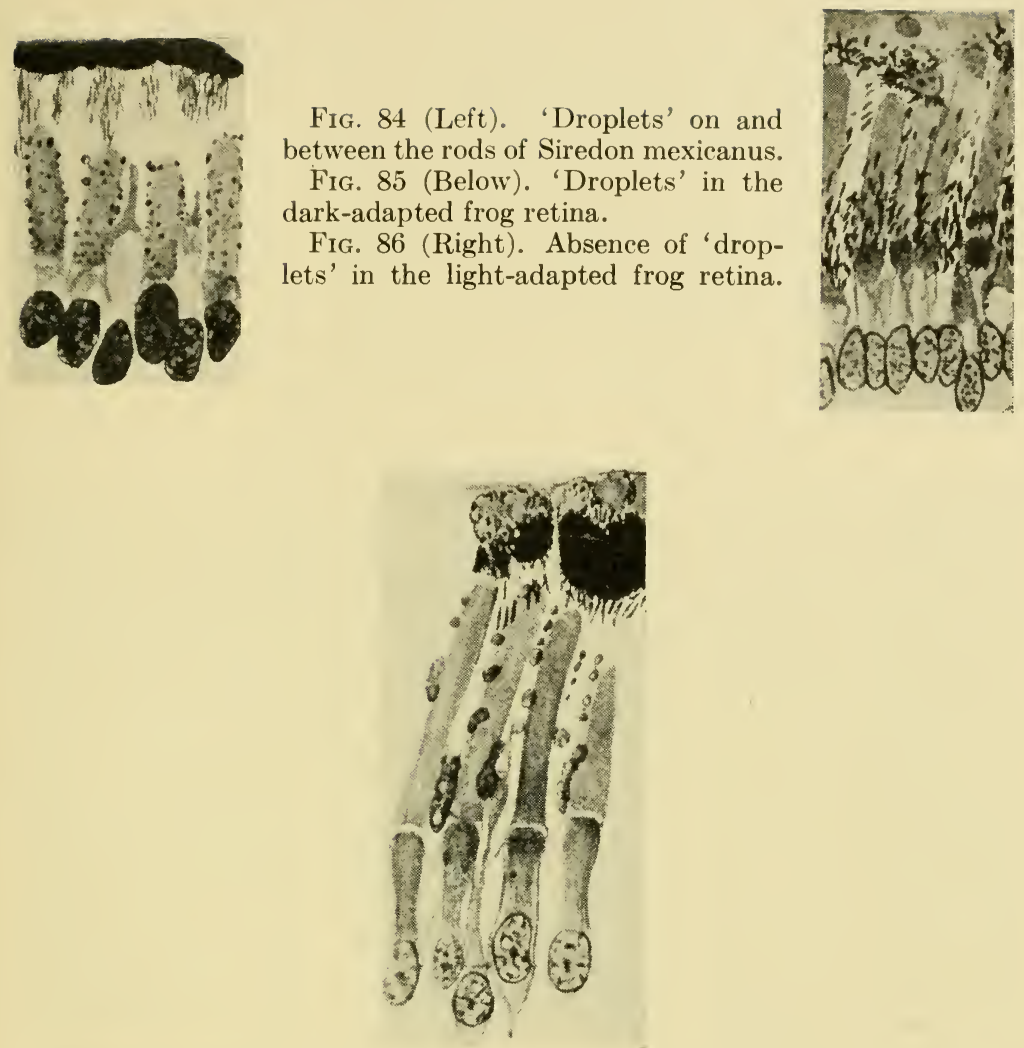
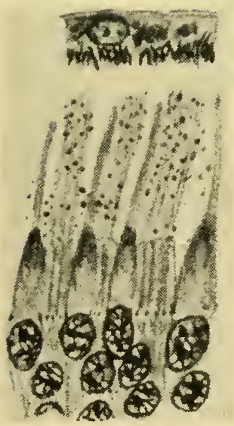

Fig. 84 (Left). 'Droplets' on and between the rods of Siredon mexicanus.

FIG. 85 (Below). 'Droplets' in the dark-adapted frog retina.

FIG. 86 (Right). Absence of 'droplets' in the light-adapted frog retina.
Fig. 87 (Left). 'Droplets' in the retina of Macacus rhesus.

Fig. 88 (Right). 'Droplets' in the retina of the goose.

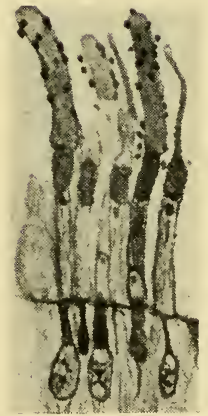

Figures 84 to 88 . Preparations showing 'droplets.' All photographed from Kolmer, 1909, Arch.f. d. ges. Physiol., Bd. 129. All figures from Table 2. 
maximum about $575 \mathrm{~m} \mu$ in contrast with that of rhodopsin whose maximum is about $500 \mathrm{~m} \mu$.

It can be said that up to the present time only three retinal photopigments have been identified, viz., rhodopsin and porphyropsin, which are rod photopigments, and iodopsin, which is a cone photopigment. All vertebrate visual systems have been built upon the carotenoid pigments. The synthesis of these pigments, as far as is known, depends upon the presence in the diet of a carotenoid in the form of a vitamin. Vitamin A bears this relation to rod vision in most animals and where deficiencies arise, night blindness results.

Whenever one finds marked physiological or chemical changes taking place in a system one naturally seeks for some histological evidence of these changes. In this connection might be cited, among other findings, the so-called Kolmer's 'droplets.' In 1909 Kolmer found that when the retina was preserved with a dichromate fixative containing acetic acid and stained subsequently with iron hematoxylin, there occurred on and between the visual cells deeply staining granules or 'droplets' (Figures 84-88). In dark-adapted retinae these granules were abundant, whereas in lightadapted retinae they were sparse. Since the administration of pilocarpine brought about an increase, even in lightadapted eyes, he stressed their secretory nature and their origin from the pigment epithelium.

Although Kolmer originally was inclined to regard the droplets as artifacts, he finally concluded that they were secretory products of the pigment epithelial layer. His observations led him to point out several correlations between the droplets and visual purple, viz., 1) the presence of droplets on the outer segments or on the processes of the epithelial pigment cells, the latter regarded at that time as the source of visual purple; 2) their absence in pure cone retinae of diurnal lizards in which visual purple is absent; 3) their abundance in dark-adapted retinae containing rods, wherein visual purple is also at its maximum; 4) their sparsity in light-adapted retinae, in which visual purple is absent or 
present in small quantities; and 5) the effect of pilocarpine on the number of droplets - an effect similar to that reported by Ayres and Kühne (1878) upon the production of visual purple.

In the gecko (Detwiler, 1923c) which has rods only, the droplets were found to be abundant on the rod outer segments in dark-adapted retinae and sparse on the external limbs following light-adaptation (Figure 89) (v. also Figure 90).
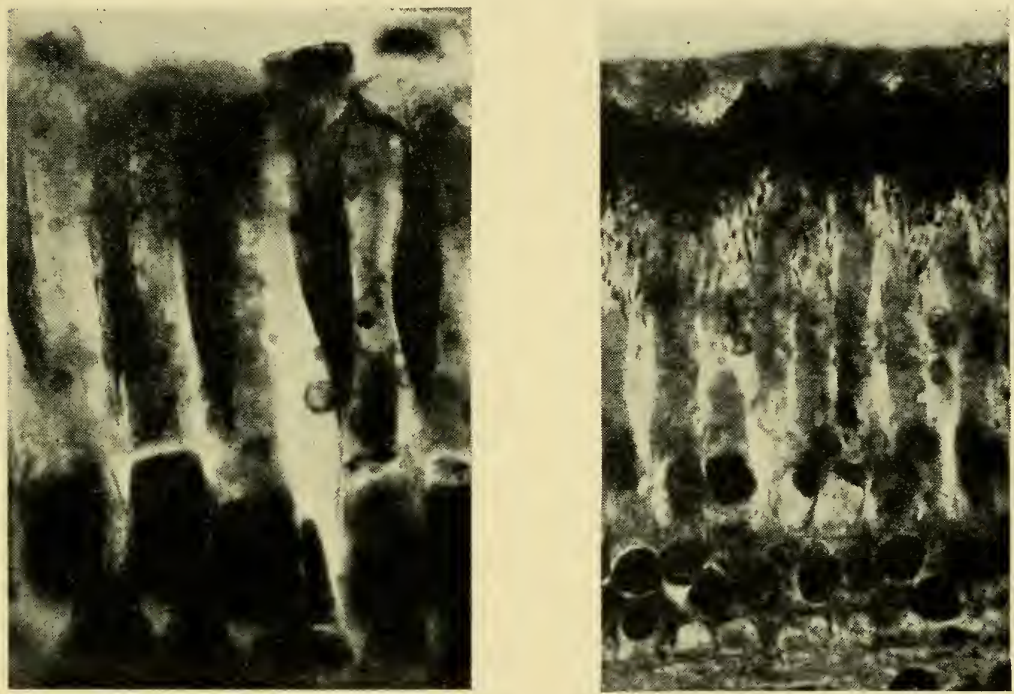

FIG. 89 (Left). Photomicrograph showing 'droplets' on and between the rods of the dark-adapted retina of the gecko. $\times 900$.

FIG. 90 (Right). Photomicrograph showing 'droplets' on and between the rods of the dark-adapted frog retina. $\times 630$.

It was noted also that in light-adapted eyes, where the droplets were few in number, the rod outer segments were abundantly filled with a dark staining granular material which appeared to have a lamellar arrangement. In the dark-adapted eyes, with abundant droplets, the rod outer segments contained very little of this deeply staining material. Since sections from both dark and light eyes were mounted 
together and stained on the same slide, this difference indicated that the droplets were related in some way to the rod outer segment rather than to the pigment epithelial cells. This view was later adopted by Kolmer (1925) who then claimed to have demonstrated their presence among the foveal cones of the chimpanzee. The rod origin of the droplets was substantiated by Keeler (1927) who was unable to demonstrate their presence in the retinae of mice exhibiting an hereditary absence of rods, although the pigment epithelial layer was present and normal in appearance. Further proof of the rod origin of these structures was produced by Johnson (1935) who demonstrated their presence in the grafted retinae of Amblystoma punctatum which lacked completely an epithelial pigment layer.

In the developing rat retina (Detwiler, 1932) it was shown that stained droplets in fixed retinae were not demonstrable until after visual purple was definitely visible in the fresh dark-adapted retina. The fresh retina could be recognized as pink at twelve days after birth, at which time the rods in fixed eyes exhibited granular material within the outer segments, but no droplets outside the rod were seen in fixed preparations until fourteen to eighteen days (v. Detwiler, op. cit., p. 487).

In 1939 Johnson reported that the droplets are not present in the retinae of rats suffering from severe avitaminosis A. Their absence under these conditions added further indirect evidence that these structures bear some close relationship to visual purple or some phase of its cycle, since Tansley (1933a) has shown that visual purple is also incapable of being formed in the retinae of rats suffering from a lack of vitamin $\mathrm{A}$.

Except for the work of Walls (1934b, 1939a), who regards the droplets as artifacts, bearing no relation to the visual process, all of the aforementioned observations seemed to point to some relationship of the droplets to the visual cycle, but offered no evidence as to the nature of the droplets nor of the relationship between them and rhodopsin. Evidence 
was later produced (Detwiler and Zwemer, 1937) which demonstrated that the droplets are lipoidal, staining similarly to cephalin. Kolmer (1925) also suggested their lipoidal nature.

Wald $(1935 a, 1935 b)$ showed that visual purple, when exposed to light, breaks down into retinene (visual yellow) which in turn bleaches further, at room temperature, to form certain colorless products, one of which has been identified as vitamin A. In darkness, at room temperature, retinene recombines to form visual purple. Both retinene and vitamin A are typical lipoids, soluble in organic solvents. Having determined the lipoidal nature of the droplets, we (Detwiler and Zwemer, op. cit.) intimated a possible relation between the droplets, retinene, and vitamin $\mathrm{A}$, rather than with visual purple itself, which has been shown by Wald (1935b), and by Hecht, Chase, and Shlaer (1937) to have the nature of a conjugated protein (a carotenoid protein).

Based upon a careful study of Wald's (1935a, 1935b, 1936, 1938) analyses of the visual purple cycle, Johnson (1939) suggested that the droplets might actually be retinene.

Recently we (Johnson and Detwiler, 1942) have shown that, whereas the droplets bear at least one property in common with each of the major components of the visual cycle (visual purple, retinene, and vitamin A), they coincide in all respects with only one of these substances, viz., retinene (Table 3). The droplets and vitamin A are both lipoidal and both are absent in extreme vitamin A-deficient animals, but the droplets are most prominent in dark-adapted retinae, whereas vitamin $\mathrm{A}$ can be demonstrated in the retina proper only after light-adaptation. Further, the droplets and visual purple show similar behavior under different conditions of illumination, and are both absent in extreme avitaminosis A (Johnson, 1939, Tansley, 1933a), but they differ in essential structure and in mode of concentration. The droplets are lipoidal and demonstrable only in retinae fixed with bichromate-acetic acid solution or in platinum chloride. Visual purple is a conjugated protein 


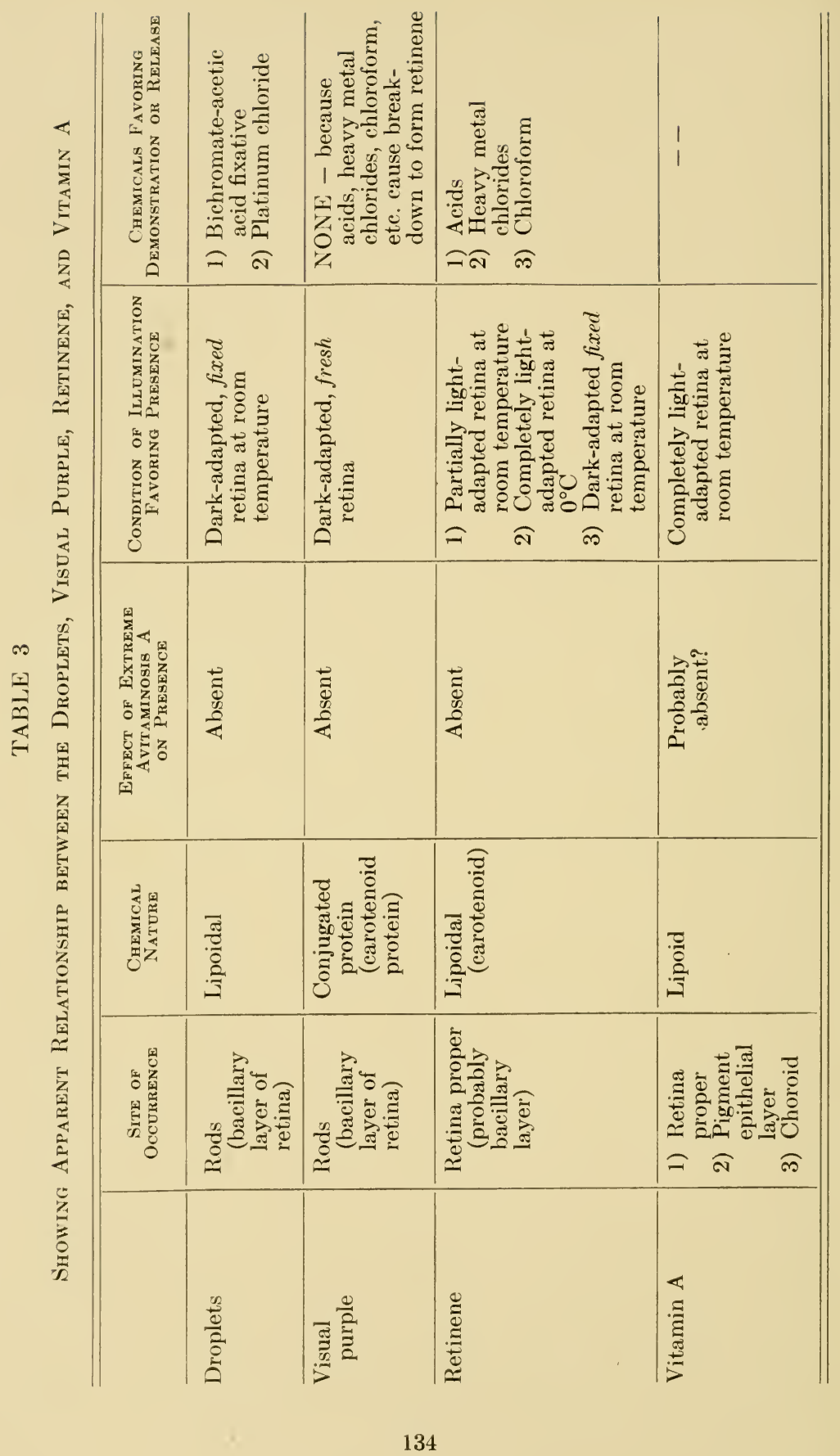


which is converted to retinene and protein when treated with acids and heavy metal chlorides (Wald, 1935a, 1935b).

The droplets and retinene, however, not only have a common (rod) origin and lipoidal structure, but both are known to occur under similar conditions, viz., in retinae dark-adapted at room temperature and treated with certain acids and heavy metal chlorides. Wald (op. cit.) has shown that retinene is liberated in the isolated fresh retina (or in solution), through the break-down of visual purple on brief exposure to light at room temperature. It is present in light-adapted fresh retinae at $0^{\circ} \mathrm{C}$, at which low temperature the final bleaching of retinene to vitamin $\mathrm{A}$ and protein is inhibited. It is demonstrable also in large quantities in either isolated dark-adapted retinae (or in visual purple solution) when they have been treated with acids, heavy metal chlorides, or chloroform.

Since Wald $(193 \tilde{b} b, 1936,1938)$ then demonstrated that the conversion of retinene to vitamin $\mathrm{A}$ or its reconversion to visual purple is a thermal reaction which is inhibited at low temperatures, we (Johnson and Detwiler, op. cit.) endeavored to test further the validity of our theory that the droplets may be retinene by determining whether they (the droplets) exhibit a behavior similar to that of retinene under like conditions of temperature and illumination. This involved experiments to determine 1) whether the droplets remain demonstrable in the retinae of animals light-adapted at $0^{\circ} \mathrm{C}$, after having been dark-adapted at room temperature, and 2) whether the droplets are either incapable of demonstration or sparse in the retinae of animals dark-adapted at $0^{\circ} \mathrm{C}$ (following light-adaptation at room temperature).

Using frogs (Rana pipiens) à series of nine experiments was performed in which the animals were subjected to various conditions of temperature and illumination (Table 4 , columns 1 and 2). In each case it was known from Wald's (op. cit.) studies on the isolated fresh retina what materials predominate in both fresh and fixed retinae under given conditions (Table 4, columns 3 and 4). It was known from 
previous work on simple dark-and light-adaptation at room temperature that the droplets are typically numerous in dark-adapted eyes and sparse in light-adapted ones. According to Wald's findings, retinae dark-adapted at room temperature are rich in visual purple, but after treatment with acids, the visual purple is converted to retinene. Under these conditions droplets should be numerous. Further, after prolonged exposure to light, visual purple is bleached to vitamin $A$ and protein, but with traces of retinene and visual purple. Under these conditions the droplets should be few or lacking. Experiments 1, 2, 6, and 8 (Table 4), consisting of light-and dark-adaptations, at room temperature (Figures 91 and 94, 93 and 96), have supported earlier findings (Kolmer, 1909, and Detwiler, 1923c). The experiments involving dark- and light-adaptations at $0^{\circ} \mathrm{C}$ yielded results which accord with the theory that the droplets exhibit a behavior parallel to that of retinene under various coriditions of temperature and illumination (Experiments 3, 4, 5, 7, and 9 , Table 4).

Animals were light-adapted by placing them for at least one hour or longer under two 60-watt lamps, after which the eyes were excised and fixed in modified Held's solution (Kolmer's bichromate-acetic acid solution). Animals were dark-adapted at room temperature by leaving them in total darkness for four hours (some cases over night), after which the eyes were excised under faint red light (Wratten filter, series 2), and fixed in modified Held's solution in the dark.

When animals were subjected to low temperatures, they were first 'adapted' at room temperature to the desired illumination, and were then placed in a dish of water, which was kept at $0^{\circ}-0.5^{\circ} \mathrm{C}$ by immersing it in another dish filled with cracked ice and salt - the required conditions of illumination being maintained. At this temperature of the water, the oesophageal temperature of the frogs never exceeded $1.5^{\circ} \mathrm{C}$.

The eyes under all conditions of illumination and temperature were fixed alike (modified Held's), cut at $8 \mu$ and stained 
Figures 91 to 100 inclusive are photomicrographs of the frog visual cells and epithelial pigment layer under various experimental conditions as indicated. $\times 515$. All are reproduced from an article by Johnson and Detwiler, 1942, J. Exp. Zool., v. 89.
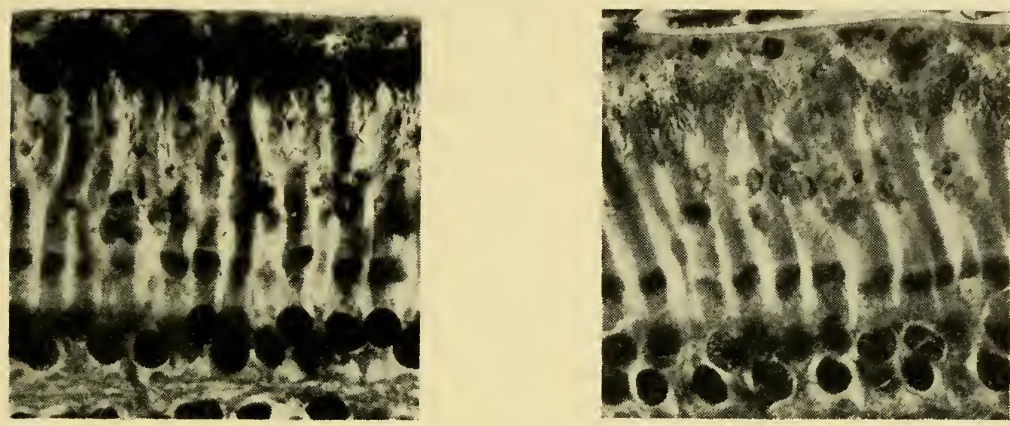

FIg. 91 (Left). Frog, dark-adapted over night at room temperature. Retina shows presence of numerous droplets and incomplete contraction of the epithelial pigment.

FIG. 92 (Right). Frog, dark-adapted over night at room temperature and then for two additional hours at $0^{\circ} \mathrm{C}$ in the dark. Retina shows presence of numerous droplets and almost complete contraction of the epithelial pigment.
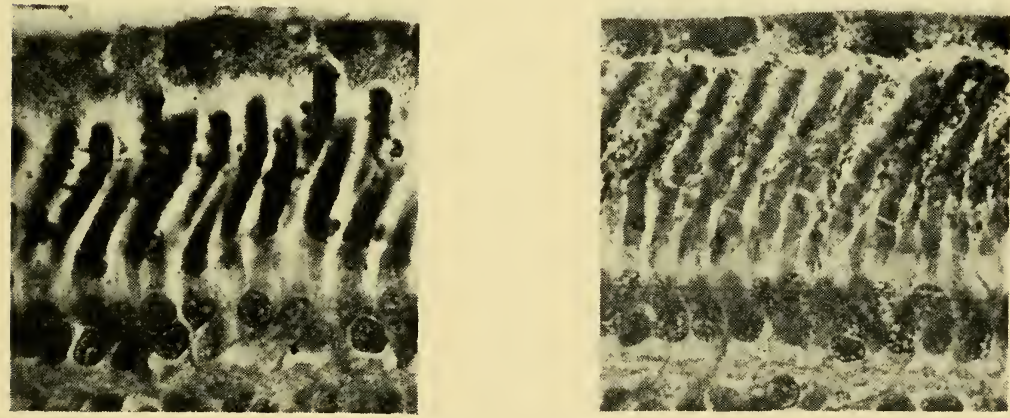

FIg. 93 (Left). Frog, light-adapted for one hour at room temperature, followed by dark-adaptation at room temperature. Retina shows fully contracted pigment and presence of numeroùs droplets.

FIG. 94 (Right). Frog, light-adapted at room temperature. Retina shows sparsity of droplets and marked migration of the epithelial pigment.

with iron hematoxylin and erythrosin. Some sections from each eye were depigmented with 10 per cent oxalic acid and 1 per cent potassium permanganate. Eyes from animals which were dark-adapted at room temperature, then cooled 

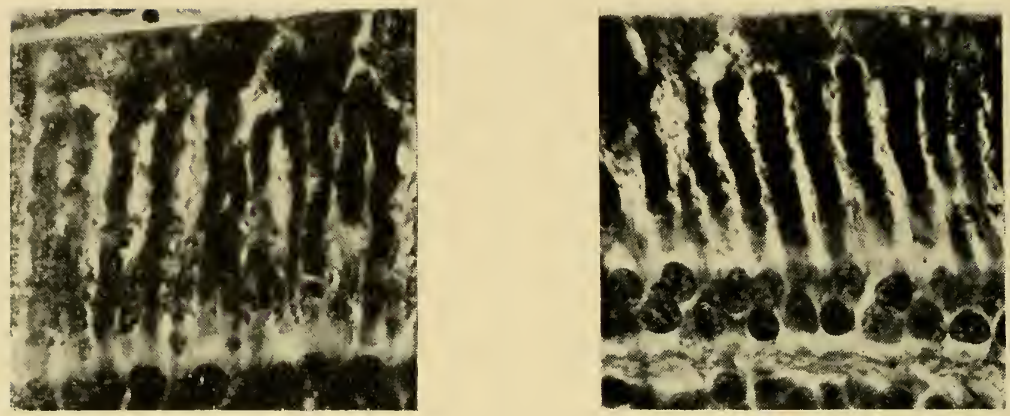

Fig. 95 (Left). Frog, light-adapted at room temperature and then at $0^{\circ} \mathrm{C}$ for one hour. Retina possesses few droplets and extensive pigment migration.

Fig. 96 (Right). Frog, dark-adapted over night at room temperature, followed by light-adaptation for one hour at room temperature. Retina shows extensive pigment migration and very few droplets.
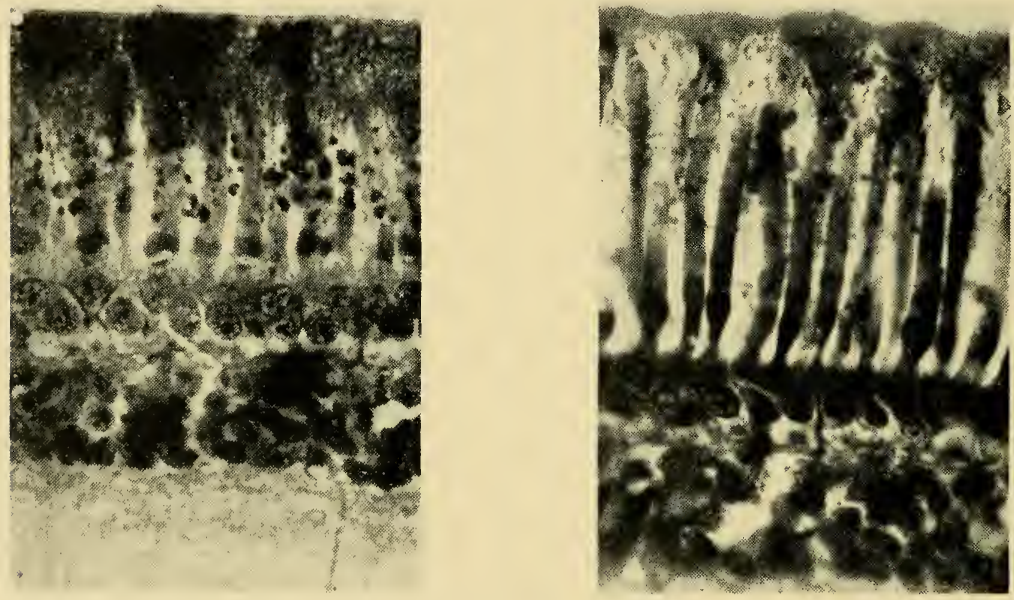

FIG. 97 (Left). Frog, dark-adapted over night at room temperature, then dark-adapted for one hour at $0^{\circ} \mathrm{C}$. Retina shows partly contracted pigment and the presence of abundant droplets.

FIg. 98 (Right). Frog, dark-adapted over night at room temperature, then dark-adapted for one hour at $0^{\circ} \mathrm{C}$, and finally light-adapted for one hour at $0^{\circ} \mathrm{C}$. The histological picture is similar to that in experiment 4 (figure 97) in showing partially migrated pigment and numerous droplets.

to $0^{\circ} \mathrm{C}$ for one hour in the dark, with subsequent illumination at $0^{\circ} \mathrm{C}$, exhibited the conditions characteristic of dark-adaptation. The epithelial pigment was partially contracted and the droplets were abundant (Table 4, Experiments $4 \mathrm{~A}$ and 

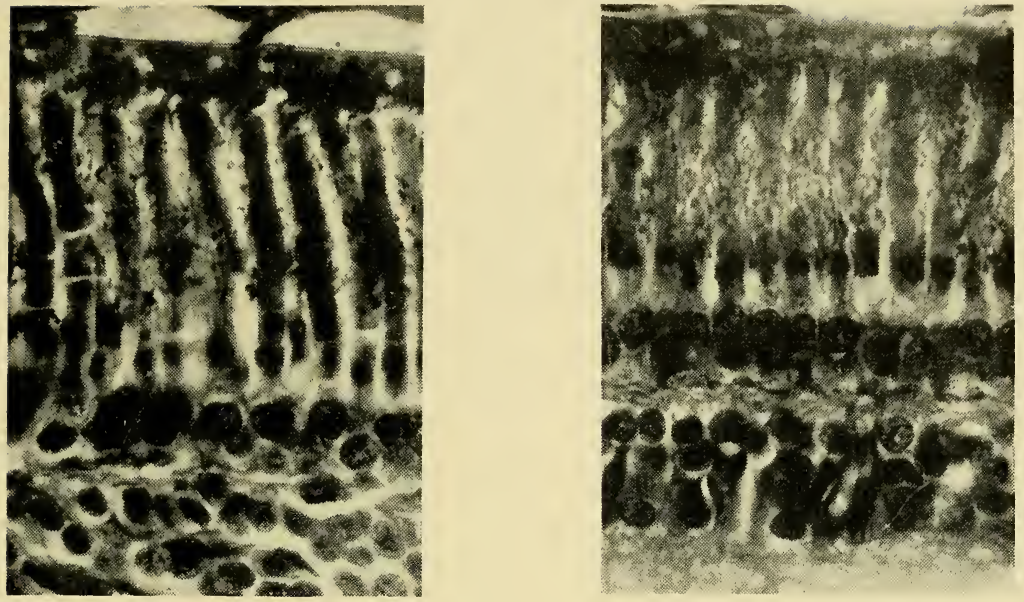

Frg. 99 (Left). Frog, dark-adapted at room temperature, dark-adapted one hour at $0^{\circ} \mathrm{C}$, light-adapted one hour at $0^{\circ} \mathrm{C}$, then allowed to come back to room temperature in the light for two hours. The retina shows sparsity of droplets and almost maximal migration of the epithelial pigment.

Frg. 100 (Right). Frog, light-adapted at room temperature, then at $0^{\circ} \mathrm{C}$ for one hour, and then dark-adapted for one hour at $0^{\circ} \mathrm{C}$. The retina shows sparsity of droplets and maximal migration of epithelial pigment.

B, 5R: and Figures 97, 98, cf. Figure 92). If, after treatment as indicated above, the frogs were allowed to come to room temperature in the light (Experiment 5L, Table 4) the eye in section exhibited conditions typical of light-adaptation at room temperature, viz., fully expanded epithelial pigment and a sparsity of droplets. The results are shown in Table 4 (Experiment 5L) and in Figure 99. The failure of the epithelial pigment to contract in the dark at $0^{\circ} \mathrm{C}$ is in line with the former observations of Herzog (1905), Arey (1916a), and Detwiler and Lewis (1926) which showed that at low temperatures, in the dark, the epithelial pigment tends to migrate as it does in the light.

The converse of the above conditions is illustrated by Experiment 7 (Table 4). Here the frog was first lightadapted at room temperature, then brought to $0^{\circ} \mathrm{C}$ in the light for one hour, and then placed in darkness at $0^{\circ}$. Eyes so treated exhibited conditions more characteristic of lightthan of dark-adaptation. The droplets were sparse and 


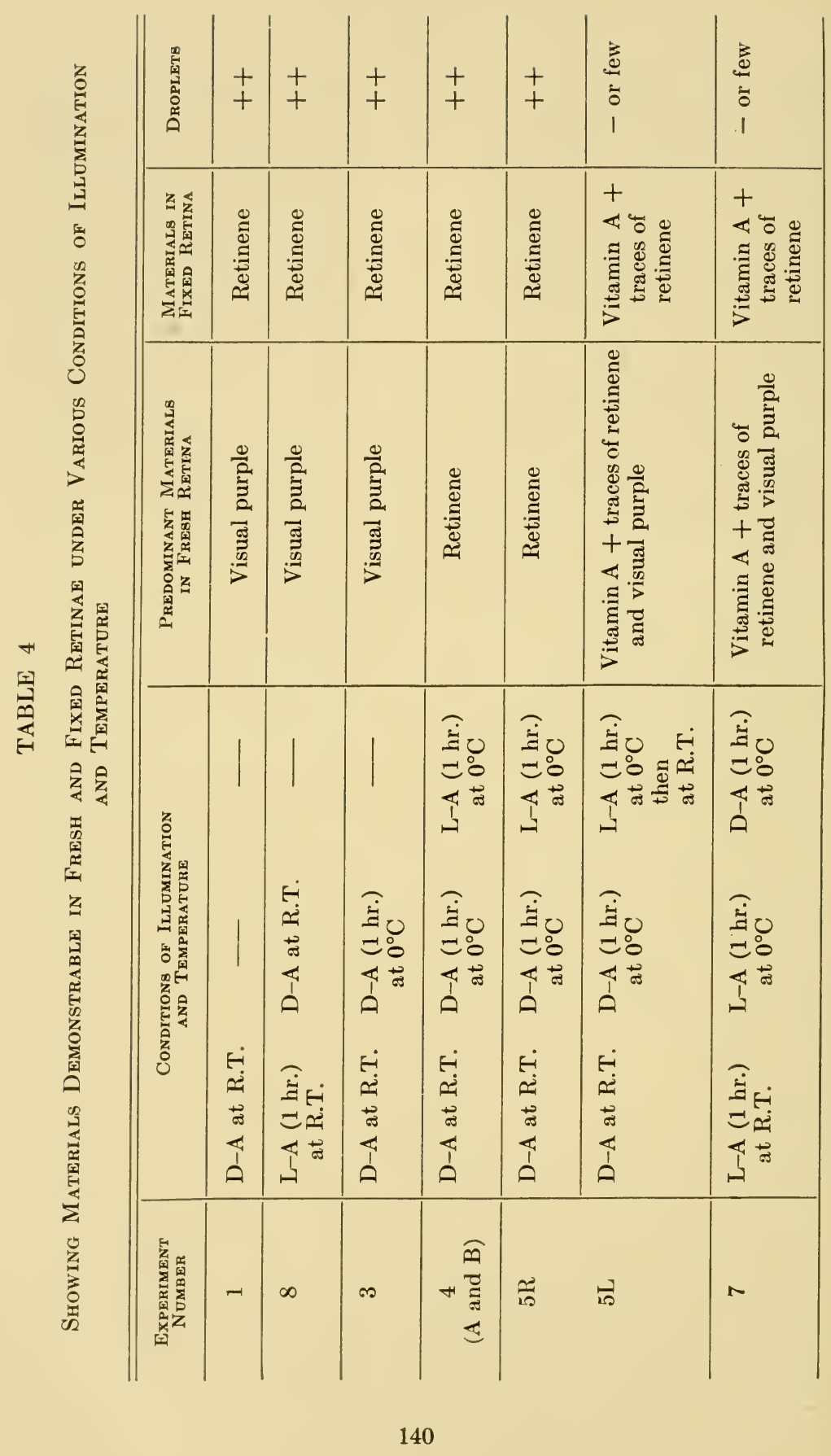




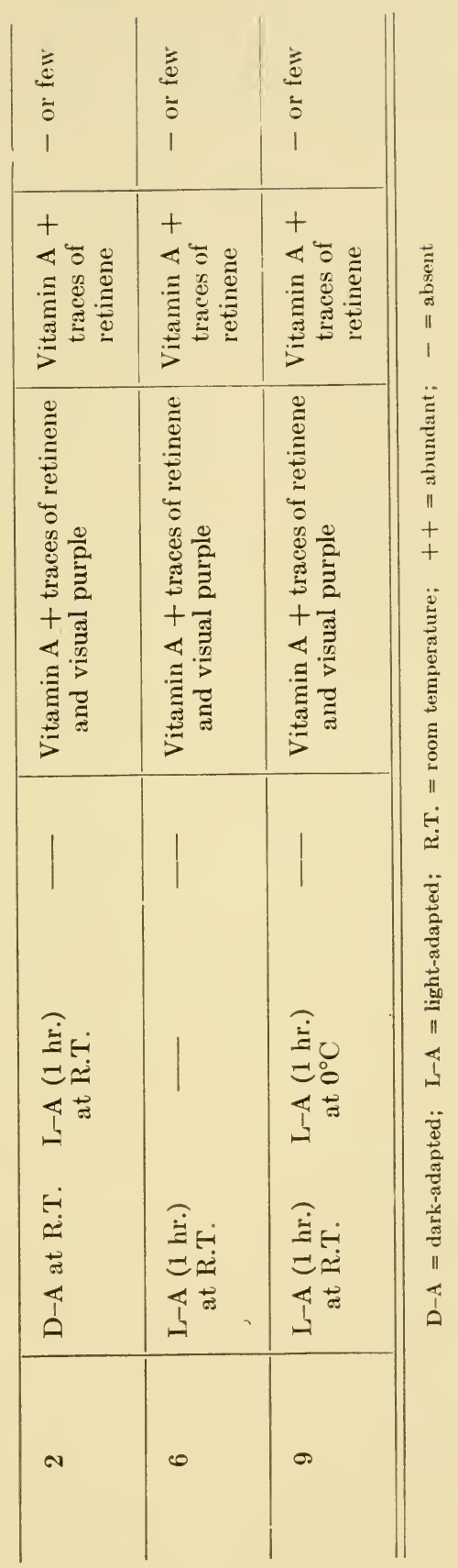


there was extensive migration of epithelial pigment (Figure 100, cf. Figure 95).

The results of our experiments on the whole have shown that droplets are usually abundantly present in frog retinae, light-adapted at $0^{\circ} \mathrm{C}$ (following dark-adaptation at room temperature), and that they are present in small numbers in retinae dark-adapted at $0^{\circ} \mathrm{C}$ (following light-adaptation at room temperature). The results thus strengthen the theory that the droplets may represent a histological picture of retinene. The droplets and retinene have been shown to exhibit common properties and similarities of behavior. These may be cited as follows: 1) site of occurrence, 2) lipoid nature, 3) absence in extreme avitaminosis, 4) presence in the retinae of animals dark-adapted at room temperature and treated with certain acids and heavy metal chlorides, 5) absence or sparsity in retinae light-adapted at room temperature and treated as described above, and 6) a parallel behavior at $0^{\circ} \mathrm{C}$ under various conditions of illumination. It might be pertinent to point out here that in the light-adapted intact eyes at room temperature, retinene is converted to vitamin $\mathrm{A}$, but that two-thirds to five-sixths of the vitamin A has diffused out of the retina (Wald, 1935b, 1936). It would appear likely, therefore, that vitamin A might form droplets as readily as retinene; but it is this diffusion out of the retina that has actually removed most of the formed vitamin $\mathrm{A}$. Thus the few droplets seen in light-adapted retinas may represent this residual vitamin A, in addition to retinene, rather than retinene alone.

The results of our investigations suggest that the droplets are histological entities which may be identified with retinene; since both droplets and retinene show a striking similarity in behavior under similar conditions of illumination and temperature, and possess a number of other properties in common (v. Table 3). 


\section{CHAPTER XI}

\section{VITAMIN A-DEFICIENCY AND THE RETINA}

Visual Thresholds. Night blindness as a functional malady has been known for centuries and its relation to poor nutrition recognized. Only in recent years, however, has this relation been traced specifically to vitamin $\mathrm{A}$ in the diet, and become understandable in terms of the chemical relation between vitamin $\mathrm{A}$ and the light-sensitive pigments of the retina. Fridericia and Holm (1925) and Tansley (1931) showed that animals deprived of vitamin A synthesize visual purple more slowly than normal animals, and, in cases of extreme deprivation, may be unable to form any visual purple at all (Tansley, 1933a). In human beings night blindness has been experimentally produced by an A-deficient diet by Jeghers (1937), Hecht and Mandelbaum (1939), and Wald, Jeghers, and Arminio (1938), and the condition has also been remedied by the resumption of a normal diet. The investigations of Wald $(1935 a, 1935 b, 1936)$ have shown that vitamin $\mathrm{A}$ is an essential ingredient of the chemical make-up of the rod visual system by demonstrating that vitamin $\mathrm{A}$ is not only the precursor of rhodopsin (or of porphyropsin), but, as well, the product of its decomposition. These relations have been discussed in Chapter $\mathrm{X}$ where both the rhodopsin and porphyropsin rod visual cycles, as studied spectrophotometrically by Wald, have been given.

Not only has vitamin A-deficiency been found to raise the rod visual thresholds, but it has been shown to affect cone vision in like manner. This indicates that vitamin $\mathrm{A}$ enters into the chemical cycle of cone vision and that the cone photosensitive substance, iodopsin or visual violet (Wald, 1937b) is very likely a conjugated carotenoid protein as is rhodopsin and porphyropsin. 
With the establishment of vitamin $\mathrm{A}$ as an important component in both rod and cone visual cycles, it became apparent that visual tests might well be employed for the estimation of vitamin $\mathrm{A}$ in the body and for its detection in early stages of vitamin A deprivation, before any definite clinical symptoms of deficiency become recognizable. Such tests were made by Edmund and Clemmesen (1936) with visual intensity discrimination as an index, and by other investigators using dark-adaptation as a criterion (Jeans and Zentmire, 1934; Jeghers, 1937; Palmer and Blumberg, 1937 ; Isaacs, Jung, and Ivy, 1938; Gridgeman and Wilkinson, 1938; Hecht, 1938b; Hecht and Mandelbaum, 1939, 1940; Wald, Jeghers and Arminio, 1938; and others).

Hecht and Mandelbaum (1939) measured the dark-adaptation of one hundred and ten 'normal' persons and obtained the highest and lowest values of final threshold. The data are presented in Figure 101. Normal dark-adaptation proceeds in two steps. The first is rapid and over in a few minutes, whereas the second is late in starting and goes on for a half hour or more. The rapid first adaptation represents the behavior of the cones (Figure 101, solid circles) while the delayed and slower adaptation (open circles) represents the rod function. These authors pointed out that dark-adaptation measurements in order to be numerically precise and quantitatively valid depend upon a number of conditions as follows: 1) the intensity and 2) duration of the light present before dark-adaptation begins, 3) the area, 4) the retinal location, 5) the color, and 6) the duration of the light used for measuring the course of dark-adaptation. These six conditions were found to influence profoundly the relative extent, the duration, and the form of the two limbs of the dark-adaptation curve, as well as the time of appearance of the cone-rod transition.

Hecht and Shlaer (1938) designed an adaptometer for controlling intensities, times, positions, and areas, so that the dark-adaptation measurements were made, in all instances, under controlled conditions. 
Having established the spread in the dark-adaptation of a normal population, Hecht and Mandelbaum (op. (it.) studied the dark-adaptation of four young men on a diet with decreased vitamin A (150 units of vitamin A daily).

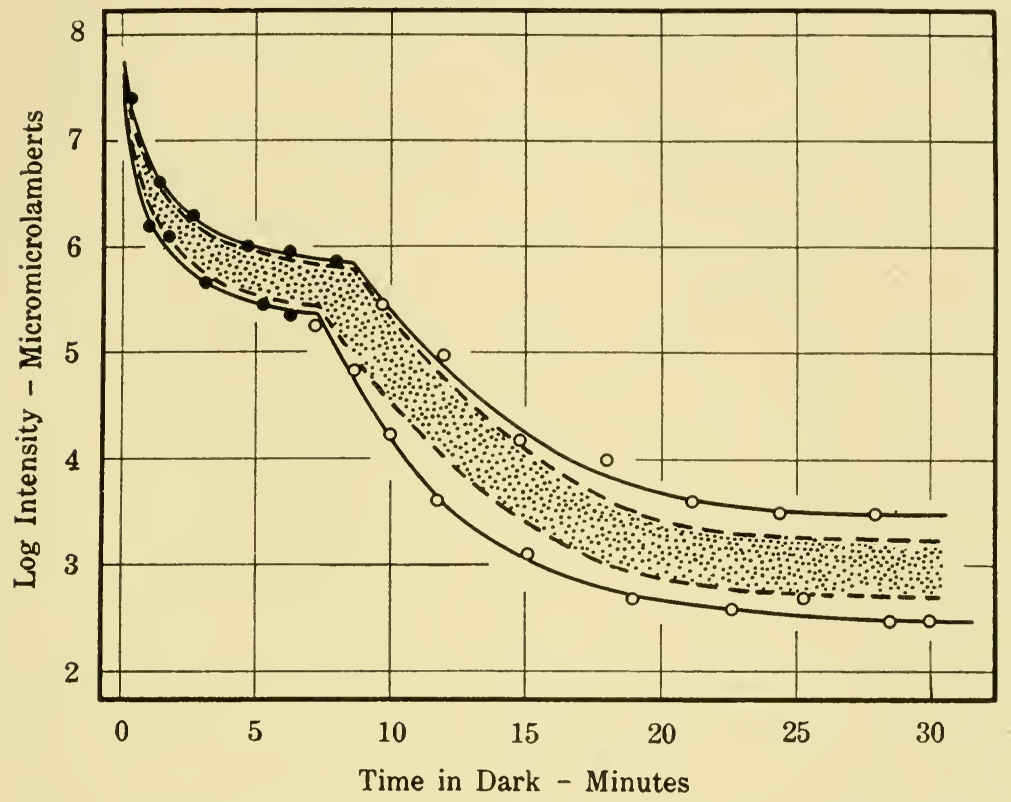

FIG. 101. Showing the course of dark-adaptation in 110 normal persons. The points represent the single measurements made with two subjects yielding the highest and lowest values of the final threshold. The dotted area contains 80 per cent of the population. The cone adaptation curve is indicated with solid circles; the rod adaptation curve by unfilled circles. (From Hecht and Mandelbaum, 1939, J.A.M.A., vol. 112.)

The diet was maintained for about forty days after which they were returned to normal food habits. Two were given daily supplements of 50,000 units of vitamin A for six weeks afterward. The dark-adaptation curves of one of these subjects with vitamin A deprivation are shown in Figure 102 where it is seen that both cone and rod thresholds are affected by the vitamin lack, and that rod vision is affected more than cone vision, as evidenced by the more rapid and extensive rise of the rod section of the curve during the diet. 
This confirmed the work of Haig, Hecht, and Patek (1938) who found more extensive changes in rod than in cone function in the dark-adaptation of patients with cirrhosis of the liver. Wald, Jeghers, and Arminio (1938) studying

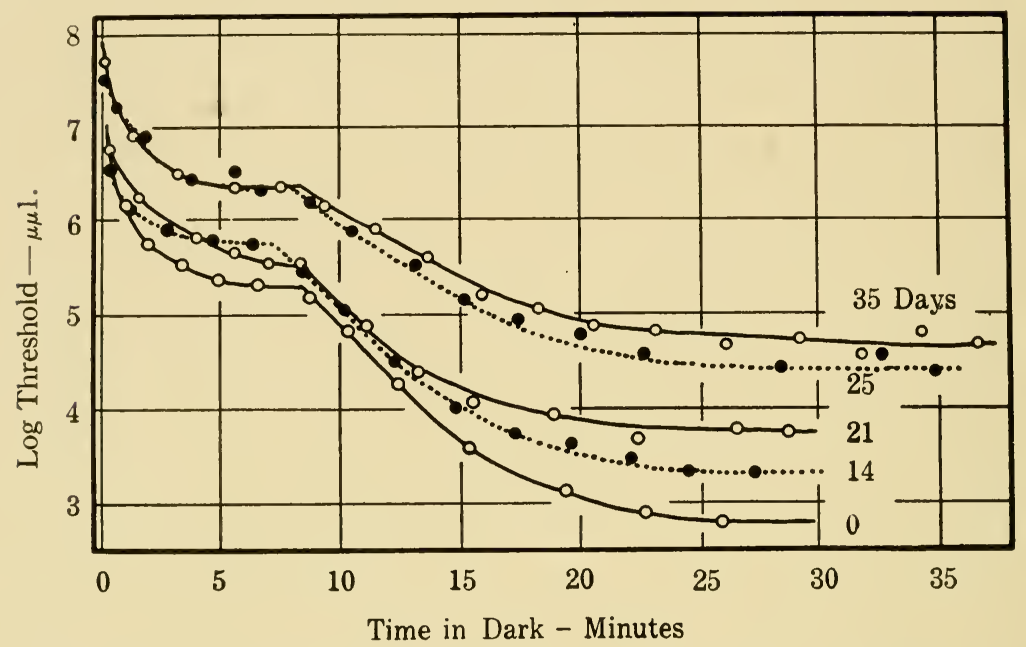

FIG. 102. Curves showing dark-adaptation measurements of a human subject made at various times during the course of a diet practically free from vitamin A. The filled and unfilled circles keep the different sets of measurements apart and are smoothed by dotted and continuous lines respectively, cf. figure 101. (From Hecht and Mandelbaum, 1939, J. A. M. A., vol. 112.)

human dietary night blindness claimed that with a deficient diet, within 25 days, the rod thresholds had risen about 50 times as compared with 5 times for the cones.

The subjects studied by Hecht and Mandelbaum (op. cit.), deprived of dietary vitamin $\mathrm{A}$, responded by a steady rise of both cone and rod thresholds, which became apparent almost from the first day after removal of vitamin A from the diet. After about two weeks' deprivation they showed thresholds above any values normally found in the population of normal individuals, and their thresholds continued to rise so long as the vitamin A deprivation was maintained (Figure 103). The return to a normal diet (with or without supplementary vitamin A) resulted in an initial small drop 


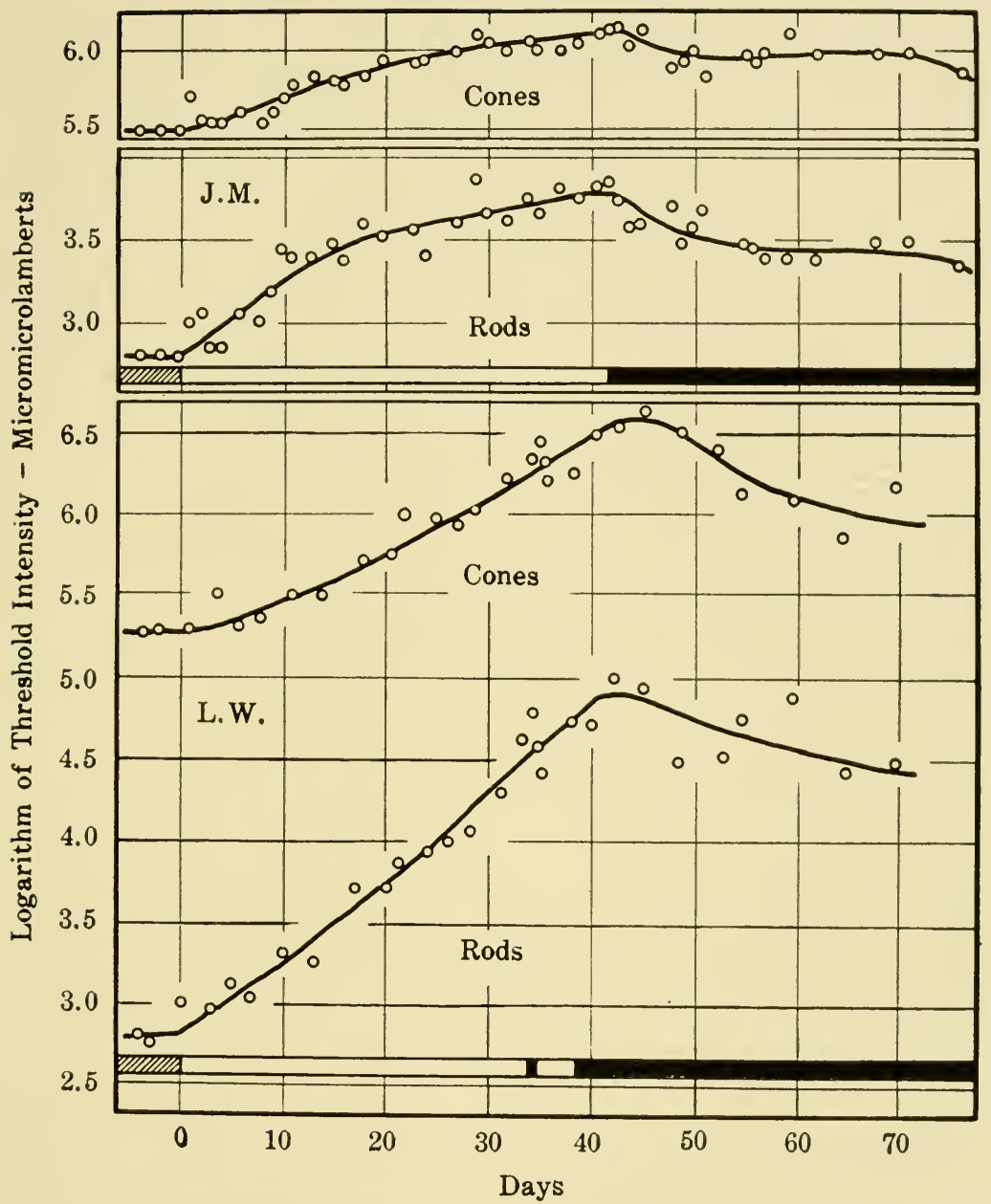

FIG. 103. Curves showing final cone and rod thresholds of two subjects on a diet nearly free from vitamin $\mathrm{A}$. The diagonally shaded part represents the normal diet, the clear part the diet free from vitamin $A$, and the black part the normal diet plus 50,000 units of vitamim A daily. (From Hecht and Mandelbaum, 1939, J. A. M. A., vol. 112.)

in threshold, followed by a gradual decrease, and nearly two months were required for the subjects to return to normal. It became apparent from these observations that the return to normal thresholds took a much longer time (even with 
supplementary vitamin A) than the time required for the maximal rise in threshold. Whereas, in some instances, the rise in visual thresholds follows almost immediately upon vitamin deprivation, there is also evidence of great variability in different individuals. Wald, Jeghers, and Arminio (1938) and Wald and Steven (1939) observed an immediate rise. Other investigators, however, did not encounter such immediate responses. Booher, Callison, and Hewston (1939) worked on five subjects, which required 16, 27, 29, 39, and 124 days respectively on a vitamin A-deficient diet before showing any recognizable rise in thresholds. Steffens, Bair, and Sheard (1939) found only slight and temporary rises in threshold for three subjects on a deficient diet for approximately six months.

Hecht and Mandelbaum (1940) worked with a group of seventeen young men placed on diets which were restricted to foods calculated to yield about 150 International Units of vitamin A daily. These were divided into three groups. The first group of four subjects received no supplements of other vitamins during the A-deficient dietary period. The second group of eleven subjects received daily supplements of 125 units each of vitamins $B_{1}$ and $G$ in the form of brewer's yeast, and 1800 units of vitamin D as irradiated ergosterol. In addition, each subject took 300 c.c. of skimmed milk daily, and was encouraged to eat grapefruit for vitamin C. The third group of two subjects received daily supplements of 200 units of $B_{1}$ and $G, 2000$ units of D, and $50 \mathrm{mgm}$. of ascorbic acid, as well as 300 c.c. of skimmed milk. These authors found an unmistakable immediate threshold rise in fourteen of the seventeen subjects. Two individuals showed hardly any rise for 60 days, and then the threshold suddenly began to rise sharply. Another subject showed an initial rise, then a drop, and fluctuated thus for 3 months before there was an unmistakable typical threshold rise. The supplementation of the A-deficient diet with other vitamins apparently had no effect upon the behavior of the thresholds. Subjects showing the most immediate rise fell 
in groups 1 and 2. Seven subjects showing similar degrees of response were distributed in all three groups. Three subjects who showed no immediate effects of the diet were all in group 2, which received adequate supplements of other vitamins. The data which these authors obtained suggest that such individuals who fail to respond for long periods of time may have an exceptional storage of vitamin $\mathrm{A}$ in the tissues which they eventually exhaust. It thus appears that storage capacity for vitamin A has no relation to amounts recently ingested. Steininger, Roberts, and Brenner (1939) found it to depend more on individual capacity and on long term history. This was supported by the observations of Wald and Steven (op. cit.), who gave their subjects a short period of high vitamin A dosage, and yet obtained widely different individual effects with a subsequent deficient diet.

Not only do the experiments show marked variations in the time required for raising the visual thresholds in subjects on a vitamin A-deficient diet, but the recovery time also has been found to vary greatly. Clinical cases of night blindness have been reported as cured within 24 hours by single oral administration of a large quantity of vitamin $\mathrm{A}$ (Aykroyd, 1930; Lewis and Haig, 1940) and by intramuscular injections of vitamin A concentrates (Edmund and Clemmesen, 1936). Wald, Jeghers, and Arminio (1938), and Wald and Steven (1939) reported recovery to normal visual thresholds within minutes after ingestion of mcderate concentrations of vitamin A or of carotene. Hecht and Mandelbaum (1939) obtained only slight effects with two subjects whose thresholds had risen 1 and $2 \log$ units above normal, when oral doses of vitamin A concentrates containing 100,000 units were given. McDonald and Adler (1939) were unable to lower the visual threshold with single large doses, nor were Steffens, Bair, and Sheard (1939). Hecht and Mandelbaum (1940), who administered single doses of oleum percomorphum or of a concentrate containing a high value of vitamin $A$, obtained disappointing results. Whereas single large doses 
were followed by some drop in threshold, it was only temporary. In one case the threshold, on the day following administration, had returned to its previous high position. There was no evidence from their work that single large doses of vitamin A rendered a 'cure' of night blindness. The most significant findings in the work of Hecht and Mandelbaum (1940) are that visual recovery from an A-deficient cliet is not a matter of hours and days, but of weeks and months. The fastest recovery which they recorded was made in six weeks; the slowest was not complete after three months, despite the fact that the individual received supplementary daily doses of vitamin A as high as 100,000 units plus other vitamins. This subject, however, had recovered completely after a year.

It is seen from these experiments that special dietary supplements have little or no effect upon recovery rate. It is true, also, that individuals vary greatly in their rate of depletion as well as in their rate of recovery. The reported dramatic rapid recoveries are apparently exceptions rather than the rule. The bulk of the observations emphasize the important fact that recovery from vitamin A-deficiency, as measured by dark-adaptation, is a very slow process.

Retinal Degeneration. The relation of vitamin A to the production of various ocular disturbances is a subject about which much has been written (Wolbach and Hcwe, 1925, 1933; Smith, Yudkin, Kriss, and Zimmermann, 1931; Yudkin, Kriss, and Smith, 1931; Yudkin, 1933; Yudkin, Orten, and Smith, 1937, and many others). The eye has been found to exhibit a number of pathological conditions resulting from an A-deficient diet, both in humans and in experimental animals, e.g. xerosis conjunctivae, keratomalacia, inflammation of the lacrimal gland, and others. It is not within the province of this monograph to enter into a discussion of these clinical and pathological situations, but there is one condition which merits description as a part of this treatise and this pertains to retinal degeneration. Despite the great interest in dietary night blindness and its known relationship 
to rod disfunction, the only references to structural changes in the retina prior to the work of Johnson (1939) appear to be those of Tansley $(1933 a, 1936)$.

Tansley (1933a) showed that under conditions of mild vitamin A-deficiency, in rats, the outer segments of the rods fail to take on the characteristic dark stain with phosphotungstic acid-hematoxylin. She observed also a degenerative change taking place at the junction of the inner and outer segments. In cases with severe vitamin A-deficiency she found a disappearance of the outer segments of the rods and an 'unhealthy' appearance of the whole retina. Tansley (1936) studied the development of the retina of rats suckling on A-deficient mothers, and on an A-deficient diet after weaning. The eyes of 59 young rats were examined at ages ranging from 3 to 61 days. The retina was found to have undergone normal development during the first 12 days post partem, at the end of which time the outer segments of the rods normally appear. After this period the outer segments tended to show retarded growth, but they never failed to develop. Of 23 rats killed between 12 and 21 days, 12 showed almost normal development of the retina, although only 4 had perfectly healthy outer limbs. Visual purple which normally is present at about 12 days, was not found in these $\mathrm{A}$-deficient rats until 21 days of age and then only in amounts far below the normal. In some cases no visual purple was formed. In young rats which did not receive cod-liver oil ( 7 cases), those which were killed between 44 and 58 days of age showed typical symptoms of vitamin A-deficiency, viz., poorly staining outer segments and thinning at the junction of the outer and inner segments. Dosing with cod-liver oil fer 3 days was sufficient to restore the staining reactions, but even 6 days' treatment was insufficient to repair the damage to the structure of the outer segments. Tansley's results show that the first effects of vitamin A-deficiency on young rats is either a delay or complete failure of visual purple formation, and that this is followed by structural damage to the rods. 
Johnson (1939) published a rather detailed histological description of the retina of rats suffering from mild and extreme conditions of vitamin A-deficiency. Her studies were conducted upon rats grouped into three series as follows: 1) animals on a vitamin A-free diet from the time of weaning; 2 ) animals on vitamin A-deficient diet from 8 days after birth (suckled by vitamin A-deficient mothers until weaning and then continued on an A-deficient diet); 3) animals on a vitamin A-deficient diet during suckling, and then weaned on an adequate diet. In all three series, control litters fed on adequate diets were studied along with those receiving deficient diets. These experiments were designed to test the effects of A-deficient diets on the retina when the deficiency is induced at a critical period of development (Series 2 and 3), and upon the fully differentiated retina (Series 1).

Her results showed that the retinae of rats kept upon a vitamin A-deficient diet during suckling are not markedly different from those which were not placed on a deficient diet until after weaning. In this respect her results are in agreement with those of Tansley (op. cit.) in showing that moderate vitamin A-deficiency during suckling retards development, but produces no marked structural defects, especially up to the time when the outer segments develop.

Miss Johnson's observations were made primarily upon the retinae of four groups as follows: 1) those exhibiting moderate vitamin A-deficiency, 2) those suffering from severe deficiency, 3) those fed adequately for 3 to 10 days following symptoms of severe A-deficiency, and 4) those kept on a vitamin A-deficient diet during suckling and then weaned on an adequate diet.

The retinae of all the animals with symptoms of mild vitamin deficiency showed essentially the same condition. In the peripheral portion, the rod outer segments stained very lightly as compared with the normal. In the fundus, there was definite evidence of degeneration changes. Here the retina tended to detach more readily from the pigment 
epithelial layer than in normal eyes. Not only did the rods stain abnormally, but many had lost their outer segments (Figure 107, A, cf. Figure 104). The degenerative outer segments appeared finely granular, staining very faintly or not at all. Other variations from the normal were found in this (fundus) region. The rod inner segments were swollen and presented a glassy, hyaline appearance. The external limiting membrane was found to become wavy and irregular, and in the central portion where visual cell degeneration was most marked (Figure 107, B) it had disappeared completely. The nuclei of the outer nuclear layer exhibited an edematous appearance and were pushed apart, so that their characteristic orderly arrangement was lost. The other layers of the retina showed no abnormality.

In group 2 (vide supra) eight of the twelve animals which showed symptoms of extreme vitamin A-deficiency presented similar histological features of the retina. The peripheral portion of some retinas showed more degenerative changes than those in group 1 . The outer segments were found to be very friable, most of them being broken at the junction of the inner and outer segments. In the fundus of all these retinae and in the periphery of two, the rods had disintegrated completely, leaving only pink-staining fragments of what appeared to be the remains of the inner segments (Figure 108). The external limiting membrane became lost to view and the outer nuclear layer exhibited definite signs of degenerative changes. The outermost rows of nuclei appeared to have undergone karyorrhexis. Instead of the usual 6 to 9 rows of nuclei, there were only 3 to 5 rows. The nuclei appeared pyknotic and the coarse, heavily stained chromatin granules became clumped so as to give to the nuclei a definite crenated appearance.

In some of the retinae, the degenerative changes towards the fundus were more extreme. In these cases the visual cells, the external limiting membrane, most of the outer nuclear layer, and part of the outer molecular layer had disappeared completely (cf. regions A and B, Figure 109). 


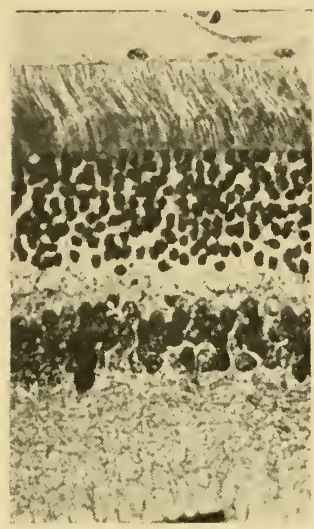

FIG. 104

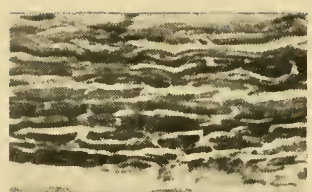

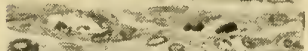

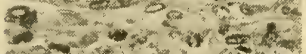

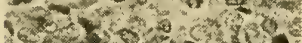

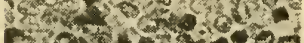

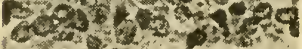

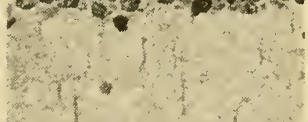
and ares. FIG. 105

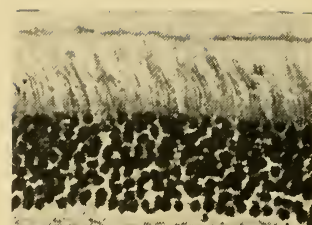
wo 020

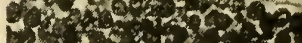

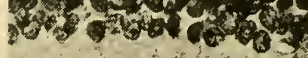

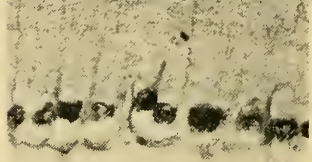

Fig. 106
A

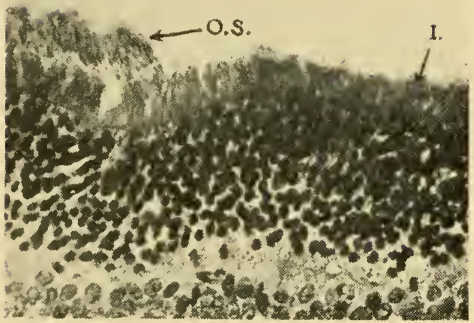

FIG. 107

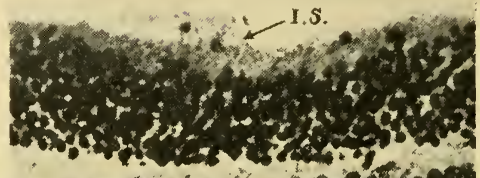

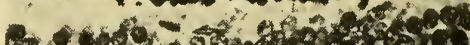

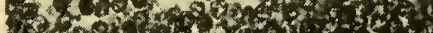

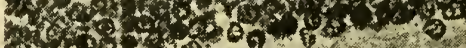

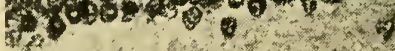
a.

FIG. 108

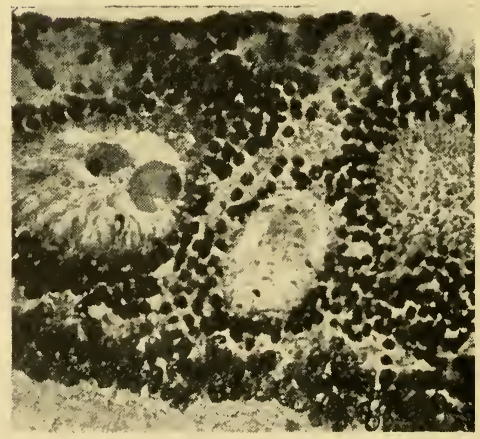

FIG. 110

FIG. 109 
Figures 104 to 110 are photomicrographs of the rat retina $(X 400)$ and are reproduced from an article by Johnson, 1939, J. Exp. Zool., vol. 81. See below for separate captions.

FIG. 104. Section through the fundus of a normal, dark-adapted retina.

FIG. 105. Section from central portion of the fundus of the retina shown in figure 109. The visual cells, the outer nuclear layer, the outer molecular layer, and most of the inner nuclear layer have completely degenerated and have been replaced by connective tissue. The epithelial pigment layer is entirely absent and the choriocapillaris is firmly joined to the retina by connective tissue (cf. figure 104).

FIG. 106. Photomicrograph of a section of the retina (fundus) from rat which had been fed adequately for 10 days following severe vitamin A-deficiency.

FIG. 107. Section through the fundus of the retina from a rat with symptoms of moderate vitamin A-deficiency. A) The few remaining rod outer segments (O.S.) are broken off at the junction with the inner segments. B) The outer segments are entirely gone, and the inner segments (I) are degenerating. The external limiting membrane is not discernible (cf. figure 104).

FIG. 108. Section through the periphery of the retina from a rat suffering from severe vitamin A-deficiency. The rod outer segments are gone, the inner segments (I.S.) are degenerating, the external limiting membrane is not disrernible, and the outer rows of visual cell nuclei are degenerating.

FIG. 109. Section through the periphery of the retina from a rat suffering from severe vitamin A-deficiency. In the region A, a few rod inner segments remain, the external limiting membrane is gone and the outer nuclear layer has begun to degenerate. In region $B$, the visual cell layer is entirely gone and the remaining visual cell nuclei are pyknotic or karyorrhectic.

FIg. 110. Section from the retina (fundus) of a rat suffering from severe vitamin A-deficiency, showing rosette formation. The deformity involves only the visual cell layer and the outer nuclear layer. 
Still further centrally, in some cases, the outer molecular layer became entirely lost, and the inner nuclear layer was reduced in width, showing definite degenerative changes. In some regions only 2 or 3 rows of bipolar cells remained. These had lost their chromatin and exhibited a pale pink stain with erythrosin (Figure 105).

It is of interest and possibly significant to point out that the epithelial pigment layer is always present and apparently normal except where the visual cells and the outer nuclear layer are absent. The loss of these two layers is always accompanied by a loss of the pigment epithelial layer (Figure 105). When this layer is not present, what remains of the retina is attached firmly to the choriocapillaris by a layer of connective tissue, which in many places is as thick as the sclera (Figure 105).

In all of the above cases, the blood vessels throughout the retina and choroid were engorged with blood, and the connective tissue of the choriocapillaris was increased in amount.

Five retinas in this group (2) showed the presence of rosettes similar to those produced experimentally by Tansley (1933b) and by Glücksmann and Tansley (1936). These retinal rosettes are produced by infoldings of the outer nuclear layers to form irregular-shaped pockets, which are lined with visual cells (Figure 110). The condition is frequently accompanied by a corresponding disarrangement of the remaining layers of the retina. The rods lining the rosettes were found to have undergone degeneration, so that the lumen became more or less filled with debris (Figure 110).

In group 3 the animals were fed on an adequate diet with supplements of cod-liver oil for 3 to 10 days after showing signs of vitamin A-deficiency. The retinae of rats killed 3 days after replacement on an adequate diet showed degenerative changes typical of severe vitamin A-deficiency, although there was less edema and less engorgement of blood vessels than in those killed in the acute stage of deficiency.

Retinae of animals killed 5 days after being on a recovery 
diet were not uniform histologically. In one case the retina appeared practically normal in the periphery, and the fundus exhibited what seemed to be newly formed rod outer segments. They appeared as short, slender protoplasmic processes without any sign of fracture at the junction of the inner and outer segments - a condition characteristic of degenerating rods. In two cases the periphery of the retina showed broken and abnormally stained rods and in the fundus the condition was more severe. In some parts of the fundus, the rods and most of the outer nuclear layer were lacking completely.

The retinae of two animals kept on a recovery diet for 10 days appeared quite normal in the periphery. All of the layers in the fundus were normal except the visual cell layer. Here the rods, like those of the 5-day recovery animals, appeared to have newly regenerated outer segments. (Figure 106).

The retinae of animals kept on a vitamin A-deficient diet during suckling and then weaned on an adequate diet (group 3) showed no signs of A-deficiency. The retinae were normal in every respect.

The results of Miss Johnson's studies have shown, in general, that with mild vitamin A-deficiency the retina exhibits abnormal staining and friability of the outer segments at the periphery, and more or less complete degeneration of the rod outer limbs in the fundus. These findings are in general agreement with those of Tansley $(1933 a, 1936)$ upon rats suffering from more severe A-deficiency. The retinae of rats suffering from severe A-deficiency were found by Miss Johnson to show more marked degeneration which was progressive, involving in succession the rod outer segments, the inner segments, the external limiting membrane, the outer nuclear layer, the epithelial pigment layer, the outer molecular layer, and the inner nuclear layer. No definite sign of degeneration was found in the inner molecular layer, the ganglion cell layer, and the nerve fiber layer.

Although there were some indications that degenerated 
rod outer segments may regenerate following the replacement on a recovery diet with cod-liver oil supplementation, the evidence was not conclusive, for there was no way of knowing what the condition of the retina was before the animal was placed on the recovery diet. In order to obtain critical data it became necessary to enucleate one eye when the animal was in an extreme state of vitamin A-deficiency, and the other eye after a certain length of time on a recovery diet. Such experiments have been carried out recently by Miss Johnson (1943), who showed that retinas with complete degeneration of the rod outer segments, partial degeneration of the inner segments and some damage of the visual cell nuclei, are capable of a remarkable degree of recovery, but only after ten to eighteen weeks of adequate feeding. Some of the rods in these cases (those whose nuclei have degenerated) apparently are lost. Although these 'repaired' rods were found to be thinner and less heavily stained than normal ones at the time of examination (after ten to eighteen weeks of adequate diet), it is entirely possible that they might have become completely normal after a longer period of recovery. Miss Johnson is of the opinion, from her findings, that in certain cases the rods have regenerated completely new outer segments, and perhaps, in some cases, new inner segments as well. No regeneration of visual cell nuclei was found in any of her cases. It is apparent, therefore, from her findings that the outer and inner segments of the rat visual rods are capable of regeneration following the prolonged administration of an adequate diet.

Hart and Guilbert (1937) have reported cases of permanent night blindness in cattle and sheep resulting from severe A-deficiency, even though ample amounts of vitamin A were administered for months with the view of effecting a cure. No histological examination of the eyes was made, and the permanence of the night blindness was attributed to optic nerve degeneration which was followed by stenosis of the optic canal. Guilbert, Howell, and Hart (1940), on the other hand, have been able to cure night blindness in 
horses by increasing the carotene or vitamin $\mathrm{A}$ intake after experimentally producing this condition by a vitamin A-deficient diet. They give in tabular form the amounts necessary to bring about night blindness, and the increased quantities of either carotene or vitamin A required to restore normal vision. It would be of interest to know, in the light of the findings of Johnson and of Tansley, whether the primary lesion which Hart and Guilbert obtained in sheep and cattle may not have been in the retina, with the optic nerve involved secondarily. In cases of greatly raised rod visual thresholds in humans, where the return to normal does not occur for many weeks and months, despite adequate diets and supplementary vitamin A administration (Hecht and Mandelbaum, 1939, 1940; McDonald and Adler, 1939), one wonders whether there may not have been some structural degeneration of the rods. If degenerative changes of the rod outer segments occur in rats following prolonged deficiencies, as has been shown by Johnson and by Tansley, there seems to be no reason why this same condition might not occur in man. Furthermore, in view of the fact that the rod outer segments are capable of regeneration in rats, as has been demonstrated by Johnson (op. cit.), it is not inconceivable theoretically that the same may occur in humans, and thus be a definite factor in the long period required by many persons to recover normal vision after vitamin A-depletion. At least it would seem pertinent for the ophthalmologists to consider this possibility as a problem for further investigation. 


\section{CHAPTER XII}

\section{RECAPITULATION}

In the foregoing chapters an attempt has been made to present a general account of the visual cells of vertebrates, with particular emphasis upon their development, structure, distribution, and certain aspects of their physiology.

That the retina, in general, possesses two types of visual elements, viz., rods and cones, has been known since its discovery by Max Schultze in 1866. Elaborations of his findings led to the so-called Duplicity Theory which implies that colorless (scotopic) vision at low illuminations is mediated by the rods, and that the cones are concerned primarily with vision at high illuminations and with color (photopic) vision.

Despite certain objections, the duplicity theory has stood the tests of physiologists in their quantitative measurements of the major functions of vision, viz., dark-adaptation, intensity discrimination, visual acuity, and flicker responses. The existence of structural and functional duality of the retina in the majority of forms is a fact of common knowledge, and animals possessing both types of visual cells are equipped for both diurnal and nocturnal vision. Many animals, however, are exclusively cone-bearing and are essentially diurnal in their behavior, whereas others are exclusively rod-bearing, and are typically nocturnal in their habits. Thus the mode of life of the animal appears to be correlated closely with the structure of the retina. Even from an histological examination of the retina, one can predict with some assurance, something of the habits of the animal as well as its visual ability. Many examples demonstrating the correlation between retinal structure and the mode of life of the animal are given in Chapter V. 
The existence of photomechanical changes of the rods, cones, and epithelial pigment in response to illumination of the eye has long been recognized. These changes consist of the forward migration of the epithelial pigment, contraction of the cones, elongation of the rods in the light, and the inverse changes in darkness. Whereas these structural changes are extensive in the eyes of fishes, anuran amphibians, and birds, they are slight in reptiles and their occurrence has never been demonstrated with conviction in the eyes of mammals and man. Although much has been written concerning the supposed significance of these changes in relation to adaptation of the eye to light and darkness, the various explanations have never fully satisfied all the conditions, due to the lack of uniformity of the responses throughout the vertebrate groups. Chapter VI gives a more or less detailed account of the photomechanical responses including various factors which influence them such as light, temperature, etc. Different theories held to account for the adaptive significance of these responses are taken up in Chapter VII where it is pointed out that none offer an all-inclusive solution.

Although there are a number of factors which influence. visual acuity, it is apparent that one of these, and an important one, pertains to the size and numbers of visual cells per unit area in the retina. A low visual acuity signifies that the average distance between the active retinal elements is large, whereas a high visual acuity indicates that the distance is small. It is obvious, therefore, that vertebrate retinas must vary greatly in this respect. Since the number of rods and cones is fixed structurally in any given area of the retina, it is apparent that they must possess different physiological thresholds in order to mediate the great variations in visual acuity accompanying changes in illumination.

In most vertebrate animals some degree of retinal specialization occurs in the fundus for increased visual acuity. This may be very slight in some forms and involve merely a greater crowding of the elements per unit area in this region. 
In others, the crowding is so great as to cause a local thickening in the retina. This is the area centralis retinae. Further specialization for increased visual acuity constitutes the foveas which are found in some salt water fishes, diurnal lizards, birds, monkeys, the great apes, and man. These various specializations of the fundus for increased visual acuity are described in Chapter VIII. The generally held view that the fovea has been developed to allow light to fall directly upon the photoreceptors without passing through all the layers of the retina, has been challenged by Walls (1937), who has shown that this structure must be viewed as a mechanism for increasing the resolving power of the retina. This matter is taken up fully in Chapter IX, which contains also a discussion of certain evolutionary relationships of the fovea. It is pointed out that whereas foveal development and the ability for extensive movements of the eyes appear to go together, conjugate movements, binocular vision, and partial decussion of the optic tracts are not necessarily implied. A critique is offered of G. Elliott-Smith's contention that marked evolutionary advances in the development of the anthropoid brain are due to the presence of the macula.

It is recognized that both rod and cone vision is initiated by the decomposition of photochemical substances in the outer segments of the visual cells. In connection with rod function, the photopigment rhodopsin (visual purple) has been known since its discovery by Franz Boll in 1876 . Although this substance has been studied by many physiologists, in recent years it has been subjected to an exhaustive spectrophotometric analysis by Wald, who has shown that vitamin $A$ is the precursor of visual purple as well as the product of its decomposition. Visual purple is a conjugated protein, the special properties of which involve principally a colored prosthetic group. This is derived from the widely distributed class of yellow to red highly saturated lipoidal pigments known as the carotenoids. In fresh water fishes as well as in those which spawn in fresh water, Wald found 
the rod photopigment to be slightly different from rhodopsin, in possessing an additional double bond in the carotenoid chain. He has termed this substance porphyropsin, which in its final bleaching in the eye is transformed into vita$\min \mathrm{A}_{2}$.

The properties of both rhodopsin and porphyropsin and their relations to the vitamins $A$ are discussed fully in Chapter $\mathrm{X}$ wherein a description is also given of certain histological entities in the fixed retina which are believed, at least tentatively, by the author to be identified with retinene - the intermediate decomposition product between vitamin $\mathrm{A}$ and visual purple.

The dependence of both rod and cone vision upon the presence of vitamin $\mathrm{A}$ in the diet is now very generally known. Whereas night blindness has long since been recognized as due to dietary deficiencies, it has been in recent years only that this problem has undergone vigorous experimental investigation in animals and man. The data obtained from many of these experiments are presented in Chapter XI. Here also are presented recent observations which not only show that vitamin A deficiencies affect the physiological thresholds of the visual elements, but that prolonged avitaminosis A may bring about structural breakdown of the rods. Evidence is presented also to show that degenerated rods in rats are able to undergo repair following prolonged feeding on an adequate diet. The theoretical possibilities of such structural changes occurring in the human retina are considered in relation to the varying periods of time required by many persons to recover normal vision after vitamin $\mathrm{A}$ depletion. 



\section{BIBLIOGRAPHY}

ANGeluccI, A. 1878 Histologische Untersuchungen über das retinale Pigment-ephithel der Wirbelthiere. Arch. f. Anat. u. Physiol., S. 353. 1884 Una nuovä theoria sulla visione. Acad. med. d. Roma. sed 14 Juglio (Cited from Garten).

— 1894 Untersuchungen über Sehtätigkeit der Netzhaut und des Gehirns. Moleschott's Untersuchungen zur Naturlehre, Bd. 14 (Cited from Garten).

Arcoleo, E. 1890 Osservazioni sperimentali sugli elementi contrattili della retina negli animali a sangue freddo. Ann. d'Ottalmologia, Anno 19, Fasc. 3e4, p. 253.

AREy, L. B. 1915 The occurrence and the significance of photomechanical changes in the vertebrate retina - an historical survey. J. Comp. Neur., v. 15, p. 535.

$1916 a$ The movements in the visual cells and retinal pigment of the lower vertebrates.. J. Comp. Neur., v. 26, p. 121.

1916b The function of the efferent fibers in the optic nerve of fishes. J. Comp. Neur., v. 26, p. 213.

$1916 c$ Changes in the rod-visual cells of the frog due to the action of light. J. Comp. Neur., v. 26, p. 429.

— 1919 A retinal mechanism of efficient vision. J. Comp. Neur., v. 30, p. 343.

1928 Visual cells and retinal pigment. Section 25. Special Cytology - Cowdry, v. 2, p. 889. Paul B. Hoeber Inc., New York.

ARey, L. B., and G. H. Mundt. 1941 A persistent diurnal rhythm in visual cones. Anat. Rec., v. 79, Suppl. 2, p. 5.

Ayкroyd, W. R. 1930 Functional night-blindness due to vitamin deficiency. Lancet, v. 1, p. 824.

Ayres, W., and W. KÜhNe. 1878 Ueber Regeneration des Sehpurpurs beim Säugethiere. Untersuch. aus d. Physiol. Inst. d. Univ. Heidelberg, Bd. 2, Heft. 2, S. 215.

BACH, I. 1895 Die Nervenzellenstruktur der Netzhaut in normalen und pathologischen Zuständen. Von Graefe's Arch. f. Ophthal., Bd. 41, Abt. 3, S. 62.

Ballantyne, J. W. 1904 Manual of antenatal pathology and hygiene. The embryo. William Green \& Sons, Edinburgh. 
Bernard, H. M. 1903 Studies in the retina. Q. J. Micro. Sci., N. S., v. 42 , p. 23 ; v. 44 , p. 443 ; v. 46 , p. 25.

Birch-Hirschfeld, A. 1900 Beitrag zur Kenntniss der Netzhautganglienzellen unter physiologischen und pathologischen Verhältnissen. Von Graefe's Arch.f. Ophthal., Bd. 50, Abt. 1, S. 155.

1906 Der Einfluss der Helladaptation auf die Struktur der Nervenzellen der Netzhaut nach Untersuchungen an der Taube. Von Graefe's Arch.f. Ophthal., Bd. 63, S. 85.

Boll, F. 1877 Zur Anatomie und Physiologie der Retina. Arch. f. Anat. u. Physiol., S. 4.

1881 Thesen und Hypothesen zur Licht- und Farbenempfindung. Arch. f. Anat. u. Physiol., S. 1.

Booher, L. E., E. C. Callison, and E. M. Hewston. 1939 An experimental determination of the minimum vitamin A requirements of normal adults. J. Nutrition, v. 17, p. 317.

Brauer, A. 1908 Ergeb. d. deut. Tiefsee - Exped. a. d. Dampfer Valdivia, Bd. 15, S. 266 (Cited from Duke-Elder, v. 1, 1939).

Cajal, S. Ramón y 1893 La Rétine des Vertébrés. La Cellule T. 9, Fase. 1, p. 119.

— 1894 Die Retina der Wirbelthiere. Weisbaden. Verlag von J. F. Bergmann.

— 1911 Histologie du système neuveau de l'homme et des vertébrés. Paris. A. Maloine.

Cameron, J. 1905 The development of the retina in Amphibia. J. Anat. and Physiol., v. 39, p. 135, 332, 471.

- 1911 Further researches on the rods and cones of vertebrate retinae. J. Anat. and Physiol., v. 46, p. 45.

Carlson, A. J. 1904 Changes in the Nissl's substance of the ganglion and the bipolar cells of the retina of the Brandt Cormorant Phalacrocorax penicillatus during prolonged normal stimulation. $A m . J$. Anat., v. 2, p. 341.

ChACE, R. R. 1943 Structural changes in the external geniculate body of the rat following removal of the eyes. Arch. Ophthal. (In Press).

Chiarini, P. 1904 Changements morphologiques que l'on observe dans la rétine des vertébrés par l'action de la lumière et de l'obscurité. Arch. ital. de Biol., T. 42, p. 303.

1906 Changements morphologiques qui se produisent dans la rétine des vertébrés par l'action de la lumière et de l'obscurité. Deuxième partie. La rétine des reptiles, des oiseux et des mammifères. Arch. ital. de Biol., T. 45, Fasc. 3, p. 337. 
Chievitz, J. H. 1889 Untersuchungen über die Area centralis Retinae. Arch.f. Anat. u. Physiol., Suppl. Bd., S. 139.

_- 1891 Ueber das Vorkommen der Area centralis Retinae in den vier höheren Wirbelthiereclasse. Arch. f. Anat. u. Physiol., Anat. Abt., S. 311.

Crozier, W. J., and G. Pincus. 1927 On the equilibration of geotropic and phototropic excitations in the rat. J.Gen.Physiol., v. 10, p. 419.

Crozier, W. J., E. Wolf, and G. Zerrhan-Wolf. 1937 Critical illumination and flicker frequency in related fishes. J. Gen. Physiol., v. 21, p. 17.

1938-1939. The flicker response function for the turtle Pseudemys. J. Gen. Physiol., v. 22, p. 311.

1939 The flicker response contour for the isopod Asellus. J. Gen. Physiol., v. 22, p. 451.

Crozier, W. J., and E. Wolf. 1939 Temperature and critical illumination for reaction to flickering light. IV. Anax nymphs. J. Gen. Physiol., v. 22, p. 795.

- 1939 The flicker response contour for the crayfish. I. J. Gen. Physiol., v. 23, p. 1.

1939 The flicker-response contour for the crayfish. II. Retinal pigment and the theory of the asymmetry of the curve. Biol. Bull., v. 77, p. 126.

1939 The flicker response contours for genetically related fishes. II. J. Gen. Physiol., v. 22, p. 463.

1939 Temperature and critical illumination for reaction to flickering light. III. Sunfish. J. Gen. Physiol., v. 22, p. 487.

1939 The flicker response contour for the frog. J. Gen. Physiol., v. 23, p. 229 .

1939 The flicker response contour for the gecko (rod retina). J. Gen. Physiol., v. 22, p. 555.

1939 The flicker response contour for Phrynosoma (horned lizardcone retina). J. Gen. Physiol., v. 24, p. 317.

1940 The flicker response curve for fundulus. J. Gen. Physiol., v. 23, p. 677.

1940 Reaction to visual flicker in the newt Triturus. J. Gen. Physiol., v. 23, p. 667 .

1941 The simplex flicker threshold contour for the zebra finch. J. Gen. Physiol., v. 24, p. 625.

Czerny, V. 1867 Ueber Blendung der Netzhaut durch Sonnenlicht. Sitzb. d. Akad. d. Wiss. zu Wien, Math. Natur. Kl., Bd. 56, Abt. 2, S. 409. 
Detwiler, S. R. 1916 The effect of light on the retina of the tortoise and the lizard. J. Exp. Zool., v. 20, p. 165.

- 1920 The structure of the retina of Phrynosoma cornutum. J. Comp. Neur., v. 32, p. 347.

- $1923 a$ Studies on the retina. Photomechanical responses in the retina of Eremias argus. J. Exp. Zool., v. 37, p. 89.

$1923 b$ Studies on the retina. The identity of the developing visual cells in Amblystoma larvae as revealed by their responses to light. J. Comp. Neur., v. 36, p. 113.

- $1923 c$ Studies on the retina. An experimental study of the gecko retina. J. Comp. Neur., v. 36, p. 125.

1924 Studies on the retina. Observations on the rods of nocturnal mammals. J. Comp. Neur., v. 37, p. 481.

1932 Experimental observations upon the developing rat retina. J. Comp. Neur., v. 55, p. 473.

1939 Comparative studies upon the eyes of nocturnal lemuroids, monkeys and man. Anat. Rec., v. 74, p. 129.

1940 The eye of Nycticebus tardigradus. Anat. Rec., v. 76, p. 295.

- 1941 The eye of the owl monkey (Nyctipithecus). Anat. Rec., v. 80, p. 233.

Detwiler, S. R., and H. Laurens. 1921 Studies on the retina. Histogenesis of the visual cells in Amblystoma. J. Comp. Neur., v. 33, p. 493.

Detwiler, S. R., and R. Lewis. 1926 Temperature and retinal pigment migration in the eyes of the frog. J. Comp. Neur., v. 41, p. 153.

Detwiler, S. R., and R. L. Zwemer. 1937 On the nature of the so-called droplets found between the rod outer segments of vertebrate eyes. Anat. Rec., v. 67, p. 295.

DeVoss, J. C., and R. Ganson. 1915 Color blindness in cats. J. Animal Bchavior, v. 5, p. 115.

Dogiel, A. 1888 Über Verhalten der nervösen Elemente in der Retina der Ganoiden, Reptilier, Vögel und Saugetiere. Anat. Anz., Bd. 3, S. 133 .

Dreser, H. 1886 Zur Chemie der Netzhautstäbchen. Zeitsch. f. Biol., Bc. 22, S. 23.

DUke-Elder, W. S. 1939 Textbook of ophthalmology. Vol. I. The development, form, and function of the visual apparatus. C. V. Mosby Company, St. Louis.

Edmexd, C., and S. Chemmesen. 1936 On deficiency of A vitamin and risual dysaptation. $92 \mathrm{pp}$. Copenhagen. 
ElLiotт-Sмith, G. 1928 The new vision. Bowman Lecture. Trans. Ophth. Soc., United Kingdom, v. 48.

Engelmann, T. W. 1885 Über Bewegungen der Zapfen und Pigmentzellen der Netzhaut unter dem Einfluss des Lichtes und des Nervensystems. Pflüger's Arch., Bd. 35, S. 498.

Ewald, H., and W. KüHNe. 1878 Untersuchungen über den Sehpurpur.

II. Entstehung der Retinafarbe. Untersuch. physiol. Inst. Heidelberg, Bd. 1, S. 248.

Exner, S., and H. Januschke. 1906 Die Stäbchen wanderung im Auge von Abramis brama bei Lichtveränderungen. Ber. d. K. K. Akad.d. Wiss. zu Wien, Math.-Natur. Kl., Bd. 115, S. 269.

Fick, A. E. 1891 Untersuchungen über die Pigmentwanderung in der Netzhaut des Frosches. Von Graefe's Arch.f. Ophthal., Bd. 37, S. 1.

Franz, V. 1913 Sehorgan. Oppel's Lehrbuch d. verg. mikros. Anat. d. Wirbeltiere. G. Fisher, Jena.

1934 Vergleichende Anatomie des Wirbeltierauges. Handb. d. verg. Anat. d. Wirbeltiere, Bd. 2, S. 1161.

Fridericia, L. S., and E. Hols. 1925 Experimental contribution to the study of the relation between night blindness and malnutrition. Influence of deficiency of fat-soluble A-vitamin in the diet on the visual purple in the eyes of rats. Am. J. Physiol., v. 73, p. 63.

Fujita, H. 1911 Pigmentbewegung und Zapfenkontraction im Dunkelauge des Frosches bei Einwirkung verschiedener Reize. Arch.f. verg. Ophthal., Bd. 2, S. 164.

GAD, J. 1894 Der Energieumsatz in der Retina. Arch,f. Anat. u. Physiol., Physiol. Abt. Jahrg., S. 491.

Garten, S. 1907 Die Veränderungen der Netzhaut durch Licht. Von Graefe-Saemisch Handb. d. Augenheilk., Teil 1, Bd. 3, Kap. 12, Anhang. 2. Aufl., S. 250.

GlüCKsmann, A., and K. TANSLEy. 1936 Some effects of gamma radiation on the developing rat retina. Brit.J. Ophthal., v. 20, p. 497.

GreefF, R. 1900 Die mikroskopische Anatomie des Sehnerven und der Netzhaut. Von Graefe-Saemisch Handb. d. Augenheilk., Bd. 1, Abt. 2, Kap. 5, S. 1. Leipzig. Engelmann 1900.

Gridgeman, N. T., and H. Wilkinson. 1938 Night blindness and vitamin A deficiency: The use of the biophotometer. Lancet, v. 1, p. 905.

Guilbert, H. R., C. E. Howell, and G. H. Hart. 1940 Minimum vitamin $\mathrm{A}$ and carotene requirements of mammalian species. J. Nutrition, v. 19, p. 91.

Haig, C., S. Несht, and A. J. PAтek. 1938 Vitamin A and rod-cone adaptation in cirrhosis of the liver. Science, v. 87, p. 534. 
Hamburger, D. J., 1889 Dorsnijding van der nervus opticus bij Kikvorschen, in Verband met de Beweging van Pigment en Kegels in het Netvlies, onder den Invloed van Licht und Duister. Onderzoekingen d. Utrechtsche Hoogeschal, Reeks 3, v. 11, p. 58.

Harris, W. 1904 Binocular vision and stereoscopic vision in man and other vertebrates, with its relation to the decussation of the optic nerves, the ocular movements and the pupil light reflex. Brain, v. 27, p. 107.

Hart, G. H., and H. R. Guilbert. 1937 Symptomology of vitamin A deficiency in domestic animals. J. Am. Vet. Med. Assoc., v. 91, p. 193.

Hartridge, H. 1919 The shape of the pupil in various animals. J. Physiol., v. 53, p. 6.

Hecht, S. 1920 The dark adaptation of the human eye. J. Gen. Physiol., v. 2, p. 499 .

- 1927 The kinetics of dark adaptation. J.Gen. Physiol., v. 10, p. 781.

1928 Visual acuity and illumination. Arch.Ophthal., v. 57, p. 564.

- 1936 The regeneration of visual purple in solution. Science, v. 84, p. 331.

—_ 1937 Rods, cones and the chemical basis of vision. Physiol. Rev., v. 17, p. 239 .

— 1938a The nature of the visual process. Bull. New York Acad. Med., v. 14, p. 21.

- $1938 b$ Rod-cone dark adaptation and vitamin A. Science, v. 88, No. 2279, p. 219.

Hecht, S., A. M. Chase, and S. Shlaer. 1937 The diffusion coefficient and molecular size of visual purple. Science, v. 85, p. 567.

Нecht, S., and J. Mandelbaum. 1939 The relation between vitamin A and dark adaptation. J. Am. Med. Assoc., v. 112, p. 1910.

1940 Dark adaptation and experimental vitamin A deficiency. Am. J. Physiol., v. 130, p. 651.

Hecht, S., and S. Shlaer. 1938 An adaptometer for measuring human dark adaptation. J. Optical Soc. Amer., v. 28, p. 269.

Неснт, S., and R. Williams. 1922 The visibility of monochromatic radiation and the absorption spectrum of visual purple. J. Gen. Physiol., v. 5, p. 1.

Herzog, H. 1905 Experimentelle Untersuchungen zur Physiologie der Bewegungsvorgänge in der Netzhaut. Arch. f. Anat. u. Physiol. Phys. Abt., S. 413. 
Hess, C. 1909 Untersuchungen zug vergleichenden Physiologie und Morphologie des Accommodationsvorgänges. Arch. f. Augenheilk., Bd. 62 , S. 345 .

1910 Untersuchungen über den Lichtsinn bei Reptilien und Amphibien. Pflüger's Arch., Bd. 132, S. 255.

1913 Gesichtssinn. Winterstein's Handb. f. verg. Physiol., Bd. 4, S. 555. G. Fischer.

IsaAcs, B. L., V. T. Jung, and A. C. Ivy. 1938 Vitamin A deficiency and dark adaptation. J. Am. Med. Assoc., v. 111, p. 777.

JeAns, P. C., and Z. Zentmire. 1934 A clinical method for determining moderate degrees of vitamin A deficiency. J.Am. Med.Assoc., v. 102, p. 892.

JEGHERs, H. 1937 The degree and prevalence of vitamin A deficiency in adults. J. Am. Med. Assoc., v. 109, p. 756.

Johnson, G. L. 1901 Contributions to the comparative anatomy of the mammalian eye, chiefly based on ophthalmoscopic examination. Phil. Trans. Roy. Soc. B., v. 194, p. 1.

Johnson, M. L. 1935 Visual cells of the amphibian retina in the absence of the epithelial pigment layer. Anat. Rec., v. 63, p. 53.

1939 The effects of vitamin A deficiency upon the retina of the rat. J. Exp. Zool., v. 81, p. 67.

1943 Degeneration and repair of the rat retina in avitaminosis A. Arch. Ophthal. (In Press).

Johnson, M. L., and S. R. Detwiler. 1942 On the relation of certain droplets in the vertebrate retina to the visual cycle. J. Exp. Zool., v. 89, p. 233.

Kahmann, H. 1936 Über das foveale Sehen der Wirbeltiere. (1. Über die Fovea centrales und die Fovea lateralis bei einigen Wirbeltieren.) Von Graefe's Arch. f. Ophthal., Bd. 135, S. 265.

Karsten, H. 1923 Das auge von Periophthalmus Koelreuteri. Jena. Zeit.f. Naturw., Bd. 59, S. 115.

Katz, D., and G. RÉvész. 1914 Ein-Beitrag zur Kenntniss des Lichtsinns der Nachtvögel. Zeit. f. Psychol. u. Physiol. d. Sinnesorg. Abt. 2. Zeit. f. Sinnesphysiol., Bd. 48, S. 165.

KeELER, C. 1927 Absence héréditaire des batônnets chez la souris, mus musculus. Étude histologique. Mem. de la Soc. Zool. de France, T. 28, p. 48 .

KerR, J. G. 1919 Textbook of embryology. Vol, 2. Macmillan \& Company, London. 
Koenig, A. 1894 Ueber den menschlichen Sehpurpur und seine Bedeutung für das Sehen. Sitzungber. Akad. Wiss., Berlin (Cited from Hecht and Williams, 1922).

Kolmer, W. 1909 Ueber einensekretartigen Bestandteil der StäbschenZapfen schicht der Wirbeltiere. Arch.f. d. ges. Physiol., Bd. 129, S. 34 .

1925 Bemerkungen über Adaptionsvorgänge in den Sehelementen. Arch. f. Ophthal., Bd. 115, S. 310.

1936 Die Netzhaut (Retina). v. Möllendorff's Handb. d. mikroskopischen Anat. des Menschen., Bd. 3, S. 295.

Köttgen, E., and G. AbelsdorfF. 1896 Absorption und Zersetzung des Sehpurpurs bei den Wirbeltieren. Z. Psychol. u. Physiol. Sinnesorgane. Bd. 12, S. 161.

Krause, W. 1894 Die Retina. 5. Die Netzhaut des Vögel. Internat. Monatsch.f. Anat. u. Physiol., Bd. 2, S. 1.

KüHne, W. 1877 Ueber das Vorkommen des Sehpurpurs. Untersuch. aus. d. Physiol. Inst. d. Univ. Heidelberg. Centralbl. Med. Wiss., Bd. 15, S. 257.

- 1878 Fortgesetzte Untersuchungen über die Retina und die Pigmente des Auges. Unl.rsuch. aus. d. Physiol., Inst. d. Univ. Heidelberg. Bd. 2, Heft 1, S. 89.

—— 1879 Chemische Vorgänge in der Netzhaut. Hermann's Handb. des Physiologie, Leipzig. Bd. 3, Teil 1, S. 235.

Kühne, W., and H. Sewall. 1880 Zur Physiologie des Sehepithels, inbesondere der Fische. Untersung. aus. d. Physiol. Inst. Univ. Heidelberg. Bd. 3, S. 221.

Laurens, H., and S. R. Detwiler. 1921 The structure of the retina of Alligator mississippiensis and its photomechanical changes. J.Exp. Zool., v. 32, p. 207.

LeboucQ, G. 1909 Contribution a l'étude de l'histogenese de la rétine chez les mammifères. Arch. d. anat. micros., Paris, T. 16, p. 556.

Lederer, R. 1908 Veränderungen an den Stäbschen der Froschnetzhaut unter Einwirkung von Licht und Dunkelheit. Centralbl. f. Physiol., Bd. 22, S. 762.

Leplat, G. 1913 Les plastosomes des cellules visuelles et leur rôle dans la différentiation des cônes et des bâtonnets. Anat. Anz., Bd. 45, S. 215 .

Levi, G. 1901 Osservazioni sullo sviluppo dei coni e bastonici della retina degli Urodeli. Lo Sperimentale Archivio di biologia normale e patologica. Anno $54^{\circ}$, Fasc. 6, p. 521. 
Levi, G. 1914 Ulteriori studi sullo sviluppo delle cellule visive negli Anfibi. Anat. Anz., Bd. 47, Nr. 6-7, S. 192.

Lewis, J. M., and C. HaIG. 1940 Vitamin A status of children as determined by dark adaptation. J. Pediatrics, v. 16, p. 285.

Magitot, A. 1910 Étude sur le développement de la rétine humaine. Ann. d'oculistique. T. 143, p. 241.

Mans, G. 1895 Histological changes induced in sympathetic, motor and sensory nerve cells by functional activity. J. Anat. and Physiol., v. 29, p. 100 .

Mann, I. 1928 The development of the human eye. Cambridge Univ. Press.

McDonald, R., and F. H. Adler. 1939 Effect of anoxemia on the dark adaptation of the normal and of the vitamin A-deficient subject. Arch. Ophthal., v. 22, p. 980.

Menner, E. 1929 Untersuchungen über die Retina mit besonderer Berücksichtigung der äussern Körnerschicht. Ein Beitrag zur Duplizitätstheorie. Zeit.f. verg. Physiol., Bd. 8, S. 761.

__ 1930 Zapfen in der Retina der Maus. Zeit.f. Zellforsch. u. mikros. Anat., Bd. 11, Heft 1, S. 53.

Morano, F. 1872 Die Pigmentschicht der Netzhaut. Arch. f. mikros. Anat., Bd. 8, S. 81.

MÜLLER, H. 1856 Anatomisch-physiologische Untersuchungen über die Retina bei Menschen und Wirbeltiere. Zeitsch.f. Wiss. Zool., Bd. 8, Heft 1, S. 1.

Nahmacher, W. 1893 Über den Einfluss reflectonische und centrale Opticusreizung auf die Stellung der Zapfen in der Froschnetzhaut. Arch.f. ges. Physiol., Bd. 35, Heft 9 u 10, S. 375.

Osawa, G. 1898 Beiträge zur Lehre von den Sinnesorganen der Hatteria punctata. Arch.f. mikros. Anat., Bd. 52, S. 268.

Palmer, C. E., and H. Blumberg. 1937 The use of a dark adaptation technic (Biophotometer) in the measurement of vitamin A deficiency in children. Pub. Health Rep., v. 52, p: 1403.

Parsons, J. H. 1915 An introduction to the study of colored vision. Putnam's Sons. New York.

Pergens, E. 1896 Action de la lumière sur la rétine. Travaux de l'institut Solvoy. T. 1 (Cited from Garten).

1897 Action de la lumière colorées sur la rétine. Ann. de la Soc. Roy. des Sci. med. et Nat. de Bruxelles, T. 6 (Cited from Garten). 
Pergens, E. 1899 Úber Vorgänge in der Netzhaut bei farbiger Beleuchtung gleicher Intensität. Zeit. f. Augenheilk., Bd. 2, S. 125.

Polyak, S. L. 1941 The retina. University of Chicago Press, Chicago.

Pütter, A. 1912 Organalogie des Auges. Wm. Englemann. Leipzig.

Rochon-Duvigneaud, A. 1917 Les fonctions des cônes et des bâtonnets. Indications fournies par la physiologie comparée. Ann. d'oculistique. T. 154 , p. 633.

1919 Quelques données sur la fovea des oiseaux. Ann. d'oculistique. T. 156, p. 717.

1920 La situation des foveae simples et doubles dans la rétine des oiseaux et le problème de leurs relations fonctionelles. Ann. d'oculistique. T. 157, p. 673.

- 1923 Topographie et fonctions des foveae centrales et des foveae latérales chez les oiseaux pourvus de deux foveae rétiniennes; lignes de vision binoculaire et de vision indépendante. Ann. d'oculistique. T. 160, p. 769 .

Salzmann, M. 1912 Anatomie u. Histologie des menschlichens Augapfels im normal zustande, seine Entwicklung und sein Altern. Leipzig.

Schaper, A. Zur Histologie der menschlichen Retina. Arch.f. mikros. Anat., Bd. 41, S. 147.

Schultze, M. 1866 Zur Anatomie und Physiologie der Retina. Arch. f. mikros. Anat., Bd. 2, S. 175.

SchÜPBACH, P. 1906 Beiträge zur Anatomie und Physiologie der Ganglienzellen in Zentralnervensystem der Taube. Zeit. f. Biol., Bd. 47, S. 439 .

Seefelder, R. 1910 Beiträge zur Histogenese und Histologie der Netzhaut, des Pigmentepithels und des Sehnerven. Arch.f. Ophthal., Bd. 73, S. 419.

Sgrosso. 1905 Sul una diversità di tinzione che osservasi nella retine di rane tenute alla oscurità ed alla luce a colorate col triacido di Ehrlich. Rend. riass. de 1\%. congr. dell' assoc. ottalm. ital. Napoli (Cited from Garten).

Singer, J., and E. Münzer. 1889 Kenntniss der Sehnervenkreuzung. Denkschrift der Kais. Akad. d. Wissenschaften. Math.-Natur. Wiss. Classe, Bd. 55, S. 163.

Slonaker, J. R. 1897 A comparative study of the area of acute vision in vertebrates. J. Morph., v. 13, p. 445.

Smith, A. H., A. M. Yudkin, M. Kriss, and H. M. Zimmermann. 1931 Vitamin A content of retinal and choroidal tissue. J. Biol. Chem., v. 82, p. 92 . 
Steffens, L. F., H. L. Bair, and C. Sheard. 1939 Photometric measurements on visual adaptation in normal adults on diets deficient in vitamin A. Proc. Staff Meet. of Mayo Clinic, v. 14, p. 698.

Steininger, G., L. J. Roberts, and S. Brenner. 1939 Vitamin A in the blood of normal adults. The effect of a depletion diet on blood values and biophotometer readings. J. Am. Med. Assoc., v. 113, p. 2381.

Stort, A. G. H. van Genderen. 1886 Über Form und Ortsveränderungen der Elemente in der Sehzellenschicht nach Beleuchtung. Bericht über d.18 Versamm. Ophthal. Gesell. zu Heidelberg. S. 43.

1887 Über Form und Ortsveranderungen der Netzhautelemente unter Einfluss von Licht und Dunkel. Von Graefe's Arch.f. Ophthal., Bd. 33, Abt. 3, S. 229.

TANSLEY, K. 1931 The regeneration of visual purple: its relation to dark adaptation and night blindness. $J$. Physiol., v. 71, p. 442.

- 1933a Factors affecting the development and regeneration of visual purple in the mammalian retina. Proc. Roy. Soc., v. 114, p. 79.

$1933 b$ The formation of rosettes in the rat retina. Brit. J. Ophthal., v. 17, p. 321 .

- 1936 The effect of vitamin A deficiency on the development of the retina and on the first appearance of visual purple. Biochem. J., v. 30, p. 839.

TARTUFeri, F. 1887 Sulle cisti transparenti dell' orlo cigliare delle palpebre. Arch. per. le sc. med. Torino, v. XI, p. 17 (also in Int. Monatsch. f. Anat. u. Physiol., Leipzig, 1887, Bd. 4, S. 177).

Valentin, G. 1879 Ein Beitrag zur Kenntnis der Brechungsverhältnisse der Thiergewebe. Arch.f.d. ges. Physiol., Bd. 19, S. 78.

1879 Fortgesetzte Untersuchungen über die Brechungsverhältnisse der Thiergewebe. Arch.f. d. ges. Physiol., Bd. 20, S. 283.

Verrier, M. L. 1935 Recherches sur l'histophysiologie de la rétine des vertébrés et les problèms qu'elle soulève. Bull. Biol. de France et de Belgique. Suppl. 20, p. 1.

WaELCHI, G. 1883 Zur Topographie der gefärbten Kugeln der Vogelnetzhaut. Von Graefe's Arch. f. Ophthal., Bd. 29, S. 205.

WALD, G. $1935 a$ Vitamin A in eye tissues. J.Gen. Physiol., v. 18, p. 905.

$1935 b$ Carotenoids and the visual cycle. J. Gen. Physiol., v. 19, p. 351.

1936 Pigments of the retina. I. The bull frog. J. Gen. Physiol., v. 19, p. 781 . 
WaLd, G. 1936-1937 Pigments of the retina. II. Sea robin, sea bass, and scup. J. Gen. Physiol., v. 20, p. 45.

$1937 a$ Visual purple system in fresh water fishes. Nature, v. 139, p. 1017.

_ $1937 b$ Photo-labile pigments of the chicken retina. Nature, v. 140, p. 545 .

1938 On rhodopsin in solution. J. Gen. Physiol., v. 21, p. 795.

— 1939a The porphyropsin visual system. J. Gen. Physiol., v. 22, p. 775 .

$1939 b$ Vitamin A and vision. Collecting Net, v. 14, p. 1.

- $1942 a$ The visual system and vitamin A of the sea lamprey. J.Gen. Physiol., v. 25, p. 331.

$1942 b$ Visual systems and vitamins A. Biological Symposia, v. 7, p. 43.

WALD, G., H. JeGhers, and J. ARMinio. 1938 An experiment in human dietary night-blindness. Am. J. Physiol., v. 123, p. 732.

Wald, G., and D. Steven. 1939 An experiment in human vitamin A deficiency. Proc. Nat. Acad., v. 25, p. 344.

WALLS, G. 1928 The photo-mechanical changes in the retina of mammals. Science, v. 67, p. 655.

1932 Visual purple in snakes. Science, v. 75, p. 467.

- $1934 a$ The reptilian retina. Am. J. Ophthal., v. 17, p. 892.

- $1934 b$ Human rods and cones. Arch. Ophthal., v. 12, p. 914.

- 1937 Significance of the foveal depression. Arch. Ophthal., v. 18, p. 912.

- $1939 a$ The significance of 'Kolmer's droplets' of the vertebrate retina. Anat. Rec., v. 73, p. 373.

— $1939 b$ Origin of the vertebrate eye. Arch. Ophthal., v. 22, p. 452.

1940 Postscript on image expansion of the foveal clivus. Arch. Ophthal., v. 23, p. 831.

— $1942 a$ The vertebrate eye and its adaptive radiation. Cranbrook Inst. Sci., Bloomfield Hills, Michigan.

$1942 b$ The visual cells and their history. Biological Symposia, v. 7, p. 203.

Weigert, F. 1921 Ein photochemisches Modell der Retina. Arch. ges. Physiol., Bd. 190, S. 177. 
WeLCH, J. H., and C. M. Osborn. 1937 Diurnal changes in the retina of the catfish, Ameiurus nebulosus. J. Comp. Neur., v. 66, p. 349.

Wolbach, S. B., and P. R. Howe. 1925 Tissue changes following deprivation of fat-soluble A vitamin. J. Exp. Med., v. 42, p. 753.

1933 Epithelial repair in recovery from vitamin A deficiency. An experimental study. J. Exp. Med., v. 37, p. 511.

Wolff, E. 1940 The anatomy of the eye and the orbit. II Edition. The - Blakiston Company, Philadelphia.

Woop, C. 1917 The fundus oculi of birds. The Lakeside Press, Chicago, Illinois.

Woollard, H. H. 1925 The anatomy of Tarsius spectrum. Proc. Zool. Soc., London, p. 107.

1926 Notes on the retina and lateral geniculate body in Tupaia, Nycticebus and Hapale. Brain, v. 49, p. 77.

1927 Differentiation of the retina in the primates. Proc. Zool. Soc., London, p. 5.

Wunder, W. 1926 Physiologische und vergleichend-anatomische Untersuchungen an der Knochenfischnetzhaut. Zeit.f. Wiss. Biol., Abt. C. Physiologie, Bd. 3, S. 1.

YUDKIN, A. M. 1933 Ocular disturbances produced in experimental animals by dietary changes. Clinical implications. J. Am. Med. Assoc., v. 101, p. 921.

YudKin, A. M., M. Kriss, and A. H. SмITH. 1931 Vitamin A potency of retinal tissue. Am. J. Physiol., v. 97, p. 611.

Yudkin, A. M., A. N. Orten, and A. H. Simth. 1937 Production and cure of ocular disturbances in adult albino rats by adjustment of vitamins. Clinical implications. Am. J. Ophthal., v. 20, p. 1115. 



\section{AUTHOR INDEX}

Abelsdorf, G., 125

Adler, F. H., 149, 159

Angelucci, A., 75, 76, 78, 79

Arcoleo, E., 75, 78, 79

Arey, L. B., 42, 44, 48, 52, 74, 75, 77. $78,79,80,81,82,83,86,88,92$, 94,139

Arminio, J., 143, 144, 146, 148, 149

Aykroyd, W. R., 149

Ayres, W., 86, 120, 131

Bach, I., 84

Bair, H. I., 148, 149

Ballantyne, J. W., 11

Bernard, H. M., 54

Birch-Hirschfeld, A., 84, 85

Blumberg, H., 144

Boll, F., 74, 75, 77, 86, 120, 122, 162

Booher, L. E., 148

Brauer, 94

Brenner, S., 149

Cajal, S. R. y, 25, 54

Callison, E. C., 148

Cameron, J., 54, 56, 57

Carlson, A. J., 85

Chace, R., 115

Chase, A. M., 133

Chiarini, P., 76, 79, 84, 87

Chievitz, J. H., 49, 51, 98, 110

Clemmensen, S., 144, 149

Crozier, W. J., 37, 38, 59, 61

Czerny, V., 74, 78

Detwiler, S. R., 6, 9, 43, 44, 54, 55, 58, $61,65,74,75,76,77,78,79,80,85$, $103,131,132,133,135,136,139$

DeVoss, J. C., 65

Dogiel, A., 25

Dresser, H., 87

Duke-Elder, W. S., 3, 5, 8, 11, 13, 25, $29,40,41,48,52,109$

Edmund, C., 144, 149

Elliott-Smith, G., 66, 108, 111, 112, $113,115,162$
Englemann, T. W., 77, 79, 81

Ewald, H., 120

Exner, S., 75, 87, 88, 90

Fick, A. E., 81

Franz, V., 24, 25, 45

Fridericia, L. S., 124, 143

Fujita, H., 81

Gad, J., 86

Ganson, R., 65

Garten, S., 64, 74, 75, 76, 77, 78, 79, $84,88,90,121$

Glücksmann, A., 156

Greeff, R., 39, 62

Gridgeman, N. T., 144

Guilbert, H. R., 158, 159

Haig, C., 146, 149

Hamburger, D. J., 81

Harris, W., 112, 113

Hart, G. H., 158, 159

Hartridge, H., 14

Hecht, S., 38, 93, 94, 122, 123, 133, $143,144,145,146,148,149,150$, 159

Herzog, H., 80, 87, 139

Hess, C., 8, 63, 64, 76

Hewston, E. M., 148

Holm, E., 124, 143

Howe, P. R., 150

Howell, C. E., 158

Isaacs, B. L., 144

Ivy, A. C., 144

Januschke, H., 75, 87, 88, 90

Jeans, P. C., 144

Jeghers, H., 143, 144, 146, 148, 149

Johnson, M. L., 132, 133, 135, 151, $152,157,158,159$

Jung, V. T., 144

Kahmann, H., 113, 114

Karsten, H., 94

Katz, D., 64 
Keeler, C., 132

Kerr, J. G., 54

Koenig, A., 93, 94, 120

Kolmer, W., 46, 87, 130, 132, 133, 136

Köttgen, E., 125

Krause, A. C., 120

Krause, W., 63, 76

Kriss, M., 150

Kühne, W., 74, 75, 77, 86, 87, 120 , $121,122,125,131$

Laurens, H., 54, 61, 76, 79

Leboucq, G., 53,

Lederer, R., 79

Leplat, G., 57

Levi, G., 42, 54, 55

Lewis, J. M., 149

Lewis, R., 75, 80, 139

Magitot, A., 53, 54

Mandelbaum, J., 143, 144, 145, 146 , $148,149,150,159$

Mann, G., 84

Mann, I., 48, 51, 53, 54

McDonald, R., 149, 159

Menner, E., 19, 64, 105

Morano, F., 74

Müller, H., 74

Mundt, G. H., 83

Münzer, E., 113

Nahmacher, W., 81

Orten, A. N., 150

Osawa, G., 47

Osborn, C. M., 83

Palmer, C. E., 144

Parsons, J. H., 54

Patek, A. J., 146

Pergens, E., 75, 84

Pincus, G., 59

Polyak, S. L., 30, 31, 33, 35, 36, 40, 105

Pütter, A., 25, 45

Révész, G., 64

Roberts, L. J., 149
Rochon-Duvigneaud, A., 15, 63, 91, 103

Salzmann, M., 40

Schultze, M., 37, 38, 160

Schüpbach, P., 85

Seefelder, R., 53, 54

Sewall, H., 125

Sgrosso, 84, 85

Sheard, C., 148, 149

Shlaer, S., 133, 144

Singer, J., 113

Slonacker, J. R., 99, 102

Smith, A. H., 150

Steffens, L. F., 148, 149

Steininger, G., 149

Steven, D., 148, 149

Stort, van Genderen, A. G. H., 75, $76,77,78,79$

Tansley, K., 124, 132, 133, 143, 151, $152,156,157,159$

Tartuferi, F., 25

Valentin, G., 119

Verrier, M. L., 38

Waelchli, G., 43

Wald, G., 43, 122, 123, 124, 125, 126 , $127,128,133,135,136,142,143$, $144,146,148,149,162$

Walls, G., 1, 38, 44, 61, 62, 72, 73, 91, $104,116,117,118,119,132,162$

Weigert, F., 123

Welch, J. H., 83

Wilkinson, H., 144

Williams, R., 123

Wolbach, S. B., 150

Wolf, E., 37, 38

Wolff, E., 40, 46, 109, 110

Wood, C., 24, 98, 99, 101, 102, 109

Woollard, H. H., 66, 70, 71, 108, 111, 116

Wunder, W., 17,42

Yudkin, A. M., 150

Zentmire, Z., 144

Zimmermann, H. M., 150

Zwemer, R. L., 133 


\section{SUBJECT INDEX}

Albinism, 86

Anterior chamber, 67, 70

Area centralis retinae, 66, 71, 95, 104, $108,110,111,117,118$

Bulbus oculi, 2

Carotenoids, 122, 124, 126, 130

Cells, amacrine, 24-28, 31, 32, 35, 36, 50,51

association, 24

bipolar, 24-28, 30-33, 35, 36, 50, 51

brush, 31-33

centrifugal, $31,35,36$

centripetal, $32,35,36$

diffuse, $32,33,35$

flat, 31-33

midget, 31-35

mop, 31-34

ganglion, 24-28, 30-36, 50, 84

diffuse, $31,33,34$

midget, 31,34

shrub, 31

horizontal association, 24, 25, 31, $35,36,50,51$

"myoepithelial," 9

spider, 28

supporting, 25-28

visual (see cones and rods)

Choroid, 6

lamina vitrea of, 16

specialization of, 6,7

Ciliary body, 7

ciliary processes of, 7,8

muscles of, 8-10

relative size of, 8,9

variations of, 8,9

Ciliary muscle, $8,10,67,70$

Clivus, 110, 118

Cones, classification of, 45-47

conducting systems, 30-36

connections (endings) of, 25, 45

differentiation of, 50-58

double, 27, 28, 42, 61

function of, 37-39

in relation to visual acuity, 93-103 measurements of, 20, 22, 23, 39, 40

movements of (see photomechanical responses)

nuclei of, $39,84,85$

number of, 41

of foveal region, 22, 23, 39, 40, 103

origin of, 49

photomechanical responses of, 47

photopigment of (see iodopsin)

size of, 20, 94, 95, 103

staining reaction of, 46

structure of, 39,42

thresholds of, 37, 94

twin, 26, 42

Cornea, measurements of, 70

of nocturnal animals, $3,5,6,67,70$

refractive power of, 5

shape of, 5

size of, in various vertebrates, 5,6 , $67-70$

Corona ciliaris (pars plicata), 8

Dark adaptation, 121, 144-150

Diplosome, of cones and rods, 53, 54

Diurnal rhythms, 83

Double retina of Parinaud, 37

"Droplets" of Kolmer, 129, 130

in relation to dark- and light-adaptation, 131, 135-142

in relation to pigment epithelial layer, 130, 132

in relation to retinene, $133-142$

in relation to rhodopsin, 87,130 , 132-136, 140, 141

in relation to vitamin A, 132-142

nature of, 133

occurrence of, 130-132

origin of, 130, 132

reaction to illumination changes, 135-142

reaction to temperature changes, 135-142

Duplicity theory, 37-39, 73

Ellipsoid (lentiform body), 88, 89

of cones, $42,56,90$

of rods, $43,44,56,89$ 
Epithelial pigment layer (see pigment epithelial layer)

External limiting membrane, 18, 39, $50,51,153$

External scleral sulcus, 2

Eye, in relation to body size, 3 shapes of, in various vertebrates, 3 size of, in various vertebrates, 2,3

Fibers, centrifugal nerve, 26, 27

of Müller (Müllerian fibers), 26, 27, $50,51,53$

optic (see optic fibers)

retino-motor, 81

zonular, 13

Flicker, 38

Fovea centralis, 25, 29, 33, 35, 54, 71, 95-103, 108-119

concaviclivate, 104, 117, 119

convexiclivate, 104, 117, 118

differentiation of, 54

double, 98-102

nasal, 98-102

of various vertebrates, 96-103, 117 temporal, 98-103

Foveola, 29

Fundus specialization, in various vertebrates, $66,95-104$

Fuscin, 16, 86

Guanine, 6, 17

Internal limiting membrane, 18, 50, 51,118

Iodopsin (visual violet), 128, 143 absorption spectrum of, 130

Iris, 9, 11

color of, 11

muscles of, 9

reaction of, 91

retinal portion of, 11

variations of, 11

Kolmer's "droplets" (see "droplets" of Kolmer)

Lamina cribrosa, 4 vitrea, 16

Lateral (external) geniculate body, $19,115,116$

Layers of retina, 18 degeneration of, in vitamin A deficiency, 153-158 differentiation of, 48-51, 58, 59

responses of, to light, 84,85

width of, in relation to visual acuity, 104-107

Lens, origin of, 13

shapes of, 13, 14

suspensory ligament of, 7

variations of, $13,14,67-70$

Lentiform body (see ellipsoid)

Macula, 25, 29, 54, 101-103, 108-119

Melanin, 16, 17

Metathalamus, 19

Migration of pigment (see pigment migration)

Muscle, Brücke's, 8

ciliary, 8

dilatator pupillae, 9

Müller's, 8

sphincter pupillae, 9

"Myoepithelial" cells, 9

Myoid, of cones, 42, 47, 55, 77, 80 of rods, $43,47,79$

Neopallium, 115

Neuroepithelium, 48

Night blindness, 124, 130, 143-150

Oil globules (droplets), 42-44, 61, 90

Optic, cup, 16

decussation, 112-114

fibers, 109, 112, 113, 115

tracts, 108, 109

vesicles, 48

Ora serrata, 7

Orbiculus ciliaris (pars plana), 7

Paraboloid, of cones, 42, 56, 61, 90 of rods, 44, 56, 89

Pars ciliaris retinae, 8

Pars iridica retinae, 8

Pecten, 103, 104

Photomechanical responses of retina, 74-92

cone movements in, 77-79

in relation to central nervous system, 81,82

in relation to temperature, 80

in relation to various chemicals, 80 , 81

pigment migration in, 74-77, 80

rod movements in, 79

Photoreceptors (see cones and rods) 
Pigment epithelial layer, $6,7,16$, 71

cells of, 16

in relation to rhodopsin, $86,87,120$, 122,130

origin of, 48

pigment of, in various vertebrates, $16,17,62,67,71,74,90$

function of, 86-90, 92

Pigment migration, 74-92

in various vertebrates, $74-83$

Porphyropsin, 125-128

absorption spectrum of, 126

cycle, 127

occurrence of, 126-128

properties of, 126

Primary optic center, 19

Pupil, 9

reaction of, 91

slit (oval), 14, 15, 62, 65, 91, 92

variations of, 14

Refractive index, of retina, 118, 119 of vitreous, 118, 119

Retina, cells of (see cells)

degeneration of, 150-159

differential staining of, 46,84

differentiation of, 48-59

layers of, $18-20$

origin of, 16

pars optica retinae, $16-18$

photopigments of, $120-142$

pigment of (see pigment of pigment epithelial layer)

refractive index of, 118, 119

regeneration of, 157-159

resolving power of, 94

structure of, 16-37

thickness of, in various vertebrates, 19-24

Retinine (visual yellow), 122, 124$127,133-142$

in relation to "droplets" of Kolmer (see "droplets" of Kolmer)

in relation to vitamin $\mathrm{A}, 133-$ 135

Rhodopsin (visual purple), 59, 62, 86, $88,120-142$

absorption spectrum of, 120-122, $124,126,130$

cycle, $122-125$

in relation to "droplets" of Kolmer, 133, 134 in relation to epithelial pigment, 120,122

in relation to the visual cycle, $124-$ $130,133-142,151$

regeneration of, $86,87,121,122$

Rods, classification of, 45-47

conducting systems of, 30-33, 35

connections (endings) of, 25,45

degeneration of, in vitamin A deficiency, $150-158$

differentiation of, 50-59

double, 27

function of, 37-39, 120

green, $27,62,95$

in relation to visual acuity, 93-95

measurements of, $20,22,23,43$

nuclei of, 39,84

number of, 41

origin of, 49

photomechanical responses of, 47 , 88-90

photopigments of (see porphyropsin and rhodopsin)

red, 27,62

size of, $43,94,95$

staining reaction of, 46

structure of, 39, 43

thresholds of, 37,94

variations of, 43

Rosettes, 154-156

Sclera, of various vertebrates, 4,5

Suspensory ligament of lens, 7

Tapeta, 6, 7, 16, 17, 61, 65, 66, 71, 87,

89, 98 (see choroid, specialization of)

Theory, duplicity, of von Kries, 3739,73

of double retina of Parinaud, 37

of pigment migration, 86-92

of significance of the fovea (Walls), $116-119$

photochemical of Hecht, 122, 123

"transmutation" theory of Walls, 72,73

Threshold of visual cells, 37

Uvea (uveal tract), 6-12

Vision, binocular, 109, 110, 112-114

diurnal, 60-73

nocturnal, $60-73$

photopic, 35,37

scotopic, 35,37 
Visual acuity, 30,93, 94, 104-107, Visual yellow (see retinine)

111

Visual cells (see cones and rods)

Visual purple (see rhodopsin)

Visual thresholds, 143

Visual violet (see iodopsin)
Vitamin A, 122, 124-128, 130, 133$136,140-152,158,159$

deficiency, $130,132,133,142-159$

storage of, 149

therapy, 146-159 





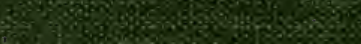

8 ing

9.8.

\%8.

2050\%

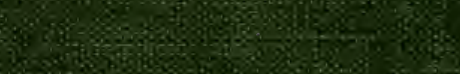

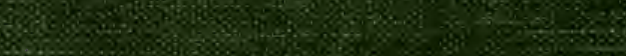

(4)

K.0.

(3)

(3)

acs.

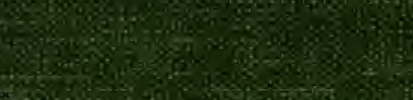

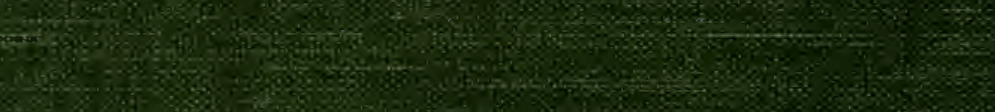

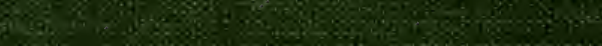

(4)

2.0.

(1)

and is

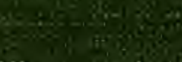

906 and

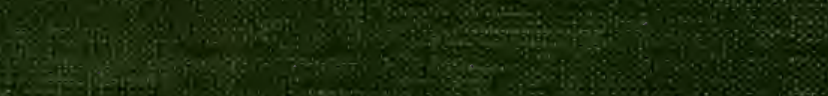

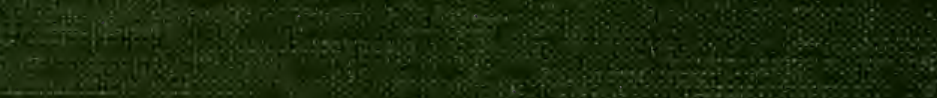

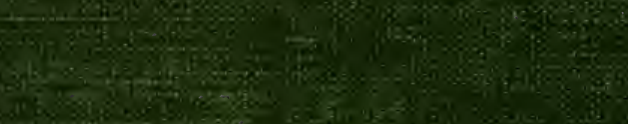

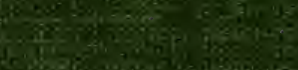

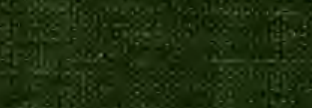

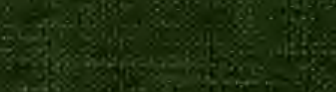

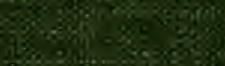

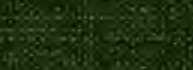

65

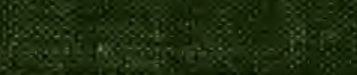

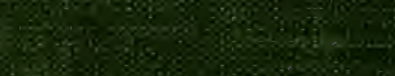

is 